Volume 3 Number 2

Supplement 3

November 2019

\title{
AJTES
}

\section{ALBANIAN JOURNAL OF TRAUMA AND EMERGENCY SURGERY}

\section{Focus on \\ "Trauma and Emergency Surgery" and not only...}

Official publication of

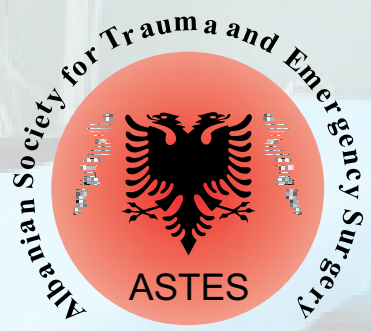


Volume 3 - Number 2 - Supplement 3

\section{Focus on \\ "Trauma and Emergency Surgery" and not only...}

official publication of

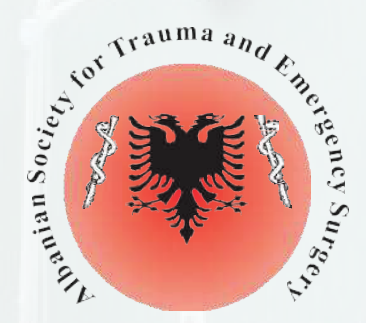

ISSN: 2521-8778 (print version)

ISSN: 2616-4922 (electronic version)

M \& B publications
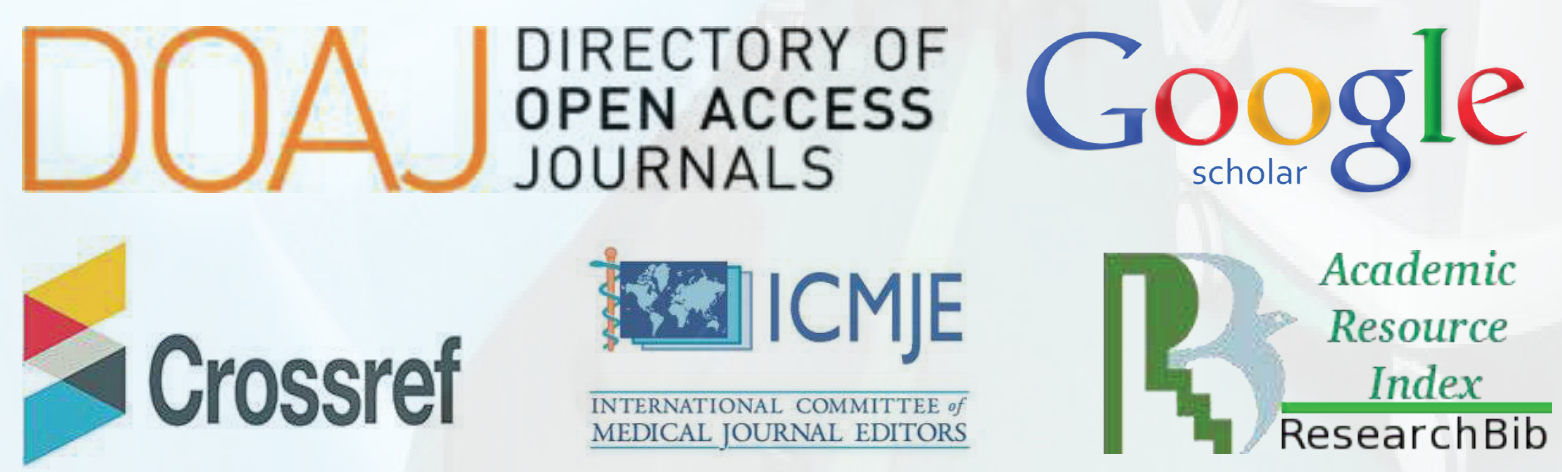


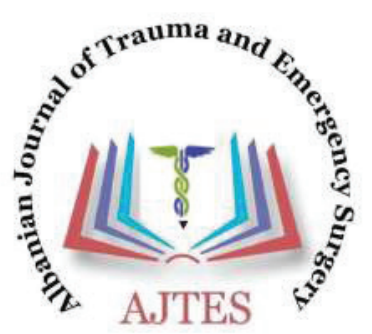

\section{Albanian Journal of Trauma and Emergency Surgery AJTES}

Official Publication of the Albanian Society for Trauma and Emergency Surgery - ASTES

Chairman of the editorial board:

Asc. Prof. Agron Dogjani MD, PhD, FACS FISS (ALBANIA)

\section{Editor in Chief}

Asc.Prof. Rustem CELAMI MD, PhD (Albania)

\section{Deputy Editors}

Prof. Lutfi ZYLBEARI MD, PhD (Macedonia)

Asc.Prof. Rudin Domi MD, PhD (Albania)

Edvin SELMANI MD, PhD (Albania)

Ilir ALIMEHMETI MD, PhD (Albania)

Asc.Prof. Majlinda NAÇO, MD, PhD. (Albania)

\section{National Editorial Board}

Prof. Arben GJATA MD, PhD

University of Medicine, Tirana, Albania

Prof. Xheladin DRAÇINI MD, PhD

University of Medicine, Tirana, Albania

Prof. Nikollaq KAÇANI MD, PhD

University of Medicine, Tirana, Albania

Prof. Etmont ÇELIKU MD, PhD

University of Medicine, Tirana, Albania

Prof. Arben BEQIRI MD, PhD

University of Medicine, Tirana, Albania

Prof. Ilia MAZNIKU MD, PhD

University "Alexander Xhuvani", Elbasan, Albania

Prof. Arvin DIBRA MD, PhD

University of Medicine, Tirana, Albania

Asc. Prof. Edmond NUELLARI MD, PhD

University of Medicine, Tirana, Albania

Asc.Prof. Sokol XHEPA MD, PhD

University of Medicine, Tirana, Albania

Asc.Prof. Aleksander HOXHA MD, PhD

University of Medicine, Tirana, Albania

Asc. Prof. Ridvan ALIMEHMETI MD, PhD

University of Medicine, Tirana, Albania

Prof. Pirro PRIFTI MD, PhD

University "Alexander Moisiu”, Durres, Albania

Asc. Prof. Edmond ZAIMI MD, PhD

University of Medicine, Tirana, Albania

Asc. Prof. Rudin DOMI MD, PhD

University of Medicine, Tirana, Albania

Asc. Prof. Besim BOCI MD, PhD

University of Medicine, Tirana, Albania Asc.Prof. Fadil GRADICA MD, PhD

University of Medicine, Tirana, Albania Artid LAME MD, PhD

University of Medicine, Tirana, Albania

Arsen Seferi MD, PhD

University of Medicine, Tirana, Albania

Sokol ISARAJ MD, PhD

University of Medicine, Tirana, Albania

Alfred IBRAHIMI MD, PhD

University of Medicine, Tirana, Albania

Marsida Krasniqi, MD, PhD

University of Medicine, Tirana, Albania

Dritan TODHE MD, PhD

University of Medicine, Tirana, Albania

Gëzim GALIQI MD, PhD

Shkodra Regional Hospital, Albania

Leart BERDICA MD, PhD

University of Medicine, Tirana, Albania

Myzafer KAÇI MD, PhD

University of Medicine, Tirana, Albania

Shkëlzen OSMANAJ MD, PhD

University Hospital of Trauma, Tirana, Albania

Zamir DEMIRAJ MD, PhD

University Hospital of Trauma, Tirana, Albania

Henri Kolani MD, PhD

Gjergj SEMINI MD, PhD

American Hospital Albania, Tirana, Albania 
Prof. Joakim JORGENSEN MD, PhD, FACS (Norway)

Prof. Selman URANUES MD, FACS (Austria)

Prof. Dietrich DOLL MD, PhD, FACS (Germany)

Prof. John E. FRANCIS MD, PhD, FACS (USA)

Prof. Thomas B. WHITTLE MD, PhD (USA)

Prof. Sadi BEXHETI MD, PhD (Macedonia)

Prof. Vilmos VECSEI, MD, PhD (Austria)

Asc. Prof. Pantelis VASSILIU MD, PhD, FACS (Greece)

Prof. Massimo SARTELI MD, PhD, FACS (Italy)

Prof. Carlos MESQUITA MD, PhD (Portugal)

Prof. Mauro ZAGO MD, FEBS, FACS (Italy)

Prof. Boris SAKAKUSHEV MD, PhD (Bulgaria)

Prof. Bellal A. JOSEPH MD, FACS (USA)

Prof. Claudio TAGLIA MD, PhD (Italy)

Asc. Prof. Basri LENJANI MD, PhD (Kosovo)

Prof. D'Archivio LAFRANCO MD, DDS (Italy)

Prof. Mehmet ERYILMAZ MD, MBAH (Turkey)

Prof. Kenan KARAVDIC MD, PhD (Bosnia Herzegovina)

Asc. Prof. Bogdan DIACONESKU MD, PhD (Romania)

Kastriot HAXHIREXHA MD, PhD (Macedonia)

Ilir HASANI MD, PhD (Macedonia)

Asc. Prof. Nehat BAFTIU MD, PhD (Kosovo)

Asc. Prof. Nuhi ARSLANI MD, PhD (Slovenia)

Asc. Prof. Basri LENJANI MD, PhD (Kosovo) 
Albanian Journal of Trauma and

Emergency Sur-gery is the official

publication of AlbanianSociety

for Trauma and Emergency Surgery - ASTES

\section{Structure and Format}

AJTES is an open access, peer reviewed periodical journal that includes editorials, re-views,original articles, case reports, short report,ideasand opinions, bookreviews, perspectives, sem-inars, symposium and mini- symposium, ethics andrights, health care policy and management, practiceguides. The structure of each edition of the publi-cation comprises section categories determined by Editor and reflects the views of the Editorial Board.

\section{Editorial Expertise}

Guidance from Editorial Board consists of leadingauthorities from a variety and respective fields ofmedical science. Peer review-accomplish by expertselected for their experience and knowledge of a specific topic.

\section{Important notice for Author}

All manuscript must be submitted to the EditorialOffice through electronic post; e-mail: contact@astes.org.al. Please check the last page of each edi-tion "Instruction for Author": The work shall not bepublished elsewhere in any language without the written consent of AJTES. The articles published inthis journal are protected by copyright, which covertranslation rights and the exclusive right to repro- duce and distribute all of the articles printed in thejournal. No material published in the journal may bestored on microfilm or videocassettes or in electron-ic databases and the like or reproduced photographically without the prior written permission of AJTES.

\section{Permissions}

For information on how to request permissions toreproduce articles/information's from this journal, please contact Editorial Office.

\section{Advertising policies}

The journal accepts displayed and classified adver-tising. Frequency discounts and special position areavailable. The journal reserves the right to reject anyadvertisement considered unsuitable according tothe set policies of the journal. The appearance of advertising or product information in the varioussections in the journal does not constitute an en-dorsement or approval by the journal and/or its pub-lisher of the quality or value of the said product or of claims made for it by its manufacturer.

\section{Disclaimer}

The information and opinions presented in theJournal reflects the views of the author and notof the Journal or its Editorial Board or the Pub-lisher.

Publication does not constitute endorsement bythe journal. 


\title{
Abstracts
}

\section{3rd Annual Albanian Congress of Trauma and Emergency}

\section{Surgery}

\section{ASTES}

\author{
November 8-9 \\ Tirana, Albania
}

\author{
Congress President: \\ Agron Dogjani \\ P resident of Honour: \\ Carlos Mesquita
}




\title{
Official Publication of the Albanian Society of Trauma and Emergency Surgery - ASTES
}

\author{
Aims and Scope \\ Our aim is to promote \\ interest, knowledge and \\ quality of care in \\ emergency and trauma \\ surgery. ASTES was \\ formed in 2017, it seeks to \\ promote best practice in the \\ provision of emergency and \\ trauma surgery and acute \\ care surgery, from pre- \\ hospital care through \\ diagnosis, intervention and \\ intensive care to \\ rehabilitation. This is \\ supported by Countrywide \\ \& International \\ collaboration, scientific \\ research, development and \\ delivery of training courses, \\ and the work of the \\ specialist sections \\ (Polytrauma, Visceral \& \\ Chest Trauma, Skeletal \\ Trauma \& Sports Medicine, \\ Neurosurgical, Anesthesia - \\ Reanimation, Acute Care \\ Surgery, ENT \& \\ Ophthalmology \& \\ Maxillofacial, Radiology, \\ Nurse service, Disaster \& \\ Military Surgery...etc.) \\ ASTES holds an annual \\ scientific meeting - the \\ Albanian Conference for \\ Trauma and Emergency \\ Surgery (ACTES) and \\ produces a bi-annual \\ journal - the Albanian \\ Journal for Trauma and \\ Emergency Surgery
}

\section{Contents:}

ORAL PRESENTATIONS

POSTER PRESENTATIONS

INDEX 


\section{ORAL PRESENTATIONS}

$O P$ - 001

\section{Direct Peritoneal Resuscitation in Open Abdomen Management}

\author{
Hayato KURIHARA
}

Authors Information:

Humanitas Research Hospital, Head, Emergency Surgery and Trauma SectionDepartment of General Surgery, Rozzano, Milan, ITALY

\begin{abstract}
Introduction:Damage control surgery is a staged approach to the trauma patient in extremis that improves survival, but leads to open abdominal wounds that are difficult to manage. We evaluated whether directed peritoneal resuscitation (DPR) when used as a resuscitation strategy in severely injured trauma patients with hemorrhagic shock requiring damage control surgery would affect the amount of and timing of resuscitation and/or show benefits in time to abdominal closure and reduction of intraabdominal complications.

Open a abdomen strategy is new standard of care for many acute surgical and traumatic abdominal injuries. One of the main challenges is related to delayed abdominal closure and fascial retraction. Direct peritoneal resuscitation is associated with improved outcomes in these patients.

The damage control strategy shortens the interval to definitive fascial closure without affecting overall resuscitation volumes. As a result, this mitigates intra-abdominal complications associated with open abdomen and damage control surgery and affords better patient outcomes.
\end{abstract}

$O P$ - 02

\section{Training in Trauma Surgery, Challenges and Impact on Survival}

\section{Carlos MESQUITA}

Author Information:

General and Emergency Surgery Departments Trauma Center, Coimbra Central and University Hospital, PORTUGAL

\section{Abstract}

This presentation reflects the author's involvement in internationally recognized education and training models, as ATLS, ETC and DSTC, and in the Portuguese and UEMS Boards of Emergency Surgery

Do different countries need very different things? I don't think so, for the simple reason that in Europe and about the challenges we face, with regard to trauma and other medical and surgical emergencies, similarities are much greater than differences.

- We all agree on the need for a trauma system "to assure that patients (...) seamlessly receive the proper care, in the proper locations, with proper interventions and, if necessary, transfer to a hospital able to provide the best and most appropriate care" (www.aast.org).

- We all agree that teamwork is necessary for prehospital care, transportation, emergency room care, intensive care, surgery and in/post-hospital rehabilitation.

- We all agree on the need for trauma registries with, as much as possible, global follow-up of patient's course.

- And finally, we all agree that it is necessary to educate, how to prevent and how to treat.

This is also apparent from the recommendations of the European Trauma Course Organization (ETCO) about equipment and facilities: complete trauma team, trauma admission bay close to the ambulance entrance, enough space and adequate lighting, adjacent operating room to allow emergency procedures, standard equipment for the initial management of major trauma, immediate availability of additional equipment as difficult airway equipment, X-ray, ultrasound machine, surgical instruments, readily available blood products and massive transfusion equipment, co-located CT scanner to allow immediate imaging and access to angiography and interventional radiology, 24 hours a day within 30-60 minutes of request.

$O P$ - 003

Preperitoneal Pelvic Packing in Pelvic Fractures: When and How? 


\section{Hayato KURIHARA}

Authors Information:

Humanitas Research Hospital, Head, Emergency Surgery and Trauma Section, Department of General Surgery, Rozzano, Milan, ITALY

\begin{abstract}
Introduction: Severe pelvic trauma is one of the most severe injury in trauma. A multidisciplinary approach and local algorithm should be mandatory in any institutions accepting injured patients. Preperitoneal pelvic packing is part of a "pelvic trauma protocol" and represent a good alternative or complementary adjunct to the bleeding control pathway. Patients with hemodynamic instability due to pelvic fracture-related bleeding can have lifethreatening hemorrhage. Management options for hemorrhage control in complex pelvic fractures include bony stabilization, angioembolization, and preperitoneal pelvic packing (PPP). The indications for PPP as well as the operative technique and outcomes will be described in this review. PPP directly addresses the bony and venous bleeding of complex pelvic fractures, which results in the majority of blood loss in exsanguinating patients. As such, PPP should be considered for pelvic fracture patients who remain hemodynamically unstable despite red cell transfusion.
\end{abstract}

Keywords: Hemodynamically unstable; Hemorrhage; Pelvic fracture; Pelvic packing.

$O P$ - 004

Present and Future of Emergency Surgery as Independent Specialty in Italy: Is the Rescue Surgery Turning the Underdog into A Hero?

\section{Antonio La GRECA}

Author Information:

Department of Emergency Surgery, Catholic University of the Sacred Heart, School of Medicine, Rome, ITALY

\footnotetext{
Abstract

Introduction: Early in the Eighties, Italy was a pioneer country in teaching emergency surgery as an independent specialty. At the time, a specific residency program was active,
}

but the new European residency rules in the Nineties compelled the University system to cancel it. As a consequence, the emergency surgeon has been traditionally considered as "a general surgeon somehow dealing with emergency" and therefore treating on a parttime basis, and without specific training, patients admitted to the Emergency Department for acute surgical illness or trauma, who not only deserve specific surgical attention under a technical point of view, but also show a peculiar physiopathology deserving an "intensive care” approach usually not fully addressed by a general surgeon.

Recently, specific attention has been given in Italy to an increasing peculiar workload for the emergency surgeon, especially after the new laws introducing the emergency and trauma network as a core part of the National Health Service. Thus, this specialty, based on the concept of Acute Care Surgery introduced in the USA with its five pillars (trauma, non traumatic emergency, intensive care, elective surgery, rescue surgery), is gaining a new recognized role and specific emergency and trauma wards are increasingly introduced at least in the most important Emergency and trauma centers.

Keywords:general surgeon, Emergency Department, emergency surgery, trauma

$O P$ - 005

An Organisation of Trauma Departments in Slovenia, A view from a Regional Hospital

Teodor PEVEC,Simona KALŠEK

Authors Information:

General Hospital Ptuj, SLOVENIA

\begin{abstract}
Public health service is organized in such a manner so as to ensure all the inhabitants of the Republic of Slovenia the emergency medical assistance available at all times, including emergency transport and provision of emergency medicinal products as soon as possible and as close to the date of their manufacture as possible and during transport. Emergency medical assistance means the implementation of necessary measures by
\end{abstract}


doctors and their teams with persons whose life is imminently threatened due to illness or injury and persons, respectively, who could be affected by such a risk in a short period of time according to the symptoms. Emergency calls are received and processed by the emergency medical assistance service which forms an integral part of the public health service network.

Slovenia, officially the Republic of Slovenia, is a sovereign state located in southern Central Europe at a crossroads of important European cultural and trade routes. It covers 20,273 square kilometers and has a population of 2.07 million.We can see a great progress in the care of trauma patients.A basic-measures with which we have made better results than in past are: better prehospital treatment, a network of hospitals, an ATLS concept, a score evaluation systems.Challenges for the futures are: dispatchers centers, even better prehospital treatment and better interhospital transmissions.

Keywords:Trauma, patients, prehospital treatment.

OP - 006

\section{Polytrauma Trauma score Challenges in Management}

Agron DOGJANI ${ }^{1}$, Hysni BENDO ${ }^{2}$, Amarildo BLLOSHMI ${ }^{2}$

Author Information:

${ }^{1}$ General Surgeon, University Hospital of Trauma, Tirana, ALBANIA

${ }^{2}$ General Physician, National Center of Medical Emergency, Tirana, ALBANIA.

\section{Abstract}

Introduction: Trauma is the leading cause of death for the population of 1-44 years old and occupies the third place after cancer and cardiovascular disease in all age groups. As a result of the improvement of the treatment strategy for traumatized patients, we have a reduction in post-traumatic morbidity and mortality from $40 \%$ in the 1970 s to $10 \%$ in the new century. These achievements consist mainly of improving trauma treatment standards by defining preand in-hospital treatment algorithms that are already applied in most countries.

Implementation of the concept for transport time of trauma patient which reduces
Implementation of the concept for transport time of trauma patient which reduces transport time and therapy during transport has made it possible to improve PT outcomes. Discussing the epidemiology and mechanism of injury in polytrauma and principles of evaluating policy at all levels; during transport, in the emergency department, in the intensive care unit, in the operating room, postoperative care until the patient leaves the hospital.

Identification of evaluation criteria for trauma patient such as: primary, secondary and tertiary assessment.

Examine the elements of hospital management related to accident time, mode of transport (ambulance, car accident, airway), transportation time etc and their impact on the performance of PT.

Emergency Assessment Techniques of trauma patient in Emergency department...

Keywords:Trauma, management, trauma assessment.

\section{ATLS ${ }^{\circledR}$ Course Albania Branch Step by Step}

Agron DOGJANI ${ }^{1}$, Arben GJATA ${ }^{2}$, Xheladin DRACINI ${ }^{2}$, Arvin DIBRA ${ }^{2}$, Kastriot

HAXHIREXHA $^{3}$; Alfred IBRAHIMI ${ }^{4}$, Fadil

GRADICA $^{5}$, Rustem CELAMI ${ }^{6}$, Skender BRATAJ

${ }^{7}$, Basri LENJANI ${ }^{8}$, Hysni BENDO ${ }^{9}$, Amarildo

BLLOSHMI ${ }^{9}$, Edvin SELMANI ${ }^{1}$, Erjona ZOGAJ ${ }^{10}$.

${ }^{1}$ General Surgeon, University Hospital of Trauma,

Tirana, ALBANIA.

${ }^{2}$ University of Medicine of Tirana; General

Surgeon, Tirana, ALBANIA.

${ }^{3}$ General Surgeon at Hospital of Tetovo, RN of

MACEDONIA.

${ }^{4}$ Anesthesia and ICU Service," Mother Theresa"

Tirana, ALBANIA.

${ }^{5}$ Lecturer at University of Medicine of Tirana;

Tirana, ALBANIA.

${ }^{6}$ Head of Medical Lab \& Imagery Department,

ALDENT University, Tirana, ALBANIA

${ }^{7}$ National Center of Medical Emergency, Tirana, ALBANIA.

${ }^{8}$ Emergency Clinic, University Clinical Center,

Pristina, KOSOVO

${ }^{9}$ General Physician, National Center of Medical

Emergency, Tirana, ALBANIA.

${ }^{10}$ Imagery Service University Hospital of Trauma,

Tirana, ALBANIA. 


\section{Abstract}

For more than 20 years, following the changes that took place in Albania in 1992, we receive information from trauma patients in the media about the delivery of trauma services. Police records are worthy of a war balance report. Trauma is estimated to be the cause of deaths in $13.7 \%$ / 100,000 people. Every two days three Albanian Citizens die because of Automobile Accidents... Changes have already taken place, but the question arises, who cares about the trauma and its management? Formerly and now the state ... ok ... but? Is this enough?

The Ministry of Health should have a "Trauma Committee" if yes... is it active?

Already of the three years at the heart of these medical emergency management networks is a National Center of Medical Emergency (NCME).

The trauma service delivery is now evolving along the lines of a central and spoken model with a concentration of expertise and specialization in the center surrounded by smaller units that feed from the center. The study showed a $19 \%$ increase in the chances of survival since the Introduction of these changes. Another 1,600 trauma victims are alive today due to developments in the administration of trauma patients in England over the past six years.

In this system or mode of organizing what is today the NCME or organizing the transport of traumatized from the scene to the University Hospital of Trauma... by medical staff who have done a substantial part of the PhTLS $^{\circledR}$ course ... How is educational status of personal in other regions far from Capital? ATLS $^{\circledR}$ student courses in our country have been implemented by few and individually... ASTES, by default rules, has acquired the right to organize ATLS ${ }^{\circledR}$ in Albania...

$O P$ - 008

\section{Acute Cholecystitis in Frail Patient}

\section{Hayato KURIHARA}

Authors Information:

Humanitas Research Hospital, Head, Emergency Surgery and Trauma SectionDepartment of General Surgery, Rozzano, Milan, ITALY

\section{Abstract}

Introduction: Acute cholecystitis (AC) is the most frequent complication of cholelithiasis and one of the most common conditions requiring emergency surgery in the elderly. Cholelithiasis accounts for $90 \%-95 \%$ of all causes of AC, while acalculous cholecystitis accounts for the remaining $5 \%-10$

The elderly is at high risk to present an episode of AC, and up to $6 \%$ of elderly patients will experience severe AC. Laparoscopic cholecystectomy (LC) is currently the gold standard for the management of acute calculous cholecystitis, with preference for early intervention. In the elderly, however, disease characteristics, comorbidities, and poor functional status augment the risks associated with surgical intervention, which may result in increased morbidity and mortality. Most literature consider as elderly patients those whose age is equal or greater than 65 or 75 years, though these thresholds may not be the most appropriate from the practical point of view. In the present aging society with frail patient's acute cholecystitis represents an everyday challenge in most surgical teams.

A correct stratification of frailty in the elderly patient is therefore mandatory. Multidisciplinary approach and alternative surgical and endoscopic techniques are helpful to obtain the best possible outcome for these population of patients.

Keywords: Acute calculous cholecystitis, Elderly, Frailty, High-risk patients, Diagnosis, Surgery, Antibiotics

OP - 009

Duodenal fistulas surgical strategies and the role of negative pressure wound therapy

\section{Antonio La GRECA}

Authors Information:

Assistant Prof and Senior Staff General, Emerg. Trauma, Rescue, Acute Care Surgery; Vascular Access, Advanced Ultrasound, Rome, ITALY 


\section{Abstract}

Introduction:Duodenal fistulas usually follow the breakdown of a previous suture. They have a deep anatomical location, consent the transit of enteric fluid rich in activated enzymes, have a very high output and present problems of skin care1. Mortality rates range from 7 to $40 \%$. Their treatment usually consists in multiple surgical interventions, often by means of damage control procedures due to local and systemic adverse conditions. Damage control means avoiding new sutures on the duodenum or any intestinal anastomosis and allowing duodenal output to exit the abdomen in an easy and direct way in order not

to contaminate the peritoneal cavity. Tubes inside or in the proximity of the duodenal hole usually serve this purpose.

Good drainage in complex, infected or also deep wounds can be achieved by negative pressure wound therapy (NPWT), removing from every site of the wound with the use of gauzes and/or open cell polyurethane foam and a suction device. Moreover, the sealed wound care improves infection control and patient management. Previous contraindications to NPWT in case of exposed bowel (such as in an enteroatmospheric fistula) have been replaced by an extensive peri-visceral use thanks to newly designed foams and suction techniques. However, specific descriptions of NPWT for the treatment of duodenal fistulas are not reported. We designed an effective NPWT application after reporting three clinical cases of successful duodenal breakdown fistula treatment in the context of rescue surgery. Alternative techniques, such as endoscopic drainage with special prosthetic and suction approaches, including endoluminal NPWT, might also be effective.

Keywords:Duodenal fistulas, Good drainage, Damage control

$O P-010$

Impact of Acute Appendicitis Surgical Options on Intensity of Pain at post-Op Care

SerhiiMITSUK $^{1}$, Ihor HAIDA ${ }^{1}$, Borys BILYK ${ }^{1}$

Authors Information:

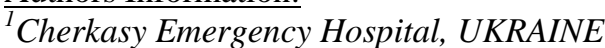

\section{Abstract}

Introduction: Acute appendicitis is a very common emergency surgery disease. All over the world as well as in our emergency hospital the question of surgical options remains.

The aim is to determine the dependence and intensity of post-op pain for acute appendix patient.

Materials\&Methods: The following criteria were used while assessing the pain; Patient's assessment of pain on a scale from 1 to 10 during post-op care prior to taking painkillers; The frequency of painkillers injection in 24 hours; Surgical options: appendectomy, laparoscopic appendectomy, appendectomy with drainage of the abdominal cavity, laparoscopic appendectomy with drainage of the abdominal cavity. The study has been undertaken by Surgery Department \#1, Cherkasy Emergency Hospital, Ukraine since March 2019 till August 2019... Results: We have 39 patients so far: 22 women and 17 men with acute appendicitis diagnosis (phlegmonous, catarrhalis, gangrenous) who required surgical treatment. The average age of women is 31.6 years (between 19 and 68 years). The average age of men is 29,3 years (between 18 and 48 years). The average days of receiving inpatient care-3.3 days....

Conclusions:Pain intensity and the frequency of painkillers injection for acute appendix patients depend on the surgical options. Laparoscopic appendectomy gives better result in pain reduction during post-op care in comparison to common appendectomy which causes considerable pain during post-op care. Drainage has almost impact on pain intensity Keywords:Pain Acute appendicitis, phlegmonous, catarrhalis, gangrenous...

$O P-011$

Synchronous Colorectal Cancer, a Concern of Diagnosis and Treatment, our Case Series

Faton T. HOXHA ${ }^{1,2}$, Rrustem MUSA ${ }^{1}$, Shemsedin HASHANI $^{1}$, Floren KAVAJA ${ }^{1}$, Arb F. HOXHA ${ }^{4}$, Ali DEVAJA ${ }^{1}$, Ferdinand KOMANI ${ }^{1}$, Shpresa HASIMJA $^{1}$, Arsim AJETI ${ }^{1}$, Fisnik KURSHUMLIU $^{3}$

Authors Information:

${ }^{1}$ University Clinical Hospital Service of Kosovo, Surgery Clinic, Pristina, KOSOVO; 
${ }^{2}$ University of Gjakova "Fehmi Agani”, Medical

Faculty, Gjakova, KOSOVO

${ }^{3}$ Institute of Anatomic Pathology, Medical

Faculty, University of Pristina, KOSOVO

${ }^{4}$ Trakya University, Medical Faculty,Edirne, TURKEY

\section{Abstract \\ Introduction: Synchronous colorectal} cancers, defined as two or more lesions in the same patient at the same time at diagnosis, are rare and deed a great challenge for treatment.

Materials and Methods: In five years, 375 patients underwent different colonic resections for colorectal cancer at the Department of General and Abdominal Surgery at the University Clinical Center of Kosovo. In the same group of surgically treated patients in elective end emergency, $1.07 \%, 2$ males and 2 females, were diagnosed intraoperatively with synchronous colorectal cancers (SCRCs). One of them previously treated for Ulcerative Colitis. Importance of preoperative diagnosis such as barium enema which may failed at the presence of annular carcinoma or inadequate bowel preparation and retained feces failure to inadequate bowel preparation and retained feces, or failure to perform completion colonoscopy at annular carcinoma, may be crucial intraoperatively to palpate the entire colon carefully before the end of the operation for CRC so that a misdiagnosis of SCRCs can be avoided. Full modern radiological investigations are precondition prior to the operation especially when the tumor is at an earlier histological stage. Failure to diagnose leads to errors in treatment and poor prognosis.

Conclusions: Palpation of the entire colon carefully before the end of the operation for CRC may reduce the risk of maltreatment of Synchronous colorectal cancers.

Keywords: Colorectal cancer, synchronous, surgery.

OP -012

Treatment of Patients with Symptomatic Cholelithiasis

Skender VELIU ${ }^{1}$, Jasmina KRÖPFL ${ }^{2}$, Dominika VRBNJAK ${ }^{3}$
Authors Information:

${ }^{I}$ Surgeon, Surgical ward, General Hospital $d r$. Jože Potrč Ptuj,SLOVENIA

${ }^{2}$ Nurse for controlling hospital infection, General Hospital Ptuj SLOVENIA

${ }^{3}$ Ass. Professor, University of Maribor Faculty of Health Sciences, Maribor, SLOVENIA

\section{Abstract}

In the recent years cholelithiasis has become one of the most common conditions affecting the digestive system worldwide. It affects approximately one tenth of the adult population, with the higher incidence in women. Symptoms are only present in 20$30 \%$ of the affected population. In the article we present the causes, symptoms, diagnostic tools available for screening as well as the current treatment guidelines for cholelithiasis. The reason for gallstone formation is in obesity, increased levels of cholesterol, increased age and certain medications especially oral contraceptives. The most common symptoms are pain in the right upper abdomen and in the epigastric area. The diagnosis is made based on the clinical presentation, laboratory findings and ultrasound of the abdomen. Current guidelines for conservative and surgical management of cholelithiasis are presented with the emphasis on laparoscopic cholecystectomy being the golden standard for the treatment cholelithiasis. Statistical figures and results of operative treatment of patients with cholelithiasis at General Hospital dr. Jože Potrč Ptuj, Slovenia for the period of 2013-2017 are presented as well.

Keywords:Cholelithiasis, conservative treatment, surgical treatment, laparoscopic cholecystectomy, guidelines.

$O P-013$

The Influence of Ursodeoxycholic Acid in Liver Functional Restoration in Patients with Obstructive Jaundice after Endoscopic Treatment 
Enver FEKAJ ${ }^{1 *}$, Preveza ABRASHI ${ }^{1}$, Vesel

SKENDERI $^{1}$, Afrim TAHIRI ${ }^{1}$, Besim

SERMAXHAJ1

Author Information:

${ }^{1}$ Department of abdominal surgery, University Clinical Centre of Kosovo,

Pristina, KOSOVO

\begin{abstract}
Introduction: The most common causes of obstructive jaundice are choledocholithiasis, strictures of the biliary tract, cholangiocarcinoma, carcinoma of pancreas, pancreatitis, parasites and primary sclerosing cholangitis.
\end{abstract}

Materials and Methods:In this prospective, randomized, open-labeled, and controlled study, 62 patients were enrolled. After diagnosis, eligible patients with obstructive jaundice who met inclusion criteria were randomly divided in the investigation group $(\mathrm{n}=31)$ in which has been administered UDCA, and in the control group $(\mathrm{n}=31)$. UDCA administration started twenty-four hours after endoscopic treatment.

Results:The difference of the average values of total and direct bilirubin, between the groups, was statistically significant at day 0 $(p<0.05)$, but at other evaluation days was not statistically significant, while the difference of the average values of ALT, AST, GGT, ALP, N/L ratio, urea, glucose, and creatinine, between the groups, was not statistically significant $(\mathrm{p}>0.05)$. The difference of the average values of albumin, between the groups, was statistically significant at the days 5,10 , and $15(\mathrm{p}<0.05)$.

Conclusions:UDCA has accelerated reducing the level of total bilirubin, direct bilirubin, GGT, and neutrophil/lymphocyte ratio, but did not decrease the level of ALT, AST, and alkaline phosphatase, and did not induce increasing of albumin level. UDCA had greater impact on GGT than in other functional liver parameters. The effect of UDCA did not depend on the gender and the age of patients, but did depend on the etiology of obstructive jaundice. It was more effective in patients with choledocholithiasis than in patients with malign stenosis of biliary tree.

Keywords:obstructive jaundice, ursodeoxycholic acid, treatment with UDCA
$O P-014$

The National Emergency System and its Extension throughout the Territory of the Republic of Albania.

\section{Skender BRATAJ}

Author Information:

Director of National Center for Emergency

Medicine, Tirana, ALBANIA.

\begin{abstract}
INTRODUCTION: Saving people's lives, guaranteeing their life quality, reducing patients' disabilities, alleviating the suffering of patients with chronic diseases and preventing morbidity and mortality is the goal of the National Emergency System. Our mission is accomplished through proper organization, discipline, ethical, deontological and professional working standards, standardized protocols, national and international guidelines, evidence-based medicine and continuous updating of contemporary scientific information. The once fragmented, chaotic, not organized prehospital emergency system was sporadic, uncoordinated, and the dispatcher who received the calls for medical help was the same who would go and give medical help. There were no control mechanisms, no protocols and no possibility of registering phone calls or patient medical records. Thereupon it was impossible to do scientific research, data collection, time to time evaluation in order to improve the service. Today we are talking about an integrated system, well organized and based on modern standards. How does this system really work? The Coordination Unit to the National Emergency Center is the brain, the command, and the control center. It is composed of several functional units or operating rooms that are: Evaluation, Management, Control, and Maxi Emergency Unit. In the Coordination Unit, each call for medical assistance is evaluated via telephone triage into the following steps: the location of the citizen at the time he/she is seeking help, the disease classification, the worsening code, and where the citizen is calling from. All this procedure is done electronically through an integrated program where every call is registered under the law, with the possibility
\end{abstract}


of its immediate hearing, archiving and collecting data and statistics for scientific Research.

Keywords:National Emergency System, 127, Emergency Care, National Centre for Emergency Medicine (NCEM).

$O P-015$

\section{Safety in Road Communication and Role of pre-Hospital Emergency Service}

Isuf BAJRAMI ${ }^{1}$, Bujar GASHI ${ }^{2}$, Avdyl PACOLLI $^{2}$, Haki DRAGUSHA ${ }^{2}$, Amire DRAGUSHA $^{2}$, Muhamet PETROVA ${ }^{2}$, Norik HOXHA $^{2}$

Authors Information:

Centre of Emergency Medicine, Pristina, KOSOVO

\begin{abstract}
Introduction:Around 1.25 million people die each year due to traffic accidents.

Traffic accident injuries are the leading cause of death in the most productive age group 20-45 years old. 20 to 50 million people end up with injueries, disability because of traffic accident injuries. Half of those who lose their life in road accidents are active participants in traffic - pedestrians, cyclists, bikers. If no preventive measures are taken, it is expected that in year 2030 traffic accidents will be the leading cause of death in general.
\end{abstract}

Material And Methods:data for this paper is extracted from QMU and archive protocols. I have cooperated with Emergency Centre of UCCK, and Kosovo Police - Pristina regional unit.Retrospective method of research and afterwards descriptive and analytical method has been applied.

RESULTS: According to Police statistics, the largest number of accidents in traffic in Kosovo have taken place in year 2013 - 5541 cases. The lowest number was registered in 2003 - 5541 cases. The largest number of accidents with casualties have taken place in year 2009 with 176 casualties, while 2016 had the lowest number - 110 casualties. The Centre of Urgent Medicine in Pristina had
1283 traffic accident interventions in 2918. The largest number was in August - 198 cases (15.4\%) while the lowest number was in May - 69 (5.4\%).

CONCLUSION: The main factor of traffic accidents remains human factor.

Traffic accidents increase the incidence of death and disability in general population.

Laws and their obedience, technical condition of vehicles, road infrastructure are also factors tha influence the number of traffic accidents.

Enforcing Emergent Medical Services, promoting and educating the population, institutional cooperatin, have positive impact in reducing the number of traffic accidnts, decreasing mortality and disability caused by traffic accidents.

Keywords: traffic accident, medical urgency, fatality, security.

$O P$ - 016

Telephone Basic Life Support (BLS-T).

\section{Skender BRATAJ}

Author Information:

Director of National Center for Emergency Medicine, Tirana, ALBANIA.

\begin{abstract}
Introduction:Saving a patient's life is the medical art as well as ancient and beautiful as difficult and that requires dedication. Saving people's lives and guaranteeing their life quality is the mission of the National Emergency System. Our mission is accomplished through proper organization, discipline, ethical, deontological and professional working standards, standardized protocols, national and international guidelines, evidence-based medicine and continuous updating of contemporary scientific information. The phone call toward 112/ 127 and the voice of the doctor or nurse that answers are the first hope of every citizen that his life is safe. Hello, emergency service, how can I help you? This is the first moment that the medical professional takes over your life!

This is where the protocol of telephone cardiopulmonary resuscitation, the battle
\end{abstract}


against time, the battle against the disease, the battle against death begins. The brain is the main organ that suffers from lack of oxygen. The brain damage begins after 6 minutes of cardiac arrest and after 10 minutes the damage becomes irreversible. This is why the immediate start of resuscitation maneuvers means buying time and stopping it at the moment these maneuvers are started. The lifesaving maneuvers will be continued by the medical team that arrives at the destination. After the CPR is adequately executed and the patient follows all the chain of survival, he will not only be saved but he can also have a normal life otherwise he will die or never wake up. The Telephone CPR protocol provides for a series of actions coordinated by emergency medical professionals....

Keywords:first aid, BLS-T, National Centre for Emergency Medicine (NCEM)

$O P-017$

\section{Bystander Cricothyroidotomy with} Household Devices

\section{Haqif GASHI}

Author Information:

Head of Anesthesia, Intensive Care and

Paintherapy RoMedKlinik Bad Aibling, GERMANY

\begin{abstract}
Introduction:In various motion pictures, medical TV shows and internet chatrooms, non-medical devices were presented as tools for life-saving cricothyroidotomies. However, there is uncertainty about whether it is possible for a bystander to perform a cricothyroidotomy and maintain gas exchange using improvised household items. This study examines the ability of bystanders to carry out an emergency cricothyroidotomy in fresh human cadavers using only a pocket knife and a ballpoint pen.
\end{abstract}

Materials and Methods: Two commonly available pens and five different pocket knives were used. Ten participants with no or only basic anatomical knowledge had to choose one of the pens and one of the knives and were asked to perform a cricothyroidotomy as quickly as possible after a short Introduction. Primary successful outcome was a correct placement of the pen barrel and was determined by the thoracic lifting in a mouth-to-pen resuscitation.

Results: Eight (80\%) participants performed a successful approach to the upper airway with a thoracic lifting at the end. Five participants performed a cricothyroidotomy and three performed an unintentional tracheotomy. Injuries to muscles and cartilage were common, but no major vascular damage was seen in the post-procedural autopsy. However, mean time in the successful group was $243 \mathrm{~s}$.

CONCLUSION: In this cadaveric model, bystanders with variable medical knowledge were able to establish an emergency cricothyroidotomy in $80 \%$ of the cases only using a pocketknife and a ballpoint pen. No major complications (particularly injuries of arterial blood vessels or the oesophagus) occurred. Although a pocket knife and ballpoint pen cricothyroidotomy seem a very extreme procedure for a bystander, the results of our study suggest that it is a feasible option in an extreme scenario. For a better outcome, the anatomical landmarks of the neck and the incision techniques should be taught in emergency courses.

Keyword:Bystander, Cricothyroidotomy, Anästhesie, Intensivmedizin, Schmerztherapie.

$O P-018$

Intraosseous Access as Part of Reanimation in Emergency Setting

Haqif GASHI

Author Information:

Head of Anesthesia, Intensive Care and Paintherapy RoMed Klinik Bad Aibling, GERMANY

\section{Abstract}

Introduction:Intraosseous access or Intraosseous infusion is a form of application in which infusions or medications are 
administered intraosseous (into the bone marrow cavity). For this purpose, a steel cannula is pierced into the bone, over which the substances can be administered due to the good blood circulation of the bone marrow cavity.

Intraosseous access is often used in child emergencies, as it is sometimes difficult to puncture the small veins with a vein cannula. In principle, however, it can be used at all ages and is increasingly used in adults. Intraosseous access is a comparable alternative to the venous catheter in the infusion of fluid, drug administration and blood collection. In an emergency, all common medications and blood products can be applied, and blood samples are also possible. Advantages of intraosseous puncture are the rapid application and high success rates $(>80 \%)$ with a low risk of complications. The disadvantages are the higher cost of material and a limited flow rate of puncture needles in some systems.

$$
O P-019
$$

Health Care Centers During Tourist Season, Challenges and Innovations in Managing Medical Emergencies

\section{Skender BRATAJ}

Author Information:

Director of National Center for Emergency Medicine, Tirana, ALBANIA.

\footnotetext{
Abstract

Introduction:Albania is a coastal country with a large number of beautiful beaches, frequented by many people. In the summer season in Albanian territory there is an increase in the number of local and foreign visitors. During this season many citizens move from urban areas to coastal towns and beaches, which are often far from major cities and away from hospitals. 4 years ago, was made evident that with the permanent organization of the health system, the time of arrival in case of medical emergencies and accidents in the coastal areas, was very long and the risk to citizens' lives was considerable. That is why the Ministry of Health and Social Welfare and the National Emergency Center decided to plan and
}

organize a prehospital system, specifically tourist health centers, that would function only during the summer season. The planning and organization processes were based on the territory, the number of visitors, the distance from regional and municipal hospitals and the infrastructure. In 2015, 22 tourist health centers were opened, which followed 25 in 2016, 26 in 2017-2018 and reaching 28 in 2019. All health centers were provided with a telephone number available to the Coordination Unit for medical coordination. The National Emergency Center, in order to further improve the medical service in these areas, but mostly to shorten the time of arrival to the patient decided to place an ambulance near these centers, so it was possible to cover the medical needs in all tourist areas. In 2015 there were 19,000 visits, in 2016 the number of visits increased by almost 37\% (26,000 visits), in 2017 the number of visits almost doubled (about 40,000). In the last two years, this number has grown at a very large percentage leading to 49,921 visits in 2018 and a record of 69,850 visits in 2019.The new organization of this service puts us in front of a new challenge to meet the increasing demands from year to year. Planning means preventing, so it's good that new challenges come with an already proven to be successful service.

Keywords:tourist care, seasonal health centers, national emergency medical centre

$O P-020$

Helicopter, Excellence Service of the National Center for Emergency Medicine.

Skender BRATAJ, Erald XHELILI

Author Information:

Director of National Center for Emergency Medicine, Tirana, ALBANIA.

\begin{abstract}
Introduction:Helicopter medical emergency service is the best option to cope with emergency medical emergencies and in areas without infrastructure. Modernization and completion, not only because of modern medical equipment and devices, but more because of legal adaptation and new mentality, where every Albanian or foreign patient in the territory of the Republic of
\end{abstract}


Albania is guaranteed life and treated with the same devotion and professionalism.

This service is already equipped with onboard medical equipment that is needed tomonitor, defibrillate, immobilize and treat patients at the scene, during transport, to more specialized health centers. The novelty of this topic is about introducing rigorously prepared medical staff to deal with the variety of problems encountered during lifesaving operations.

The purpose of this study is to reflect and analyze the work done so far by the NCEM regarding the service, the time of arrival, the difficulties and the problems encountered during the work.

Accurate data analysis, correct documentation of events and missions through the patient's file, receiving pre-departure information and completing the territorial card are essential for assessing the quality of the service, improving it, and reducing the disability of the patient. The Coordination Unit as an Innovation has every important role in conducting this service.

As a Conclusion: Emergency helicopter service for the natural, structural conditions of our country is indispensable for lifesaving, service improvement, and reduction of disability.

The current state of affairs aims to improve this service to be as quality in line with the standards of international scientific associations.

Keywords:helicopter; patient's file, followup

$O P-021$

\section{Prehospital trauma and the National Emergency Center.}

Skender BRATAJ, Indrit JAUPAJ

Author Information:

Director of National Center for Emergency Medicine, Tirana, ALBANIA.

\footnotetext{
Abstract

Introduction:Prehospital Trauma Life Support (PHTLS) is the global gold standard in prehospital trauma education and is taught in 64 countries. PHTLS is appropriate for EMTs, paramedics, nurses, physician assistants, physicians, and other prehospital providers. It is recognized around the world as the leading continuing education program for
}

prehospital emergency trauma care. The mission of PHTLS is to promote excellence in trauma patient management by all providers involved in the delivery of prehospital care. PHTLS is developed by NAEMT in cooperation with the American College of Surgeons' Committee on Trauma. The Committee provides the medical direction and content oversight for the PHTLS program. NAEMT's international education activities began in 1991 with the establishment of an NAEMT training center in Mexico to conduct PHTLS. Today, NAEMT education courses are taught in many countries worldwide. National Centre for Emergency Medicine has already conducted the second PHTS course in Albania. The first Albanian instructors are already certified and will serve as a faculty that will continue to train the personal that works within the emergency system in Albania. This presentation describes the history of PHTS promulgation in Albania, its mission and the role of NCEM in continuing education for emergency medical staff in Albania.

Keywords:PHTLS, NCEM, NAEMT

$O P-022$

The Role of Neurolysis in the Surgery of Traumatic Lesions of Peripheral Nerve.

Ridvan ALIMEHMETI ${ }^{1}$, Florian DASHI ${ }^{1}$, Gramoz BRACE $^{1}$, Ermira PAJAJ ${ }^{1}$, Arba CECIA $^{2}$, Thoma KALEFI $^{1}$,Jetmira KERXHALLIU $^{1}$, Roland PERGJETANI ${ }^{1}$, Arsen SEFERI ${ }^{1}$

Authors Information:

${ }^{I}$ Neuroscience Department, University of Medicine of Tirana, Neurosurgery Sevirce, University Hospital center "Mother Theresa" Tirana, ALBANIA

${ }^{2}$ Hunterian Neurosurgical Laboratory, Department of Neurosurgery, Johns Hopkins School of Medicine, Baltmore, MD, USA.

\begin{abstract}
Introduction:Neurolysis is the operative technique of cessation of adhesions and removal of fibrosis following traumatic damage to peripheral nerves in the event of preservation of anatomical continuity of the nerve fascicles. This technique, defined by Milles in the 1980s and revised over the years, remains a successful technique in the treatment of persistent traumatic neuroma.
\end{abstract}


But the extent of neurolysis remains a subject of discussion on a macro and microscopic level in peripheral nerve surgeons around the world.

Materials and Methods:Neurolysis related updated world literature is reviewed. Cases operated at the QSUT Neurosurgery Service with neurolysis for various traumatic peripheral nerve injuries have been studied. Long-term treatment outcomes are reviewed to quantify the rate of return to neural function after treatment.

Results:Several types of peripheral nerve traumatic mechanisms have been operated with the technique of "neurolysis" contusion, shock wave firing, jatrogenic intra-fascicular nerve injection, birth plexigraft injury, etc.

The type of repair ranges from aderenciolize, longitudinal epineurotomy, interphasicular neurolysis.

The degree of improvement depends largely on the degree of fibrotic fascicular injury, the length of the injured nerve segment, the distance from the injury to the affected muscles, the time from traumatic injury to surgical treatment, the neurolytic technique used, the competence of performing microscopic and knowledge of peripheral nerve pathology.

Conclusions: In the many years' experience of repairing peripheral nerve traumatic injuries at the QSUT Neurosurgery Service, it turns out that neurolysis remains a technique used successfully in selected cases. It has been applied alone or in combination with direct sutures or transplants of interrupted nerve segments contained in the same traumatized peripheral nerve unit.

The use of intraoperative neuro-physiological monitoring, in particular NAP (Nerve Action Potential), adds to the assurance of obtaining valuable fascicles at the end of the applied neurolysis.

Keywords: Neurolysis, traumatic, nerve.

$O P-023$

Spontaneous Intracerebral Hematoma; Is it Only Blood?

\section{Artur XHUMARI}

Author Information:

Neurosurgeon in the University Hospital Center

"Mother Theresa" Tirana, ALBANIA

\section{Abstract}

When a spontaneous intracerebral hematoma is disclosed in a CT scan, there are many different pathologies to be differentiated, further exams to be ordered and treatment options to be taken into consideration.

Through some latest operated cases we highlight the logical steps to undertake and discuss the pearls and pitfalls of managing intracerebral hematomas. Metastasis, cerebral aneurysm, hypertensive hematoma, GBM are some of etiologies we will discuss.

When taken into consideration the age, comorbidities, antecedents, anamnestic elements, then the imaging suggests the next steps to take for the etiological diagnosis as well as surgical or conservative management.

Keywords:Hematoma, intracerebral, spontaneous.

$O P-024$

\section{Cranial Emergencies in Neurosurgery}

\section{Baris SAYGILI}

Author Information:

Faculty of Medicine, Ankara, TURKEY

Former Neurosurgeon at American Hospital, Tirana, ALBANIA

\begin{abstract}
Emergency in Neurosurgery is complicated and totally different topic in Neurosurgery. The emergency cases can be caused generally after trauma and accidents but although some sporadic/spontaneous situations can cause urgent cases in Neurosurgery. Mostly fractures and haemoragies are the common emergency cases in neurosurgical practice due to trauma and accidents. But also, sometimes sporadic/spontaneous cases like SAH,cerebral abscess or hydrocephalus cases can cause the emergency pattern. We want to remind the general emergency topics in neurosurgery and share some case experiences that we saw and operated by us.
\end{abstract}


$O P-025$

\section{Delayed Posttraumatic Siringomyelia}

Ridvan ALIMEHMETI ${ }^{1}$, Florian DASHI ${ }^{1}$, Gramoz BRACE $^{1}$, Ermira PAJAJ ${ }^{1}$, Arba CECIA ${ }^{2}$, Thoma KALEFI ${ }^{1}$, Jetmira KERXHALLIU ${ }^{1}$, Roland PERGJETANI ${ }^{1}$, Arsen SEFERI ${ }^{1}$

Authors Information:

${ }^{1}$ Neursocience Department, University of Medicine of Tirana, Neurosurgery Sevirce, University Hospital center "Mother Theresa" Tirana, ALBANIA

${ }^{2}$ Hunterian Neurosurgical Laboratory, Department of Neurosurgery, Johns Hopkins School of Medicine, Baltmore, MD, USA.

\begin{abstract}
Introduction:Post-traumatic spinal syringomyelia is rarely found in the literature. In cases of thoracic vertebral lesions without medullary lesions, kyphotic stenosis with duct stenosis and subsequent arachnoid adhesions are thought to favor the creation of conditions for the formation of sirings in the proximal part of the spinal medulla from the level of the vitreous lesion.
\end{abstract}

Materials and Methods:Retrospective study of a case presented at the QSUT Neurosurgery Service for post-traumatic syringe 16 years after traumatic L1 fracture.

A 41-year-old car accident victim in 2001 undergoing compression fractures of the L1 body presented with retinal urinary and anal sphincter deficits, but without inferior motorsensory deficits. He underwent neurosurgical intervention to decompress the canalicular contents with laminectomy without column fixation at the fracture level.

In February 2018 he was experiencing pain at the fracture / decompression level with right inferior paresthesia paresthesia and gradual progression of distal motor impotence with gradual proximal progression.

In November 2018, as a result of the above neurological deficits listed, thoracic-lumbar MRI was advised to document the presence of sirings over the medullary lesion extending from L1 to T10, with swelling of the liquor content. Altered PESS.

Results: He underwent microsurgical surgery of gastric syringes with posterolateral medial apertures of the T12 level, emptying the cystic contents into the perineal spinal space and communicating these two spaces to avoid
recurrence.A MRI check in May 2019 documented a reduction in the use of sirings in support of improved symptom stability.

CONCLUSIONS: Consistent fracture deformities especially at the thoracic-lumbar passage level treated only with decompressive laminectomy, without instrumental fixation have a high probability of peer perfusion in duct stenosis.

Posttraumatic post-traumatic syringomyelia extending above the level of the medullary lesion is a rare consequence but should be suspected whenever there is a manifestation of neuraxial irritation (pain, paresthesia) or new motor-sensitive neurological deficits or previous sphincteric deficits.

Stirring of the syringes with the consequent reduction of the pressure and volume of liquor contained therein is possible by microsurgical operating methods thereby resolving medullary dysfunction.

Keywords: Syringomyelia, delayed, posttraumatic, fracture.

OP-026

Surgical treatment of temporal lobe epilepsy. Our experience with 40 patients.

\section{Arsen SEFERI}

Authors Information:

${ }^{1}$ Psychiatry Service at University Hospital Center

"Mother Theresa", Tirana, ALBANIA

${ }^{2}$ Sugery Department at University Hospital Center

"Mother Theresa", Tirana, ALBANIA

\begin{abstract}
Patient E. H., age 39, presents to Surgical Emergency, with a cut wound: Sinister mammectomy. According to family and patient referrals, the action came as a result of a suicidal attempt. Suffers from Epilepsy since the age of 2 and several years from depression, leading to a suicidal attempt before. She has been treated with antiepileptic drugs for years and underwent neurosurgical intervention in Italy a month ago, which was successful.

The patient shows a regular appearance and according to the chronological age, maintains partial eye contact. Her mimic expresses an apathetic and cold state, without alterations of her mood. Refers that after neurosurgical intervention she has had severe headaches for
\end{abstract}


weeks, pains that did not enable her to sleep or perform normal daily activities. In one of these crises the patient has injured herself, by removing her left breast. Communication is apathetic, cold, distant. Thoughts are negative and worrying about her health. Perception of the environment and reality, cognition and insight damaged. When asked why she chose to damage her breast, refers she had a feeling of emptiness and therefore wanted to remove it. Her headaches were also unbearable and this led her to perform this injury. Through communication the patient provides a lot of information about psychotic elements responsible for the bizarre action. Treatment with antipsychotics, antidepressants, mood stabilizers and anxiolytics, brought an improvement in the psycho-emotional state. Such cases are rarely described in contemporary literature, the consequences of severe psychotic and affective disorders.

$O P-027$

\section{Spinal Emergencies in Neurosurgery}

\section{Baris SAYGILI}

Author Information:

Faculty of Medicine, Ankara, TURKEY

Former Neurosurgeon at American Hospital, Tirana, ALBANIA

\footnotetext{
Abstract

Emergency in Neurosurgery is complicated and totally different topic in Neurosurgery. The emergency cases can be caused generally after trauma and accidents but although some spontaneous or sporadic situations can cause urgent cases in Neurosurgery.

Mostly fractures and spinal cord injuries are the common spinal emergency cases in neurosurgical practice due to trauma and accidents. But also sometimes sporadic /spontaneouscases like acute disc herniations that can cause cauda equina or conus medullaris syndrome,vertebral infectious abscess or vascular pathologies that causes spinal haemorrhage cases cancause the emergency pattern.

We want to remind the general spinal emergency topics in neurosurgery and share some
}

case experiences that we saw and operated by us.

$O P-028$

\section{Balloon Kyphoplasty for Traumatic and Osteoporotic Compression Vertebral Fractures, our Experience}

\author{
Artid LAME ${ }^{1}$, ErionSPAHO ${ }^{2}$, Gledion \\ FEJZO $^{2}$
}

Author Information:

${ }^{1}$ Department of Neurosciences, University of Medicine, Neurosurgery Service, University Hospital Centre "Mather Theresa" Tirana, ALBANIA

${ }^{2}$ Neurosurgery Service, University Hospital of Trauma, Tirana, ALBANIA
Abstract
Introduction: We present a review of patients diagnosed and treated for osteoporotic thoracic-lumbar compression fractures.
Materials and Methods:There were three patients diagnosed and treated for osteoporotic thoracic-lumbar compression fractures during the year 2018 in a private hospital. The patients' clinical data, imaging studies and operative procedures were reviewed retrospectively.
Results:There were three female patients ranging from 34 to 63 years old, (mean age 51.6 years old) identified. All three patients were diagnosed previously for osteoporosis. Time from symptoms onset to diagnosis and treatment varies from one month to three years.
Discussion:Vertebral osteoporotic compression fractures are almost twice as common as other fractures typically linked to osteoporosis, such as broken hips and wrists. They are located more in the lumbar and thoracic region.
Conclusion:Treatment with balloon kyphoplasty remains the gold standard in the treatment of Vertebral osteoporotic compression fractures with early significant improvement of symptoms.
Keywords: osteoporotic fractures, compression fractures, balloon kyphoplasty. 
$O P-029$

\section{Neurotizations in the $C_{5} C_{6}$ Proximal Avulsions of the Brachial Plexus}

Ridvan ALIMEHMETI ${ }^{1}$, Florian DASHI ${ }^{1}$, Gramoz BRACE $^{1}$, Ermira PAJAJ ${ }^{1}$, Arba CECIA $^{2}$, Thoma KALEFI ${ }^{1}$, Jetmira KERXHALLIU $^{1}$, Roland PERGJETANI ${ }^{1}$, Arsen SEFERI ${ }^{1}$

Authors Information:

${ }^{1}$ Neurscience Department, University of Medicine of Tirana, Neurosurgery Sevirce, University Hospital center "Mother Theresa" Tirana, ALBANIA.

${ }^{2}$ Hunterian Neurosurgical Laboratory, Department of Neurosurgery, Johns Hopkins School of Medicine, Baltmore, MD, USA.

\begin{abstract}
Introduction:In the 90s it was widely accepted that C5 and C6 were more widely used in neurotransmission techniques than $90 \%$ in neurotransmitters in $90 \%$ of nerve function regeneration, thereby compromising extracorporeal function.
\end{abstract}

At the QSUT Neurosurgery Service, these techniques have been successfully tested in follow-up for over 15 years, intensifying and updating with intraoperative monitoring techniques.

Materials and Methods: In all operated diagnostic cases of C5 and C6 avulsion supported by cervical MRI and ENMG, SSEP preoperatively. Neuro-technical techniques for C6 and C6 avulsion are applied:

1. with terminal nerve accessor spinal donor for resuscitation of the nervesupra-scapular as recipient.

2. With a $\mathrm{n}$. radial donor, the branch for the medial head of the muscle tricepsbrachi for resuscitation of the nerve-axillary recipient.

3. With a n. ulnar donor, a muscle-carpiulnaris (and median) trunk, as a donor for the resuscitation of the musculoskeletal nerve, a branch for musculoskeletal (brachialis).

Operational outcomes are reviewed and reviewed for trauma to surgical resection (3 months - 3.5 years).

Results: In all modes of operation, slight (M2), medium (M3) and satisfactory (M4) improvements in engine function were observed.The application of intraoperative neurophysiology to the microsurgical technique for neurorrhaphies with 10/0 nylon is fundamental in obtaining a functional result. Recently from trauma to operation, there was no effector morbidity in outcome and neuroticization.

Conclusions: Preoperative study of the viability of paralyzed muscles most important for predicting the surgical outcome of neurotization.The use of techno-vestiges in the neurotization of the anterior (middle and anterior) muscles as well as the berry flexors (biceps and brachialis) provide a more secure functional recovery than the technicoseconds. Microscopy of intraoperatories is indispensable in the application of neurotization.Post-operative rehabilitation with electrovascular stimulation andmuscular bone factor Important in obtaining maxillofacial efficacy of plexigs.

Keywords:Neurotizations, paralyzed muscles, surgery.

$O P-030$

Postoperative Shock; How to Anticipate and Manage it Conveniently?

Mauro ZAGO,

Authors Information:

General Surgery, Minimally Invasive Surgery Unit, Head, Policlinico San Pietro BOLOGNA - ITALY

\footnotetext{
Abstract

Introduction:Postoperative shock is a not so uncommon event and recognize many etiologies, ranging from intracavitary/intrabdominal hemorrhage, pulmonary embolism, septic shock due to surgical and not surgical complication, heart failure.

It could happen both after elective and emergency procedures.

Management of a patient with clear or impending postoperative shock requires a high index of suspicion of the surgical team and of the nursing team too. Ideally, a planning of the actions for a safe and effective monitoring should be established in any surgical ward, possibly with the help of
} 
telemonitoring systems even in non-highdependent unit.

The use of check-lists and a wide application of tools like the NEWS score are one of the most easily way to implement the culture of standardized monitoring of critical or potentially critically ill patient in the postoperative period. It is clear that the goal is to anticipate the need for rescue surgery, and/or put a patient requiring interventional or surgical repair of a postoperative complication in the best situation for recovering, decreasing the rate of failure to rescue.

In the era of acute care surgery, surgeons dealing with acute patients (all complicated patients after elective and emergency surgery are acute patients) have to know the surgical procedure undertaken and to stay close to physiology, which remains the mainstone for any decision.

Basic knowledges for a physiological assessment are mandatory, goal directed fluid management has a pivotal role too. Nowadays, some critical applications of Ultrasound should enter in the armamentarium of surgeons too. In particular, free fluid detection (E-FAST), basic lung US semeiology, US evaluation of volume replacement and heart function "at a glance".

It is time to improve skills and organizations for getting better outcomes in critically ill patients.

Keywords: fluid resuscitation, mean arterial pressure, operation, surgery,

$O P-031$

\section{Acute Airway-Threatening Presentations of Thyroid Diseases in Very Old Patients}

\section{Carlos MESQUITA}

Author Information:

General and Emergency Surgery Departments, Trauma CenterCoimbra Central and University Hospital, PORTUGAL

\footnotetext{
Abstract

Acute airway-threatening from thyroid diseases is not common but may occur due to
}

giant goiters, hematomas and thyroid malignancies, among others. The author underline the need for the surgeon working in acute care setting to be familiar with thyroid surgery.

Three recent cases, in very old patients, of acute airway-threatening from thyroid diseases are described:

1. A 91-year-old male with known giant right-sided thyroid mass, with a cytologic study suggestive of follicular tumor, who had refused thyroid surgery two months earlier, was hospitalized for acute exacerbation of dyspnea and hoarseness. Cervical mass extended from the hyoid bone to the upper mediastinum, with left shift of cervical structures. Right thyroidectomy and istmectomy were urgently performed.

2. A 88-yeard-old female with a known bilateral large cervical mass was admitted due to stridor. Computed tomography revealed a large retrosternal compressive goiter with a remaining tracheal lumen of $2 \mathrm{~mm}$. Surgical risk was significantly aggravated by a severe aortic stenosis. Since there was no place for a classic tracheostomy and endotracheal intubation could be difficult or impossible, everything was prepared in order to anesthetize the patient under ECMO, which, however, did not become necessary. Intubated with the aid of a fiberscope, a total thyroidectomy was possible, after partial sternotomy.

3. A 78-year-old female complaining of stridor and dysphagia, from a large right cervical mass, with a $4 \mathrm{~mm}$ tracheal lumen was admitted. A tracheostomy was performed but, due to airway obstruction not completely resolved by this procedure, a right lobectomy with isthmectomy was performed urgently. Although cytologic study was suggestive of papillary carcinoma, the degree of local infiltration, evident at the time of surgery, first drew attention to the possibility of an anaplastic thyroid cancer.

Acute airway-threatening from thyroid diseases is not common. Early recognition of these conditions and urgent, partial or total thyroidectomy may be necessary to alleviate symptoms, when tracheostomy is not an 
effective option. The surgeon working in an acute care environment should be aware of these possible scenarios and be prepared to solve them.

$O P-032$

Esophageal Trauma from Foreign Bodies. Literature Review and our Experience.

Arvin DIBRA, Etmont ÇELIKU, Inid JORGONI, Arvit LLAZANI, Drini SHEHI.

Authors Information:

${ }^{1}$ Surgery Department, University Hospital Center "Mother Teresa", Tirana, ALBANIA

\begin{abstract}
Introduction:Esophageal trauma from foreign bodies, it's not a rare condition in our polyvalent emergency. The esophagus is the most common site of foreign body impaction. Food impactions cause most esophageal foreign bodies. Large, smooth food pieces (e.g. steak, hot dogs) are particularly easy to swallow inadvertently before being chewed sufficiently. Bones, particularly fish bones, may be swallowed if the meat in which they are embedded is not chewed sufficiently. It can be complicated with esophageal perforation, so all team in operating room must be prepared for this condition, especially the surgeons.
\end{abstract}

The foreign body, which may be food or dental prosthesis, may cause spontaneous esophageal injury or injury during attempts to remove it. The primary choice to solve this situation is endoscopic management not only to diagnose but also to treat the complication. Emergent endoscopy is required for sharppointed objects, disk or button batteries and any obstruction causing significant symptoms. Esophageal perforation has a high mortality and morbidity and all the procedures to remove the foreign body increase the risk for it. We are bringing you our revision and experience.

Keywords:Esophageal trauma, foreign bodies, emergency, fish bones.

$O P-033$

Diagnostic and Therapeutic Laparoscopy in Abdominal Trauma
Simona KALŠEK, Jožeta POTRČA

Authors Information:

${ }^{1}$ Department of Surgery, General Hospital,Ptuj, SLOVENIA

\begin{abstract}
Introduction: The aim of present study is to evaluate the role of laparoscopy in blunt and penetraiting abdominal trauma.

Materials and Methods: We performed a review of the literature using Pub Med database. The key words were laparoscopy and abdominal trauma. In last 10 years there were 785 articles published.
\end{abstract}

Results: Laparoscopy is most useful in hemodinamic stable patients with penetraiting abdominal trauma. The sensitivity and specificity of diagnostic laparoscopy is $99 \%$ i.e. $100 \%$. In $92 \%$ therapeutic laparoscopy was successful.

In blunt abdominal trauma the role of laparoscopy is less clear. It is an option in selected patients who are hemodinamic stable and have a fluid collection of unknown origin on CT scans. In selected cases it is also optional in patients with bowel perforation, liver and splen trauma.

Conclusion: Laparoscopy is a useful diagnostic and therapeutic procedure in stable patiens with abdominal penetraiting wound. In blunt abdominal trauma it's role is more limited.

Keywords: laparoscopy, abdominal trauma.

OP -34

Management of Liver Trauma

\section{Skender BUCI, Dorina SHTJEFNI}

Authors Information:

Surgery Service, University Hospital of trauma, Tirana, ALBANIA

\begin{abstract}
Introduction:The hospital Trauma Centers could be able to management severe liver and biliary tract injuried but should have the following criteria or pre-condition like; theTeam of surgeons trained for this purpose, ultrasound installed in the operating room, liver injury assessment with angio-CT, 24hour operating room equipped, resuscitation, options for arterial embolization.
\end{abstract}


Liver injuries have been found in $20 \%$ of cases of polytrauma, according to most reports from various trauma centers. Car accidents are the leading cause of Blunt Liver injuries.

Diagnosis of Liver injury has undergone a significant evolution in recent decades, due to ongoing developments in the field of imaging.

The diagnostic and therapeutic strategy is dependent on the hemodynamic status of the traumatized patient.

Currently, non-operativ management is preferred in more than $80 \%$ of cases, it is combined with continuous clinical monitoring and, if necessary, an interventional radiology, its efficacy is more than $90 \%$.

This treatment fails in about $10-15 \%$ of cases, most often for extra-hepatic injury and less frequently for laparotomy

In the case of NOM hemostasis techniques are preferred over hepatic resection. If hemostasis is difficult to obtain, we should prefer periapical tamponation, rapid surgery to the concept of shorter laparotomy, multiclamping and venous venous shunt, and reintervention after 48-72 hours, management of secondary complications such as biliary fistulas or intrahepatic abscesses should be multidisciplinary, whether at the diagnostic or therapeutic level.

The overall mortality, with respect to the gravity of the anatomical hepatic injuries and associated injuries, is 10 to $20 \%$.

Keywords:liver, Trauma, biliary tract injuried, NOM, fistula Biliary, intrahepatic abscesses.

$O P-035$

\section{Blunt intestinal Trauma a retrospective assessment.}

\section{Agron DOGJANI ${ }^{1}$, Kastriot HAXHIREXHA ${ }^{2}$}

Author Information:

University Hospital of Trauma, General Surgery, Tirana, ALBANIA.

${ }^{2}$ University of Tetovo, General Surgery, Tetovo, RN of MACEDONIA

\begin{abstract}
Introduction:BAT is one of the most common causes of morbidity in that all age groups. Early identification of traumatic intestinal injuries (TInI) has been and remains a challenge to all trauma surgeons, as early finding and evaluation of their, it present difficulties of different natures, even with technological advances is a frightening for all surgeons because of morbidity and mortality that it carries. With all the different varieties of damage mechanisms InI of motor vehicle accidents remain the leading cause.

The first reports date for intestinal injuries we must go back to 1899 by Geille, who reported that intestinal injuries had $11 \%$ incidence in a group of patients who had undergone BAT. There are studies that said that intestinal injuries recently ranked third overall in the standings intra-abdominal injuries in BAT.

Identification of the mechanism of injury, the incoming data, the anatomical distribution of lesions, diagnostic methods, management and gastrointestinal damage results from BAT.

Material and Methods:The study includes patients with BAT in a 2-years period, that were introduced in the UHT, who underwent evaluation, treatment and management of gastrointestinal tract injury.

It is a retrospective study, patients were analysed in relation to the mechanism, time of presentation, in HdSt, anatomic localization of lesions, diagnostic methods, the tactics of treatment, complications, morbidity and mortality.
\end{abstract}

Results:Are taken into consideration about 21 (8\%) patients out of 257 which is the total number of patients with BAT in the study which had damage to the InI, they occupy $33 \%$ of cases treated with BAT street operators.

Age distribution is such 70 years old range 5years average 32.8, while such is distribution by age group; is 14 to 60 years old (6\%) followed by more than 60 years old (14.2\%) and finally by $0-14$ years old (23.8\%).

Conclusion:Early recognition of TInI from BAT is difficult, it's very important is their dangerousness be recognized because of its great potential complications affecting infection that gives directly to their morbidity and mortality.

Keywords:Blunt abdominal trauma, intestinal injury, colonic injury 
$O P-036$

\section{Do Indicators help in Manage Emergency Departments?}

Elizana PETRELA ${ }^{1,2}$, Edmond ZAIMI ${ }^{1,3}$, Albana KOÇIAJ ${ }^{4}$

Author Information:

${ }^{1}$ Faculty of Medicine, University of Medicine Tirana, ALBANIA

${ }^{2}$ Statistics Department, University Hospital Center "Mother Theresa" Tirana, ALBANIA

${ }^{3}$ Emergency Department, University Hospital Center "Mother Theresa" Tirana, ALBANIA Emergency Department, Hospital of Kavaja, ALBANIA

\section{Abstract}

Indicators are usually used to evaluate the service and to prepare for possible interventions. They serve as evidence of change and form an important part of most systems and approaches in the monitoring and evaluation process. Indicators can be calculated at different levels and defined in many different ways by different organizations. Indicators are defined as a quantitative or qualitative factor or a variable that provides a simple and reliable tool of measuring achievement, reflecting changes associated with an intervention, or helping to evaluate performance ${ }^{1}$.

An indicator is considered "as part of the information that provides evidence of a change" ${ }^{2}$. Evidence and change are two different things. An indicator is something that helps evaluate change, but is not change itself (even when the result is desired).

A good manager should know how to read the indicators correctly and then take other steps that lead to change (planning-designimplementation-re-evaluation).

$O P-037$

\section{Emergency Access and Management in the first 15 Minutes of Life Threats}

Basri LENJANI ${ }^{1 *}$, Nuhi ARSLLANI ${ }^{2}$, Esen UYSAL $^{2}$, Premtim RASHITI ${ }^{2}$, Nehat BAFTIU ${ }^{2}$, I BUNJAKU $^{2}$, GaniSHABANI ${ }^{3}$, M TAHIRI $^{3}$, Dardan LENJANI ${ }^{3}$, Erza VOCA ${ }^{3}$

Authors Information:
${ }^{I}$ Emergency Clinic, Hospital and University Clinical Service of KOSOVO

${ }^{2}$ Servise of Anestesia Clinical Service of KOSOVO

${ }^{3}$ Radiolgy Clinic University Clinical eof KOSOVO

${ }^{2}$ University Medical Centre Maribor, SLOVENIA

${ }^{2}$ International project and training cordinator of UrlaIzmir Provincial, TURKEY

\section{Abstract}

Trauma is a global, multidisciplinary problem caused by mechanical, chemical, physical and thermal factors and radiation. But in most cases, they are car accidents, various home accidents (minor, major) which can potentially be life threatening. Trauma is the sixth leading cause of death worldwide, resulting in five million or $10 \%$ of all deaths a year. Tracing injured traumas is a priority for treatment or transportation, according to their severity of injury. BLS in pre-hospital settings (primary, secondary, tertiary) and hospital ATLS requires a very multisystematic approach to be managed by US personnel. In hospital settings, trauma treatment is traditionally divided into two secondary, tertiary levels. Implementing a unified trauma algorithm facilitates access to diagnostics and treatment by carefully avoiding potential complications in order to reduce morbidity, disability and mortality. A proper medical trauma management team in pre-hospital, hospital settings educated and trained with basic and advanced trauma management courses and adhering to standard procedures and algorithms, protocols are key to the effective management of minor trauma injuries. and malaria which will affect the increase of quality of life as well as the reduction of morbidity, disability and mortality.

Keywords: Trauma, Injury, BLS, PhTLS, ATLS, Emergency department.

$O P-038$

Management of Acute Cardiac Failure in University Hospital Emergency Settings

Edmond ZAIMI $^{1}$, Albana KOCIAJ ${ }^{2}$.

Author Information:

${ }^{1}$ Emergency Department. University Hospital Center "Mother Theresa", Tirana, ALBANIA

${ }^{2}$ Emergency Department, Kavaja Hospital, ALBANIA 


\section{Abstract}

Acute cardiac insufficiency (ACI) is the rapid onset or worsening of CI signs and / or symptoms. It is a life-threatening clinical situation that requires immediate evaluation and treatment, which is generally followed by urgent hospitalization of patients.

The objective of this presentation is to describe the novelties in the diagnosis and treatment of ACI, including the use of cardiac markers to describe the advantages of Cardiac Markers-based therapy for the management of decompensated ACI; the role of different groups of preparations and non-invasive ventilation in the rapid treatment and stabilization of the situation, always in the light of the latest ESC and AHA clinical guidelines.

Emergency medical staff attitude from the first moment of contact with these patients, the order of actions and treatment of these patients until their placement, in the current conditions, based on the recommendations of clinical guidelines and our experience and experience, will be clarified in the presentation, with the aim of defining the same ACI management protocol.

OP - 039

\section{One Working Day in Emergency Center in Regional Prizren Hospital, Republic of Kosovo}

\author{
Sadri HULAJ ${ }^{1}$, Afrim JAHAJ ${ }^{1}$
}

Authors Information:

${ }^{1}$ Emergency Center, Regional Hospital in Prizren, KOSOVO
Abstract
Introduction: An Emergency Working Day presents more closely the medical services of care teams provided to all patients coming in or brought by emergency teams, accident rescuers, such as the Kosovo Police, other rescue teams or from Citizens themselves, in the most frequented hospital facility and of strategic importance. The care teams are in constant readiness but as needed increase the readiness and expand it with additional staff from other units of the Prizren Regional Hospital. In such cases the chain of survival comes to an end when early calls, immediate basic and advanced treatment provide

adequate medical treatment for each patient's need.

Material and Methods:As a source for completing this scientific work, are used documentation from Emergency Department books, medical reports, didactic photographs were used to make prospective uninterrupted work clearer and all medical cases (approximately 130 of them) were analyzed that have been seeking medical help for 24 hours. The time of arrival, the time of staying respectively in emergency services, examinations, necessary consultations as well as hospitalized and transferred cases at the Kosovo Main Emergency Center in Pristina were, they have been examined.

Results: One patient was resuscitated, 13 people treated cause thy had injuries on road traffic accidents, 5 cases of physical violence one of which was injured by a sharp tool, some patients injured by a height fail of approximately 3 meters, by motorcycle, bicycle, three poisoned patients, one of them with medicaments who attempted to commit suicide and 2 persons with alcohol overdose, 21 patients were treated with acute injuries, 26 were treated, one was transferred at the Kosovo Main Emergency Center for more advanced treatment... By age patients were of all ages but dominated by younger age groups (10-39 years old among the injured), by sex, most frequent patients were Male (ratio 3: 1). Time spent in observation (1- $2 \mathrm{~h}$ ). A number of patients were brought by ambulance, part of them by Kosovo Police (injured in physical conflicts) and some of them brought by family members as well as patients with mild disorders...

Conclusion: Arrival time in Emergency uninterrupted and no obvious night / day differences and sometimes several patients at the same time challenging teams to provide adequate treatment after proper professional treatment with teamwork and adequate consultation.

KeyWords:Patient, injury, doctor, treatment, observation, hospitalization

$O P-040$

Management of Syncope in University Hospital Emergency Settings

Edmond ZAIMI ${ }^{1}$, Enea SERANI ${ }^{2}$, Adelina XHEMALI $^{3}$ 
Author Information:

${ }^{1}$ Emergency Department. University Hospital Center "Mother Theresa", Tirana, ALBANIA

${ }^{2}$ Emergency Department, Regional Hospital of Gjirokastra, ALBANIA

${ }^{3}$ Family Physician Tirana, ALBANIA

\section{Abstract}

Syncope is a clinical syndrome, in which temporary loss of consciousness is caused by insufficient blood supply to the brain, often as a result of an immediate decrease in systemic arterial pressure (TA). Typical for the syncope is that this insufficient supply of blood to the brain occurs for a short time (810 seconds) and recovers spontaneously.

Syncope is a common symptom with which patients present with DU. Syncope is one of the many possible causes of temporary loss of consciousness (HPK). Most of these causes are benign and transient, but some are associated with increased morbidity and mortality, especially in old age.

What is the approach to syncopal patients, what are the opportunities to distinguish the causes of syncope episode, how to distinguish the true syncope from other causes of HPK, what are the main tests that help assess them, what are the initial concerns in treatmentrelated, how risk is determined and the attitude held in cases of indeterminate diagnosis, are the problems that will be elucidated in this presentation in light of recent ESC guidelines.

$O P-041$

\section{Pain Management for Pediatric Patients Contemporary Considerations}

Edmond PISTULLI ${ }^{1}$, Admir NAKE ${ }^{1}$, Faton KRASNIQI $^{2}$

\author{
Authors Information: \\ ${ }^{1}$ Faculty of Technical Medical Sciences, \\ University of Medicine in Tirana, Albania. \\ ${ }^{2}$ Neonatology department,University \\ Hospital Center, Prishtina-Kosovo
}

Relieving Pain in America. The International Association of the Study of Pain (IASP) defines pain as an unpleasant sensory and emotional experience associated with actual or potential tissue damage or described in terms of such damage Acute pain is the expected physiological response to a noxious chemical, thermal, or mechanical stimulus, and usually accompanies surgery, traumatic injury, tissue damage, or inflammatory processes. It is self-limiting and typically resolves over days to weeks, but it can persist longer as healing occurs. Chronic pain is defined as intractable pain that exists for three or more months despite adequate treatment.

For patients 18 years and younger, there do not yet exist specific consensus guidelines on prescribing opioids for chronic pain Pediatric practitioners are advised to use their best judgment when using opioids after appropriate use of nonopioid alternatives. Opioids are not usually indicated as a first line therapy for primary pain disorders [16]. However, current evidence suggests that opioid prescriptions should not be curtailed for moderately to severely painful conditions. Synchronal with this reconception, there are other new notions like nervous system hypersensitivity caused by the acute pain and the rehabilitation, known for their important role due to major developments in the multimodal analgesia. Last years the researches are mostly focused on pain assessments as the main element that precedes the treatment.

Conclusions: Multidisciplinary pain management, including pharmacological and integrative non-pharmacological therapies, has been demonstrated to be efficacious in the treatment of both acute and chronic pain.

Pharmacological interventions includeopioids and opioid-sparing agents that target specific aspects of the nociceptive and neuropathic pain physiology.

Keywords: Opioid reduction therapy, nonpharmacological therapy, cognitive behavioral therapy, hypnosis, mindfulnessbased stress reduction, acupuncture, pain rehabilitation.

$O P-042$

Temporary and Permanent Pacemaker in Acute Coronary Syndrome 
Ormir SHURDHA

Author Information:

${ }^{2}$ Cardiology Department, University Hospital

Center "Mother Theresa" Tirana, ALBANIA

\section{Abstract}

Background:Cardiac arrhythmias and conduction disorders are relatively common after Acute Coronary Syndrome (ACS). They are the result of autonomic stimulation, ischemia or necrosis of the conduction system. Two major factors determine the need for a cardiac output: symptoms associated with brady-arrhythmia or the site where conduction disorder has occurred.

During the ACS, different types of heart transplant surgeries may become apparent; sinus node impulse formation or conduction abnormalities, atrioventricular conduction (AV) disorders, and intraventricular conduction disorders.

Sinus rhythm disorders (sinus bradycardia, sinus arrest, exist sininoatrial) are more common, mainly during inferio-posterior stroke. Treatment is not necessary unless symptoms present; Atropine can be helpful. If bradycardia is prolonged and severe, or does not respond to atropine, a temporary cardiac arrest should be established. AV block occurs in $12-25 \%$ of patients with acute ACS.

The risk of progression from the first-degree block to the high degree AV block differs from the types of site of myocardial infarction. High-grade AV block, and especially after anterior wall infarction, indicates that damage is within the HisPurkinje system. Temporary emergency relief is required in these cases.

Modes and temporary cardiac arrest are transcutaneous and transvenous. Transcutaneous ligation is done by placing plates on defibrillation apparatus when the "Pacer Mode ON" option is selected. The transvenous suction is made through a suction probe placed in the right ventricle through a large vein, which is usually the femoral vein. The suction probe is connected to a transient pacemaker.

Keywords:Cardiac arrhythmias, Acute Coronary Syndrome, Pacemaker...
$O P-043$

\section{Syncope and Cardiac Arrhythmia in Emergency Department}

Indrit TEMALI $^{1^{*}}$

Author Information:

${ }^{1}$ Cardiology Department, American Hospital, Tirana, ALBANIA.

\section{Abstract}

Introduction; Syncope is a transient loss of consciousness due to cerebral globular hypoperfusion characterized by the first route, duration of Recently Spontaneous Recovery.Situations of patients presenting with syncope and cardiac arrhythmias in the emergency department pose a challenge for the emergency physician in deciding whether this patient should be hospitalized and if the situation presents a high risk, or can be managed alone to the emergency department and the patient then sent home.

Material and Methods; World Literature and professional experience

Results;Arrhythmias are prevalent in people of all ages and can occur in the context of a cardiac disease but also in a heart with normal structure. Every day we have a significant number of patients coming to the Emergency Department.

Conclusion; The diagnosis can be achieved by the correct implementation of the European Society of Cardiology (ESC) guidelines on syncope management. Regarding the prognosis it is necessary to stratify patients regarding the risk of short or long term performance. Furthermore, in the case of a syncope of undetermined nature it is important to determine the probability that this syncope has cardiac origin.

Keywords; syncop , diagnosis, cardia origin, Emergency department

$O P-044$

Treatment of Hydatid Cyst and BilioBronchial and Bilio-Pleuro-Bronchial Fistulas via Thoracotomy and Transdiaphragmic Approach from January 2005 to March 2019 in a Single Unit in Tirana Albania. 
Fadil GRADICA ${ }^{1}$, Lutfi LISHA ${ }^{1}$, Dhimiter

ARGJIRI $^{1}$, Alma CANI ${ }^{1}$, Fahri KOKICI ${ }^{1}$, Endri

SHEHU $^{2}$; Enton BOLLANO ${ }^{2}$, Dariel

THERESKA ${ }^{2}$, Ibrahim AVDI ${ }^{2}$, Helidon

NINO $^{2}$, Flora J. GRADICA ${ }^{3}$; V S. REXHA ${ }^{3}$

Authors Information:

${ }^{1}$ Thoracic Surgery Service, University Hospital "Shefqet Ndroqi" Tirana, ALBANIA

${ }^{2}$ Surgery Service University Hospital Centre"

Mother Theresa" Tirana, ALBANIA

${ }^{3}$ Public Pharmacy Service Tirana, ALBANIA

\begin{abstract}
Introduction: The aim of this study was to report the results of surgical treatment of hydatid bilio-bronchial et bilio-pleurobronchial fistulas via thoracotomy and trans diaphragmic approach.
\end{abstract}

Material and Methods:From January 2005 to March 2019, 32 cases were observed in the same center. Bilioptysis was the main symptom in $80 \%$ of cases. The diagnosis was based on chest radiography, thoracic and abdominal CT and abdominal ultrasonography, Fibro-bronchoscopy; all examinations visualized the cyst, intrathoracic collections, a diaphragmatic breach and biliary lesions. All patients were treated by one-stage thoracotomy one case by VATS left lower lobe. The procedures consisted of lung resection (lobectomy and/or segmentectomy) $(n=24)$ and decortication $(n=11)$ in the chest, cyst dome resection $(n=32)$ or part pericystectomy $(n=8)$ in the abdomen and suture and plastic of the diaphragmatic defect in all cases after hepatodiaphragmatic deconnection. An additional laparotomy was not necessary in all cases.

Results:There were 3 deaths (10\%):no one intraoperative death and three postoperative deaths, mostly related to pulmonary complications. Postoperative complications (14\%) were mainly respiratory. Clinical and radiological results were good with a oneyear follow-up.

Conclusion:Bilio-bronchial and bilio-pleurobronchial fistulas due to hydatid cyst are rare, but severe diseases. They are responsible for lesions at three levels: abdominal, diaphragmmatic and thoracic. A high perioperative mortality rate was observed. Thoracotomy with trans- diaphragmic approach is the best approach for surgical treatment at all three levels.

Keywords: Bilo pleural fistulae, biliobronchial fistulae, Thoracotomy.

$O P-045$

\section{Rib Fracture Fixation a New Era in Treatment of Chest Trauma in Albania}

\section{Fatmir ÇAUSHI}

Author Information:

Head of Department of Thoracic Surgery

University Hospital "Shefqet Ndroqi" Tirana, ALBANIA.
Abstract
Chest wall trauma now days are common, and contributes significantly to morbidity and mortality of trauma patients. Blunt trauma to the chest wall and rib fractures are remarkably
frequent and are the basis of considerable morbidity and possible mortality. Generalized management of multiple rib fractures and flail chest in last 50 years consists of adequate pain
control, management of pulmonary dysfunction by invasive and noninvasive means and in somecases, surgical fixation. Rib fractures can be interpreted as signs of significant trauma. Thegreater the number of fractured ribs, the higher the mortality and morbidity rates. Multiplestudies have shown that patients with flail chest have substantial benefit when they undergosurgery compared with nonoperative management.
Operative rib fixation has the potential to reduce ventilator days and ICU stay and subsequentlyhospital costs in selected patients with severe traumatic flail chest requiring mechanicalventilation. Rib-specific plating 
systems have started to be used in the last 10 years. These haveinaugurated in the modern era of rib repair with chest wall stabilization (CWS) techniques thatare safer, easier to perform, and more efficient. Recent consensus statements have sought todefine the indications and contraindications, as well as the when, the how, and the technicaldetails of CWS. Repair should be considered for patients who have three or more displaced ribfractures or a flail chest, whether or not mechanical ventilation is required.

Meanwhile in Albania, as in other fields, this protocol is entering so timidly facing prejudiced

thoughts and economic difficulties but with very convincing results. During the last 3 years, 10cases of chest stabilization have been treated in our clinic by positioning a specific plate systemfor the ribs. The result was excellent in 9 of them. These patients left the hospital after 7 days inexcellent condition. Meanwhile, in one case, three days after surgery an acute kidney failure wasinstalled which led to an unpleasant situation. However, even this separate case raises the issueof equipping our hospital facilities with the equipment needed to treat complex patients as a trauma patient.

Keywords:Ribs, fracture, fixation, surgery, technique.

$$
O P-046
$$

Post Traumatic Diaphragmatic Hernia. A Retrospective Study Based on Cases Between January 2012 - June 2019

\section{Ilir SKENDULI}

Authors Information:

Thoracic Surgeon, University Hospital "Shefqet Ndroqi" Tirana, ALBANIA

\footnotetext{
Abstract

Background: Diaphragmatic rupture is a lifethreatening condition, which may be due to severe penetrating or blunt trauma to the chest or abdomen. Considering that delayed diaphragmatic rupture has no clinical symptoms, results of diagnostic methods are restricted to the case report studies, also lack of early diagnosis of it in patients with multiple trauma and diversity of therapeutic methods, we decided to investigate the clinical
}

symptoms, diagnostic and therapeutic methods of patients with Delayed Diaphragmatic Rupture.

Material and Methods: In this retrospective study, we reviewed the data of all patients with diagnosis of delayed traumatic diaphragmatic rupture in surgical wards of "Shefqet Ndroqi" hospital in Tirana, Albania, between January 2012 and July 2019.

Results: Patients included in this study, 13 in total, where 10 (77\%) male and 3 (23\%) female. The age of the patients varied from 30 to76 years old. Traffic accidents were the most common cause of traumatic diaphragmatic rupture in our study (46\%). Dyspnea was the most common symptom in our subjects (68\%); Intestinal obstruction in 1 patient and Tension gastro thorax in 1 patient. $38.4 \%$ of our patients had right sided traumatic diaphragmatic rupture, and $61.6 \%$ had left sided traumatic diaphragmatic rupture. Colon was the most herniated organ. Chest $\mathrm{x}$-ray and computed tomography scan were used in all of our subjects for diagnosis of diaphragmatic rupture. The mean duration between trauma and surgery was $56.30 \pm 92.44$ months. The mean duration of hospitalization was $8.46 \pm 4.05$ days. The most common surgical method which was used for treatment of delayed diaphragmatic rupture in our patients was thoracotomy (92.3\%). In our recent study, primary treatment of diaphragm was performed in 12 patients, partial mesh in 1 patient. None of our subjects died.

Conclusion;Diagnosis of diaphragmatic rupture is difficult and has a clinical suspicion in high risk patients. So, using of radiological modalities for diagnosis and treatment of it is necessary. Moreover, delayed diaphragmatic rupture and diaphragmatic hernia should be considered in patients with recent blunt trauma and gastrointestinal or respiratory complaints.

Keywords: Diaphragmatic rupture; Mesh; Hernia;Traumatic.

$$
O P-047
$$

Traumatic Pneumothorax, Challenges of The Treatment (10-Year Study in Vlora Regional Hospital)

\section{Petraq MUSTAQE ${ }^{1}$, Lorenc GJOKA ${ }^{2}$}

Authors Information: 
${ }^{1}$ General Surgeon at Regional Hospital of Vlora, ALBANIA

${ }^{2}$ Anesthesiologist at Regional Hospital of Vlora, ALBANIA

\begin{abstract}
A common surgical disease is Pneumothorax which involves the Introduction of a quantity of air into the pleural cavity.

This pathology is, in most cases, traumatic and only in a small number comes from diseases of the pulmonary or mediastinal structures or organs.

Thoracic traumas are coming to light in our hospitals, this I owe more to motor vehicles than accidents that are not uncommon on our roads.

On the other hand, pneumothorax like the complication or the most common consequence of thoracic trauma is a common nosology that is often traumatic.

Our hospital Hospitalizes about 80 traumas involving thorax in one year, with a good proportion of tires after tension pneumothorax, hemothorax, rib fractures, wounds in this region, hematomas....

At 10 Years 2007-2017 Diagnosis 204 with Pneumothorax, to the scanning examinations that has improved to a good diagnostic extent and accurate.

The treatment of pneumothorax has been combined with hemothorax and / or open or blunt damages, whereas in two cases we have also been associated with chylothorax.
\end{abstract}

KEYWORDS: Pneumothorax traumatic, tension Pnx, hemothorax, chylothorax.

$O P-048$

\section{Treatment by Thoracotomy and VATS of Giant Bullous Emphysema}

Fadil GRADICA ${ }^{1}$, Lutfi LISHA ${ }^{1}$; Daniela XHEMALAJ ${ }^{1}$; Dhimitraq ARGJIRI ${ }^{1}$; Alma CANI $^{1}$; Fahri KOKICI ${ }^{1}$; Sali GRADICA ${ }^{1}$, Valbona S.Rexha ${ }^{1}$; Aishe R LALA ${ }^{1}$; Zef PERDUKA²,

Authors Information:

${ }^{1}$ Department of Surgery, Service of Thoracic Surgery, University hospital of Lung Disease Tirana, ALBANIA.

2Pneumology Service Rreshen Hospital, ALBANIA

\begin{abstract}
Introduction:There is general agreement that, with proper selection of patients with giant bullous emphysema (GEB) it is possible, using surgical methods, to improve lung function and consequently the quality of life.

Other patients may, sometimes, benefit from surgery in the management of specific complications such as pneumothorax or infection. This study was undertaken to assess the safety and efficacy of a VATS approach for GBE.
\end{abstract}

Material and Methods: From January 2006 to July 2019 we treatedthrough thoracotomy, 44 patients with giant bullous emphysema (GEB), and 12 patients through VATS. 17 female patients and 39 male patients,were with mean age 50 years old, (range 14- 72 years).

Results:We experienced 41 patients were presented with PNX and treated with pleurodesis. Two patients underwent a second operation by open thoracotomy because of a prolonged air leak.We have no operative death. Follow-up was 16 months range (136). In one patient we had prolonged aerorrhae and bilateral PNX.

One case with pleural empyema, a thoracic window was performed, from failure of lung.In three patients was done reoperation because of the receive.

Conclusions: Thoracotomy may be considered a suitable surgical technique and treatment of choice in GBE.VATS may be considered in nowadays as a suitable surgical technique as a mini-invasive surgery and in most of the cases it is more effective.

Keywords:Giant Bullae,Thoracotomy, VATS.

$O P-049$

Bilateral Chylothorax, after Blunt Chest Trauma. A case Report

Dorina SHTJEFNI ${ }^{{ }^{*}}$, Skender BUCI ${ }^{1}$, Sami $\mathrm{KOCEKU}^{1}$, Gjovalin BUSHI ${ }^{1}$, Sokol RUCI ${ }^{1}$, Kastriot SUBASHI ${ }^{1}$

Authors Information:

${ }^{1}$ Surgery Service, University Hospital of Trauma, Tirana, ALBANIA 


\begin{abstract}
Introduction:Traumatic bilateral chylothorax is a very rare occurrence. About $80 \%$ of chylothorax es are unilateral, in two-thirds of cases in right hemithorax. Appears with an inexhaustible pleural effusion in milk color, in abundance.

We will report the management of bilateral traumatic chylothorax as a very rare occurrence.
\end{abstract}

Material and Method: We present a patient with thoracic trauma. Fracture of thoracic vertebrae $8^{\text {-th }}$ to $10^{\text {-th }}$, with bilateral chylothorax. An Overview of diagnostic basis, treatment and evolution

Résults:A 36-year-old man has been the victim of a car accident, hospitalized in surgery with diag. Compressive fractures $\mathrm{Th}_{8}$ to $\mathrm{Th}_{10}$ andminimal pleural versament in the right side. Two days after the trauma on the scanning examination results, large amount of liquid in the right hemithorax and minimal liquid in the left hemithorax. From the drainage tube into the right pleural cavity flow about $1000 \mathrm{ml}$ of liquid with a milky appearance. Biochemical analysis of pleural fluid confirmed chylothorax.

We have performed Non- Operative Management, the first 10 days with a zerooral feeding, only parenteral nutrition, electrolyte, vitamins, plasma and albumin, followed by a fat-free diet. In CT reexamination 6 days after trauma resulted in an increased in the amount of fluid in the left pleural cavity, a drainage tube is inserted into the cystic pleural cavity from which approximately $700 \mathrm{ml}$ of milkycolored fluid is drained.

The drainage tube in the cystic pleural cavity is removed after 5 days. The fluid flow into the right pleural cavity persisted in an amount ranging from $150-300 \mathrm{ml}$ at $24 \mathrm{~h}$ for about 3 weeks. After this period for about 1 week the pleural effusion was in an amount of less than $100 \mathrm{ml}$ in 24 hours. In the fifth week, the drainage tube is removed after the leaks have been reduced to about $20-30 \mathrm{ml}$. The patient leaves the stabilized hospital.

CONCLUSIONS: The treatment is based on a fat-free diet. Chiral ligation of the thoracic ductus is indicated in a debit over $500 \mathrm{ml}$ at 24 hours, after 2 to 3 weeks
KEYWORD; Traumatic, chylothorax, milk color versament, nutrition parenteral.

$O P-050$

\section{Metamorphosis of a multicameral cyst of the humerus}

\section{Gjergj ÇAUSHI}

Authors Information:

University Medical of Tirana, Faculty of

Medicine, Tirana, ALBANIA

Head of Service orthopedic Surgery, University

Hospital of Trauma, Tirana, ALBANIA.

\begin{abstract}
Introduction:Multicameral bone cysts are fluid-filled benign cavities that expand over time, resulting in bone thinning. Usually, these cysts are reported in metaphysical areas of long bones with open physics. 85\% of them occur almost exclusively in children and adolescents, and are more aggressive in the first decade of life, and respectively, the recurrence rate for these patients is four times higher than for adolescents. Humerus and proximal femur make up almost $90 \%$ of these cases, they are classified as active when they are within $1 \mathrm{~cm}$ of the physique and latency as they progress to a diaphysis site. Differential diagnoses for them include aneurysmal bone cyst, fibrous dysplasia, enchondroma and intraosseous ganglia.

By the time skeletal maturity, most of them tend to resolve themselves. Non-operative treatment can be a valuable option for many patients with minor or symptomatic lesions. Interventions include steroid injection, open twisting and bone grafting, decompression and percutaneous injection of marrow or graft substitutes.
\end{abstract}

KEYWORDS: Unicameral bone cyst, Current concepts

$O P-051$

Indication, Technique, Available

Implants as Well as Alternative

Therapies for Periprosthetic Bone

Fracture Reconstruction.

Arjan MULLAHI

Author Information: 
Chef of Surgery, Ortopedy and Trauma Department St. Josef- Hospital Linnich, Rurdorfer Straße 49,52441 Linnich, GERMANY

\begin{abstract}
Introduction: Reconstruction of fractures and bone defects with traumatic and nontraumatic origin, with femuro-diapherese Strut Grafts represent a special And Great Treatment.
\end{abstract}

Materials and Methods: We will represent our revizion and personal results and a literature review.

Results: In literature is prescribed survey after Reconstruction with femuro diaphysere Strat Grafts, in a pursue within ten years, from the beginning of this technique at the 1991. A direct comparison of publication results it's difficult due to small number of cases in every publication, different types of implants that are used and heterogen patients. Another alternative treatment, different from Reconstruction with femuro diaphysere Strat Grafts is Tumorose Prosthesis, Gidlestone Situacion, ostheosynthesis with Locking Platte, alloplastic reconstruction and individual prosthesis $=3 \mathrm{D}$ printers.

Conclusion: Results of fractures and bone defects reconstruction with Femuro diaphysere Strut Grafts in short and long periods, are very satisfying and its advantage is for reconstruction stability, immediately immobilization of patients. Due to high requests in operating room about organization, long experience as a orthopedic surgeon this technique, unfortunately isn't as usual as we want to be.

Keywords: Femuro diaphysere strut Graft, periprosthesic fracture, allogene transplantate, bone banks, bone defects.

$O P-0052$

Physeal Bridge/Bar. A Serious Complication of the Distal Femoral Physeal Injury

Onder Murat DELIALIOGLU ${ }^{1}$, Ledian FEZOLLARI MD ${ }^{2}$, Kenan BAYRAKCI ${ }^{1}$

Authors Information:

${ }^{1}$ Orthopedic Services, American Hospital, Tirana, ALBANIA.

${ }^{2}$ Department of Orthopedic, University Hospital of Trauma Tirana, ALBANIA.
Abstract

Introduction:Long-bone fractures are common in children related with sportive activities or vehicular accidents and nearly one third of them involve the growth plate or physis. Isolated distal femoral physeal fractures are rare injuries accounting about $5 \%$ of all physeal injuries, and $50 \%$ of them result in some form of growth disturbance, and that increase with severity.

Fractures involving the physis fall into three main categories: fractures causing separation of the epiphysis, crossing the epiphyseal plate and injuries that crush the plate. The zone of provisional calcification and recently formed bone are the weak areas and are frequently the sites of injury.

Stable, truly nondisplaced fractures can be treated nonoperatively with long leg casting. There is a general consensus that displaced fractures should be operatively stabilized with internal fixation.

Late complications are more common than acute complications, and growth arrest is the most commonly observed complication.

A physeal bridge may develop after either operative or nonoperative treatment.

Physeal bar excision is recommended when less than $50 \%$ of the physis is involved, and there is adequate growth remaining (at least 2 $\mathrm{cm}$ or at least 2 years). Traditionally, fat has been used for interposition, but polymethylmethacrylate may be utilized as an alternative.

Physeal bar resection in isolation can lead to incomplete correction as the injured physis may still cease growing earlier than a healthy physis. A contralateral hemi epiphysiodesis or ipsilateral osteotomy may be needed later on. Therefore, patients must be followed closely in the years leading up to skeletal maturity.

Keywords: physeal fractures, physeal bridge, polymethylmethacrylate

$O P-053$

Surgical Management of Metastatic Lesions of the Proximal Femur with Pathological Fractures Using Intramedullary Nailing or Endoprosthetic Replacement.

Rezeart Dalipi ${ }^{1}$, Milan Samardziski ${ }^{1}$, Ilir Hasani $^{1}$, Antonio Gavrilovski ${ }^{1}$, Dalip Jahja ${ }^{1}$, Aleksandar Saveski ${ }^{1}$, Aleksandar Trajanovski ${ }^{1}$, Teodora Todorova ${ }^{1}$. 
Authors Information:

${ }^{1}$ University Clinic for TOARILUC, Skopje, Republic of North Macedonia

\begin{abstract}
Introduction:Metastatic neoplasms of bone are the most frequently observed malignant destructive bone lesions in adults. The proximal femur is the most commonly affected bone with metastatic disease in the appendicular skeleton. The orthopedic surgeon is often the first physician to see the patient with a pathologic fracture. The surgeon must be aware of the indications for intramedullary nailing (IMN), endoprosthetic replacement (EPR), as well as options for reconstruction, which are the two most commonly applied surgical methods used to treat the proximal femoral metastatic pathological fractures.

Purpose: The aim of the present reaview was to evaluate the clinical, functional and oncological outcomes for the better quality of life at the patients after surgically treatment of
\end{abstract}

proximal femur metastases with pathological fractures.

Material and Methods: Retrospectively were evaluated data of 28 patients with pathological fractures secondary to metastatic tumors of the proximal femur, treated at the Clinic of Orthopedic surgery in Skopje, in a period between April 2015 and June 2019.

Results:The mean age of the patients at the time of operation was 59.3 years (range: 4376-years). A total of 16 patients were treated with EPR and 12 patients were stabilized with IMN. The overall survival of patients with metastatic lesion and pathological fracture of proximal femur was dependent of the general condition and the amount of the metastases.

EPR is recommended for patients with relativelygood general condition and prognosis. IMNis best indicated when the patient's life expectancy is extremely limited.

Conclusion:Postoperative external beam irradiation can significantly reduce disease progression and subsequent loss of fixation. A multidisciplinary approach to this patient group will help optimize prognosis as well as function. The treatments are palliative but goals

include stabilize fractures, improve function and pain relief in an effort to achieve a better quality of life. Advances in surgical techniques and instrumentation have allowed more

effective life for the patients.

Keywords:proximal femur, pathological fractures, metastases.

$O P-054$

The “day - after" surgery: physiopathology after total hip replacement

Flamur VELLKU

Author Information:

Orthopedic Surgeon, Orthopedic Services, University Hospital of Trauma, Tirana, Albania.

\begin{abstract}
Introduction:In a total hip replacement (also called total hip arthroplasty), the damaged bone and cartilage is removed and replaced with prosthetic components. The damaged femoral head is removed and replaced with a metal stem that is placed into the hollow center of the femur.

Areas of concern in prevention and treatment of hip arthroplasty infection are presented, focusing on the pathophysiologic process involved. A review of the patient risk factors and the pathophysiologic action potentiating risk for infection include host immunity, nutritional status, diabetes, age, use of steroids or immunosuppressive drugs, rheumatoid arthritis, and urinary tract or other infections.

Recovery after surgery of total cochlear femoral prostheses improve outcomes and reduce hospital length of stay. This pre- and post-quality improvement study was designed to determine whether adding "non-surgical" components, to "existing" surgical components, in a healthcare setting, would improve patient recovery after total hip replacement.

In our study we have recorded the Total hip prothesis on the first 400 patients during period from October 2010-June2018in Orthopedic Services, University Hospital of Trauma.
\end{abstract}

Keywords:Femoral, prostheses, surgery. 
$O P-055$

\author{
Rehabilitation of Patients with Instability \\ Glenohumeral post Traumatic(non- \\ operative)
}

Manushaqe SARAÇI $^{1}$, Luljeta STANAJ ${ }^{1}$

Authors Information:

${ }^{1}$ Rehabilitation and Physiotherapy Service, University Hospital of Trauma, Tirana, ALBANIA.

\begin{abstract}
Introduction: The shoulder is composed of 3 bones and 4 articulatory surfaces, Glenohumeral Joint is the main one. Only 25\% of the head surface of the humeral makecontact with the glenoid. Glenohumeral joint instability is a common pathology often in the orthopedic. There are several factors to consider during the rehabilitation :1-Degree of instability, 2-Direction of instability (anterior, posterior, multidirectional), 3Concomitant pathologies, 4-Age and activity level of patient, 5-Arm dominance, 6-Rang of neuromuscular control.
\end{abstract}

Material and Methods: This is a prospective studyinvolving16 patients(13M,3F), that have been rehabilitated in our service during January 2018-June 2019. 12(75\%) patients had damaged right arm and 4(25\%) patients left arm. Average age $35^{+}$.- 6 years old. The rehabilitation protocol was applied in 4 phases: I - Acute phase (0-3 weeks), IIIntermediate phase (4-6 weeks), III Advanced strengthening (8-12 weeks), IVReturn to daily activities $12^{+}$. For measurements we used VAS scale, goniometer, MMT at the end phase of rehabilitation( $\left(1^{\text {st }}\right.$ day, weeks $3,6,12$,months 6,12).

RESULTS: 16 patients were taken into study. 6 patients were fully rehabilitated for 6 weeks. 8 patients were fully rehabilitated for 12 weeks.2 patients were rehabilitated for more than 12 weeks: 1 patient was with injured Rotator cuff and the other patient was neuro-muscular contracture.

Conclusions: It is very important to design a comprehensive program to establish full range of motion, balance capsular mobility along with maximizing muscular strength, endurance, proprioception, dynamic stability and neuromuscular control.
KEYWORDS: Glenohumeral joint, dynamic stabilization, neuromuscular control.

$O P-056$

\section{Nasal Fractures and Rhino-septoplasty.}

\section{Besim BOCI}

Authors Information:

Department of ENT Surgery, University Hospital Centre "Mother Teresa" Tirana, ALBANIA

\begin{abstract}
Introduction:The nose is the most prominent part of the face, and thus, most affected by facial trauma. Nasal bone fractures are reported to be the most common facial fractures and the third most common fractures in the human body.

Techniques for treating a nasal bone fracture are closed or open repositioning;

Closed repair is usually recommended within 3 weeks of injury is usually recommended. This is also because closed repository is a relatively simple procedure that can be performed in outpatient, under local anesthesia and is thus cost effective

However, results after closed repositioning are difficult to predict, and the ratios of external nasal deformity from post reposition vary from $9 \%$ to $50 \%$. Furthermore, some cases require open-label rhinoplasty at early stage.

Even a simple fracture can lead to a nasal deformity, and inadequate treatment can cause aesthetic and functional issues. External nose deformity prior to nasal trauma can lead to unexpected results. Therefore, the initial treatment should be carefully planned. In the present study, we aimed to analyse clinical features in cases where concurrent rhinoplasty and open landing were performed as primary treatment, after nasal bone fracture. Furthermore, we focused on patients who had external nasal deformity prior to nasal bone fracture.
\end{abstract}

Keywords:Nasal Fractures, Rhinoseptoplasty, outcomes, retrospective study.

$O P-057$

Ocular Globe Injuries in Children, a Retrospective Study in Albania. 
Marsida KRASNIQI ${ }^{1,2}$, Katerina KASA $^{3}$

Authors Information:

${ }^{1}$ University "Aleksander Moisiu” Durres;

ALBANIA

${ }^{2}$ International Eye Clinic, Tirana. ALBANIA

${ }^{3}$ Universitary Trauma Hospital Tirana. ALBANIA

\begin{abstract}
Introduction:Eye injuries are the leading cause of monocular blindness in children. At present, however, only limited follow-up studies exist. The aim of this study is to evaluate the clinical characteristics, visual outcome and prognostic factors of open globe
\end{abstract} injuries in children.

Material and Methods:The files of 38 patients aged 16 years or younger who had been treated for open globe injuries were examined. The type of injury was classified and the severity of injury was categorized in four grades.

Results:Our series included 5 eyes (13\%) with a rupture, 30 (79\%) with a penetrating and $3(8 \%)$ with a perforating injury. Follow - up varied from 3 days to 7 years (mean 15.5 months). The macula was attached at the last follow-up in all eyes. The final visual acuity ranged between 1.0 and light projection. In $47 \%$ of cases (18 eyes), the visual acuity was 0.5 ; $74 \%$ (28 eyes) achieved at least 0.1 . Eighteen of 38 eyes (47\%) had severe injury with posterior segment involvement. Visual acuity of at least 0.5 was achieved in $44 \%$ $(8 / 18)$ of these. Eyes with grade 2-4 injury treated with early vitrectomy had a final visual acuity of 0.5 in $58 \%$ of cases (7/13). Twenty -one (55\%) of 38 children were 8 years of age and eight of them (38\%) had a final visual acuity of 0.5 .

Conclusion:Saving the eye with an attached macula was possible in all eyes. Even in severely injured eyes good visual acuity can be established in about $50 \%$ of cases.

Keywords:Children, open globe, Albania, retrospective.

$O P-058$

\section{Lower Canal Laceration Treatment}

Bledar KRUJA, Elvis VELIU, Leodita

GJANA, Johana VRUHO

Authors Information:
Ophthalmology Department, University

Hospital of Trauma, Tirana, ALBANIA

\begin{abstract}
Lower canal laceration is a pathology that is not rare in ocular trauma. Although periocular injuries may involve the canal, the localization and extent of the injury they are not always evidenced at the first emergency examination. Complete examination includes damage assessment and canalicular test. The case in question is laceration of the lower palpebral canaliculus, a consequence of a car accident. This article will discuss the technique used in the treatment of canalicular injury.
\end{abstract}

Keywords: laceration, canal, trauma, injury.

$O P-059$

Reconstructive Surgery with free and Pedunculated Flap for Oral Cancer Defects: Our Personal Experience

Alfred $\mathrm{AGA}^{1 *}$, Emirjona VAJUSHI $\mathrm{MD}^{1}$, Armida KABA MD ${ }^{1}$, Rezarta KAPAJ ${ }^{2}$

Authors Information:

${ }^{I}$ ENT Department, American Hospital Tirana, ALBANIA

${ }^{2}$ Plastic Surgery Department, American Hospital Tirana, ALBANIA

Abstract

Introduction:The aim is to report our experience of using free and pedunculated flaps to repair intraoral defects, their reliability, reconstructive outcome and to assess the associated donor-site morbidity.

Material and Methods: The medical records of patients who had undergone a free flap reconstruction after radical resection of oral cancer between January 2014 and August 2019 at the American Hospital in Tirana were evaluated, to analyze morbidity of the surgery, hospitalization stay and the success rate of the flap vitality.

Results: In this period, 13 patients with oral cavity defects resulting from tumor resection underwent reconstruction with free flap. In four cases a radial forearm free flap was used 
for reconstruction of the lateral wall of the pharynx and base of the tongue after squamous cell carcinoma (SCC) resection. In another case, after resection of big tongue tumor that involves the floor of the mouth, we use anterolateral thigh flap. One scapular flap was used for reconstruction of hard palatal defect after total right maxillectomy for adenoid cystic carcinoma (ACC).

Conclusions: A free flap, in our experience, is ideal to repair intraoral defects. It is straightforward and reliable and has an acceptable reconstructive outcome. It might be a suitable procedure for patients that are inoperable before applying this technique in our country. The pedunculated flaps are useful and easy to harvest but we noticed that the reliability and the complication rate was higher. Proper intraoperative and postoperative care of the donor site can result in minimal morbidity.

Keywords:Free flap, reconstruction, radical resection, pharynx.

$O P-060$

\section{Trauma Management of the Oro Maxillofacial Region in Children.}

\section{Esat BARDHOSHI}

Author Information:

Department of Oro-Maxillofacial Surgery, Faculty of Dental Medicine

University of Medicine, Tirana, ALBANIA

\begin{abstract}
Introduction:Pediatric Oro maxillofacial trauma involving the bones of the face is associated with severe injury and disability. Although much is known about the epidemiology of facial fractures in adults, little is known about injury patterns and outcomes in children. The most common facial fractures were mandible, nasal and maxillary/zygoma. The most common mechanisms of injury are motor vehicle collisions, violence and falls. These fracture patterns and mechanisms of injury varies with age. Cranial and central facial injuries are more common among toddlers and infants,
\end{abstract}

and mandible injuries are more common among adolescents. Although bony craniofacial trauma is relatively uncommon among the pediatric population, it remains a substantial source of mortality, morbidity and hospital admissions. Continued efforts toward injury prevention are warranted.

Trauma in the Oro maxillofacial region in children are a frequent clinical reality. Due to physiological, anatomical, psychological features, etc., their treatment is relatively different from that of adults. These changes consist in details related to the elements of fixing the way of its realization, modifications of standard methods and follow-up of cases. that in most cases closed non-surgical conservative treatment is effective. Tracking of cases in progress is important to ensure the best anatomical development in both the dimensional and functional aspects. Physiotherapy remains an essential component of the treatment of patients in this group.

Keywords: Oro maxillofacial, trauma, Physiotherapy, treatment.

$O P-061$

Management of Traumatic Dental Injuries
in Emergency Departments.

Orjada GASHI ${ }^{1}$, Lorena HAXHIHYSENI ${ }^{1}$

Authors Information:

Orofacial Surgery Service, University Hospital of Trauma, Tirana, ALBANIA
Abstract
Introduction:Hospital emergency departments are confronted with managing dental emergencies of both traumatic and non-traumatic origin. However, the literature suggests inadequate knowledge of the management of traumatic dental injuries among medical professionals. The aim of the study was to evaluate the knowledge of ER physicians with different specialties, experience and hospital sectors for the management of avulsed teeth in the emergency rooms of Trauma University Hospital of Tirana. It also covers their attitude towards receiving further education on tooth avulsion management. 
Materials and Methods: A cross-sectional survey was conducted at the emergency rooms of Trauma University Hospital of Tirana. A self-administered questionnaire consisting of 31 multiple choice questions assessing both knowledge and attitude was distributed to the physicians who were working in the ER departments. Results: Response rate was $81.33 \%$. Data revealed that $58.9 \%$ of the respondents did not have prior knowledge about avulsion. $70 \%$ of participants showed willingness to replant the tooth, however, $60 \%$ would not do it by themselves. $40 \%$ of the physicians did not know the importance of extra-oral time. Saliva was selected as the best transport media for avulsed tooth by $40.1 \%$ of the participants. Regarding physician's attitude, $20 \%$ showed interest in receiving information about the subject.

Conclusion:Dentoalveolar trauma does not pose a significant morbid risk for the trauma patient. However, failure to recognize or obtain appropriate consultation can result in premature tooth or alveolar bone loss, resulting in problematic prosthetic rehabilitation, along with immediate and long-term emotional and social impact, especially among young adults. This study revealed that the majority of ER physicians lack the knowledge needed to manage avulsions cases. Hospital emergency departments are not confronted with managing dental emergencies of both traumatic and non-traumatic origin.

Keywords: Dental trauma, traumatic injuries, emergency department.

$O P-062$

\section{Chronic Rhinosinusitis}

Fatmir GUNI

Author Information:

ENT Service, University Hospital of Trauma, Tirana, ALBANIA.

\section{Abstract}

Chronic rhinosinusitis is persistent symptomatic inflammation of the nasal mucosa and paranasal sinuses. The clinic is dominated by 2 of 4 major signs: pain, facial pressure, hypo/anosmia, leakage and nasal congestion, which last more than 12 weeks and are evidenced by relevant examinations (anterior rhinoscopy, fibro-endoscopy and CT scanner)

With all developments that have been made in physiopathology over the last two decades, the exact etiology remains unclear because the causes are related to the subject and environmental factors.

Treatment consist in improving muco-ciliary clearance, treating infections and inflammation, improving sinus discharge and eliminating etiological factors. This treatment compromises a series of local, systemic and surgical therapies.

Keywords: rhinosinusitis, inflammation, treatment.

$O P-063$

Complex Bilateral Eye

TraumaManagement, a Case Report.

Katerina KASA ${ }^{1}$, Marsida KRASNIQI ${ }^{2}$, Etleva GJURASHAJ ${ }^{3}$

Authors Information:

1 University Hospital of Trauma, Tirana, ALBANIA

${ }^{2}$ University Aleksander Moisiu, Durres, ALBANIA

${ }^{3}$ Policlinici Nr. 9, Tirana, ALBANIA

\begin{abstract}
A 52 years old man was presented to the ophthalmic emergency room with a firework facial and eye trauma, on 31 December 2018. He presented multiple, small facial cutaneous abrasions and burn (Fig 1). His visus on the left eye was light perception and in the right eye was finger counting in $5 \mathrm{~cm}$. The clinical presentation of both eyes was lid and conjunctival burns, chemosis and conjunctival hemorrhage, corneal punctiform perforation, multiple corneal foreign bodies, hyphemia of 3mm, traumatic cataract (Fig 2). USG ( $\mathrm{A}+\mathrm{B}$ scan) revealed extended vitreous hemorrhage and a single foreign body. No irido dialysis was seen. The patient had no retinal detachment or optic nerve injury. After suturing and debriding the cornea, washing of the anterior chamber he underwent cataract surgery with scleral fixation intraocular lens, vitrectomy and silicone oil tamponade on both eyes (Fig 3). After 3 months' follow-up, his visual acuity on his right eye was 20/200 and his left eye was 20/400. The causes of
\end{abstract}


poor vision were corneal scar, astigmatism after corneal repair, vitreous hemorrhage, and macular edema. Fireworks-related eye injuries constitute an important cause of preventable blindness worldwide. As ocular firecracker injuries result in significant morbidity, public education regarding proper use of fireworks may help in reducing the incidence of ocular injuries.

Keywords:Eye trauma, fireworks, vitreous hemorrhage, traumatic cataract.

$O P-064$

\section{Analyzing Complications of Posterior Segment of the Eye after Contusion Injuries of the Eye}

Kenan LJUHAR, Aida GAVRANOVIĆ, Adem ZALIHIĆ, Mujo SUBAŠIĆ, Aner DURAKOVIĆ, Emir SMAJIĆ

Author Information:

Emergency Medical Service Center Sarajevo, BOSNIE HERZEGOVINA

\begin{abstract}
Introduction: One third of all eye injuries are contusion injuries. The most common causes of contusion eye injuries are objectives, a traffic accident. Complications of the eye injuries on posterior part are: haemophthalmus, ablatio corporis vitrei, optical nerve injuries, injuries of retina. Contusion injuries of the eye often causes a visual loss despite advance in diagnostic and surgical treatment. Treatment are proper examination and observation, curing with medicines, and VPP.

The Aims of this work are directed to identifying the most frequent cause of eye injuries; establish if there is a correlation between age, gender, occupation and complications of contusion injuries of the eye; establish if there is a correlation between type injury and complications of contusion injuries of the eye, finally, estabilish if there is correlation between visual acuity status and complication of contusion injuries of the eye.
\end{abstract}

Material and Methods: The study is retrospective, epidemiological, descriptive and analytical. The study included 84 examinees of both genders, hospitalized at the Department of Ophthalmology Clinical Center of the University of Sarajevo during the half year period in 2018., and whose diagnosis was contusion injuries of the eye. The material used for this study included patient records, disease histories and hospital release forms.

Results:The study results proved that there were more men 68 (82,26\%), than women 16 $(17,74 \%)$, with the most frequent age $40-59$ regardless the gender. Most frequent injuries occured in workers 22 (26,61\%). Wood was the mean of injury in $39(30,95 \%)$. Almost equally, both the right $(53,57 \%)$ and left eye $(44,04 \%)$ were injured, while in 2 patients $(2,38 \%)$ both eyes were injured at the same time. Of complication we found: haemophthalmus (21,43\%), ablatio retinae (13,09\%), commotio retinae (20,24\%), Purtscher retinopathy (3,57\%), blow-out fracture (3,57\%). Astigmatisms were found in 3 patients, as strabismus. Our research shown that $15(17,85 \%)$ patients needed surgical intervention. Percent of $66,67 \%$ patients were cured.

Conclusions: Eye traumatism is still one of the leading causes of vision loss. Contusion injury of the eye can result in serious morphological and functional impact in eye tissue structures and often causes a visual loss. Proper examination and appropriate approach are essential in successfull treatment and saving the visual functions.

Keywords: contusion injuries of the eye, eye traumatism, haemophthalmus, ablatio, vitrectomy.

$O P-065$

\section{Role of Ultrasound in Scrotal Emergencies}

Adham DARWEESH ${ }^{1}$; Maysa MOHAMED

Authors Information:

${ }^{1}$ Senior Consultant RadiologistHead of Body Imaging, HGH,Associate Program Director, A/Professor of Clinical Radiology, Weill Cornell Medical College, Hamad Medical Corporation, Doha, QATAR.

\begin{abstract}
Introduction: The purpose of the presentation is to demonstrate the role of Ultrasound (US) in the evaluation of patients with acute scrotum, especially in cases where immediate surgical intervention is crucial as
\end{abstract}


in testicular torsion and scrotal trauma. In such clinical situations, US can provide accurate diagnosis and testis can be saved. Scrotal US is regarded as the imaging modality of choice for evaluation of acute scrotum in both pediatric and adult population. It plays important role in triage of patients presenting to the Emergency Department with scrotal pain and offers timely and accurate diagnosis of these cases.

Materials and Methods: Review of scrotal ultrasound in Emergency Department setting based on our own experience and the available literature.

Results: We discuss proper techniques of scrotal ultrasound (gray scale \& color Doppler), anatomy, normal and abnormal findings relating to different scrotal pathologies, with special emphasis on trauma, torsion, orchitis and tumors.

Conclusion: Clinical history along with color Doppler ultrasound help to achieve accurate and timely diagnosis of scrotal pathologies allowing for rapid intervention to salvage the testis, particularly in cases of torsion and trauma.

Keywords: ultrasound, scrotum, emergency, diagnosis.

$O P-066$

Family Physicians and Ultrasound Trends in Everyday Practice

Erinda KOSTURI ${ }^{1}$, Aurora KRASNIQI.

Authors Information:

${ }^{1}$ Family Physician Polyclinic No 3, Tirana, ALBANIA.

\begin{abstract}
Introduction:Ultrasound examination is one of the most common adjuvant examinations often required in primary care practice by family physicians. But is it always appropriately ordered? Should it be performed in their offices rather than in other facilities? Would it help if primary care physicians were educated on imaging modalities?
\end{abstract}

Materials and Methods:In collaboration with family physicans we reviewed clinical charts and studied different cases, by anamnesis, medical reports and evaluated positive and negative findings in the radiological report.

Results: Files of 163 patients were gathered during a 5year period. Of these, $58.9 \%$ were females and $41.1 \%$ were males.

Of the requested ultrasound examinations, less than $45 \%$, contained any conclusive information. Abdominal ultrasound was the most likely to be ordered by physicians and also to be reported as normal after the scan.

Conclusions: These findings suggest that it is of great importance to educate primary care physicians about imaging referral. The main problem faced during everyday practice, when it came to ultrasound referral remained the need to satisfy patients request to undergo this imaging modality, because it was perceived as a safe, radiation free, easy and fast modality.

KEYWORDS: Ultrasound, family physician, radiological report.

\section{$O P-067$}

\section{Errors in Imaging of Patients with Traumatic Injuries}

Admir MUSTAFA $^{1 *}$, Vidi DEMKO ${ }^{1}$, Kujtim ALIMERI $^{1}$, Sonja BUTORAC (SARACI) ${ }^{1}$, Gëzim GURI ${ }^{1}$, Artan GJIKA ${ }^{1}$, Najada KALLASHI $^{1}$, Bardhyl VEIZI ${ }^{1}$

Authors Information: ${ }^{1}$ Department of Radiology, University Trauma Hospital, Tirana, ALBANIA.
Abstract
Introduction: The advent of multi-detector computed tomography hasdrastically improved the outcomes of patients with multiple traumatic injuries. Emergency and trauma care produce a "perfect storm" for radiological errors: uncooperative patients, inadequate histories, time-critical decisions, concurrent tasks and often junior personnel working after hours in busy emergency departments.
However, there are still diagnostic challenges to be considered. A missed or the delay of a 
diagnosis in trauma patients can sometimes be related to perception or other non-visual cues, while other errors are due to poor technique or poor image quality. The main cause of diagnostic errors in the emergency department is the failure to correctly interpret radiographs, and the majority of diagnoses missed on radiographs are fractures.

Missed diagnoses potentially have important consequences for patients, clinicians and radiologists. Radiologists play a pivotal role in the diagnostic assessment of polytrauma patients and of patients with non-traumatic cranium - thoracic - abdominal emergencies, and key elements to reduce errors in the emergency setting are knowledge, experience and the correct application of imaging protocols.

In order to avoid any serious complications, it is important for the practicing radiologist to be cognizant of some of the most common types of errors.

The objective of this presentation is to review the various types of errors in the evaluation of patients with multiple trauma injuries or polytrauma and the spectrum of diagnostic errors in radiography, ultrasonography and CT in the emergency setting.

Keywords: Imaging, errors, trauma,diagnostic.

$$
O P-068
$$

\section{Imaging of Blunt Pancreatic Trauma: The Value of Initial and Sequential CT Examinations}

Adham DARWEESH ${ }^{1}$, Maysa MOHAMED, Wojciech SZMIGIELSKI

\section{Authors Information:}

${ }^{1}$ Senior Consultant RadiologistHead of Body Imaging, HGH,Associate Program Director, A/Professor of Clinical Radiology, Weill Cornell Medical College in Qatar Hamad Medical Corporation, Doha, QATAR
Abstract
Introduction: Blunt traumatic pancreatic injury is uncommon, frequently associated with other injuries and can pose diagnostic challenge particularly in early pancreatic

injury where CT findings can be subtle. Computed Tomography (CT) is generally used as first line imaging modality for detection of visceral injuries in polytrauma patients. Several direct and indirect CT signs can be seen in patients with blunt pancreatic injury. The purpose of the presentation is to review CT findings in blunt pancreatic trauma, highlight the value of repeated CT scan in these cases, and correlate CT findings with endoscopic retrogradecholangiopancreatography magnetic resonance cholangiopancreatography ultrasound (US), laboratory and surgical findings.

Materials and Methods: Data was retrospectively collected covering a period of eight years and analyzed. A total of 21 patients (17 males and 4 females) with blunt pancreatic injury were identified. All cases had CT on admission, and 15 had follow up CT performed between 24 hours and 14 days later. 9 cases had US, 8 cases had ERCP and MRCP was performed for one case. Serum amylase level was obtained on admission in all cases.

Results: Initial CT performed on admission was positive in 17 patients (81.0\%); and diagnosis was missed in 4 patients (19.0\%). In these four cases, a repeated CT scan was found positive. ERCP demonstrated rupture of the main pancreatic duct in 7 cases. One MRCP was positive. Elevated serum amylase was found in 14 cases (66.7\%). Specific CT features depicted on the initial and repeated examinations were organ fracture 33.3\%, swelling - 38.1\%, haematoma/contusion $38.1 \%$, fluid between splenic vein and pancreas $-19.0 \%$.

Conclusion: Proper CT technique and accurate interpretation of CT findings are essential for diagnosis of pancreatic injury. $\mathrm{CT}$ also plays important role in following up and managing complications in these cases. If initial CT on admission was negative in a symptomatic patient with elevated serum amylase, follow up CT preferably within 2448 hours should be performed. 
Keywords:pancreatic, blunt, trauma, CT scanner.

$O P-069$

Role of Multi-detector Computed

Tomography in Bowel and Mesenteric

Injury

Parambath Arif NELLIYULLA 1; Maysa MOHAMED, Adham DARWEESH ${ }^{2}$

Authors Information:

${ }^{2}$ Senior Consultant RadiologistHead of Body Imaging, HGH,Associate Program Director, A/Professor of Clinical Radiology, Weill Cornell Medical College in Qatar Hamad Medical Corporation, Doha, QATAR

\begin{abstract}
Introduction: Bowel and mesenteric injury (BMI) is the third most common type of injury resulting from blunt abdominal trauma (BAT), seen in approximately $5 \%$ of BAT patients undergoing laparotomy. Multidetector Computed Tomography(MDCT) is considered the diagnostic modality of choice for the evaluation of hemodynamically stable patients who sustained blunt abdominal trauma. These injuries are however frequently missed on CT and diagnosis remains challenging.

The aim of this presentation is to evaluate role of Multi-Detector Computed Tomography (MDCT) in bowel and mesenteric injury following blunt abdominal trauma and highlight CT findings and potential pitfalls.
\end{abstract}

Materials and Methods: Radiological reports of MDCT examinations of patients with bowel and mesenteric injury over a three-years period were retrospectively identified and relevant data were collected and analyzed.

Results: Different specific and less specific CT findings of bowel and mesenteric injury were illustrated, without the use of oral contrast.
Conclusion: Multi-detector CT is an excellent diagnostic modality for the evaluation of bowel and mesenteric injury. Knowledge of the CT findings of bowel and mesenteric injury would improve detection rate and allow for accurate diagnosis and management. Administration of oral contrast agent is not mandatory for initial evaluation of patients with such injuries.

Keywords:CT scanner, bowel, mesenteric, injury.

$O P-070$

The Accuracy of Imaging Techniques in Early Detection of Breast Disorders

\section{Albana SHAHINI}

Author Information:

Radiologist, Americal Hospital, Tirana, ALBANIA

\section{Abstract \\ INTRODUCTION:Screening is key to early detection and treatment of breast cancer in women.To assess the accuracy of imaging techniques in early detection of breast disorders (benign or malign) and the importance of follow-up protocols.}

MATERIALS AND METHODS: This is a cross-sectional study that brings evidence on the sensitivity (SN), specificity (SP) and accuracy (ACC) of mammography (MM) and ultrasound (US) examinations, as compared to the biopsy findings (gold standard). Some 254 women underwent MM and US examinations, and biopsies were taken for 39 women with BIRADS 4 and 5.

Results: We found a decrease (100\% to $90 \%)$ in the SN for US benign findings as the breast density increased and an uneven variability on US SN for malign findings. The SP for all US findings was $100 \%$. The US ACC decreased for benign findings and increased for malign findings.If a woman has malign findings in $\mathrm{MM}$, odds to have a positive malignant finding in biopsy increase 20 times $(p=0.008)$. This increase is 12 times for women with an $\underline{\mathbf{U S}}$ positive malignant finding $(\mathrm{p}=0.005)$. The odds for having a biopsy positive malignant finding increase 24 times for women that have at least one malignant 
finding in either MM or US. The odds for having a biopsy malignant finding increase 18 times if the lesion is $1.5 \mathrm{~cm}$ or larger $(p=0.018)$. If women have both a malign finding (in either MM or US) and the lesion is over $1.5 \mathrm{~cm}$ then odds to have a biopsy positive malignant finding increase to 21 times more for malign lesions over $1.5 \mathrm{~cm}$ $(p=0.027)$ and 8 times $(p=0.027)$ for $M M$ or US findings.

Conclusion: Out study confirms the crucial importance of using MM and US to early detect breast cancer on women, enabling so the early treatment and saving lives. Benefits are higher if these examinations are used in combination annually for women aged 40-50 years old and biannually for those over 50 . Confirmations using biopsy should come for all those women BIRADS 4 and 5 as well as for BIRADS 3 with lesion that increased since last check-up. Especially for women with denser breasts (ACR 3 and 4), it is vital to have both examinations (MM and US) used in combination as US has a higher ACC in this group and is irreplaceable for ages 5155 years old.

Keywords:Breast, cancer, imaging.

$O P-071$

\section{Role of CT in Acute Abdomen Especially in Appendicitis Acuta}

Behar TOCILLA, Bledi CEKREZI, Arben DHIMA, Albana SHAHINI, Adriatik DAKU, Fjorda TUKA.

\section{Authors Information:}

Radiology Service, American Hospital, Tirana, ALBANIA

\footnotetext{
Abstract

This segment of the presentation will go over the optimal imaging approach for patients presenting with acute abdominal pain. CT findings will be emphasized. Key imaging findings of non-traumatic causesof acute abdominal pain including gastrointestinal like appendicitis will be explained. A systematic approach for the imaging evaluation of patients with abdominal emergencies will be illustrated and explained including proper scan protocols and analysis of imaging findings.
}

This presentation will be an approach to imaging diagnoses of patients with acute abdominal pain, where computed tomography will be emphasized.

Key imaging data of cases of acute appendicitis will be mentioned.

A systematic evaluation of the imaging data and explanation of the examination protocols. KEYWORDS: Imaging, abdominal pain, examination, protocol.

$O P-072$

\section{Acute Epiploic Appendagitis an unusual cause of Abdominal Pain}

\section{Dorina SHQALSHI}

Author Information:

Radiologi service, Salus Hospital, Tirana, ALBANIA

Abstract
Inttroduction:Primary epiploic appendagitis is a rare condition that occurs after either torsion or spontaneous venous thrombosis with subsequent development of infarct and inflammation.

It is determined by a benign, self-limiting inflammatory or ischemic damage of the epiploic appendages, or it may be secondary to other inflammatory conditions affecting adjacent abdominal organs. The condition most commonly manifests in the $4^{\text {th }}$ to $5^{\text {th }}$ decades of life, predominantly in men.

The diagnosis of epiploic appendagitis has a clear clinical relevance and significant implications in patients' management. In case of acute abdominal pain, this entity is considered a clinical mimicker of other conditions, such as acute appendicitis and diverticulitis, that usually require a surgical evaluation. Imaging plays an essential role in the diagnosis of epiploic appendagitis, allowing the appropriate detection and differential diagnosis, in order to avoid relevant misdiagnosis.

Before the use of the CT scan, most of the cases were diagnosed during surgery.

Once the diagnosis of epiploic appendagitis is made, conservative treatment must be initiated to avoid unnecessary surgery.

Surgical treatment is reserved for patients in whom conservative treatment fails or for patients whose signs and symptoms worsen. 
Keywords:appendagitis, torsion, venous thrombosis, Abdominal Pain

$O P-073$

\section{Acute Airway-Threatening Presentations of Thyroid Diseases in very old Patients}

Carlos MESQUITA

Author Information:

General and Emergency Surgery
Departments - Trauma Center
Coimbra Central and University Hospital,
PORTUGAL

Abstract

Acute airway-threatening from thyroid diseases is not common but may occur due to giantgoiters, hematomas and thyroid malignancies, among others. The author underlines the need for the surgeon working in acute care setting to be familiar with thyroid surgery.

Three recent cases, in very old patients, of acute airway-threatening from thyroid diseases are described:A 91-year-old male with known giant right-sided thyroid mass, with a cytologic study suggestive of follicular tumor, who had refused thyroid surgery two months earlier, was hospitalized for acute exacerbation of dyspnea and hoarseness. Cervical mass extended from the hyoid bone to the upper mediastinum, with left shift of cervical structures. Right thyroidectomy and istmectomy were urgently performed.

A 88-yeard-old female with a known bilateral large cervical mass was admitted due to stridor. Computed tomography revealed a large retrosternal compressive goiter with a remaining tracheal lumen of $2 \mathrm{~mm}$. Surgical risk was significantly aggravated by a severe aortic stenosis. Since there was no place for a classic tracheostomy and endotracheal intubation could be difficult or impossible, everything was prepared in order to anesthetize the patient under ECMO, which, however, did not become necessary.
Intubated with the aid of a fiberscope, a total thyroidectomy was possible, after partial sternotomy.

A 78-year-old female complaining of stridor and dysphagia, from a large right cervical mass, with a $4 \mathrm{~mm}$ tracheal lumen was admitted. A tracheostomy was performed but, due to airway obstruction not completely resolved by this procedure, a right lobectomy with isthmectomy was performed urgently. Although cytologic study was suggestive of papillary carcinoma, the degree of local infiltration, evident at the time of surgery, first drew attention to the possibility of an anaplastic thyroid cancer.

Acute airway-threatening from thyroid diseases is not common. Early recognition of theseconditions and urgent, partial or total thyroidectomy may be necessary to alleviate symptoms, when tracheostomy is not an effective option. The surgeon working in an acute care environment should be aware of these possible scenarios and be prepared to solve them.

$O P-074$

Cold nodules of Thyroid Glands.

Myzafer KAÇI, Arvit LLAZANI, Arvin DIBRA, A. HOXHA, Astrit XHEMALI, Artan BODEC, Ilir SKENDULI, Armir DAMZI.

Authors Information:

1 General Surgeon, University Hospital Center "Mother Theresa" Tirana, ALBANIA
Abstract
Introduction: A cold nodule is a thyroid nodule that does notproduce thyroid hormone. This type of nodule may be malignant or benign. In older women the cold nodules have a chance of $15 \%$ to be malignant but this percentage increases in children and men especially if they have been in contact with ionizing radiation. On scintigraphy cold nodules do not show but are easily shown on ultrasound. The levels of calcium and the thyroid hormone in blood are used to diagnose the nodules. To verify the nature 
and the probability of malignance the best preoperative testing method is cytopuncture of the nodule. The final result is given by the postoperative biopsy.

Materials and Methods:A prospective study is conducted in 80 selected patients who do not have any other pathology and have been under medical treatment for the thyroid nodules. The age of the patients varied from 25 to 65 years old. 48 patients (60\%) were females and the other 32 (40\%) were males. All the preoperative testing methods are used such as Thyroid Gland Ultrasound; Scintigraphy of the gland; Thyroid hormones and calcium levels in blood and also Cytopuncture of the nodules. The authors attempted to emphasize the comparison between the preoperative data and intraoperative findings and the biopsy results of the cold nodules of thyroid gland.

Results: The Calcium blood level had no changes in testing preoperative and postoperative. Preoperative testing diagnosed $100 \%$ of the patients under study with cold nodule of thyroid gland. The most affected age is 35-54 and females have a higher probability of disease. Postoperative testing resulted in $2.5 \%$ of the patients with papillary thyroid carcinoma.

Conclusions: Interventions in early ages are necessary to prevent goiter and the use of iodine salts. It is necessary to know the prevalence of the disease so that proper health policies can be developed for the future. The best way to diagnose Thyroid Gland Carcinoma is the postoperative biopsy.

Keywords:cyto-puncture, Ultrasound; Scintigraphy, gland Thyroid

$$
O P-75
$$

\section{PneumomediastinumAfter Thyroidectomy A Case Report.}

Alma LLUKAÇAJ ${ }^{1}$, Etmont ÇELIKU ${ }^{1}$, Majlinda NACO ${ }^{2}$, Maksim BASHA ${ }^{3}$, Ilir SKENDULI $^{4}$, Fatmir CAUSHI ${ }^{4}$, Ilir $\mathrm{PEPOSHI}^{5}$

Authors Information:

${ }^{1}$ Sugery Department at University Hospital Center

"Mother Theresa", Tirana, ALBANIA

${ }^{2}$ Anestesiologist at University Hospital Center

“Mother Theresa”, Tirana, ALBANIA
${ }^{3}$ Radiologist at University Hospital Center

"Mother Theresa", Tirana, ALBANIA

${ }^{4}$ Torakal Service, at University Hospital "Shefqet

Ndroqi", Tirana, ALBANIA

${ }^{5}$ Pneumology Service, at University Hospital

"Shefqet Ndroqi", Tirana, ALBANIA

\begin{abstract}
Introduction: Pneumomediastinum is caused by an injury in lungs level, trachea or esophagus. If there isn't a clear cause then probably is a spontaneouspneumomediastinum. It's very rare ( 1 in 7.000 $35.000)$ and it is found more in males then females. Age 20-40 and patients with lungs diseases or asthma have higher risk.
\end{abstract}

Materials and Methods: We present a case report, treated in First Clinic at Surgery Service at University Hospital Center "Mother Theresa".

Results:It is a female patient, 5 years old, with diagnosis: Multinodular Struma. A total thyroidectomy was realized, with bilateral recurrent nerve conservation. 40 hours post operation, the patients represent with progressive edema of face, neck and upper part of chest. After CT scanner, we noticed a pneumomediastinum. The treatment was with drain chest tube, that was placed in operation wound, antibiotics and anticoagulants. Patient discharge hospital after 15days.

Conclusions:Pneumomediastinum is a rare condition and has serios consequences. Conservative treatment and observation are necessary to ensure patient improvement. Keywords:pneumo-mediastinum, thyroid, surgery, bronchoscopy, fibrogastroscopy.

$O P-076$

\section{Strangulated Giant Para-oesophageal Hernias}

\section{Carlos MESQUITA}

Author Information:

General and Emergency Surgery Departments, Trauma Center, Coimbra Central and University Hospital, PORTUGAL
Abstract
Paraoesophageal hernias (PEH) are mediastinal displacements of abdominal organs andoccur due to a lax diaphragmatic hiatus and phreno-esophageal 
membrane.PHEoccurrence and size increase with age. They account for approximately 5$10 \%$ of

diaphragmatic hernias. If symptomatic they can present with intermittent nonspecificsymptoms, including epigastric pain, postprandial fullness, chest pain, nausea, iron

deficiency anemia, gastro-esophageal reflux or dyspnea. In patients without prohibitive operative risk they should be surgically corrected, avoiding the risk of an acute and potentially life-threatening complication (incarceration, strangulation, volvulus, ischemia, perforation or gangrene), when emergent surgical repair is required. The risk of developing these complications is less than $2 \% /$ year and the mortality rate associated is approximately $5 \%$.

To highlight the need of a high suspicion index in uncommon clinical conditions and the value of an experienced team, a case of an acute complication in a misdiagnosed incarcerated giant $\mathrm{PEH}$ is presented.

An 86-year-old female with multiple medical conditions, including hypertension, chronic renal failure on hemodialysis, diabetes mellitus and a previously known large PEH, was admitted in our emergency department due to acute abdominal pain, vomiting and hypotension. Blood results revealed an isolated white cell count of 18.0x109/L. CTangio was performed and ruled out mesenteric ischemia.

$O P-077$

Acute Liquid Thorax as a Result of the Pancreato-pulmonary Fistula. A Case Report.

\section{Kenan KARAVDIĆ}

Author Information:

Clinic for Pediatric Surgery, Clinic Center of University Sarajevo, BOSNIAHERZEGOVINA

\footnotetext{
Abstract

Introduction:Pseudocyst formation and pancreato-pleural fistula are a rare complication of acute and chronic pancreatitis.

Case Presentation: A 10-year-old girl was admitted to our pediatric emergency department with an acute onset of dyspnea.
}

On physical examination, she was found to be dyspneic and tachypneic and having dullness to percussion with decreased breath sounds on the left hemithorax. Chest X-ray, computed tomography and magnetic resonance imaging demonstrate the existence of a multilocular lesion in the left hemithorax region that compresses the mediastinum and pushes it to the right position, pancreas with calcium zones and a liquid zone that can correspond to a pancreas pseudocyst. The patient was successfully treated with combined operative (thoracotomy), invasive (percutaneous puncture of pseudocysts of pancreas) and conservative therapy. Conclusion:Pancreato-pleural Fistula (PPF) is an uncommon complication of chronic pancreatitis leading to large and recurrent pleural effusion. The condition is diagnosed with very high pleural fluid amylase, Rtg and $\mathrm{CT}$. We recommend a conservative treatment of chronic pancreatitis in combination with percutaneous drainage of pseudocysts of the pancreas, thus reducing the accumulation of fluid in the thorax.

KEYWORDS: Pancreato-pleural Fistula; Pseudocyst; Pancreas

$O P-078$

Open Fractures of Cruris and Treatment with External Fixator.

Ilir HASMUCA*, Zamir DEMIRAJ

Author Information:

${ }^{1}$ Orthopedic Service, University Hospital of Trauma, Tirana, ALBANIA

\begin{abstract}
Inroduction: Open fractures of the ankle are fractures caused by high-energy forces and observed in the young population. They constitute the backbone of all open fractures. The incidence is 1 per 5000 fractures and 23 cases per 100,000 persons. Men are the most affected by open fracture of the ankle. These fractures are highly susceptible to infections and delays in bone healing and therefore require rigorous and very careful treatment.

Material and Methods: This is a casecontrol study on cases and controls for selection over a 3-year period 2016-2018. Only the cases treated by Orthopedic Clinic 1
\end{abstract}


at the University Trauma Hospital for the period January 2016-December 2018, with Diagnosis: Aperta Cruris Fracture, were studied. Data on both cases and controls were obtained from the University Trauma Hospital of Tirana. In this study we defined (operational definition) cases as "Open fractures treated with external fixator" and as controls "Open fractures treated with other or untreated techniques".

Results:Open fractures of the ankle are more common in males than in females where the average age is 41 years. $95.3 \%$ of invoices are type I, II Gustilo / Anderson and $4.7 \%$ are type III Gustilo / Anderson.

Hospital stay and post op a day higher in cases of external fixator use. Meanwhile, timing operation has no significant differences between operating techniques.

Conclusions: The use of an external fixator facilitates better management of your wounds and facilitates the design of the ultimate treatment arm, but this does not mean that other surgical techniques are ineffective. The use of other surgical techniques is effective in Gustilo / Anderson type I / II fractures, while the use of the fixator is very effective in type III Gustilo / Anderson fractures.

Keywords: Open fractures of the ankle, external fixator

$O P-079$

Anterior Cruciate Ligament Reconstruction our Thirty-Five Years' Experience.

Alan ANDONOVSKI $^{1 *}$, Ilir SHABANI ${ }^{1}$, BiljanaANDONOVSKA, Ilir HASANI ${ }^{1}$

Authors Information:

${ }^{1}$ University clinic for orthopedic surgery, traumatology, anesthesiology and intensive care, Skopje, Republic of North MACEDONIA

\footnotetext{
Abstract

Introduction: Anterior cruciate ligament (ACL) reconstruction is a common operative procedure which is not predictably successful in restoring the patients pre-injury state. Only $60-70 \%$ of patients resume their previous level of activity in $20.6 \%$ after 10 and $51.7 \%$ after 20 years of the patients with ACL reconstruction, knee osteoarthritis is developed. Reasons can be either the wrong
}

perception that ACL reconstruction is a routine surgical procedure that can be performed even by orthopedic surgeons performing less than 10 procedures in a year or poorly defined parameters of healing and rehabilitation needed to successfully return patients to pre-injury levels of function.

The solution can be in the individualization of the surgery and rehabilitation to meet each patient's needs. That means that no single surgical technique can address all needs or situations successfully and that different variables like: timing of surgery, the type of used graft material, the number of grafts, graft fixation and additional extra-articular surgery should be customized to address the needs of the patient. New methods able to enhance tissue healing, speed up recovery time, and decrease the risk of ACL graft failure are also investigated.

We want to present our thirty-five years' experience with ACL reconstruction, starting from year 1984 since today. We tried different ACL reconstruction surgical techniques, we kept what was good and changed what gave bad results. A La Carte surgery or individualize each surgery for each patients the main principle for us today.

Keywords:Anterior cruciate ligament reconstruction, Different ACL surgical techniques, Thirty- five years' experience.

$O P-080$

Dilemmas in the Decision-Making Process in the Treatment of Proximal Femur Fractures - Metanalysis of Papers Done at Several Different Centers

Antonio GAVRILOVSKI ${ }^{1}$, Rezeart DALIPI ${ }^{1 *}$, Aleksandar TRAJANOVSKI ${ }^{1}$. Aleksandar SAVESKI $I^{1}$

Authors Information:

${ }^{1}$ Orthopaedic Surgeon University Clinic for TOARILUC, - Skopje, RN of MACEDONIA

\footnotetext{
Abstract

Proximal Femur Fractures are frequent injuries in the patient population of every trauma centre and have a high incidence in the general population. Paralleling trends of demographic

forecasts, their incidence will continue to rise in the future.
} 
Due to the still undefined views and controversies regarding the treatment of proximal femur fractures, there is a need for meta-analysis of published papers.

The aim is to draw conclusions that will help form an appropriate decision about the type of treatment for these fractures and thereby provide the most appropriate treatment for each patient.

We list 7 metanalyses with a significant number of cases covered and an appropriate follow-up period, as well as our own experience in our center.

Keywords:Fracture, femur, type of treatment.

$O P-081$

Patellar Tendon Tear Treatment in Athletes.

Edvin SELMANI ${ }^{1,2}$, Ylber ZAMA ${ }^{2}$, Valbona SELMANI $^{2}$, Gjergji CAUSHI ${ }^{1,2}$

Authors Information:

${ }^{1}$ Faculty of Medicine, University of Medicine Tirana, ALBANIA.

${ }^{2}$ Department of Orthopedic, University Hospital of Trauma Tirana, ALBANIA.

\begin{abstract}
Introduction: Patellar tendon tear is easy to diagnose but is still often overlooked. The aim of this study was to assess early and late results of surgical treatment of acute patellar tendon rupture with Krakow technique.
\end{abstract}

Material and Methods: A retrospective study included 5 patients (5 males). Mean age was $29.6 \pm 9.9$ - years. Lesions comprised 4 tendon body ruptures, Tendon repair was achieved with Krakow technique. Results were evaluated in 2 steps: on patient files at a mean follow-up of 7.1 months (range, 3-24 months) to assess complications and early functional and radiological results; and by phone at a mean follow-up of 2.3 years in order to assess long-term functional outcome on Lysholm score and patient satisfaction.

Results: Four knees were assessed at a mean 7.1 months. Mean knee flexion was $128.5^{\circ} \pm$ $7.5^{\circ}$ (range, $85^{\circ}-150^{\circ}$ ), extension $-1^{\circ}$ (range, $-15^{\circ}$ to $0^{\circ}$ ) and Caton-Deschamps index 0.96 (range, 0.57-1.29). All knees were further assessed at a mean 2.3 years. Mean Lysholm score was 93.7 points (range, 61-100). All patients were satisfied or very satisfied with the result. All had returned to their previous job, and sports activities.

Conclusion: Patellar tendon rupture has good prognosis if diagnosis and surgical treatment is early.

Keywords: Acute rupture; Patellar tendon; Surgical repair.

$O P-082$

\section{Chronic Achilles Tendon Tear Treatment}

Edvin SELMANI $^{1,2}$, Ylber ZAMA ${ }^{2}$, Valbona SELMANI $^{2}$, Gjergji CAUSHI ${ }^{1,2}$

Author Information:

${ }^{1}$ Faculty of Medicine, University of Medicine

Tirana, ALBANIA.

${ }^{2}$ Department of Orthopedic, University

Hospital of Trauma Tirana, ALBANIA.

\begin{abstract}
Introduction: This study aimed to evaluate the long-term follow-up results of $\mathrm{V}$ Y tendon plasty with fascia turndown, for repairing chronic Achilles tendon tears.

Matherial and Methods: Seventeen patients (12 males, 5 females), who were diagnosed with chronic Achilles tendon rupture and met the inclusion criteria, were included in the study. These patients received treatment by means of $\mathrm{V}-\mathrm{Y}$ tendon plasty with fascia turndown from January 2005 to December 2011. Clinical outcomes of the patients were assessed by using isokinetic strength testing, questioning the patient regarding residual discomfort, pain, or swelling and having the ability to perform heel rises and using American Orthopaedic Foot \& Ankle Society's (AOFAS's) Ankle-Hind Foot Scale score. Mean follow-up duration was 10 years (7-13 years).
\end{abstract}

Results: Mean time from the injury to operative treatment was 6 months. Mean operative defect of Achilles tendon in neutral 
position after debridement was $5 \mathrm{~cm}$. During the follow-up, the mean calf atrophy was 3.4 $\mathrm{cm}$. The mean 30 degrees/s plantarflex and 120 degrees/s plantarflex peak torques were 89 and $45 \mathrm{Nm}$, respectively. The mean 30 degrees/s plantarflex peak torque deficiency was $16 \%$. The mean 120 degrees $/ \mathrm{s}$ plantarflex peak torque deficiency was $17 \%$. The average peak torque deficiency was $17 \%$. The pre- and postoperative mean AOFAS Ankle-Hindfoot Scale scores were 64 and 95, respectively. No patient had a rerupture. Superficial wound infection was treated with oral antibiotic therapy in 2 patients (11\%).

Conclusions: The V-Y tendon plasty with fascia turndown forrepair chronic Achilles tendon ruptures yielded results comparable with the literature regarding clinical outcomes. This method did not require synthetic materials for augmentation and was an economic alternative compared to other repair methods.

Keywords:Achilles Tendon,Tear,Treatment, tendon plasty.

$O P-083$

Minimally Invasive Treatment ESIN of Tibial Fractures During Sport Injuries

Lazar TODOROVIC ${ }^{1 *}$ Marjan KAMILOSKI $^{1}$, Anila RACAJ ${ }^{1}$, Slavica LITAJKOVSKA $^{1}$, Natalija COKLESKA ${ }^{1}$, Zllato ALEKSOVSKI ${ }^{1}$

\section{Authors Information:}

${ }^{1}$ University Clinic of Pediatric Surgery, Medical Faculty, University of St. Cyril and Methodius, Skopje, Republic of North MACEDONIA.

\footnotetext{
Abstract

Introduction:The fractures of tibial shaft are common during some sports. Their treatment is primarily conservative, but certain types of fractures require operative stabilization.

The aim of this study was to present our experience and results from the flexible intramedullary nailing of unstable tibial
}

fractures during sport injuries, treated at our Clinic in the period between 2005 and 2014.

Material and Methods:One hundred and eight patients, aged from 14 to 18 years were treated with primary intramedullary nailing for a tibial shaft fracture. The functional outcome was assessed by the KarlstromOlerud scoring system at 16 weeks after the surgical intervention.

Results:Seventy-two (78\%) patients had excellent result, eleven (16\%) good and six (6\%) patients had acceptable functional outcome.

Conclusion:Flexible intramedullary nailing ESIN for tibial fractures, enables early weight bearing, provides good functional results and is accompanied with a low rate of postoperative complications. It is a method of choice for irreducible and unstable tibial fractures during sports injuries, especially in teen athletes.

Keywords:Sport injuries, tibial shaft fractures, ESIN, functional outcome, teen athletes.

$O P-084$

Anterior Interosseous Nerve Syndrome

Following Paediatric Supracondylar Humeral Fracture: Case Report

Teodor PEVEC ${ }^{1}$, Simona KALŠEK ${ }^{1}$

Authors Information:

${ }^{1}$ General Hospital Ptuj, SLOVENIA

\begin{abstract}
INTRODUCTION: Anterior interosseous nerve (AIN) is a motor branch of the median nerve that runs deep into the forearm. It innervates three muscles of the forearm. Their isolated palsy is known as the AIN syndrome. Distal forearm fractures are some of the most common fractures in children, associated with frequent neurovascular complications. The paper describes a relatively rare complication in our everyday medical practice: an AIN syndrome following a supracondylar fracture.
\end{abstract} CASE PRESENTATION: A 5-year-old boy fell off a swing and broke his forearm. It was a supracondylar fracture. No neurological disorders were described. Manual reduction 
and K-wire fixation were performed. At a follow-up visit, the mother pointed out that the child was unable to flex the thumb of the treated arm. We recognized the AIN syndrome.

Discussion: The AIN syndrome was most likely a consequence of the injury to the elbow itself. Due to our focus on more frequent neurological disorders, especially those of the ulnar nerve, the AIN syndrome was detected relatively late.

Conclusion:In the preoperative and especially in the postoperative care of children with distal forearm fractures, precise assessment of the patient's neurological status is required for an early detection of associated injuries.

Keywords:Nerve injury, child, distal humerus fracture, complication, treatment.

$O P-085$

\section{The Human Entorhinal Cortex}

Sadi BEXHETI, Jeton SHATRI, Veton

ADEMI, Dijon MUSLIU

Authors Information:

Medical Faculty - University of Tetova, $R N$ of MACEDONIA

\section{Abstract}

Introduction: The aim of this work was the cytoarchitectonic analysis of the modular organization of the entorhinal area of the human cortex. Investigations were carried out out postmortem material, on both parahypocampal gyruses obtained from the brains of 22 adult people aged between 25 and 85 years. All the brains were physiologically normal, without any signs of neuropathologic diseases. Firstly, the brains were fixed in $10 \%$ neutral formalin, lasting from 30 to 45 days. Then the parahypocampal gyruses were embedded in celloidin, cut in series along the tangential plan and stained according to the Nissl method. Graphic reconstruction of cytoarchitectonic units of the II layer was carried out on 12 brains. A total of 520 sections was analyzed.

The results show that the existence of cytoarchitectonic units in the form of bands and columns of stellar neurons are basic morphological properties of the entorhinal area. It was established that regional differences exist in the distribution, shape and density of cytoarchitectonic units (columns and cell bands). Significant differences were found between individual bands and columns in width, length as well as in the numeric density of cells within individual bands. Such a complex picture of cytoarchitectonic modules may be called a modular "mosaic". One of the most interesting findings of this work was the existence of statistically significant differences in the numerical density of stellar neurons and the area of columns between the right and left hemispheres. All these findings indicate the existence of the three-dimensional mosaic of cytoarchitectonic modules in the human entorhinal area. The study and the change of the modular mosaic in various neurological diseases, particularly those accompanied by memory disorders, enable a new approach to the path neurobiology of the brain cortex.

Keywords:cytoarchitectonic, brain cortex, stellar neurons

$O P-086$

Anatomical Variations of Posterior Part of the Circle of Willis in Kosovo

Agon TURKAJ ${ }^{1}$, Antigona KABASHI ${ }^{2}$, Jeton SHATRI $^{3}$, Sadi BEXHETI ${ }^{l}$

Author Information:

${ }^{I}$ Faculty of Medicine, University of Pristina, Pristina - KOSOVO

${ }^{2}$ Clinic of Radiology, University Clinical Center of Kosovo, Pristina - KOSOVO

${ }^{3}$ Clinic of Radiology - University Clinical Center of Kosovo, Institute of Anatomy at the Faculty of Medicine, University of Pristina, Pristina KOSOVO
Abstract
Introduction: The posterior cerebral artery- P1 (PCA-P1) and posterior communicating cerebral artery (PCoA) has been noted in literature to have anatomical variations. Variations and anomalies of the PCA-P1 and PCoA have several clinical implications; including assisting in 
understanding the clinical signs of a stroke.

The aim of this study was to describe the anatomy and anomalies of the PCA-

P1 and PCoA.

Materials and Methods: This is

an observative descriptive study performed at the University Clinical Center, Clinic of Radiology in Kosovo. A randomized sample of 454 angiographic examinations in adult patients of both sexes without clinical manifestations for cerebrovascular disease who were instructed to exploration is included.

Results: In the present study posterior part had a complete structure in $26.6 \%$ of the cases. More completed is presented in female (30.7\%) than males $(22.55 \%)$ and variant $\mathrm{E}$ in $45.8 \%$. While absence of either the ACOP or the two sides (type D, E, G, H) is represented in $62 \%$ of the examined patients.

Conclusion: Anomalies of the PCAP1 and PCoA have a great significance since it forms a link between two major arterial systems the internal carotid and the vertebrobasilar system. The knowledge of this variation is very important during surgical and radiological interventions. Keywords: variations of posterior part, arteries, posterior cerebral artery-P1.

$O P-087$

\section{Pains Problems, Pain as a Notion.}

\section{Pirro PRIFTI}

Author Information:

Faculty of Technical Medical Sciences, European University of Tirana, ALBANIA

\section{Abstract}

Pain is a subjective symptom and evaluation is subjective. We also can evaluate pain in objective way, in acute pain where it can compromise vital signs, such as arterial pressure, pulse, oxygen saturation and respiratory frequency. Pain is associated with emotional and physiological response. Pain is a complex problem, involving both the mind and the body. For instance, backpainhas no known cause in most cases, and stressful life events can make it worse. But that doesn't mean it isn't real. Pain is an invisible problem that others can't see, but that doesn't mean it's all in your head. There are countless options for pain relief. They include relaxation techniques, exercise, physical therapy, overthe-counter and prescription medications, surgery, injections (into muscle, joints or your back), and complementary treatments such as acupuncture and massage. It may not always be possible to completely get rid of your pain, but you can use many techniques to help manage it much better. Health careproviders begin with a conservative approach to pain relief and prescribe non-narcotic pain-relief medications, which are not addictive. Doctors may prescribe narcotics, such as codeine and morphine, if pain becomes severe, such as when treating cancerpain. Many people fear that they will become addicted to narcotics. Physical dependence is not the same thing as addiction. And, physical dependence isn't a problem as long as you do not stop taking the narcotics suddenly. Addiction to narcotics is not usually a problem, unless you have a history of recreational drug or alcohol addiction.

Keywords:Pain, objective, subjective, treatment.

\section{$O P-088$}

\section{Principles in Treating Neonatal Meningitis}

Albi ARKAXHIU ${ }^{1 *}$, Desli SHAHINI ${ }^{2}$

Author Information:

${ }^{1}$ Neonatolog, Kavaja Municipal Hospital, ALBANIA

${ }^{2}$ General Physician, Medical Researcher, General Health Coordinator at WHO, ALBANIA

\begin{abstract}
INTRODUCTION: Newborn babies are at a much higher risk of developing meningitis than any other age group, as their immune system is weaker and more susceptible to viruses and illnesses. Since the bacteria that causes meningitis can be transferred from mother to baby, prenatal care and virus screening for the disease is extremely important. Meningitis happens when a viral
\end{abstract}


or bacterial infection spreads to the spinal fluid and the brain fluid. Whereas viral meningitis can typically resolve on its own without treatment, bacterial meningitis is a serious illness that can lead to brain damage, hearing loss, and even death.

Materials and Methods:During our study in the Maternity Hospital "Koco Gliozheni”, in the period 2017-2019 we have used three categories in classifying: Clinical symptom of newborn baby, gender, laboratory analyses. Difference between clinical symptom of newborn and mum is also important. In continue of those categories we have proposed treatment starting from the behavior and procedure of neonatology and all the specific fight against microorganism that had caused it. Effective interventions and documentation of responses to these interventions will receive increased scrutiny.

Conclusion: Most mothers do not know that they have Group B Strep. In fact, doctors say that $40 \%$ of women carry Group B Strep in the lower intestines, anus, and vagina without even knowing it -there are no symptoms that indicate that the mother has the bacteria in her system.

By testing anywhere between weeks 35 and 37 of pregnancy, the doctor should be able to identify whether the mother has Group B Strep and to be able to put her on safe and pregnancy-friendly antibiotics in enough time that the bacteria may be gone in time for delivery.

The combination of a good therapy before with an experienced neonatology that know better the way of treat a baby that comes from an infected mother may lead us to less cases of infected neonatal babies.

KEYWORDS: neonatal meningitis, symptoms and laboratory, treatment, emergency.

$$
\text { OP-089 }
$$

\section{Anaphylaxis Related Posttraumatic Stress Disorder}

Vrenos HODAJ $^{1}$, Desli SHAHINI ${ }^{2}$
Authors Information:

${ }^{1}$ Internist, Emergency Department Trauma University Hospital of Trauma, Tirana, ALBANIA

${ }^{2}$ General Physician, Medical Researcher, WHO health coordinator, Tirana, ALBANIA

\begin{abstract}
Introduction: Post-traumatic stress disorder (PTSD) is a chronic and disabling entity, which negatively impacts health and quality of life. It occurs after events that are perceived as life-threatening and has a lifetime rate of $7 \%$. Anaphylaxis is an acute systemic reaction with symptoms of an immediate-type allergic reaction which can involve the whole organism and is potentially life-threatening. The relation in between PTSD and anaphylaxis is one of the main challenges in emergency department.
\end{abstract}

Materials and Methods: Specific patient subgroups-including different ages and genders, trauma presentations with anaphylaxis correlated with. Also, the anaphylactic shock increases one's risk of developing PTSD, and for this we did the survey in causes of reactions. Also, we have surveyed the symptoms in both genders, ages and different trauma presentation. Also, the treatment is going to be evaluated for each group symptoms. This is a study made in the emergency department University Hospital of Trauma. There are 190 cases taken for the study during the 2017-2019. Effective treatment and documentation of responses to this process are recorded for our study. It is a great result to see positive results on patients that were suffering from both syndromes.

Results: A total of 190 subjects were enrolled with an average age of 48 yrs. The cases are all PTSD related with anaphylaxis. 52\% of them are verified in our study that PTSD has strong relation with anaphylaxis and is the main risk factor of its development. $98 \%$ of them had been well recovered after the treatment, but all of them had the same symptoms, that affect breathing system, blood circulation and heart. Males after 50 years old and women during menopause had the most aggressive symptoms. Anaphylaxis is well presented in mostly females, and it is life threating event that has been present even before in their life. It is our duty as doctors to be sure that such events are going to happen less, in future. 
Conclusions:Retrospective studies suggest that up to $1 \%$ of patients present to the emergency department of a maximum care hospital because of an anaphylactic reaction. It is specially represented in female, in comparison with males.

One to three anaphylaxis-induced fatalities per year per 1 million inhabitants are estimated. Our study suggests that PTSD rates are increased in individuals with a history of anaphylaxis. Therefore, routine follow-up of these individuals should include surveillance for this disorder.

Keywords: PTSD, anaphylaxis, treatment, survey, emergency.

$O P-090$

\section{Abdominal Trauma and Conservative Follow Up}

Akgün ÇELIK

Author Information:

General Surgeon, Istanbul, TURKEY

\section{Abstract.}

Introduction;Injuries within the abdominal cavity and abdominal organs continue to be a problem for general surgeons. Abdomen is the most common region to be injured following the head and extremities Injuries may be in the form of blunt abdominal trauma, stab wounds or gunshot wounds. The majority of blunt abdominal trauma is seen after motor vehicle accidents. There have been major changes in the approach to abdominal trauma in the last 20 years.

Non-operative treatment strategies are becoming more common. Conservative treatment experience is based more on experience in blunt abdominal trauma. However, recent publications suggest that nonoperative (conservative) treatment can be performed in gunshot and stab wounds in selected patients. Most of the abdominal trauma patients are younger than 40 years, and it remains to be an important cause of morbidity-mortality in this population

In our clinics, hemodynamically stable patients without any signs of peritonitis underwent conservative treatment for their abdominal injuries. These patients were followed up closely with physical examination by the same physician and by imaging methods. Informed consent was obtained from all patients. Blunt abdominal trauma patients who were hemodynamically stable and without any signs of peritonitis were followed nonoperatively. Patients were operated if deterioration of hemodynamic stability and/or lesion (hematoma, etc.) progression on imaging was detected. In addition to this, additional methods such as diagnostic laparotomy or diagnostic laparoscopy were used for diagnosis and monitoring, especially in noncooperative patients due to various reasons, including cranial trauma or alcohol. Patients with suspicion of hollow organ injuries were also operated.

Keywords: Abdominal injury, abdominal trauma, nonoperative treatment

OP-091

Management Penetrating Abdominal Stab Wounds

\section{Engin GÖÇMEN}

\section{Author Information:}

General Surgeon, Istanbul, TURKEY

\begin{abstract}
To review the results of our treatment approach to penetrating abdominal stab wounds and to reduce unnecessary hospital stay.
\end{abstract}

Material and Methods: Admission files and surgery notes of 87 patients with penetrating abdominal stab wounds, who had applied to Gureba Bezmialem Valide Sultan Hospital Emergency Clinic between 2008 and 2010, were reviewed retrospectively. The results physical examination of the abdomen, and laboratory findings, such as amylase level, leukocyte count and hematocrit value, were evaluated. Comparison of the data was made by Fisher's exact test for categorical data and t-test was used for continuous data. Results: A total of 87 patients were treated in our emergency clinic with the diagnosis of penetrating abdominal trauma and 48 of them were operated on. 19 of 36 patients with positive laparotomy findings had a high white blood cell count, hematocrit value decreased in 16 patients, and only 2 patients had high 
amylase values. Out of the surgically treated 48 patients, 9 patients had hypotension and tachycardia. Negative laparotomy findings were found in two of them.

Conclusion:Our negative laparotomy rate could have been $59 \%$ instead of $33 \%$ if the patients would have been operated on just because of penetrating abdominal wounds.

KeyWords:Stab wounds, conservative, laparotomy

$O P-092$

\section{Nonoperative Management in Pediatric Splenic Injury. A Case Report}

Elona MARKECI ${ }^{1}$, Admir MUSTAFA ${ }^{1}$, Afron $\mathrm{MICI}^{1}$, Skender BUCI ${ }^{1}$

Author Information:

${ }^{I}$ Department of Surgery University Hospital of Trauma - Tirana, ALBANIA

\section{Abstract}

The risk of overwhelming post splenectomy infection (OPSI) prompted the evolution toward preservation of the injured spleen. Nonoperative management (NOM) of blunt injury to the spleen in adults has become the standard of care in hemodynamically stable patients. This modality of treatment began in the 1970's in pediatric patients. It is highly successful with overall failures rates from $2 \%$ to $31 \%$ (average $10.8 \%$ ) - with the majority of failures occurring in the first 24 hours.

The spleen is one of the most commonly injured organs in the body after blunt abdominal trauma. Our objective is to present a case of pediatric splenic injury in hemodynamically stable patient was managed non-operatively. In the past splenectomy was the standard procedure for traumatic blunt splenic injury, when bleeding of the spleen occurred.

Since the spleen performs important immunological functions the advantage of a spleen-saving approach is preservation of immunological functions. Especially in the pediatric population splenic preservation is an important objective. The risk of overwhelming post splenectomy infection (OPSI) prompted the evolution toward preservation of the injured spleen. We present a case of a 12 -year-old male with a history of falling down on his left flank playing games with friends. He was evaluated and proved to have splenic rupture and hemoperitoneum. He was offered conservative treatment.

Keywords: Pediatric,spleen, injury, blunt trauma.

$O P-093$

Total Rupture of the Caput Pancreas after Blunt Abdominal Trauma. A Case Report and Review of the Literature.

Skender BUCI $^{1}$, Dorina SHTJEFNI ${ }^{1}$, Sami KOCEKU $^{1}$, Gjovalin BUSHI ${ }^{1}$, Sokol RUCI ${ }^{1}$

Authors Information:

${ }^{1}$ Surgery Service, University Hospital of

Trauma, Tirana, ALBANIA

\begin{abstract}
Introduction:Isolated pancreatic lesions are rare, often difficult to identify, they account for $0.2-6 \%$ of abdominal trauma. The treatment strategy is related to the integrity of the Wirsung Canal. The purpose of this paper is to report the management of a pancreatic trauma with rupture of the major pancreatic duct
\end{abstract}

Material and Methods:We present a patient with complete rupture of the pancreas without duodenal injury from a closed abdominal trauma. A 41-year-old man has been the victim of multiple aggressions with his fists and legs, in the abdomen, with echoes in the epigastric region. The patient is hospitalized for trauma surgery 4 hours after the aggression with abdominal pain. Examination found, hemodynamically stable, a sensible abdomen, lipazemia and amilazemia were normal. The scanner has shown a rupture in the pancreatic head region (Class III Lucas), liquid in the omental and periapical bursa. The patient was admitted to the operating block 6 hours after trauma. Surgical exploration has shown hemoperitoneum, complete rupture of the pancreatic head, rupture of the major pancreatic duct, duodenum and other abdominal organs had a preserved integrity of major pancreatic duct, hemostasis and suturing at the level of the injured area of the pancreas, a wash and abdominal drainage. Post-operative evolution was favorable and without complications. On 
the 10th day after the intervention the patient was removed from the healing hospital.

Conclusion:Imaging has an essential role in the evaluation of pancreatic damage before the onset of clinical and biological signs. The treatment of pancreatic trauma associated with major pancreatic duct injury is surgical. The prognosis depends on a rapid diagnosis and surgical intervention.

Keywords:pancreatic lesions, abdominal trauma, major pancreatic duct

OP -094

\section{Abdominal trauma and Liver some Management Considerations after a Retrospective Study.}

\section{Agron DOGJANI ${ }^{1}$, Kastriot HAXHIREXHA ${ }^{2}$}

Author Information:

IGeneral Surgery Service, University Hospital of Trauma, General Surgery, Tirana, ALBANIA.

${ }^{2}$ University of Tetovo, General Surgery Service, Tetovo, RN of MACEDONIA

\footnotetext{
Abstract

Introduction:The liver is the most frequently injured abdominal organ. Most of liver injuries are relatively minor and heal spontaneously with nonoperative management, which consists of observation and possibly arteriography and embolization. Purpose to describe the causes of trauma, the degree of injury, the chosen method of treatment, the success rate and the developed complications; comparing the results of our study with the results of literature.

Material \& Methods:The study is of a retrospective character and includes all patients with abdominal trauma, presented in the Emergency Department to University Hospital of Trauma, Tirana, from May 2016 to May 2018. The study sample was taken randomly, without any study restriction.

Results:During the two years, 228 patients with liver trauma were reported, of whom 177 (78\%) with blunt liver trauma and 51 (22\%) with penetrating injuries. Men (72\%) were more affected than females (28\%) with a male female ratio of 7: 3 . The most common cause of the injuries was car accident, while the most affected age group was 16-35 years old.
}

In our study patients with blunt Liver trauma $62 \%$ were treated NOM and 38\% OM.

CONCLUSIONS: NOM is the chosen method for the management of blunt liver trauma in hemodynamically stable patients. NOM significantly improves the outcome compared to $\mathrm{OM}$, in terms of decreased abdominal infections, decreased transfusions, and decreased hospitalisation period. However, hemodynamically unstable patients with peritonitis should undergo immediate surgical intervention.

Keywords:liver injuries, injured abdominal, Blunt, penetrating...

$O P-095$

\section{Assessment of Trauma Patient in the Tertiary Centre for Trauma}

\section{Agron DOGJANI}

Author Information:

General Surgeon, University Hospital of

Trauma, Tirana, ALBANIA

\begin{abstract}
Introduction:Trauma can be defined as the organism's reaction to the action of an external agent. Traumatic injury is a leading cause of death globally among persons under the age of 45 . Over 5 million deaths occur each year as a result of injuries, representing $9 \%$ of the world's mortality.

Non-fatal injuries are among the leading global causes of emergency department visits, hospitalizations and long-term morbidity, accounting for a large portion of the burden on health systems.

Initial evaluation and administration of traumatized patients after being brought to a third level trauma centre should be done in a specialized area of an emergency department that is already designed in trauma management. Time of injury is the essence of survival for a life-threatening trauma.

Proper initial care at the scene affects the morbidity and mortality of the traumatized patient.

Prolonged transportation time or inadequate hospital care increases the demand for early restoration of rapid tissue perfusion before the physiological changes of the injured patient reach hospitalization.
\end{abstract}


On the other hand, the lack of recognition of management protocols by trauma management personnel increase morbidity and mortality in this patient group. This presentation addresses the management of traumatized patients as these patients arrive at an emergency department at a hospital or tertiary trauma centre.

Keywords:tertiary trauma centre, traumatized patients, injured patient, life-threatening trauma

$O P-096$

Cost-effectiveness of Thyroid Fine Needle Aspiration Cytology for Diagnosis and Surgical Treatment of Thyroid Malignant Pathologies in Paediatric Age

Ilir ALIMEHMETI ${ }^{1,2,3}$, Myriam HUNINK ${ }^{3,4}$

Author Information:

${ }^{1}$ Projects and International Affairs Unit, Faculty of Medicine, University of Medicine, Tirana, ALBANIA

${ }^{2}$ Department of Family and Occupational Health, Faculty of Medicine, University of Medicine, Tirana, ALBANIA

${ }^{3}$ Department of Epidemiology, Erasmus University Medical Center, Rotterdam, The NETHERLANDS

${ }^{4}$ Department of Health Policy and Management, Harvard School of Public Health, Harvard University, Boston, MA, USA

\footnotetext{
Abstract

Introduction:Thyroid nodules are rare during pediatric age, affecting $1.7 \%$ of children and adolescents, but the high malignancy prevalence at presentation has warranted an aggressive clinical approach. The most frequent surgery technique is total thyroidectomy, which is followed by shortterm and long-term complications. Fine needle aspiration (FNA) cytology is the most accurate tool in the diagnosis of a thyroid nodule. However, there are contradictions if it should be used ad a first-line strategy in the paediatric age. Thus, our aim was to analyze cost-efectiveness of surgery, FNA and observation of thyroid malignant pathologies in paediatric age.

Material and Methods: A decision model was created to trade-off between three different strategies:

Surgery for all nodules, regardless of their malignant or benign nature;
}

Testing through FNA, and then perform surgery according to the FNA result;

Observation, regardless of their malignant or benign nature.

Cost-effectiveness was calculated in terms of costs and quality-adjusted life years (QALY). All variables were entered as distributions. Willingness to pay was set to $50,000 \$$.

Results: In the cost-effectiveness analysis, surgery was dominated by FNA. Observation was less effective and cheaper than FNA, which presented an ICER value of 46,948.48. In the 1-way cost-effectiveness sensitivity analysis for the probability of malignancy from $0 \%$ to $100 \%$, FNA was always superior to the surgery strategy, and the threshold of superiority to the observe strategy was probability of malignancy $=23.5 \%$. In the probabilistic sensitivity analysis, FNA resulted superior to surgery.

Conclusions: FNA is more cost-effective than the surgery strategy in the diagnosis and clinical work-up of thyroid nodules in children.

KEYWORDS: Thyroid nodules, FNA, thyroidectomy, malignant or benign nature.

$O P-097$

Spontaneous Fracture of the left Proximal 1/3 Humerus Shaft in a Female Patient with Multiple Myeloma. A Case reports.

\section{Eriselda TAULLA}

Authors Information:

Military Medical Unit, University Hospital of Trauma, Tirana, ALBANIA

\begin{abstract}
Introduction: Spontaneous fractures are common in patients diagnosed with multiple myeloma and when happens the risk of death in those patients is increased. In this case a 65 years old female diagnosed with Multiple myeloma, developed a spontaneous fracture of the proximal $1 / 3$ humerus shaft while dressing, during the induction therapy.

A 65-year-old caucasian female with a medical history of six month of lumbago, presented to the hospital complaining fatigue, reduced appetite and severe immobilizing bone pain. She reported no other disease. Laboratory findings demonstrated $\mathrm{Hb} 12 \mathrm{~g} / \mathrm{dL}$,
\end{abstract}


calcemia 11,4mg/dl, proteinemia 10,0 g/dL, IGA 2870 mg/dL, Kappa 1920 mg/dL, creatinine $0,6 \mathrm{mg} / \mathrm{dL}$. Bone marrow aspiration showed 56\% plasma cells. MRI showed multiple osteolytic lesions in the scull, ribs, pelvic bones and vertebral compression fractures.

The diagnosis was "Multiple Myeloma IGA Kappa stage II/A". She started the induction therapy with Bortezomib. The day $6^{\text {th }}$, while dressing up, she developed a fracture of the left proximal 1/3 humerus shaft. She was treated with sling immobilization. She is still on therapy with Bortezomib.

Conclusion: All the patients with multiple myeloma should be considered at high risk of mortality during the induction therapy. The fractures during this time worse the prognose. Keywords:Spontaneous fractures, multiple myeloma, humerus, Bortezomib

$O P-098$

\section{Hospital Infections Management}

Esma SHARKA, Natasha MERKO

Author Information:

University Hospital of Trauma, Tirana ALBANIA

\footnotetext{
Abstract

Introduction:Awareness of professionals, relatives, relatives and visitors to Hospital Infections. The infection bites are all hospital facilities that serve to spread the infection, just as medical staff Infectious Infections (IS) or nosocomial infections are circulating and acquired in the hospital During the hospitalization of patients, therefore, receiving another infection plus during hospital stay.

There are no incubation periods either at the incubation time or at the time of laying. Infection begins with the onset of temperature on the third day after laying (48 hours to 72 hours) Nosocomial infection is an indicator of the quality of service! The source of infection is all hospital facilities that serve the spread of infection, as well as medical personnel. For the reduction of morbidity, mortality in hospital beds, IS reduces the quality of life If they are poor and are also very costly High nosocomial infections are indicators of the quality of service
}

Infants receiving hospital attend 2 to $12 \%$ (on average $5 \%$ ) of the patients. IS have mortality of about $1 \%$ and contribute to a $3 \%$ mortality increase in other diseases. Causal pathogens, gram-negative bacilli occupy the bulk of the list of pathogenic germs of IS

Knowing the protective measures for IS reduces the risk of occurring nosocomial infections in the patients and the health worker. Minimizes the rate of nosocomial infection and protects the health personnel and visitors from the risk of infection. These infections are very problematic Because they are very risky around the globe, awareness, implantation of the prisons on the recognition and protection of hospital infections, and risk management, their reporting indeterminacy at the right time of the incidents, the evaluation of the working conditions increase sharply Staff orientation in hospital care by implementing specific guidelines.

Conclusion: From the observation, ISs in hospitals are present and problematic, but that much work and care by staff can be eliminated. More information is available from the staff. Awareness and engagement in all structures for this classroom

Keywords:infection, contamination, tubes, incubation, sterilization

OP - 099

Radioactive Processes in Food Consumer Products, Positive and Negative Effects

\section{Esma SHARKA ${ }^{1}$, Natasha MERKO ${ }^{1}$}

Authors Information:

${ }^{1}$ University Hospital of Trauma, Tirana, ALBANIA

Abstract
Introduction: The importance of evaluating
the effective dose in the human body from
ionizing radiation is wide and quite complex.
It requires ongoing study and evaluation.
Materials and Methods:In this paper we
used the Retrospective study method, which
presents data on the two main contributors to
indoor radiation, the activity of Po-210 in
mussel specimens in the Butrint Lagoon,
studied during spring and autumn and radon 
concentration. Rn-222 indoors in some major cities of Albania.

RESULTS: showed twice as high values of Po-210 activity in mussels during spring compared to activity values found in autumn season, due to different physical and biological factors. These results are comparable to other studies and do not pose any significant risk to public health in Albania. On the other hand, the study presents measurements of radon concentration indoors using the passive method and the work begun to create a radon concentration map in our country. The measurements cover $10 \%$ of the entire territory of the country and will serve to identify Radon Risk Areas in the Republic of Albania for which an action plan will be prepared to reduce Radon concentrations in these areas.

Conclusions: It is important to note that such studies should inform the public about the problems that alpha radiation brings and mainly the high concentration of Radon in the workplace, schools, homes, etc., where we each spend $80 \%$ of our time. .

Keywords:Natural Radioactivity, Po21 0 in mussels, Annual Effective Dose, Radon.

$O P-100$

Pigmentation Diseases, with Negative Impact on the Quality of Life in Individuals Affected.

Ardiana SINANI ${ }^{1}$, Dorela VASHA ${ }^{1}$, Eriselda TAULLAJ $^{1}$, Artan OSMENLLARI ${ }^{1}$, Suzana CAKONI $^{1}$.

Authors Information:

${ }^{1}$ Military Medical Unit, University Hospital of Trauma, Tirana, ALBANIA

\footnotetext{
Abstract

Introduction:Vitiligo is an acquired dermatological pigmentation disorders of the skin. It causes loss of pigment on effected areas of the skin or mucosae and is characterised by milk white, non-scaley lesions with distinct margins.
}

Results:1758 individuals who come in Military Hospital Tirana, Albania in Dermatology Service in period October 2014 - March 2015 was examined having or not Vitiligo. 39 Cases with Vitiligo were diagnosed through the physical standard examination. In these 39patients diagnosis with Vitiligo were applied DLQI questionnaire:

From 39 patient taken in consideration in this research study 22 (56.4\%) were females and 17 (43.6\%) were males, it was noticed that gender was influential in the degree of concern that brings Vitiligo $(p=0.01$,), and in the degree of embarrassed and anxiety due to this skin disease $(p=0.01)$, where females were proven to be the most sensitive category.Resulted that age group was determinant in the skin disease (vitiligo) impact on social activities ( $\mathrm{p}=0.007)$, in the skin disease impact for the selection of clothes $(\mathrm{p}=0.007) \ldots$.

Conclusion:Skin diseases are very common, affecting over a quarter of the population. Vitiligo is not life-threatening skin diseases but have a significant impact on the quality of life of patients and cause considerable psychological distress. Finally, the etiology and pathogenesis of vitiligo remains unclear. It is still not understood what causes the destruction of melanocytes. Also, uncertainties remain about the natural history and epidemiology of this disease. A variety of methods for re-pigmenting the skin of people with vitiligo have been tried in but vitiligo treatment is a major challenge If a cure for vitiligo is to be found, further research is required in understanding the etiology, epidemiology and natural history of vitiligo. Current treatments help to alleviate symptoms for temporary re-pigmentation of vitiliginous patches, but these do not cure the underlying disease.

Keywords:Skin, pigmentation, vitiligo.

$O P-101$

\section{COPD and Lung Cancer, the Coexistence}

Jolanda NIKOLLA ${ }^{1}$, Daniela XHEMALAJ ${ }^{2}$, Hasan HAFIZI ${ }^{2}$

Author Information:

${ }^{1}$ American Hospital 3, Department of Internal Medicine, Pulmonology, Tirana, ALBANIA. 
${ }^{2}$ University Hospital of Lung Diseases "Shefqet Ndroqi" Tirana, ALBANIA.

\begin{abstract}
Introduction:Chronic obstructive pulmonary disease (COPD) is a significant risk factor for lung cancer already well known. Approximately 1\% of COPD patients develop lung cancer every year, which may be associated with genetic susceptibility to cigarette smoke. Chronic inflammation caused by toxic gases can induce COPD and lung cancer. Low-dose computed tomography (LDCT) is an effective procedure for the early detection of lung cancer in high-risk patients.
\end{abstract}

Materials and Methods: We retrospectively reviewed the medical records of patients who were diagnosed as COPD, based on Spirometry and LDCT findings from January 2010 through March 2016 in our hospital and in some of the lung health centers in Tirana. Lung cancer was an incidental finding in them. Chest HRCT images were analyzed by two pulmonologists and radiologists independently. Biopsy examinations were also evaluated by two independent pathologist specialists

Results: In total $18 \quad(\mathrm{~N}=18)$ cases with COPD, 11 (61\%) males and 7 (39\%) females. All patients are current or ex heavy smokers, predominates males. Lung cancer was present in 4 (22\%) COPD male patients. They all had peripheral primary lung cancers and as e result the biopsies was obtained by trans parietal fine needle aspiration procedure. The distribution of the histological types was: 3 cases with squamous cell carcinoma and 1 case with small cell carcinoma. Two patients with lung cancer have died. Their meantime of survival was 2 years. These 4 patients had higher unit pack-years (UPY) smoking values, lower pulmonary functional state and higher values of pulmonary hypertension (HTP) than the other COPD patients.

Conclusions: Lung cancer in patients with COPD patients represents a specific entity with a poor prognosis, that further poses the most characteristic and severe model of tobacco-related diseases. The prognosis of lung cancer patients with COPD is worse than the prognosis of patients without COPD, because of inadequate cancer treatments, and poorer pulmonary function and quality of life.
The most effective lung cancer prevention measure is smoking cessation.

Keywords:Lung cancer, Chronic obstructive pulmonary disease.

$O P-102$

A Serious Self-Injury Attempted Case, following a Neurosurgical Intervention, on a Patient with Epilepsy in Comorbidity with Major Depressive Psychotic Disorder (A Case Report)

Fatbardha MYSLIMAJ ${ }^{1}$, Henri KOLANI ${ }^{2}$, Ilira CAKO $^{1}$, Ermira TOTRAKU ${ }^{1}$

Authors Information:

${ }^{1}$ Psychiatry Service at University Hospital Center

"Mother Theresa", Tirana, ALBANIA

${ }^{2}$ Sugery Department at University Hospital Center

"Mother Theresa", Tirana, ALBANIA

\begin{abstract}
Patient E. H., age 39, presents to Surgical Emergency, with a cut wound: Sinister mammectomy. According to family and patient referrals, the action came as a result of a suicidal attempt. Suffers from Epilepsy since the age of 2 and several years from depression, leading to a suicidal attempt before. She has been treated with antiepileptic drugs for years and underwent neurosurgical intervention in Italy a month ago, which was successful.

The patient shows a regular appearance and according to the chronological age, maintains partial eye contact. Her mimic expresses an apathetic and cold state, without alterations of her mood. Refers that after neurosurgical intervention she has had severe headaches for weeks, pains that did not enable her to sleep or perform normal daily activities. In one of these crises the patient has injured herself, by removing her left breast. Communication is apathetic, cold, distant. Thoughts are negative and worrying about her health. Perception of the environment and reality, cognition and insight damaged. When asked why she chose to damage her breast, refers she had a feeling of emptiness and therefore wanted to remove it. Her headaches were also unbearable and this led her to perform this injury. Through communication the patient provides a lot of information about psychotic elements responsible for the bizarre action.
\end{abstract}


Treatment with antipsychotics, antidepressants, mood stabilizers and anxiolytics, brought an improvement in the psycho-emotional state. Such cases are rarely described in contemporary literature, the consequences of severe psychotic and affective disorders.

$O P-102$

\section{Pain Treatment in Emergency Department}

Hektor SULA

Authors Information:

Faculty of Medicine, University of Medicine Tirana, ALBANIA

\begin{abstract}
Introduction: Acute pain is defined as an unpleasant sensory and emotional experience associated with actual or potential tissue damage as well as activation of neurochemical receptor and mediator responses.
\end{abstract}

Pain is the presenting complaint for up to $70 \%$ of visits to the emergency department (ED). There is a myriad of strategies to treat and diagnose pain. The effective strategies are those with adequate and timely pain relief without adverse effects.

An accurate recognition and assessment of a patient's pain is the central aspect of effective pain management and is essential to any effective analgesic strategy.

Acute pain follows injury and usually resolves as the injury heals. Acute pain may be, but is not always, associated with objective physical signs of autonomic nervous system activity such as tachycardia, hypertension, diaphoresis, mydriasis, and pallor. When the cause of acute pain is uncertain, establishing a diagnosis is the priority of the emergency physician. Symptomatic treatment of pain should be initiated while the diagnostic evaluation is proceeding. In general, it is inappropriate to delay analgesic use until a diagnosis has been made.

The treatment of acute and chronic pain is different, and confusion between the two leads to poor management of patients. Acute pain should be approached with the intention of providing relief to a limited degree, individualized to each patient, with a plan to taper medications as symptoms improve. Chronic pain assumes a baseline level of pain that is best treated with a consistent approach to minimize baseline discomfort and minimize the adverse effects of both pain and pain treatment on the patient's lifestyle.

Optimal acute pain management is patient specific and pain syndrome targeted when feasible, using a multimodal approach that includes pharmacologic and nonpharmacologic interventions. Base the assessment of pain and need for therapy on an overall accounting of patient status, including functional assessment, rather than solely on patient-reported pain scores.

Conclusion: Pain is the most common complaint in the ED. Having a consistent, integrated, and well-planned approach will optimize the experience for patients as well as medical providers

Keywords:Pain, emergency, treatment.

$O P-104$

\section{The Obese (Emergency-) Patient: Business as Usual?}

\section{MarkusKONERT}

Authors Information:

1 Anestesi Sevices at RoMed-Klinik Bad Aibling, GERMANY

\begin{abstract}
The prevalence of significant obesity continues to rise in both developed and developing countries and is associated with an increased incidence of a wide spectrum of medical and surgical pathologies. Thus, experienced anaesthetic and surgical staff should manage obese patients.

The variety of comorbidities and the pathophysiological changes of the respiratory and cardiovascular system lead to a significantly increased risk of complications. To master these risks, special processes and treatment management are necessary, in which knowledge of (patho-) physiological alteration of the obese is essential.
\end{abstract}

Obese patients require specially trained hospital and rescue personnel, suitable equipment and adequate structural equipment, which should be provided by all hospitals and health care institutions. 
In my talk I would like to share my experience in the treatment of obese patients from an anesthesiologist's point of view.

$O P-105$

\section{Trauma in Pregnant Women}

Rudin DOMI ${ }^{1}$, Gentian HUTI ${ }^{1}$, Asead ABDYLI ${ }^{1}$

Authors Information:

${ }^{1}$ Department of Anesthesia and Intensive Care Medicine, American Hospital 3, Tirana, ALBANIA

\begin{abstract}
Introduction: Trauma in pregnancy is a great concern in modern era. The pregnant women make more challenging the anesthetic and ICU care due to both organisms the women and the fetus.
\end{abstract}

Materials and Methods:Regarding epidemiology of trauma in pregnancy, several data come from policy, from national services of different states, and some of them are also reported in literature as well. It is recently reported that the incidence is evaluated $6-7 \%$, and car crush is the major reason over passing physical assaults and falling. This kind of trauma is also the major cause of mother non obstetrical death.

Results:Pregnant women have some physiologically features. Pregnancy related cardiovascular changes are increased cardiac output, vasodilatation, increased heart rate, increased blood volume, decreased blood cells concentration, and decreased central venous pressure. These changes can mask the severity of hypovolemia, the severity of shock states, and can mimic myocardial ischemia. Respiratory changes include reduced functional residual capacity due to weight gain, increased respiratory rate and volume in minute. These changes can induce increased oxygen consumption and can lead to rapid desaturation. The pregnant woman can be presented with full stomach, decreased sphincter tone and increased risk to aspirate, may have difficulties in ventilation and intubation, and may have airway edema too.

Conclusions:To conclude we must be focus on mother condition because improving mother conditions can improve baby conditions respecting trauma guidelines as well. A multidimensional team must be always available in order to improve the prognosis.

KeyWords:trauma, pregnancy, fetus.

$O P-106$

\section{Treatment of Traumatic Hemorrhagic Shock in Intensive Medicine}

Nehat BAFTIU ${ }^{1}$, R.BAFTIU1

Authors Information:

${ }^{1}$ University Clinical Center of Kosovo - Pristina, KOSOVO

\begin{abstract}
INTRODUCTION: The shock represents a state of inadequate tissue perfusion as a result of circulatory insufficiency. Hemorrhage is the most common cause of shock in injured patients. The goals of this paper are as follows: Analysis of the epidemiological characteristics of traumatic - hemorrhagic shock in Kosovo, for the period July 2016 December 2018.

Material and Methods: As material we used the stories of patients with traumatichemorrhagic shock, treated in the Intensive Care Unit of the University Clinical Center of Kosovo, during the period July 2016 December 2018. The sample consisted of 155 patients treated during the specified period, with verified diagnosis of traumatichemorrhagic shock.
\end{abstract}

Results: We established the study to demonstrate the efficacy of therapy in the hemodynamic stabilization of traumatized, shocked patients in the first 12 hours of intensive care treatment. We categorized the patients according to the classes of hemorrhagic shock. The classification was performed according to the criteria set by the American College of Surgeons. Parameters were assessed: respiratory rate, respiratory load, systolic blood pressure (TAS), capillary refill and Glasgow Coma Scale (GCS). 
Conclusions: Traumatic-hemorrhagic shock is a life-threatening emergency due to the development of multiple organ dysfunction syndrome, which may be due to symptomatology and changes in vital parameters.

Keywords: Hemorrhage, shock, injured patients,

OP -107

Bilateral Pulmonary Embolism after Hip Surgery: A Case Report

Albert LLESHI $^{1 *}$, Ilir HASANI ${ }^{1}$, Ana STOJKOVSKA $^{1}$, Bijana POPOSKA ${ }^{1}$, Angela TRPOSKA $^{1}$, Filip NAUMOSKI ${ }^{1}$, Andzela VITANOVA $^{1}$

Authors Information:

${ }^{1}$ University Clinic for Traumatology,

Orthopaedic Diseases, Anaesthesia,

Reanimation, Intensive Care and Emergency

Centre in Skopje, RN of MACEDONIA.

\begin{abstract}
Introduction: Pulmonary embolism (PE) ranges from incidental, clinically unimportant occurrences to causing sudden death. Virchow's triad of local trauma to the vessel wall, hypercoagulability and stasis of blood leads to thrombus formation in the veins of the legs which may dislodge and embolize to the pulmonary arteries with potentially serious consequences (1). Prompt diagnosis and treatment reduces the risk of complications and death.

Pulmonary embolisms is a potentially lifethreatening condition where a dislodged thrombi most commonly from the legs travels to the lungs and blocks one of the pulmonary artheries. The combination of the nonspecific symptoms, patient medical history and the risk factors should stear us into taking in consideration the pulmonary embolismus diagnose.

Heparin constitutes the cornerstone of management of PE. In this report we present a case od pulmonaryembolisms in a 70-yearold female that occurred three weeks after hip surgery, was presented to the emergency department with chest pain and shortness of breath. Her vital signs were as follows: heart rate 80 beats/minute, respiratory rate 18
\end{abstract}

breathes/minute, blood pressure 120/80 mmHg and oxygen saturation $86 \%$. Three weeks earlier she had been operated on for a right-sided hip fracture and had received low molecular weight heparin prophylaxis postoperatively...

Conclusion; Pulmonary embolism is a lifethreatening condition that should be prompty recognized. Surgical patients are at increased risk of developing a PE, especially after orthopaedic hip and knee surgery, because of positioning during surgery and mainly immobility after surgery, that contributes to a increase venous stasis.

Keywords: pulmonary embolism, activated clotting time,heparin.

$O P-108$

\section{Trauma and Coagulopathy}

Asead ABDYLI 1, Rudin DOMI 1, Gentian HUTI ${ }^{1}$

Authors Information:

${ }^{1}$ Department Anesthesia and Intensive Care, American Hospital 3, Tirana, ALBANIA

\begin{abstract}
Introduction: Hemorrhagic shock from blood loss is a major cause of mortality in trauma patients. Bleeding is due to an intrinsic dysregulation of the blood coagulation known as trauma-induced coagulopathy (TIC). TIC is faced in the presence of both hypoperfusion from blood loss and tissue injury.
\end{abstract}

Materials and Methods: We review the literature, in order to highlight the modern concepts and mechanisms contributing to TIC. Understanding these mechanisms can improve treatments strategies, morbidity, and mortality of severe traumatized patients.

Results: The mechanisms of coagulopathy in trauma patients have been explained by close examination of the biochemical and cellular changes due to tissue injury and hemorrhagic shock. Mechanisms contributing to TIC include anticoagulation, consumption, platelet dysfunction, and hyperfibrinolysis.

Conclusions: The anesthesiologist and intensivist often are faced with traumatized patients. The surgical team must be prepared to promptly treat trauma induced 
coagulopathy, and good knowledge about this complication is the first step.

Keywords: Hemorrhagic shock, trauma, coagulopathy.

OP- 109

The fascia Iliaca Compartment Block in the Emergency Department

\section{Markus KONERT ${ }^{1}$}

\section{Authors Information:}

${ }^{1}$ Anestesi Sevices at RoMed-Klinik Bad Aibling, GERMANY

\begin{abstract}
A regional nerve block with long acting local anaesthetic can provide effective analgesia for patients with fractured neck of femur and may reduce the need for opiate analgesia.

The Fascia Iliaca Compartment block is as effective as a 3:1 block targeting the femoral nerve, obturator nerve and lateral cutaneous nerve, and can be achieved easily using ultrasound guidance.

As the site of the local anaesthetic injection is distant from the neurovascular bundle it has a low complication rate.

We did cadaver studies with dyed water to gain information about the ideal puncture site and to visualize the spread of the local anesthetic along the fascia.
\end{abstract}

KEYWORDS: Fasca iliaca, block, emergency department.

$O P-110$

Post Factum Evaluation of Radius Fractures in the Typical Place in Relation to Age, Gender, Osteoporosis Rate, and Treatment Outcome.

Elona HASALLA ${ }^{1}$, Ilda TAKA ${ }^{1}$, Ilia MAZNIKU1', Blerta HASALLA ${ }^{1}$, Sulejman BAHA ${ }^{2}$, Elona DYBELI ${ }^{1}$.

Authors Information:

${ }^{1}$ Lecturer in Nursing Department, Faculty of Medical Technical Sciences, University of "Aleksander Xhuvani" Elbasan, ALBANIA.

${ }^{2}$ University Hospital Center "Mother Theresa", Tirana, ALBANIA

Abstract

Introduction: To evaluate the influence of the above fractional on the frequency of radius fractures in the typical place, particularly of osteoporosis as the main cause of these fractures at the age of three.

Functional treatment results.

Materials and Methods:Actually 82 patients with radial fracture in the typical place of conservative treatment, implantation and implantation in gypsum, as well as the respective reactive rehabilitation procedures were studied. In the material, they have taken a survey; the age of the patients, the sex, the rate of osteoporosis, the technique of reproduction, how long after the fracture and eventually the results achieved. They were 76 women or $92.6 \%$ and 6 males or $7.4 \%$. The maximum age of 86 years of age, the average age of 75 and the minimum age of 63 In the study, primary is the rate of osteoporosis as potential potential for radius fractures in the typical place.

Results:In our study series, the largest number of patients was female, eighty-two cases or $92.6 \%$ of the study group, the rest of $7.6 \%$ or 6 cases were mammal. The study period was 2 years of 2016-2017 all fractures are in traumatic terrain. Traumatic trauma in $60 \%$ of cases has been overturned or otherwise reported as a direct trauma, whereas in $40 \%$ of cases there has been an indirect trauma. pain, loss of function, different hematoma rates as well as radiological changes were the signs of these injuries. Treatment endpoints were favorable especially when the implantation period of three weeks was respected in gypsum and the beginning of rehabilitation exercises

CONCLUSION:Typical site radius fractures are heavily influenced by regional osteoporosis, age of patients, osteoporosis rate. Generally, benign fractures tend to consolidate. A three-week period is sufficient for the attachment of bone fragments and the origin of rehabilitation. Over-term complications, such as dysfunctional bone atrophy, are more difficult to treat than fractures themselves.

KEYWORDS: Radius, osteoporosis, fracture, rehabilitation.

$O P-111$

Ambulatory Treatment of Upper Limb Trauma and Nurse Engagement 
Elona HASALLA ${ }^{1}$, Ilda TAKA ${ }^{1}$, Ilia MAZNIKU

${ }^{1}$, Blerta HASALLA ${ }^{1}$, Sulejman BAHA ${ }^{2}$, Elona DYBELI ${ }^{1}$.

Authors Information:

${ }^{1}$ Lecturer in Nursing Department, Faculty of Medical Technical Sciences, University of

"Aleksander Xhuvani" Elbasan, Albania.

${ }^{2}$ University Hospital Center "Mother

Theresa”, Tirana, ALBANIA

\begin{abstract}
INTRODUCTION: Outpatient treatment of locomotor injuries in general and upper extremity trauma in particular represents a medical, economic, social and scientific interest. Upper extremity traumas are injuries that are more and more common nowadays and are still a serious concern for individuals who suffer, their loved ones, and society.

Materials and Methods:Our study included 92 traumatized individuals, of whom 60 are male and 32 are female. 56 persons or $61 \%$ were from Tirana and 36 - 39\% were from districts. The study covers the period January 2018 to the end of May 2019.
\end{abstract}

Results:Males were 60 individuals or $65.2 \%$ of the whole series, while females occupied 32 individuals or $34.8 \%$ of the whole series or just over $1 / 4$ of all affected. The age most affected by traumatic injuries in our study is up to in 40 year olds, who make up 59\% of cases.

Conclusions:

1- Upper partial injuries cause significant functional loss and occupy an important place in the case of traumatic injuries.

2-It is important to diagnose and treat these injuries in a timely manner.

3. The provision of first aid at the scene is of the utmost importance and plays an important role in the further treatment of this pathology.

Keywords: trauma, upper extremity,day surgery, ambulatory treatment.

$O P-112$

\section{Sports Trauma and Their Management}

\section{Dorian META}

Author Information:

University of Modena and Reggio Emilia, ITALY. Assistant Professor at Faculty of Technical Medical Sciences, Tirana, ALBANIA.
Specialist in forensic medicine and clinical physiology.Physiotherapist at FIZIO-TRAUMA, Tirana, ALBANIA.

\begin{abstract}
Introduction:Frequently the problems of patients / sportsmen who have a lack of interpretation of information are collected especially in the training area, where the information is related to the systems used. One major problem in the Sports Trauma aspect covers physiotherapists member sports, in real time, determine an immediate diagnosis after an emergency or observation of the traumatic event.
\end{abstract}

RESULTS: $60 \%$ of patients with immediate post-traumatic stress disorder recovered immediately after trauma or long-term hospitalization. In patients who were not treated at around $40 \%$, it was found to be an emergency rate of recovery rapidly following ophthalmic surgery, as well as an emergency application in the emergency room.

CONCLUSIONS: Physiotherapists are required by the specialist to diagnose all types of sports trauma and the way that the patient is presented during an activity or during a probationary traumatic event, the knowledge of which has a direct impact on recovery and recovery. Eighty-three trained to manage problems related to traumatic events and posttraumatic events.

Keywords:Sports trauma, training area, physiotherapist, management.

$O P-113$

Rehabilitation for Patients Following ACL Reconstruction

Anxhela AHMATAJ ${ }^{1}$, Viktor MARKU ${ }^{2}$

Authors Information

${ }^{1}$ Physiotherapist, San Vitale Clinic, Tirana, Albania.

${ }^{2}$ Orthopeadic Surgeon, University Hospital of Trauma, Tirana, Albania.

\begin{abstract}
In our clinic, "San Vitale", patients are routinely referred for physiotherapy after ACL reconstruction.

However, to date the role and amount of supervised physiotherapy required has not been clearly established.We follow a model,
\end{abstract}


which has been developed by clinical observation, patient interaction, and by analyzing outcome measures derived from prospective follow-up patients.

The best outcome scores occurred in patients with combined program: ROM + Strength, a group who attended physiotherapy regularly after ACL reconstruction, comparison with those who attended only infrequently.

$100 \%$ of patients achieved normal knee extension and $97 \%$ of patients achieved normal knee flexion.

Keywords:ACL, reconstruction, rehabilitation

$O P-114$

\section{Review Role of Rehabilitation in Neural Plasticity}

Andromeda KECI ${ }^{1 *}$, Klejda TANI, Joana

XHEMA

Authors Information:

Physiotherapy Department, Faculty of Technical Medical Sciences, University of Medicine Tirana, ALBANIA

\section{Abstract}

Introduction: Verifying if physical therapy, neurostimulation techniques, aerobic fitness and video games can induce neural plasticity making it possible for cortical reorganisation, motor recovery in patients, improvement of cognitive functions and transfer of spatial knowledge in everyday living environment.

Methods:There have been revised scientific articles respectively focused in the role of pain, the role of physical therapy, neurostimulation techniques and video games in cortical reorganization. Articles related to the role of pain have taken in the study subjects with pain, to obsverve its role in cortical reorganization. Studies related to physical therapy and neurostimulation techniques after cerebrovascular accident consisted on the involvement ofthese subjects which exposed to different neurostimulations. Also, related to cogniniton and video games subjects exposed to these interventions for cognitive benefits.
Results: From all articles reviewed there have been effective results ofneurostimulation techniques, aerobic fitness and video games in cortical reorganization inducing neural plasticity toward motor recovery, improvement of executive functions and transfer of spatial knowledge.

Conclusion:Rehabilitation through locomotor training and neurostimulation techniques, improves mobility in subjects after a cerebrovascular accident due to cortical reorganization. Also, through aerobic fitness and video games there have been improvements of cognitive functions. This way, rehabilitation dedicated to the promotion of well-being and health urges beneficial neuroplastic changes in brain corresponding in functional improvement.

Keywords: Cortical reorganization, neural plasticity, aerobic fitness, video games, rehabilitation.

$O P-115$

This abstract has been withdrawn.

$O P-116$

Case Study, Impact and Efficacy of Physiotherapy in Anterior Gleno-Humeral Dislocation on the Geriatric Age.

Selda VEIZAJ $^{1 *}$, Endrit MONE $^{2,}$ Erisa MANE $^{2}$, Aida LAKO ${ }^{1}$

Authors Information:

${ }^{1}$ University "Alexander Moisiu" Faculty of Professional Studies, Durres, ALBANIA

${ }^{2}$ University of Medicine, Faculty of Technical Medical Sciences, Tirana, ALBANIA

\begin{abstract}
Background;Dislocation anterior of glenohumeral joint is when the head of the humerus is out of the shoulder jointdue to direct or indirect forces. This is the most frequent dislotaion, among all human joints. About $1.7 \%$ of people have anterior glenohumeral dislocation during their lifetime.PurposeEvaluation of the efficacy of
\end{abstract}


physiotherapy in the treatment of anterior gleno-humeral dislotacion ingeriatric patients. Material and Methods.Case study, a patient 82-years old treated at University Hospital of Trauma Where initially the Orthopedic doctor has restored the head of the humerus into the shoulder joint through the Kocher method, it's recommended a tutor for a month. The patient underwent physiotherapy for 2 months.

The following tests were used: VAS, Apprehension Test, Relocation Test, Anterior Instability Test.

Results;Based on physiotherapeutic treatment that included 3 stages of treatment 1, 4 and 8 weeks. VAS rate at the beginning of treatment the patient reported vas values = $7-8$; in week four Vas values were reported $=$ $4-5$; and in week 8 vas $=3$. While based on Apley's Scratch test results unable to reach the physician- defined targets that meant poor mobility of the upper limb. In the second phase of rehabilitation this test results in difficulty performing the function of flexion / extension; internal / external rotation or abduction and adduction. At week 8 the patient was able to perform all required movements.

Conclusions:By physiotherapy we achieved the stabilization of articular ROM, improved muscular strength andmobility, improved neuro-muscular control, and patient preparation for functional recovery in activities ofdaily living.

Keywords; Physiotherapy, Dislocation anterior of gleno-humeral, Apley's Scratch Test

$$
O P-117
$$

Radiation protection in Diagnostic Radiology

Bleona SEJDINI $^{1^{*}}$, Ina UZUNI ${ }^{2}$

Authors Information:

${ }^{1}$ Student at Medical University of Tirana, ALBANIA

\section{Abstract}

The aim is to emphasize the importance of radiation protection in medicine, to explain radiation protection protocols in Diagnostic Radiology, focused in occupational exposure, medical exposure and public exposure.
Introduction;Diagnostic Radiology includes general radiography, CT, CBCT, mammography, tomosynthesis, dental radiography (intraoral, panoramic and CBCT), bone densitometry (DXA) and fluoroscopy. All these diagnostic procedures use ionizing radiation (radiation that interacts with matter) - X-rays. Latest reports suggest that there are made about 4 billion X-ray examinations per year worldwide. Does the increasing demand for X-ray imaging procedures has implications for radiation protection of medical staff? The increase in uses of these procedures presents a challenge to ensure appropriate radiation protection of medical staff, patients and public members.

Material and Methods; World literature, practice and work experience.

Results; Radiation protection in medicine is important and unique for a number of reasons. Medical uses of radiation represent more than $99.9 \%$ of man-made radiation exposure and there are billions of these procedures occurring annually in all regions of the globe. Medical uses also result in the largest number of occupationally exposed workers and represent the largest occupational collective dose. This is the main reason why health professionals should be known with radiation protection and safety in medical uses of radiation, including roles and responsibilities, education, training, qualification and competence, and the management system for protection and safety.

Conclusions; Medical uses of ionizing radiation involve a number of health professionals performing radiological procedures such as diagnostic examinations.

Keywords; X-rays, radiation protection, ALARA principle, medical exposure, dose limits, shielding design, quality assurance.

$O P-118$

Scoring Tool for Massive Transfusion in Abdominal Trauma

Ayman EL-MENYAR,

Author Information:

${ }^{1}$ Chef of Trauma and Vascular Surgery, Hamad General Hospital, Doha, QATAR. 


\begin{abstract}
Shock index (SI), is considered as a rapid predictable indicator of hemodynamic instability in trauma patients. Herein, we sought to assess SI in patients with abdominal trauma.

We conducted a retrospective analysis for trauma patients between 2014 and 2017 in a Level 1 Trauma center.

Patients were classified into group I $(\mathrm{SI}<0.8)$ and group II ( $\mathrm{SI} \geq 0.8$ ). Out of 1199 patients with abdominal trauma requiring hospital admission, $49 \%$ had $\mathrm{SI} \geq 0.8,40 \%$ received blood transfusion, $14 \%$ received massive blood transfusion (MTP), and $27 \%$ had exploratory laparotomy. In comparison to lower SI group, patients with $\mathrm{SI} \geq 0.8$ were significantly younger ( $28 \pm 14$ vs. $34 \pm 13$ ), had greater ISS ( $22 \pm 13$ vs. $15 \pm 10)$, and had more positive FAST ( 36 vs $24 \%$ ). $\mathrm{SI} \geq 0.8$ group had more retroperitoneal hematomas (6.0 vs $2.9 \%$ ), splenic injury (31 vs. $25 \%$ ), more blood transfusion (58 vs $23 \%$ ), MTP (25.8 vs $3.1 \%)$, exploratory laparotomy (32.6 vs $22.2 \%$ ), splenectomy (8.4 vs $2.8 \%$ ), longer hospital stay, and higher mortality (10.8 vs. $2.6 \%), \mathrm{p}=0.001$.
\end{abstract}

Liver and bowel injuries were comparable between the 2 groups. SI was significantly correlated with ISS ( $\mathrm{r}=0.35)$ and GCS ( $\mathrm{r}=-$ 0.314). After adjusting for age, sex, ISS, GCS, and abdomen AIS, high SI was independent predictor of exploratory laparotomy (aOR 2.25, $\mathrm{p}=0.001$ ) and MTP (aOR 8.07, $\mathrm{p}=0.001$ ). ROCs and Area under the curve (AUC) showed that SI $\geq 0.80$ predicted the need for MTP (AUC $=0.83$ ) with $85 \%$ specificity, $65 \%$ sensitivity, $28 \%$ PPV and $96.7 \%$ NPV.

Shock index $\geq 0.80$ on admission is associated with worse outcomes and can predict the need for MTP and exploratory laparotomy in abdominal trauma.

$O P-119$

\section{Endovascular Management in Abdominal Trauma}

\section{Hassan AL-THANI}

Author Information:

Senior Consultant, Chef of Trauma and Vascular Surgery, Hamad General Hospital, Doha, QATAR

\begin{abstract}
Trauma continues to be the leading cause of mortality in young population. Truncal hemorrhage is associated with high prehospital fatalities in comparison to fatality rate in the extremity injuries due to the difficulty in controlling the bleed. The role of endovascular evolved rapidly in resuscitation, diagnosis and management stages of trauma patients. The endovascular management seems specifically promising in abdominal injuries, especially in the setting of others concomitant injury. In the resuscitation phase the Introduction of Resuscitative Endovascular Balloon Occlusion of the Aorta (REBOA) is an alternative to open resuscitative thoracotomy with aortic cross clamping to support the circulation until definitive interventions. The availability of hybrid operating rooms with angiographic radiology capabilities offers opportunities for the diagnostic and management of hemodynamic unstable patients by either temporary control of the devastating bleeding using endovascular balloon or with embolization and stenting. This presentation reviews current literature and our experience with regard to the endovascular management in abdominal trauma.
\end{abstract}

$O P-120$

\section{Solid organ injuries}

\section{Hassan AL-THANI}

Author Information:

Senior Consultant, Chef of Trauma and Vascular Surgery, Hamad General Hospital, Doha, QATAR

\begin{abstract}
Solid organ injury includes the liver, Spleen, kidneys and pancreas in isolation or combination. They are known to be highly vascular and thus represent a major source of bleeding post trauma especially with blunt mechanisms. This fact makes them important determinants of mortality and morbidity and a challenge for care giver facing significant poly-trauma in trauma centers regardless of age or gender. The management is multidisciplinary.
\end{abstract}


Those who are hemodynamically unstable with positive FAST will have an immediate exploration laparotomy to control the bleeding accordingly; some cases need a damage control approach in resuscitation and operatively and often a postoperative CT scan and angio-embolization if suspected ongoing bleeding, pseudo-aneurysm or AV fistula is identified before return to OR for definitive completion and fascial closure.

On the other hand Non Operative Management (NOM) is recommended treatment for those who are hemodynamically stable or respond to initial resuscitation and that represent the standard. The advantages decrease LOS, blood transfusion. NOM take place in ICU with close observation clinically, laboratory and imaging repetition within 48-72h with or without the addition of angio-embolization which help to increase the success of NOM and control early posttraumatic pseudo-aneurysm development eliminating risk for delayed bleeding by blocking bleeding damaged vessels. Failure rates are variable for different organs; renal (0-10\%), Hepatic (1-15\%), Splenic (2-52\%).

Important management controversies remain where to admit, when to determine failure, role of angio-embolization, how long should you keep those patients? Timing for thromboprophylaxis, post-splenectomy vaccination timing, vaccination after angio-embolization, follow up and needed rest.

$O P-121$

\section{Revisiting Open abdomen}

Husham ABDELRAHMAN, MD, FRCSI, CABS

Author Information:

Senior Consultant Trauma Surgery, Director of Trauma Resuscitation Unit,

Hamad trauma center. Hamad General Hospital, Hamad Medical Corporation, Doha, QATAR

\footnotetext{
Abstract

Open Abdomen remains a life-saving procedure in selected patients and a potential source of morbidity and mortality. Data suggest that it is over-utilized and there is generally low compliance to published guidelines. It is main use in critically injured patients with massive bleeding often
}

abdominal and ongoing massive transfusion and to a greater less use in acute care for septic abdomen with septic shock, mesenteric ischemia and acute severe pancreatitis. Nowadays, it is used rarely for controlling abdominal compartment syndrome not responding to aggressive medical treatment. Advances in resuscitation have impacted the frequency of utilizing this technique and some novel interventions like direct peritoneal resuscitation have impacted also it is use and the expedite closure course as well as advanced open abdomen dressing techniques.

Those cases are prone to a multitude of local and systemic complications both in short and long term and thus it is demanding condition with high associated morbidity (30\%), cost and mortality (ranged 11-39\%).

The concept of total management of opened abdomen is "key", and represents a multidisciplinary team of highly motivated health professionals to enhance and optimize the management of these vulnerable and sick patients with open abdomen in a global and inclusive model of care. Well-designed protocols and prospective research trials is in high demand to further clarify the problem and inform clinician decision making and best practices.

$$
O P-122
$$

\section{The Challenges in Diagnosis and} Management of Blunt Bowel Injury

\author{
Ismael Yousif Mahmood ALOMAR
}

Author Information:

Associate Consultant, Trauma Surgery, HMC, Assistant Professor, Weill Cornell Medicine -QATAR

\begin{abstract}
Blunt abdominal trauma causing bowel injuries are uncommon and found in about $1 \%$ of patients undergoing CT evaluation. Clinical signs of bowel injuries such as abdominal tenderness, rigidity, absent bowel tones are present in less than $50 \%$ of patients. Clinical evaluation is more difficult in polytrauma patients especially those with
\end{abstract}


head and spinal cord injuries. An absence of the specified CT findings was encountered in $9.1 \%$ with surgically proven bowel injuries. The presence of free fluid in the abdomen without solid organ injuries: Mesenteric tear which may or may not require repair, missed solid organ injury. Fluid leaking from retroperitoneal hematoma associated with a pelvic fracture. Postoperative complication of intestinal anastomosis after blunt abdominal trauma. We reviewed all intestinal injuries in adult patients who underwent laparotomy for blunt abdominal trauma between January 2008 and December 2015.One hundred sixty patients with intestine injuries were identified. The overall incidence of abdominal complication was $17.5 \%$. Wound infection $10 \%$. Intra-abdominal abscess $8.1 \%$. Anastomotic leak 3.8\%. There were two deaths occurred because of the complication from the bowel injury. Based on finding, it can be argued that patients, who have blunt small or large bowel injuries, even if operated early, have significant morbidity and mortality. Blood transfusion, high lactate level and number of re-laparotomies were found to be significantly related to intraabdominal complication.

$O P-123$

\section{Cast Treatment of Achilles Tendon Tear After Surgical Repair. Cast Technician Perspective.}

Arben GJONEJ'1 , Risida GJONEJ' ${ }^{1}$, Astrit PEKA ${ }^{1}$, Edvin SELMANI ${ }^{1}$, Vilson RUCI ${ }^{1}$

Authors Information:

${ }^{1}$ Service of Orthopedic, University Hospital of Trauma. Tirana, ALBANIA.

\section{Abstract}

Introduction: The aim of this study is to show the results of cast treatment of Achilles tendon tear patients treated surgically during 2017-2018 from the cast technician's perspective.

Materials and Methods: We studied all the cases with complete tear of Achilles tendon treated surgically in our Institution during period of January 2015 until December 2016.All data were collected and analyzed according to package SPSS version 20. Frequencies and percentages are used to evaluate variables. We considered significant p values less than 0.05 .

Results:During 2017-2018 there are 77 cases with complete tear of Achilles tendon treated in our Institution. 7 Cases (9.1\%) were females and the age group were 25-35 years old. 5 Cases out of 9 females were right side and 2 were left side. 70 cases $(90.9 \%)$ were males and out of these 7 cases (10\%) were in the age group 25-35 years old and 50 patients (71.4\%) are in the group age 35-45 years old and 13 patients were above 45 -year-old. From these patients 55 patients were tendon ruptures of the right side and 15 were left side. All patients were treated surgically first and then with cast. 70 cases were acute tears and 7 cases were chronic tears with the later having prolonged rehabilitation time. Correlation of variables of initial time of treatment and rehabilitation is significant $\mathrm{p}<0.05$.

Discussion:Complete tear of Achilles tendon patients reported in our Trauma center treated surgically followed by cast treatment have been 77 cases during period of 20172018.Male gender has dominance. Type of treatment is surgical repair of tendon. Time of surgery is crucial in rehabilitation outcomes. Preferred treatment should be surgical repair followed by cast immobilization.

Keywords: Achilles tendon tear, cast immobilization.

$O P-124$

\section{Urinary Tract Infections in Women During Hospitalization}

\section{Afërdita ADEMI}

Author Information:

Medical Faculty - University of Tetovo, RN of MACEDONIA.

Department of Urology, Clinical Hospital Tetovo, RN of MACEDONIA.

\footnotetext{
Abstract

Introduction:A urinary tract infection (UTI) is caused by different types of bacteria which multiplying in urine. When the infection affects the lower part of urinary tract it is
} 
known as a cystitis or bladder infection. Nurses in urology department must be able to treat uncomplicated low urinary infection in women.

Material and Methods:in this retrospective study are included 125 women treated in our department during a period between January 2017 - 2019, which were catheterized during hospitalization. Catheterization was performed with siliconized Foley catheters while during catheterization we followed all antiseptic and disinfection rules.

Results:in 125 women aged between 20 - 70 years old hospitalized in our department in a period between June 2017-2019, the risk of urinary tract infections in the first three days was very low, respectively, only in one of the patients $(0.8 \%)$ contamination of the urinary tract with bacteria was recorded. With the extension of catheterization time, the risk of developing urinary tract infections increased progressively, so one week after catheterization the lower urinary tract infection was recorded in seven women (5.6\%), whereas teen days after catheterization in eleven patients was registered the presence of urinary tract infection. Most of the patients with urinary tract infections were over thirty-five years old. Urine culture showed that the most common cause of urinary infections was E. coli, while the patients were treated with more frequent replacement or sometimes with complete removal of the urinary catheter and the administration of antibiotics according to the antibiogram results.

Conclusion:in patients hospitalized in the urology department the most common risk factor for the development of lower urinary tract infection are duration of catheterization, age and female sex. Nurses play an important role in early detection and follow-up of patients with urinary tract infections.

Keywords: low UTI, women, nurses.

$O P-125$

\section{Premature Birth and the Contribution of the Midwives}

Risida GJONEJ ${ }^{1}$, AlbanaPOLOSKA ${ }^{1}$, Etleva SMAKAJ $^{1}$, Valbona BEZHANI ${ }^{1,}$ Flora ZYBERAJ $^{1}$

Authors Information:
${ }^{1}$ Lecture, Faculty of Medical Technical Science, University of Medicine of Tirana, ALBANIA.

\begin{abstract}
Introduction:According WHO, a birth is premature when it happens before 37 gestational weeks calculated from the first day of the last menstrual period. Risk factors that affect in this pathology are classified in two groups: 1) spontaneous, 2) fetal/maternal reasons or both.
\end{abstract}

Materials and Methods: This is a retrospective study. The information is collected from the clinical charts for the period January 2018 till December 2018. Demographic data of the mother were also taken into consideration (which are expressed in frequency and percentage) and how they affect premature birth as well as the risk factors how they affect premature birth too. Data processing was performed by SPSS 20.0 package. We used chi- square test for the categorical variables and the p value 0.05 .

Results:For the period January 2018 till December 2018 have born 6620 babies of which 515 (7.7\%) are premature births. The age group of mothers are: 17-24 years old $35.7 \%$, 25-30 years old 26.3\%, 30-35 years old $22 \%$ and over 35 years old $16 \%$. The correlation between the age variable and the diagnoses is significant and the $\mathrm{p}$ value is 0.01 . The rate of the mother which deliver for the first time (primipare) was 292 cases (56.7\%) and pluripare 223 cases (43.3\%). The most dominant influencing factor was high blood pressure with 241 cases (46.8\%). The correlation between diagnoses and risk factors variables was significant with $\mathrm{p}$ value 0.001 . $78 \%$ of the mothers delivered by caesariansection. 139 patients (27\%) used tocolytics.

Conclusions:From 6620 babies that have born, 515 of which are premature births. The most dominant influencing factor in this diagnose was high blood pressure and $78 \%$ of the babies have deliverd by cesarean section. The young age of the mother but also the age of 35 and over affects the premature birth. 
The low use of tocolytics are due to the low cases of membrane premature ruptures. Midwives' contribution is seen in the proper care and implementation of the tocolytics described. The midwife helps the mother identify early signs of premature birth activity as well as helps her identify and minimize risk factors.

Keywords:Premature birth, tocolytics, caesarian section.

$O P-126$

Post-traumatic stress syndrome, in hospital ward settings

Natasha MERKO ${ }^{1}$, Hasije DURAKU1 ${ }^{1}$, Etlleva RAMALIU 1

Authors Information:

${ }^{1}$ Nurse Service University Hospital of

Trauma, Tirana, ALBANIA.

\begin{abstract}
Introduction:This Reference discusses recent findings from epidemiologic surveys of traumatic events and posttraumatic stress disorder (PTSD) in the workplace, including prevalence, risk factors and consequences, risk associated with specific traumatic events, and on morbidity and comorbidities associated with these events.
\end{abstract}

Materials and Methods:We have had a descriptive study in voters, presentation of 100 Nurses. On tire exams you score points for exposure, PTSD, Look in survey sheets calling the terms 'trauma' and 'post traumatic stress disorder' in in combination with 'prevalence', 'distribution', we have limited SUT statistics between 2013 -2017, and dealing with comprehensive tire exposures and traumatic service.

Resuls: Recent community studies show that trauma exposure is higher in low-income countries compared to high-income countries. The highest rates found in post-conflict settings. Trauma, PTSD risk factors are distributed differently in low-income countries than in high-income countries, which contribute most to this risk. Care is either very low or not at all.

Conclusions:Trauma exposure is also associated with some chronic physical conditions. These findings indicate a high burden of trauma exposure in low-income countries and in post-conflict settings, where access to trained health professionals is usually low.

Keywords:Epidemiology, Post Traumatic Stress Disorder.

$O P-127$

Nursing Care in Patients with Ocular Trauma

${\text { Violeta } \text { ZANAJ }^{1 *} \text {, Arben TUSHA }}^{2}$

Authors Information:

Lecturer at Department of Medical Technical

Sciences, Faculty of Professional Studies

"Aleksander Moisiu" University Durres, ALBANIA.

\begin{abstract}
Introduction: Eye trauma is one of the most popular causes of vision loss in the developed world. Open or penetrating eye trauma can lead to vision loss through cataract or glaucoma. The etiologies of eye injury vary in urban areas compared to other settings, and vary from place to place, between different regions of the world and between different demographic or socioeconomic classes. It consists in recognizing the features of this pathology, recognizing and preventing its etiological factors before it occurs and appearing and aggravating it.

Treat every person, patient affected by ocular trauma based on anamnesis, diagnosis, medical therapeutic treatment. Nursing staff assist with physical assessment, possible nursing diagnoses, planning, nursing interventions to reduce the time for recovery. therapies and advice on avoiding its etiological factors.
\end{abstract}

MATERIAL \& METHODS: The clinical signs of ocular trauma are widely recognized; Prescribing and correctly applying the five stages of nursing to the patient: To accurately describe the imaging diagnostic tool for ocular trauma; Data on persons, patients, when they are most affected by ocular trauma, and how they experience it.

Conclusions: The nurse manages to accomplish the 5 stages of nursing in a patient with ocular trauma. He / she manages to identify the characteristic signs of ocular 
trauma and formulate his or her nursing diagnoses. Perform and applies treatment safely as prescribed by the physician. Advises the sick patient on what to do and what not to do.

Keywords;Eye trauma, ocular trauma, nursing diagnoses.

$O P-128$

\section{Managing Patients in Trauma Emergency Department}

Natasha
(HYSKO)

Authors Information:

${ }^{1}$ Nurse Service University Hospital of Trauma, Tirana, ALBANIA.

\begin{abstract}
Background:Emergency Admission Service has been nationwide since 1993 with three Ministries, Education, Health, Defense. Where he also received the appointment S.U.Q.U. In 2013 with DCM I switch to SUT. Me 2015 with decisions onThe Government is also integrated into the Military Sp. On the urgency of admission there are an average of 50-70 cases per 24 hours, of these 4-5 cases with politrauma every day. Some come directly and many more from district hospitals. Emergency patient management often faces times with different problems. This reference aims to highlight various complaints, suggestions and possible solutions to the urgency of SUT has been around for almost 23 years and is the only one in the National range.
\end{abstract}

Material and Methods:A sectional study was performed on over 300 patients in trauma emergency for a period of 2 months, from1.11.2016.to 31.12.2017

Results;Men are mostly the main victims, and road accident are the leading causes. Injuries include more than 2 specialties, coordination between different specialties in patient management in a timely manner.

Conclusion:A well-trained team specializing in trauma management with a standard procedure, standard protocols well, are the keys to an effective management of poly trauma patients.

Keywords: Polytrauma, selection, Emergency admission, triage.
$O P-129$

\section{Nursing Ethics in Trauma Patients}

Deonada ÇOKU¹, Natasha MERKO', Hasije DURAKU$^{1}$, Odeta MISHO${ }^{1}$, Etleva RAMALIU ${ }^{1}$

Authors Information:

Nurse Service, University Hospital of Trauma, Tirana, ALBANIA.

\begin{abstract}
INTRODUCTION: The word ethics is derived from the ancient Greek ethos - habits, customs and ethics - $\mathrm{i}$ moral: Ethics is the science of morality (no, moris- the Latin word for habit, adet). The purpose is to give a clear description of the concepts and terms of ethics in the field of nursing. Ethics is a very broad field, and has been a key pillar of medicine in all periods of history around the world, whose recognition and study it is much needed while practicing the profession for all health staff this paper has emphasized the importance of ethics in general, which has an impact on staff performance health, the aim is to evaluate the knowledge, attitudes and experiences of nurses in relation to with healthcare ethics and legal rules in the field of nursing: delivery of ways to formulate answers to questions and guide actions. Offers one a framework for resolving issues, problems and conflicts. An essential issue of this paper is the ethical nursing attitude towards patients at SUT
\end{abstract}

Materials and Methods: The study is of a qualitative nature, included in the study total 24 nurses of Reception, Consultation, Emergency at SUT, and is done their questionnaire survey consisted of 10 questions.

Results: Nurses with regard to knowledge about ethics have claimed that according to the belief

they possess high knowledge of ethical responsibilities in the field of nursing, and that they have gained this knowledge from work experience, university studies and attendance at training, courses, conferencing, ethics plays an important role in the professional work of health care personnel, but must always respect and work ethically. 
In this paper, we place special emphasis on knowledge, experience and

ethical attitudes of Nursing care professionals. The importance and the irreplaceable role of health care professionals in care for patients, a skilled, motivated, aware staff is a condition for development and

the proper functioning of their internships in health care institutionsthe necessary patient services while always adhering to ethical norms.

Keywords:communication, conflict, solutions, nursing ethics, the role of nurses.

$O P-130$

Management of Hypoxemia in Severe Asthmatic Status in ICU. A Case Report.

Alma CANI ${ }^{1}$, Fahri KOKIC I ${ }^{1}$, Eliana SHIMA ${ }^{1}$, Loreta AGOLLI ${ }^{2}$

Authors Information:

${ }^{1 A n e s t h e s i o l o g i s t ~ a n d ~ I n t e n s i v e ~ C a r e ~ S e r v i c e, ~}$ University Hospital "Shefqet Ndroqi", Tirana, ALBANIA

2Pneumologi Service, University Hospital

"Shefqet Ndroqi", Tirana, ALBANIA

\begin{abstract}
Introduction:Acute asthma is an episode of progressive increase in shortness of breath, cough, wheezing, or chest tightness, or some combination of these symptoms. If not treated immediately there will occur increase in flow resistance causing increased work of breathing, gas exchange inefficiency, respiratory muscle exhaustion and finally hypercapnic and hypoxemic respiratory failure. Acute severe asthma carries a high morbidity and mortality.
\end{abstract}

Case Report: A 24-year-old female presented to the hospital emergency room with breathing difficulty. The patient was a known case of bronchial asthma since years. The patient presents one-day history of shortness of breath, cough, chest pain.

At the beginning presented to the hospital emergency room of UHC "Mother Teresa". After CT thorax, results Pneumediastinitis and transferred to the hospital UH "Shefqet Ndroqi". The patient has a history of 5 years with regularly spotted bronchial asthma...
Conclusion: Acute severe asthma carries a high morbidity and mortality. It may be classified as mild/moderate/severe or life threatening. Peak Expiratory Flow rate is a good indicator of severity of the attack. $\mathrm{B}_{2}$ agonists by nebulization are the first line of therapy for acute severe asthma. Nebulization with anticholinergics is an adjunct and has no role as the sole medication in an acute asthmatic attack. Steroids are to be given in all patients, but antibiotics to be prescribed only where indicated.

Keywords: Asthma, mechanical ventilation, pneumomediastinum, acute respiratory failure.

$O P-131$

Anesthesia and Perioperative Management in Liver Resection, our Experience.

Majlinda NAÇO 1, Haxhire GANI 1, Etmont ÇELIKU², Nertila KODRA ${ }^{1}$, Anna MANDI ${ }^{1}$, Henri KOLANI ${ }^{2}$, Eden NAÇO ${ }^{3}$.

\section{Authors Information:}

${ }^{1}$ Departament of Anesthesia and Intensive Care in General Surgery, University Hospital Center "Mother Theresa", Tirana, ALBANIA.

"Department of General Surgery,University Hospital Center "Mother Theresa", Tirana, ALBANIA.

${ }^{3}$ Medical student of Catholic University "Our lady of good counsel", Tirana, ALBANIA.

\begin{abstract}
Introduction:The patients with colorectal adenocarcinoma very often developed hepatic metastases. Resection of them still remains the best treatment of disease. Hepatectomy carried about $20 \%$ mortality rate. The most important intraoperative factor is major hemorrhage and hypotension and postoperatively the main reasons are hemorrhage, coagulopathy, renal failure, pulmonary, and cardiac disturbance till biliary fistula and liver failure. Anesthesia and perioperative management have resulted in diminished value of mortality and morbidity at the same time.
\end{abstract}

Materials and Methods:In that retrospective study we enrolled 6 patients ASAII- III, from January to July 2019. All patients treated for liver malignancy diagnosis after colorectal carcinoma in elective surgery and received general anesthesia with fentanyl $1-2 \mu \mathrm{g} / \mathrm{kg} / \mathrm{h}$, 
profolol $4.0 \mu \mathrm{g} / \mathrm{kg} / \mathrm{min}$ via target-controlled infusion and vecuronium $0.001 \mathrm{mg} / \mathrm{kg} / \mathrm{min}$ and sevofluran $2 \%$. All patients are monitored with continuous central venous pressure (CVP). We recorded mean artery pressure (MAP), heart rate (HR), CVP, amount of transfusion, incidence of complications and discharge from intensive care and hospital.

Results:Female/male report was $66,6 \%$ vs $33.4 \%$. The age of patients were $53-72$ years with a mean \pm SD age of $65.1 \pm 9.5$ years. CVP was 0-5. Amount of blood was 2.5 unite during innervation. Average of days in intensive care was 3.83. Renal compromised was $3 \%$. Ascites and edema were $50 \%$. All patients were discharged home after 14.66 days.

Conclusion:Optimizing hemodynamics and fluid administration and reduced the administration of blood therapy using low CVP (0-5).

Keywords:blood transfusion, CVP, liver surgery, perioperative management.

$O P-132$

\section{Management of Acute Cardiac Tamponade Following Cardiac Procedures.}

Alfred Ibrahimi ${ }^{1}$, Saimir Kuci¹, Ervin Bejko, StavriLlazo ${ }^{1}$, Esmeralda Bulku${ }^{1}$, Jonela

Burimi $^{1}$

Authors Information:

${ }^{1}$ Anesthesia and intensive care service, in cardio-vascular clinic "Mother Theresa" Hospital, Tirana, ALBANIA

\footnotetext{
Abstract

Cardiac tamponade is defined as a hemodynamically significant cardiac compression caused by pericardial fluid. The number, specific type, and complexity of percutaneous cardiac procedures continue to evolve, most common causes of acute cardiac tamponade are: rupture of aortic dissection, coronary laceration during PCI procedure and massive hemopericardium after pericardiocentesis. Tamponade is an absolute indication for urgent drainage, either by pericardiocentesis or surgical pericardiotomy.
}

For nine consecutive months 10 cases of acute cardiac tamponade due to hemopericardium was recovered in ICU of cardiac surgery. All the patient were in cardiogenic shock and immediately 9 of them was performed median sternotomy and one pericardiocentesis with insertion of central venous catheter. In 9 nine cases perforation of coronary artery was founded. One patient died due to heart failure after CABG.

Conclusion: In the era of increasing numbers of cardiac invasive procedures and the numbers of complication such an acute cardiac tamponade also increases. Aggressive treatment in ICU and immediate drainage of pericardium are lifesaving procedure for the patients.

Keywords:Cardiac tamponade, intensive care unit, peri cardiocentesis, surgical

pericardiotomy.

$O P-133$

Pneumoperitoneum after open Heart Surgery.

Alfred IBRAHIMI 1, Saimir KUCI ${ }^{1}$, Ervin BEJKO 1, Stavri LLAZO 1, Selman DUMANI $^{1}$

Authors Information:

${ }^{1}$ Anesthesia and intensive care service, in cardio-vascular clinic "Mother Theresa" Hospital, Tirana, ALBANIA

\begin{abstract}
Introduction:Because of the close anatomic relationship of the pericardium, diaphragm, and peritoneal cavity, a benign pneumoperitoneum may occur following median sternotomy incision and cardiac surgery. The pneumoperitoneum results from inadvertent opening of the peritoneal cavity during the initial surgical incision or during the subsequent cardiac surgery. It is generally of no significance to the patient, although it must be distinguished from pneumoperitoneum occuring secondary to intraabdominal pathology or to retrograde dissection of extra alveolar air in patients on ventilatory support. One illustrative case is
\end{abstract}


presented. Patient after reoperation for mitral valave repalacement in decompesation situation massive ascites in abdominal cavity was seen. After that massive bilateral pleural efusion was removed for 4 consecutive days. Pneumoperitoneum, without, peritoneal signs and pneumomediastin was diagnosed. Air passes from open sternal wound to mediastinum and peritoneal cavity through normal anatomic ways. After closure of sternal wound, air was absorbed in thoracic and abdominal cavity

Keywords:pneumoperitoneum, sternotomy, cardiac surgery, pneumomediastin

$O P-134$

Hydroxocobalamina is a Rescue Treatment for Refractory Methylene Blue Resistant Vasoplegic Syndrome During Cardiac Surgery A Case Report.

Marjeta BILAJ(Miraka) ${ }^{1}$; B BARRIC ${ }^{2}$

Authors Information:

${ }^{I}$ Dept of Anesthesia and Critical Care at American Hospital, Tirana, ALBANIA.

${ }^{2}$ Dept of Anesthesia, Cardiothoracic division at UNC, Chapel-Hill, NC USA

\footnotetext{
Abstract

Introduction: Vasoplegic syndrome (VS) is a complication during cardiopulmonary bypass (CPB) (25\%) and has a significant morbidity and mortality especially in the $5 \%$ of these patients non-responsive to conventional vasoconstrictor therapy. In such refractory vasoplegia manifests with hypotension, low systemic vascular resistance and normal or increased filling pressures. Conventionally methylene blue has been the drug of choice and effective as rescue therapy. It may not be effective in all patients. Recently hidroxocobalamina is effective and an alternative method of managing refractory vasoplegia even in methylene blue resistant VS. We report a case of VS during CPB and a successful managing Treatment.

Case Report: We describe a young male patient, in chronic hemodialysis with infective endocarditis undergoing tricuspid valve repair and aortic valve replacement surgery. Standard ASA monitoring and general anesthesia was induced with propofol, fentanyl, midazolam and cistracurium to
}

facilitate intubation. When being weaned on cardiopulmonary bypass, the patient experienced VS, hypotension during an immediately following CPB. The patients' blood pressure did not increase after the administration of a high dose of vasopressor use, epinephrine vasopressin and norepinephrine. Therefore, he was initiated on a dose of methylene blue $2 \mathrm{mg} / \mathrm{kg}$ but his blood pressure didn't reach the normal range. In conclusion the decision was made to administer intravenously hidroxocobalamina $5 \mathrm{~g}$ through the CPB circuit and within 20' of administration the patient was separated successfully from CPB in a stable condition with a low dose of vasopressor support. On arrival to the ICU the patient was token off completely from vasopressor (norepinephrine) and did not require additional vasopressor boluses. On postoperative day 3 the patient was transferred to the cardiac step-down floor in a good hemodynamic condition.

Conclusion: This is the case of VS on CPB unresponsive to vasopressor support and methylene blue whereas responsive to hidroxocobalamina which potentially indicates that the effect of hidroxocobalamina is significant or that the two drugs have synergic effects with one another in one or multiple mechanisms.

Keywords: Hidroxocobalamina, vasoplegic syndrome, CPB, vasopressor.

$O P-135$

Anesthesia Challenges in Sleeve Resection in Thoracic Surgery

Rinard KORTOCI ${ }^{1^{*}}$, Safet BEQIRI ${ }^{2}$, Blerim ARAPI $^{1}$, Roland KAZA ${ }^{1}$, A BAKO ${ }^{1}$, Marjeta BILAJ $^{1}$, A CANE ${ }^{1}$, A JAUPI ${ }^{1}$.

Authors Information:

${ }^{1}$ Anesthesia Department American Hospital, Tirana, ALBANIA.

${ }^{2}$ Thoracic Surgery Department, Kerckhoff Clinic Bad Nauheim GERMANY.

\begin{abstract}
Introduction: Sleeve resection and especially tracheal reconstruction requireexperienced team and good skills. Most of the cases are
\end{abstract}


non-small cell lung tumour and sleeve lobectomy is performed when is possible tosave pulmonary tissue,

Material and Methods: During 2018-2019 have been done 5 patients, and all are males'non-smallcell pulmonary carcinoma. Mean age $63+-6$ year.

Four were sleeve lobectomy (one was post stenotic abscess and post chemotherapy) and one of them was right pulmonectomy with tracheal reconstruction. Postern-lateral thoracotomy was used for all patients and "compartment lymphadenectomy". We use double lumen tubes (one lung ventilation), total intravenous anesthesia (TIVA) propofol + remifentanyl

All patients are extubated in OR. Monitoring includes: Respiratory rate, capnometry, Invasive blood pressure, ECG, SpO2, temperature, urine output.

Results: All are discharged at $8^{\text {th }}$ postoperative day (except the patient with pulmonary abscess 15days). We have documented all the anastomosis after four weeks under the flexible bronchoscopy.

Conclusions: Most challenged moments are: correct position of the tube under flexible bronchoscopy, apnoeic hyperoxygenation, lung protection ventilation, ventilation from the surgical field and emergency extubating.

Keywords:Sleeve resection, carinal reconstruction, TIVA, apneic hyperoxygenation.

$O P-136$

\section{Preservation of Fingers Length after Traumatic Amputations}

Sokol ISARAJ ${ }^{1 *}$, Nardi KOLA ${ }^{1}$, Albana ALEKSI $^{1}$, Olivia SHABANI ${ }^{1}$, Ina KOLA ${ }^{1}$

Authors Information:

${ }^{1}$ Service of Burns and Plastic Surgery UHC "Mother Teresa" Tirana, ALBANI

Abstract

Introduction: Traumatic amputations rarely occur in scenarios other than combat situations; however, when they do occur, they are emotionally and physically debilitating.
Success in replantation depends on appropriate and time-sensitive care of the patient during the initial presentation to a health care facility, usually the emergency department (ED). It is the responsibility of the emergency care provider to know how to manage amputations and initiate proper care that will lead to a satisfactory outcome The adage "life over limb" accurately describes the priorities in the prehospital care of traumatic amputations. ${ }^{7,8}$ The prehospital care provider is tasked with caring for not only the injured patient but also the amputated part. Stabilization of life-threatening injuries, control of hemorrhage, and appropriate storage of the patient's arrival at the ED is usually chaotic, as patients with an amputation can be distraught. They may be agitated and combative, flailing a partial amputation around and, thus, making their injury worse. Surgical Techniques:Depending on the type and location of the amputation and the length of time since it occurred, the patient may be a candidate for replantation. The amputated part suffers similar physiologic derangements as the patient in shock. The goal of replantation is to provide a functional part with reasonable sensation and mobility.

Conclusion:Traumatic amputations are devastating and debilitating injuries. The results of replantation are rarely perfect, but with a time-efficient and pragmatic approach to management, satisfactory cosmetic and functional outcomes can be achieved.

Keyword: injury, trauma, amputation,replantation.

OP - 137

The First Dorsal Metacarpal Artery in the Treatment of the first Web Space Burn Wounds of the Hand

Gentian ZIKAJ ${ }^{1 *}$, Gjergji BELBA ${ }^{1}$, Sokol ISARAJ $^{1}$, Gezim XHEPA ${ }^{1}$, Nardi KOLA ${ }^{1}$.

Authors Information:

${ }^{1}$ Burns-Plastic Service, University Hospital Center "Mother Teresa" Tirana, ALBANIA.

\section{Abstract}

Introduction:Electrical injury often causes deep burns involving underlying soft tissue and bone. Other associated complications 
include vascular and neurologic injuries, fracture, subluxation of joints. Rapid resurfacing of the wound inessential to enable early mobilization and optimum recovery of hand function. Description: A 34-yearoldright-hand-dominant man sustained an electrical injury to his left hand. He presentedwith deep burns to the firstweb space and the dorsalaspect of the index finger. Theexcision of the escharresulted in exposure of baretendons. FDMA flap wasdone. Around three monthslater, the patient had normalhand function.

Discussion: If the resultingtissue is limited to superficialsoft tissue loss, a skin graftmay be sufficient forcoverage. However, if there isexposure of the deeperstructures including loss ofperitenon, the resulting defectshould be closed with local orregional flaps or free tissue

transfer. Island FDMAF is areliable option with many advantages. It does not involve sacrifice of any majorvessel. It provides acceptablefunctional and cosmeticoutcomes regarding skinmatch, elasticity, durabilityand sensitivity. So, it may be considered \& novel use.

Conclusion:The FDMA flap is a versatile flap for coveringfirst web space defects ondorsal or volar aspects. Thisflap can perform with a minimal donor site morbidity which provides sensation, length, stability, mobility as well as aesthetics.

Keywords:Hand burn, epidemiology, treatment, outcome.

$O P-138$

The Reconstruction of the Crural Soft Tissues with Reverse Sural Flapafter the Pathologic Tibial Fracture in the post Combustional Abandoned Wound - A Case Report.

Gëzim XHEPA, Gentian ZIKAJ.

Author Information:

Department of Plastic and Burn Surgery, at University Hospital Center "Mother

Theresa", Tirana, ALBANIA

\section{Abstract}

The patient H. A., 57 years old, from Durrës, is hospitalized in the "Clinic of Plastic and Burn Surgery" on January of 2018, with diagnosis: "Pathologic tibial fracture, from the bone exposition in an abandoned burn wound region". The patient is transferred to us from the Orthopedic Clinic of the Durrës Regional Hospital, to cover the exposed bone with soft tissue for the healing of the tibial bone.

The patient had suffered a pathologic fracture 3 weeks ago, after a ten months period of the exposition of the distal $1 / 3$ of tibia in an abandoned burn wound, for which was treated with external fixation device. In the conditions of a aerobic devitalization of the bone segment, our college asked the transfer of the patient in our clinic, to cover fractured bone with vital well vascularized soft tissues. Although, in our experience, the random adapt and safety reconstructive technique, in this case would be the reverse sural flap, the undertaking of the therapeutic decision was very difficult, because of the split skin graft application in the whole crural region with the accompanied pathologic fibrotic tissue in the donor and recipient flap region.

The flap was outlined in the form of the traumatic defect and slightly larger in size. The flap pedicle was calculated to cover the defect after the rotation. The axis of the flap rotation was calculated about $150^{\circ}$ to facilitate the venous drainage.

The flap was elevated respecting the standard technique in the subfascial plane, under the gastrocnemius fascia propria, with a $4 \mathrm{~cm}$ wide pedicle in a length till $7-8 \mathrm{~cm}$ over the lateral malleolus.

The patient was transferred after 3 weeks for the further treatment in the Orthopedic Clinic of Durrës Hospital.

As conclusion, we can say, that the reverse sural flap is one of the most safety flap in the correction of the defect of the distal third of the leg, and in pathologic local condition of the subcutaneous fibrotic alteration, after the burn trauma.

$$
O P-139
$$

Management of Auricular Traumas in The Emergency Setting

Rezarta KAPAJ*$^{1}$, Albiona
Mariola NDRECKA

Authors Information: 
${ }^{1}$ Plastic Surgery Department, American Hospital, Tirana ALBANIA

\begin{abstract}
Introduction: The external auricle has a high potential for injury due to its exposed and unprotected position alongside the head. A retrospective study by Bardsley and Mercer looking at hospital records in auricular injury cases revealed that human bites constitute the most common cause of injury (42\%). This was followed by falls (20\%), automobile accidents (16\%), and dog bites (14\%). The most common injury observed was incomplete amputation of the ear, usually helical rim tissue loss. Untreated open auricular injuries invariably result in infection, ensuing deformities, and further tissue loss.

Auricle avulsion or amputations may present with the complete involved segment or a portion thereof available for reconstruction. Isolated traumatic ear amputation is an uncommon event and frequently occurs in conjunction with major systemic or head and neck trauma.
\end{abstract}

Material and Methods: We present three cases we have treated in our setting presenting with partial or near total ear amputation. The etiology of the laceration was: cut with a glass, cut with a knife and sports injury. All three patients were male. The average age of the patients was 20 years old. Two of them arrived at the hospital within hours of the accident while the third arrived 40 hours after partial total ear amputation and restore of the amputated segment. The management of the cases and final results are presented.

Results: Satisfactory to excellent results were achieved in the two cases presented immediately after the injury. The final result remains to be seen in the third case.

Conclusions: Ear lacerations present a challenge for the reconstructive surgeon because of the intricate anatomy and the visibility of the ear. Final results depend on the degree of laceration, timing and type of the primary care and the general status of the patients. as such education of the primary care personnel is mandatory.

Keyword:Auricle injury, restore, Bardsley and Mercer, plastic.
$O P-140$

\section{Pia Flap in Hand Trauma Defects Reconstruction}

Nardi KOLA ${ }^{* 1}$, Sokol ISARAJ ${ }^{1}$, Albana ALEKSI $^{1}$

Authors Information:

${ }^{1}$ Service of Burns and Plastic Surgery UHC

"Mother Teresa" Tirana, ALBANIA

\begin{abstract}
Introduction:Soft tissue reconstruction of the dorsum of the hand is always a complex problem to deal with because of the involvement of muscle, tendon and bone. There are different flaps at our disposal that can be used to cover these soft tissue defects. This flap includes local perforator-based flaps, reverse flow forearm flaps, distant flaps like the groin and abdominal flaps and free flaps. Each of these flaps has their advantages and disadvantages.

The reverse posterior interosseous artery (PIA) flap is based on reverse flow through the PIA via anastomosis with the anterior interosseous artery and the dorsal carpal arches near the wrist. The distal reach of the PIA flap is also limited to the MCPJ of the fingers and the IPJ of the thumb. This work presents our experience with the use of reverse posterior interosseous artery flap for reconstruction of post trauma soft-tissue defects the dorsum of the hand.
\end{abstract}

Material and Methods:5 distally based reverse flow posterior interosseous flap flaps were performed for hand reconstruction between 2010 and 2015. Each patient received a full surgical explanation and a written consent was obtained. All patients were males. Their ages ranged from 35 to 57 years old. The reconstructed areas included the dorsum of hand 4 cases and the first web space in one case. Two of the cases were post electrical burn sequelae, two other road accidents and one of them gun related injury. We measured in all of them the pedicle length, the artery diameter and the defect length. We decided to establish a one year period of follow-up for all the patients to see the flaps conditions after time passing.

Results:We had complete survival of the flap in four of the cases and only one partial survival. We had to deal with flap bulking in 
one of the cases after six months. The patients satisfaction varied from 7 to 9 in a scale from 5 to 10 .

Keywords: Reconstruction, soft tissue, hand.

$O P-141$

Indications of Lasers Treatment for Lymphatic Malformations

Vladimir FILAJ *, Gjergji BELBA

Authors Information:

Service of Burns and Plastic Surgery" Mother Theresa" of Tirana. ALBANIA

\section{Abstract.}

The main therapeutic

optionsfor treating a lymphatic

malformation are percutaneous drainage,

surgery,sclerotherapy, laser therapy,

radiofrequency ablation, or medical therapy.

Cutaneous dermatologic lesions of lymphatic

malformation, in type of superficial,

translucent, localized or diffuse vesicles, are

accessible to laser treatment. The useful

lasers are essentially laser $\mathrm{CO}_{2}$, and rarely

pulsed dye laser and the Nd: YAG.

Treatment by laser allows to obtain the

ablation of vesicles by limiting

aftereffects(scare) and so to dry up the

transcutaneous lymphatic liquid.

However, laser has no action on the deep

constituent of the lymphatic malformation

and it's why medium and long-term

recurrences are inevitable.

Finally, the indication of the laser must be retained only as a supplement to the surgery and has to be done by experimented persons.

Keyword: lymphatic malformation, laser treatment.

$O P-142$

\section{Burns in Infants.Modern Trends inManagement of Fluids During the Resuscitation.}

Albana ALEKSI ${ }^{1 *}$, Adriatik LAJÇI ${ }^{2}$

Authors Information:

${ }^{1}$ The Service of Burn and Plastic Surgery, UHC Tirana, ALBANIA

${ }^{2}$ Resident of Surgery, University Clinical Center of Pristina, KOSOVO

\begin{abstract}
Introduction: Burns are one of the common injuries of childhood. Because of the potential severity of their residual deformities, burn injuries in infants justify an early management in specialized centers when they cover more than $5 \%$ of body surface and in every case when hands, face, or external genitalia are concerned. The risk of death from a major burn is associated with increased burn size, decreased age, the presence of a full-thickness burn, the presence of inhalation injury.
\end{abstract}

Material and Methods: The current article is a case report and will review the management of ongoing effective treatment of the first phase: the resuscitation. This article is e part of a large study about the challenge of treatment in the phase of burned shock with colloids and plasma for a good judgment of a good resuscitation and in final of a good outcome.

Conclusions:Management of this age of pediatric burn patients remains a difficult challenge for clinicians from clinical, social and ethical perspectives. General treatment includes fluid and electrolyte therapy, temperature control, appropriate nutrition and pain suppression. Pain suppression is a major part of the treatment and morphine must be largely used. Chemical or electrical burn, radiation, associated $\mathrm{CO}$ intoxication or multiple trauma, as well as burn injury in infants, raise specific problems.

Keywords:Burns, burned shock, colloids, resuscitation.

$O P-143$

\section{Inguinodinia in Young People}

Kastriot HAXHIREXHA ${ }^{1 *}$, Sadi BEXHETI 1, Lutfi ZYLBEHARI 1, XheladinELEZI 1, Ferizat DIKA - HAXHIREXHA ${ }^{1}$,

Authors Information:

${ }^{1}$ Department of Surgery, Clinical Hospital Tetovo, RN of MACEDONIA.

\section{Abstract}

Introduction: Groin pain syndrome usually describe the concern which is frequently encountered among young people. This concern is characterized by onset of pain across the inguinal canal region and 
sometimes is aggravated by physical activity. Purpose is to represent our experience with the treatment of long-term inguinal pain in young people.

Material and Methods:This is a retrospective study in which we have included eighty-nine young people aged between 12 and 18 years. All of them complained of long-term pain along the inguinal region.

Results:All the patients included in this study were treated because of chronic embarrassing pain. In seventy of them the treatment was conservative with administration of minor analgesics of NSAID groups. In the seventeen others we performed hernioplasty sometimes followed by ilio- inguinal nerve neurolysis and resection of the genital branch of genitofemoral nerve, whereas in two patients because of the size of the hernia the tension free hernioplasty using a prolene mesh was the method of choice. Patients were discharged from day one to five after the operation and no any serious postoperative complications were observed. All the patients were discharged from the hospital two to four days after operation and no one complained of any other pain or distress. The patients were observed long-term after surgery

Conclusion:inguinodinia in young people may be a very distressful problem. In some patients the pain can be resolved conservatively with NSAID group analgesics, whereas in most of them hernioplasty sometimes combined with resection of the genital branch of genitofemoral and ilioinguinal nerve (neurolysis) is the best treatment procedure.

Keywords:young people, inguinodinia, hernioplasty, neurolysis

$O P-144$

\section{Recurrent Achalasia}

Ilir SHANI, Aldi SHANI ${ }^{2}$, Frenki VILA ${ }^{2}$, Eljona XHELILI ${ }^{3}$.

Author Information:

${ }^{I}$ General Surgeon at Durrës Regional Hospital, ALBANIA

${ }^{2}$ Resident in General Surgery at University Hospital Center "Mother Theresa", Tirana, ALBANIA
${ }^{3}$ General Surgeon at Kukës Regional Hospital, ALBANIA

\section{Abstract}

Achalasia is characterized by impaired LES relaxation and by the lack of peristalsis in the distal esophagus it is accompanied by the loss of myenteric ganglion of the distal esophagus. it is not yet known why myenteric plexuses degenerate. is supposed to be an autoimmune process driven by a viral infection on a genetically vulnerable subject.

Heller myotomy achieves a significant improvement in patient signs but 5-year barium surveillance shows relapse is not uncommon and goes up to $90 \%$ for the third type. $69 \%$ of patients after surgery have at least one symptom after myotomy. The study reviewed the literature for 2013 to 2019 and assessed the role of Redo Heller. Botox Poem and esophagectomy. There are few studies of reintervention after Heller myotomy in patients with achalasia. Therapeutic Dilemma: What to Do After a Heller Myotomy? Balloon? Botox? , Poem? Esophagectomy? 69\% of patients after surgery have at least one symptom after myotomy. Reintervention in some studies over 5 years goes up to $62 \%$ for the first type, $74 \%$ for the second type, and $87 \%$ for the third type. After reintervention the chances of reintervention are $50 \%$ for the next 2 years. An annual barium survey is recommended.

Keywords:Achalasia, myotomy, balloon, esophagotomy.

$O P-145$

The Surgical Treatment of Secondary

Hepatic Metastases from Colorectal Carcinoma.

Henri KOLANI ${ }^{1 *}$; Frenki VILA ${ }^{2}$; Eriol BRAHOLLI $^{2}$; Eljona XHELILI ${ }^{3}$; Bledi MASATI $^{3}$; Asfloral HAXHIU ${ }^{3}$; Etmont CELIKU ${ }^{4}$

Authors Information:

${ }^{I}$ General Surgeon, University Hospital Center

"Mother Theresa" Tirana, ALBANIA

${ }^{2}$ Resident in General Surgery, University Hospital Center "Mother Theresa", Tirana, ALBANIA

${ }^{3}$ General Surgeon in University Hospital Center

"Mother Theresa", Tirana, ALBANIA

${ }^{4}$ General Surgery in University Hospital Center

"Mother Theresa" Tirana, ALBANIA 


\section{Abstract}

Introduction:Colorectal cancer is the third most common cancer and the third leading cause of mortality among men and women in our country, and is represented by a vast number of cases diagnosed and treated in our clinic slightly second to gastric cancer. More than $50 \%$ of the patients with colorectal cancer (CRC) have or will develop metastasis, with a quarter having distant metastatic disease at the time of diagnosis, most frequently in the liver.

Liver metastasis is the leading cause of cancer-related morbidity and mortality in colorectal cancer. The only potentially curative treatment for liver metastasis is liver resection, but only $15 \%$ to $20 \%$ of the patients are suitable for surgical resection. Regardless the early diagnosis and the treatment strategy the survival rarely exceeds the 3-year period.

Treatment: Surgical resection remains one of the major curative treatment options available to patients with colorectal liver metastases. Surgery and chemotherapy form the backbone of the treatment in patients with colorectal liver metastases.This article provides an overview of the surgical management of colorectal liver metastases.

Conclusions: According to the data from retrospective and comparative studies the surgical resection remains the best choice of treatment followed by higher rates of survivability. Our experiences with cases operated with synchronous and metachronous liver metastases secondary to colorectal cancer, shows an increase in survivability by 25 to $50 \%$, and in the meantime, there is no positive data in the conservatory treated cases limited to chemiotherapy.

Keywords: Hepatic metastases; Colorectal cancer; Surgical resection.

$O P-146$

Acute Colonic Obstruction

Sadık Yıldırım Özgeçmiş

Author Information:
General Surgery, Oncologic Surgery, Oncology Center, Istanbul, TURKEY

\begin{abstract}
Acute colonic obstruction is a medical/surgical emergency because of the potential for bowel ischemia, perforation, and sepsis with peritonitis if not rapidly and appropriately treated. There are numerous causes of acute colonic obstruction, which must be differentiated from colonic pseudoobstruction, which also is considered a medical emergency. Management options include medical therapy, surgical therapy, endoscopic therapy, and interventional radiologic therapy. Self-expandable metal stents (SEMS) have gained acceptance for alleviating acute malignant colonic obstruction and in some situations for preoperative relief of acute benign colonic obstruction. In this talk I will review the approach to the patient who has acute colonic obstruction.
\end{abstract}

$O P-147$

Fasciitis necrotic, morbus Fournier is it a surgical emergency?

\author{
Astrit XHEMALI ${ }^{1 *}$, Arvit LLAZANI ${ }^{1}$, \\ Myzafer KAÇI ${ }^{1}$, A. MASKA, L. Laci, L. \\ BYLYKBABSHI
}

Authors Information:

Department of Surgery, University Hospital Center "Mother Theresa" Albania

\begin{abstract}
INTRODUCTION:The authors report their experience in diagnosis and treatment of one case of Fournier's gangrene; recent international literature review.

Complete clinical report. Diagnostic, clinical and prognostic indication, evaluation of effectiveness of surgical treatment (debridement and necrectomy) and followup; comparison between indications and multidisciplinary approach proposed by international literature.

Surgical treatment previous multimodal approach, according to international guidelines. Fournier's gangrene is an uncommon
\end{abstract}


and aggressive synergistic fasciitis of the perineum and genital organs, which may bring the patient to death; it is a true surgical emergency.The disease can no longer be considered to be idiopathic; in most cases a urologic, colorectal or cutaneous source canbe identified. Despite antibiotics and aggressive debridement, the mortality rate remains high, particularly in the elderly, in patients with renal failure, and in patients with extensive disease.

The presentation is highly variable, necessitating a high index of suspicion. High risk patients include diabetics, alcoholics and debilitated and immunosuppressed individuals. Broad spectrum antibiotics and aggressive debridement remain the hallmarks of treatment. The surgical operation has to be performed in emergency to avoid a rapid spread of tissue necrosis and a possible development towards septic shock.

Keywords:Fournier's gangrene, debridement, anaerobic, surgical emergency.

$O P-148$

Unusual Abdominal Wall Abscess as a Consequence of Cecal Adenocarcinoma. A case reports.

Kastriot HAXHIREXHA ${ }^{1 *}$, Sadi BEXHETI 1, Lutfi ZYLBEHARI ${ }^{1}$, Ferizat DIKA HAXHIREXHA ${ }^{1}$, Aulona HAXHIREXHA ${ }^{1}$

Authors Information:

${ }^{1}$ Department of Surgery, Clinical Hospital Tetovo, RN of MACEDONIA.

\footnotetext{
Abstract

Introduction: In old people the right lower abdominal pain must be suspected for right colonic and cecal tumor especially if the anamnestic data point to other complications such as anemia, weight loss and duration of illness for several months. In very rare cases this form of cancer can be complicated by abdominal parietal abscess and a presence of enterocutaneous fistula.

Case Presentation:65 years old women presented in our department with severe right quadrant pain. On examinationa large tender warm mass in the RLQ regions sized about 15 x $15 \mathrm{~cm}$ draining purulent discharge was detected. Laboratory analysis revealed the WBC 14600 Hemoglobin level $5.5 \mathrm{~g} / \mathrm{dl}$ and
}

high level of tumor markers CEA $16.3 \mathrm{ng} / \mathrm{ml}$ and Ca 19/9 $77 \mathrm{U} / \mathrm{ml}$. Abdominal computed tomography showed the presence of a large mass in the cecum which adhered to a portion of the small intestine and extending to the abdominal wall. Laparotomy through the inferior midline incision was undertaken and a large malignant cecal mass adherent to the terminal ileum was found. The tumor also affected the abdominal wall in the RLQ region. A right hemicolectomy and resection of terminal ileum was performed including a small portion of the abdominal wall infiltrated by tumor mass. Ileostomy with abdominal wall reconstruction was successfully performed and the patient had a satisfactory recovery. After the recovery the patient continued treatment at the oncology clinic

Conclusion:locally advanced colon cancer presents a real challenge for the surgeon, whereas a multimodal approach is a necessary condition to improve the patient's condition and survival.

$O P-149$

Serious Postoperative Complication after Anterior Intrapelvic Approach on the Treatment of Pelvic and Acetabular Fractures

Ilir HASANI ${ }^{1}$, Rezeart DALIPI ${ }^{1}$, Igor KAFTANDZIEV $^{1}$, Neda TRAJKOVSKA ${ }^{1}$, Simon TRPESKI ${ }^{1}$, Aleksandar SAVESKI ${ }^{1}$, Oliver ARSOVSKI ${ }^{1}$, Marko SPASOV ${ }^{1}$, Anila RACAJ $^{1}$, Jordan SAVESKI ${ }^{1}$.

Authors Information:

${ }^{1}$ University Clinic for TOARILUC - Skopje, Republic of North MACEDONIA

\begin{abstract}
Introduction:Anterior Intrapelvic Approach has been promoted as new and alternative approach on treating the fractures of the acetabulum. The intrapelvic approach was originally described by Hirvensalo in the early 90ies and a further comparable description was published shortly thereafter by Cole. Since then, various modifications have been described. Whereas the ilioinguinal approach was used until then to treat
\end{abstract}


acetabular fractures with relevant anterior column involvement from an extra pelvic view, the intra pelvic approach was developed to address the often-accompanied central hip dislocation in these fracture types with relevant fractures of the quadrilateral surface. Since we have started to use this approach, we have never used nomore the classical Letournells approach on the treatment of acetabular fracture, eventually the anterior pelvic ring fractures. With this approach a completely different view to the antero-medial acetabular pathology was possible. This approach has its specifics, on the fixation technique, instruments, implants, but also the intraoperative riscs and complications.

Materials and Methods: Consecutive group of 27 patients were treated by using the AIP approach in the period between December 2017 and May 2019.The surgeries of patients were performed at the University Clinic for Traumatology - Skopje. Fracture reduction and treatment outcomes were assessed by clinical and radiological examination. In our experience, there was no intra operative complication, only 2 patients had postoperative complication.

Results: We present two serious postoperative complications in the group of 27 patients with acetabular fractures. No complication occurred intraoperatively. One of the complications was damaged of small intestine during wound closure, on an old patient with unstable pelvic fracture. Three weeks after surgery appeared feces in the wound. After sutured the wall of small intestine, washed and sutured the wound, the patient was stable. Second postoperatively complication is an undisciplined psychiatric patient, pelvic fracture $\mathrm{C}$ type, with polyuria (cca $30 \mathrm{l} /$ per day), due to failure of the osteosynthesis material, the plate has late postoperatively damaged the urinary bladder with derivation massive amount of urine in the wound. The plate has been removed, urinary vesicle sutured, catheterization and cystostomy were performed.

Conclusion: The AIP approach seems to be safe method of treating acetabular fractures that allows good visual access and allows excellent fracture reduction. Our preliminary results revealed a much lower incidence of complications during surgery than traditional approaches, except post-operative complications.

Keywords: Anterior intrapelvic approach, acetabular fractures.

$O P-150$

Transfer of Trochanter Major Bone Flap for Treatment of Stage $I I^{B}-I^{B}$ of Femoral Head Osteonecrosis.

Ervin JONUZI ${ }^{*}$, Dmitry ILYIN ${ }^{2}$

Author Information:

${ }^{1}$ Ortopedic Surgeon, Trauma and Orthopedic Department, German Medical Policlinic, Tirana, ALBANIA.

${ }^{2}$ Ortopedic Surgeon, ECSTO Clinic, Moscow, RUSSIA.

\begin{abstract}
Introduction:Femoral head osteonecrosis or Avascular Necrosis is a condition caused by a compromise of the blood supply as a result characterized by the death of osteocytes and bone marrow.

Osteonecrosis of the femoral head (ONFH) usually affects young adults in the third and fourth decades of life. There has been an increasing trend in its diagnosis and treatment in latest years in Albania. Every year about 15,000 - 18,000 new cases are diagnosed in the United States. According to the literature, about 5\%-12\% of hip arthroplasties are performed for the treatment of this condition every year. It starts with one femoral head being affected first; bilateral involvement occurs in two years in $72 \%$ of cases.

The goal of a vascularized bone flap transfer is to replace the necrotic bone of the femoral head, restore the blood supply, and provide new bone and mechanical support for the femoral head.
\end{abstract}

Results:We are using the anterolateral approach to the hip. The incision is made 30$50 \mathrm{~mm}$. The interval between the rectus femoris and vastus lateralis is split and the transverse branch of the lateral femoral circumflex artery is identified. Carefully the pedicle is isolated and protected. The vascularized bone flap is harvested from the greater trochanter. Necrotic bone is debrided through a bone window made at the junction of the femoral neck and head. The cancellous bone from the greater trochanter is implanted, 
and the vascularized bone flap is positioned and fixed. Patients are partial weight bearing for 6 weeks. Full weight-bearing is allowed after 3 months postoperatively.

Conclusion:Patients with symptomatic ON with small lesions should be treated with head-sparing procedures. Patients with large lesions in pre-collapse hips should be treated with head-sparing procedures if the patients are young or have THA in older patients. For patients with a collapsed femoral head, THA is the recommended option.

Keyword:Femoral head, osteonecrosis, avascular necrosis.

$O P-151$

Surgical Treatment of Acromioclavicular Dislocation Grade III or IV with thigh Rope System, a retrospective study on the Clinic of Traumatology-Skopje in the period of 2015 to 2018.

Valentin VEJSELI ${ }^{1}$, M. ILIE MD ${ }^{1}$, S. KRSTEVSKI $^{1}$, Ilir HASANI ${ }^{1}$, Igor KAFTANDZIEV $^{1}$

Authors Information:

University Clinic of Traumatology, Medical Faculty, Skopje, MACEDONIA

\footnotetext{
Abstract

Introduction: Acromioclavicular (AC) dislocation is a common injury with incidence of 3 to 4 per 100000 in the general population. It is more common in younger athletic patients. In 1984, Rockwood modified Tossy et al and Allman classification to include types IV, V, and VI and we use that classification. The aim of our study is to evaluate surgical treatment of AC dislocation grade III or IV with Tight Rope system and functional results.

Materials and Methods: On the Clinic of Traumatology, Skopje in the period of 2015 to 2018 year were treated 32 patients with type III (14 patients) or IV AC dislocation (18 patients) with female to male ratio 0.45 (f:m=10:22). Inclusion criteria were applied to all patients. Three radiographic views were used: AP view, true axillary view and stress view of both sides of the AC joint and CC ligament. Surgical technique for thight rope technique was performed.
}

Results: Dash (Disabilities of the Arm, Shoulder and Hand) and VAS (Visual Analogue Scale) scores were evaluated preoperatively and postoperatively. The DASH questionnaire was used as an indicator of the impact of an impairment on the level and type of disability.

Conclusions: The technique proved to be effective in treating acute AC dislocations (Rockwood type III or IV) with a high degree of excellent and good functional results.

Keywords:Dislocation, acromioclavivular, injury, technique.

$O P-152$

Prediction of the Fractured Femur by using Quantitative Gait Analysis

Kenan BAYRAKCI ${ }^{1,}$ Onder M. DELIALIOGLU $^{1}$, Ledian FEZOLLARI ${ }^{2}$

Authors Information:

${ }^{1}$ Orthopedic Services, American Hospital,

Tirana, ALBANIA.

${ }^{2}$ Department of Orthopedic, University

Hospital of Trauma Tirana, ALBANIA.

\begin{abstract}
INTRODUCTION: Femoral shaft fractures during childhood cause femoral torsion abnormalities and significant deviations of gait characteristics. Majority of the survivors achieve an independent ambulation by the help of various compensations. However, there is no study investigating the primary gait deviations causing these compensations. Our premise was that the parameters used in compensation can be the predictors of the fractured femur.
\end{abstract}

Materials and Methods: We examined both femora of fifteen children admitted to our hospital for unilateral femoral fracture in twelve, and bilateral in three. Eight were male and seven were female. The average age was 9.56 years (5-15 years). We treated five of eighteen fractured femora by open reduction and plate-screw, eight by closed reduction and retrograde titanium elastic nail and five with spica cast. They were followed up with an average period of 22.94 months (9-48 months). At the final assessment we evaluated each patient with computerized 
tomography, radiographies and quantitative gait analysis. Kinematics and kinetics parameters were collected for the both femora.

Results: Logistic regression analysis showed that fractured femora could be predicted with a correctness of estimation of $86.7 \%$ $(p=0.006)$. The model used 3 parameters out of 16 (foot and hip abduction moment, pelvic excursion in coronal). Sensitivity and specificity of prediction was found to be nearly equivalent.

Discussion: This study showed that the fractured femora can be estimated using gait analysis parameters. Uncorrected femoral torsion and angulation effect gait parameters but there are upward and downward compensation mechanisms to restore the significant deviations of gait characteristics. One of the compensation mechanisms of the excessive femoral torsion is open kinetic chain pronation and so foot abduction power is the most predictive value of the femoral torsion. Pelvic excursion is the main upward compensation mechanism and so it is in the model. Changes in hip abduction moment is the result of femoral ante torsion's effect on the lever arm dysfunction.

$O P-153$

Treatment of Antero Lateral Head

Dislocation Associated with Monteggia

Fracture

Lazar TODOROVIC ${ }^{1 *}$, Marjan.KAMILOSKI ${ }^{1}$, Slavica SHUPERLISKA ${ }^{1}$, Anila RACAJ ${ }^{1}$, Ljubica MIKJUNOVIKJ $^{2}$, Natalija COKLESKA ${ }^{1}$, Zllato ALEKSOVSKI $^{1}$

Authors Information:

${ }^{1}$ University Clinic of Pediatric surgery, Skopje, RN of MACEDONIA

${ }^{2}$ University Clinic of Anesthesiology and intensive care, Skopje, RN of MACEDONIA

\footnotetext{
Abstract

Introduction: Treatment of chronic radial head dislocation is still controversial. Radial head open reposition combined with ulnar osteotomy and correction is the best solution in these cases.

The aim of our study is to show the patients with overlooked antero-lateral dislocation of
}

the radius head with or without an ulnar fracture, treated at our clinic.

Material and Methods: In this study, we used cases treated from2009-2014, 9 patients with this condition were treated on the University Clinic of Pediatric Surgery in Skopje, Macedonia. Operative procedure contains open reposition of the dislocated radial head with ulnar osteotomy and annular ligament reconstruction. 4 weeks immobilization is indicated after the surgical treatment in order to achieve neutral rotation.

RESULTS: During the 24 months follow up period, clinical and radiological investigations were performed. Good radial head position was noticed in 7 patients and anterior subluxation in 2 patients. Full flexion and extension elbow movement were detected in 6 patients in our study, the pronation and supination were acceptable and valgus position was corrected.

Conclusion: After the evaluation of the presented cases, we can conclude that the open reduction and corrective ulnar osteotomy, fixed with the plate, shows good results in the overlooked antero-lateral luxation of the head of the radius with or without ulnar fracture in children.

$O P-154$

\section{Surgical Treatment of Orthopedic Shoulder Pathologies under Arthroscopic Route}

\section{Viktor MARKU ${ }^{1}$}

Authors Information

${ }^{1}$ Orthopeadic Surgeon, University Hospital of Trauma, Tirana, Albania.

\begin{abstract}
Introduction:The majority of shoulder pain problems involve the muscle, ligament, cartilage and tendons. In particular, athletes and skilled workers are especially susceptibile to issues involving shoulder blade pain or shoulder joint pain. Because of repetitive, intensive routines, shoulder pain can develop over time. This way, the patients
\end{abstract}


expect a short recovery in time from surgical treatment.

Materials and Methods: This study included 64 patients from 2015 to 2019 aged 20 to 74 years who were all undergoing surgical treatment for calcified subacromial bursa, impingement syndrome, tendon rupture, tenopathy and of scapulohumeral resection of biceps, all in arthroscopic route. In this study group, the operative time, hospital stay, rehabilitation and recovery period were monitored.

\section{Results:}

- Operator time of $40 \mathrm{~min}$ for pathologies only with calcifications of subacromial bursa and up to 1 hour 50 min of scapulo humeral luxation.

-58 cases with one day hospital stay,6 cases with two days hospital stay.

- The beginning of rehabilitation in the days before post-operative and in the second week for rotator cuff and that of scapulo-humeral luxation with the onset of limited passive movements.

- Rapid recovery from the first weeks to the 3rd month for rotary cuff and that of scapulo humeral luxation.

Conclusions: Arthroscopic minimally invasive surgical technique helps us to quickly heal patients and reduce hospital costs in short duration hospital stay.

Keywords:Shoulder, pathologies, surgical treatment.

$O P-155$

Pediatric Blunt Renal Trauma: A Single Institution 20 Years' Experience

\section{Kenan KARAVDIĆ}

Author Information:

Clinic for Pediatric Surgery, Clinic Center of

University Sarajevo, BOSNIE HERZEGOVINA

\begin{abstract}
Introduction:Kidney is the most common site of genitourinary trauma. $50 \%$ of all urinary injuries is kidney and is also affected in 8$12 \%$ of all blunt and penetrating trauma to abdomen. 80-90\% of renal injury is caused by blunt injury GY. Children, when compared to adults, are at a higher risk of renal injury from blunt trauma due to a variety of anatomic factors including decreased perirenal fat,
\end{abstract}

weaker abdominal muscles, and a less ossified thoracic cage. While there are strong trends toward non-operative management of blunt renal trauma, there are no explicit guidelines for high grade injuries.3-5 Organ preservation in children is always a primary goal with solid organ injury.

Material and Methods: All patients under the age of 18 who were admitted to Clinic for Pediatric surgery in Sarajevo with a diagnosis of renal trauma were retrospectively reviewed. Echo and CT were used to identify patients with a renal injury. The time period examined was between January 1, 19992019. Inclusion criteria were either a diagnosis of renal trauma or a diagnosis of blunt abdominal trauma and hematuria. Exclusion criterion was death due to an additional traumatic injury.

Result:Demographics include male to female ratio of 13:6 and the average age of patients was $11.9+4.6$ years. Of the nineteen patients who underwent review, eleven $(57,89 \%)$ children presented with a grade III renal injury, and eight (42,11\%) with a grade IV injury. Six patients presented with gross hematuria and 3 with microscopic hematuria. Only four patients (22\%) required blood transfusions, with the average hematocrit nadir being $31+5.3 \%$ (24.8-37.8). One of the two patients transfused had a concomitant grade IV splenic laceration with a hematocrit nadir of $24.8 \%$ and clinical symptoms consistent with shock.

Conclusions: The specificity of the child's anatomy is an aggravating prognostic factor (the kidney is larger in relation to the body cavity than in adults, less protected against the ribs, the muscles of the body and the lower abdomen, the less developed peritoneal and retroperitoneal fatty tissue).

It is recommended to initiate conservative treatment (leaching, infusion solution, monitoring) and possibly delayed surgical treatment

Indications for early surgically treatment are reserved only for patients with bleeding (absolute) and extravasation (relative)

If it is necessary surgical treatment sould be maximally preserve kidney tissue

Keywords: Kidney, pediatric, blunt, trauma 
$O P-156$

\section{Application of the Monti Procedure in Adult Continent Cutaneous Urinary Diversion.}

GezimGALIQI $^{2,}$ *, Artan $\mathrm{KONI}^{2}$, Bledar

SHEGA $^{1}$, Albert PESHA ${ }^{3}$ Luan BAJRI ${ }^{4}$

Authors Information:

${ }^{1}$ Urology Service, Regional Hospital of Shkodra, ALBANIA.

${ }^{2}$ Urology Service, American Hospital of Tirana, ALBANIA.

${ }^{3}$ Urology Service, Regional Hospital of Fieri, ALBANIA

${ }^{4}$ Anesthesi Service, Regional Hospital of Shkodra, ALBANIA.

\section{Abstract}

Introduction:Monti and co. described in 1997 a continent catheterize conduit using short segments of the ileum (1). The use of this technique allows to obtain 2 to $2.5 \mathrm{~cm}$ segment of the ileum to achieve a tabularized segment of 7-8 cm. The caliber of such a tube allows catheterization with a $16 \mathrm{~F}$ to $18 \mathrm{~F}$ catheter, and the mucosal folds of the ileum are aligned with its longitudinal axis.

Materials and Methods:From 2007 to 2019 we treated 5 patient with different condition with these procedure .First case was a middle age women with a big vesico-vaginal fistula unappropriated to close the fistula Second case a posterior urethral stricture due to pelvic fracture in a 60 years old patient unwilling to redo a urethroplasty after the failed first attempt Two cases of primary urethral tumors in womens where excision of urethra was performed with subsequent urethroplasty. The fifth one was a 20 years boy with spina bifida where an augmentation bladder procedure (2) was performed with a urinary continent Monti diversion. The patient were monitories for several years.

Results: The continence rate of our patient was good in all of them during day time and night time. All the patient where continent and achived to do a successful selfintermittent catheterisation

Conclusion:The Monti tunel is a valid option for continent intermittent catheterisation. Is an alternative to Mitrofanoff procedure. We used it when the appendix was not appropriated to use as a tunel or when it is missing. It has a good continent percentage. Of course, ours is a small serie of cases to represent more important conclusions

Keywords: Monti Procedure, Urinary Diversion, urethral stricture, vesico-vaginal fistula.

$O P-157$

Influence of Drugs on Urological Diseases

Gjergji SEMINI $^{1}$, Rustem CELAMI ${ }^{2}$

Authors Information:

${ }^{1}$ University of Ioannina, University Hospital of Ioannina, GREECE

${ }^{2}$ Professor at University of Aldent, American Hospital of Tirana, ALBANIA

\begin{abstract}
Introduction: Induced urological symptoms as side effects of prescribed treatment for various diseases.
\end{abstract}

Materials and Methods: A systematic search of the PubMed of numerous articles.

Results:Antihypertensive drugs, predominantly alpha blockers, result in stress incontinence, while selective serotonin reuptake inhibitors - SSRI, may cause urge incontinence. Commonly used combination of calcium and vitamin D supplementation for prevention of osteoporosis may be an underdiagnosed cause of renal calculi. Druginduced sexual dysfunction is a frequent side effect of antihypertensive treatment, particularly with beta adrenoceptor blockers and diuretics. The SSRI and some neuroleptics can also impair sexual function. Mesalazine for ulcerative colitis, subsequently presented recurrent renal colic due to mesalazine urinary stones. Druginduced nephrolithiasis is a rare condition in children. Drug-induced calculi represent 1$2 \%$ of all renal calculi.

Conclusions: Adverse influence of drug reactions may be hard to forecast and link to 
specific drugs and symptoms. Urological illnesses can be caused by drugs, and a careful drug history, and drug interaction filter together with timeline of symptoms will help to minimize such side effects.

KEYWORDS: Drugs, symptoms, side effects, urological, diseases.

$O P-158$

Shock Wave Lithotripsy Versus Semirigid Ureteroscopy for Proximal and Distal Ureteral Calculi less than $1,5 \mathrm{~cm}$

Rezart $\mathrm{XHANI}^{1}$, MustafaXHANI ${ }^{1}$, RudinDOMI $^{1}$, Erion DREDHA ${ }^{1}$

Author Information:

Department of Urology, University Hospital "Mother Teresa", Tirana, ALBANIA

\section{Abstract}

Introduction: We compare the efficacy of shock wave lithotripsy and ureteroscopy as well as complications related to these procedures.

Materials and Methods: This is a prospective randomized study comparing two groups of patients with a single radiopaque upper or distal ureteral stone less than $1,5 \mathrm{~cm}$ treated by ESWL and ureteroscopy between January 2014 - January 2018. ESWL was performed as an outpatient procedure using the electromagnetic lithotripter (Dornier Lithotripter S and Storz Modulith_SLX_F2 lithotripter). Ureteroscopy was performed using an $8,5 \mathrm{~F}$ semirigid ureteroscope. Intracoporeal lithotripsy with pneumatic or electro-hydraulic

energy was used when needed. An analysis was performed using four parameters (sex, stone size, degree of hydronephrosis and urinary infection). The success rates, complications, retreatment rates and auxiliary procedures were compared for each group.

Results: A total of 327 patient were treated for upper and distal ureteral stones. The success rates were $73,7 \%$ for ESWL and 88, $4 \%$ for ureteroscopy. The complication rate was higher in the ureteroscopy group (16\%) than in the ESWL group (5\%). The retreatment rate was higher in the ESWL group (57 \%) than the URS group (3\%).
Conclusions: ESWL and URS are highly effective in treatment of proximal ureteral stones less than $1,5 \mathrm{~cm}$. ESWL is safer and less invasive but URS is more effective especially in distal ureteralstones and has a lower retreatment rate.

Keywords: Shock wave lithotripsy, semirigid ureteroscopy, ureteral calculi.

$O P-159$

A Review of Dietary Treatment in Patients with Renal Stone.

Gjergji SEMINI ${ }^{1}$ Rustem CELAMI

Authors Information:

${ }^{1}$ University of Ioannina, University Hospital of Ioannina, GREECE

${ }^{2}$ Professor at University of Aldent, American Hospital of Tirana, ALBANIA

Abstract

BACKGROUND; The aim of this study was to review the studies reporting the effects of different dietary on urinary stones.

Materials and Methods: A systematic search of the Pubmed for studies, (2010 - 2015), on dietary treatment of urinary risk factors for urinary stone formation was conducted according to a methodology developed a priori. Studies were screened by titles and abstracts for eligibility.

Results:It remains a state of fact that diet has a tremendous effect in urinary stone formation, and calcium stones persist to be the most common ones, followed by acid uric stones. However, according to recent studies: in hypercalciuria; dietary calcium restriction is not recommended for stone formers with nephrolithiasis. moderate dietary salt restriction and a low-normal protein intake is useful in limiting urinary calcium excretion and thus may be helpful for primary and secondary prevention of nephrolithiasis. Omega-3 fatty acids and bran of different origin maybe help but the impact is uncertain. In hyperoxaluria: a diet low in oxalate and/or a calcium intake normal to high (800-1200 $\mathrm{mg} /$ day for adults) reduce the urinary excretion of oxalate, conversely a diet rich in oxalates and/or a diet low in calcium increase urinary oxalate. 
Conclusions:General measures: Each patient with nephrolithiasis should is the forced increase in fluid intake to achieve a daily urine output of 2 liters.

In children: Moderate dietary salt restriction and implementation of potassium intake are useful in limiting urinary calcium excretion. A balanced consumption of fruit and vegetables and a low consumption of chocolate and cola according to general nutritional guidelines.

In elderly: A restriction of sodium intake in association with a higher intake of potassium, magnesium and citrate is advisable in order to reduce urinary risk factors for stone formation but also to prevent the loss of bone mass and the incidence of hypertension.

Keywords:Diet, fluid intake, stone, urinary system.

$O P-160$

Correlation between Prostate Specific Antigen and Prostate Biopsy

\section{Herion DREDHA}

Author Information:

${ }^{I}$ Department of Urology, University Hospital Center "Mother Theresa" Tirana, ALBANIA

\footnotetext{
Abstract

Introduction:Prostate specific antigen (PSA) is a protein produced by prostate gland cells. PSA is increased in diseases such as prostatitis, hyperplasia and malignancy.

The correlation between different pathologies affecting the prostate gland and their corresponding elevation in PSA values is not constant, and exceptions may occur.

The purpose of this study is to identify the range and distribution of different prostate lesions affecting men, their association with age and evaluation of the association between total serum PSA and histological findings.

Materials and Methods:This study included a total of eighty specimens (both transurethral resection of the prostate and prostate biopsy) taken at the histopathology laboratory, in the urology service of the University Hospital Center "Mother Theresa” Tirana, Albania, for
}

a period of one year. Materials obtained for Biopsy were examined under light microscopy for final evaluation and diagnosis. All PSA values were recorded for all patients before the procedure was initiated. Statistical analysis was done using tables containing data distribution in different formats and arithmetic mean ...

Results:About $59 \%$ of the subjects studied were in the 55-70 age group. maximal PSA value ranging from 0 to $7 \mathrm{ng} / \mathrm{ml}$ benign prostatic hyperplasia was the predominant lesion $(58.75 \%)$ in the study population, while $\mathrm{P} \quad<0.01$ indicating a positive association between levels in increased PSA and chances of adenocarcinoma, results were statistically significant.

Conclusion:The results showed that the chances of finding prostate malignancy are in direct proportion to the trend of increasing PSA values, but not always a rule, but it makes the histopathologic assessment very detailed.

Keywords:Benign prostatic hyperplasia, prostate, prostate specific antigen.

$O P-161$

\section{Congenital Injuries Amniotic Band SINDROM}

\section{Arben LLOJA}

Author Information:

University Hospital Center "Mother Theresa" Tirana, ALBANIA.

\section{Abstract}

Focal ring constrictions of the extremiteties and atual loss a digit or limb are rare complication. Their gebesis is debated. Strecer (1930) and others since, maintain that localized failure of germ plasm usually is responsible for the abnormalities. Torpin (1968) and others contend that the lesions are the consequence of early rupture of the amnion which ten forms adherent tochs bands that costrict and at times actually ampute an exteremity of the fetus. Occasionally, the amputated part may be found within the uterus. 
Congenital POSTURAL DEFORMITIS: Mechanical factors arsing from chromically low volumes of amniotic fluid imposed by the small size and inappropriate of deformity, including talipes or clubfoot, scoliosiss, and hip dislocation. Hipoplastic lung also can results from oligohydramnios.

$O P-162$

Acute Kidney Injury After Major Trauma: A Sistematic Review

Matilda IMERAJ

Authors Information:

Department of Nephrology, UHC "Mother Theresa" Tirana , ALBANIA

\section{Abstract}

Acute kidney injury is a frequent complication following major trauma, it is accompanied by a high mortality rate and survivors are at increased risk for chronic kidney disease and late death .In published researches ,the incidence of post-traumatic acute kidney injury (AKI) is at a range that varies widely from 0.1 to $8.4 \%$. The causes of post-traumatic acute kidney injury (AKI) are multifactorial. The shock associated with major trauma may result in inadequate renal perfusion, rhabdomyolysis, direct kidney blunt or penetrating trauma,sepsis , hypothermia, abdominal compartment syndrome, or exposure to nephrotoxins such as contrast material andantimicrobials. Demographic patient characteristics such as male gender, older age,preexisting CKD, higher incidence rates of pre-existing comorbidities (hypertension, cardiovascular disease, diabetes, chronic obstructive pulmonary disease, metastatic cancer, hypoalbuminemia) use of (ACEI) or ARB have been implicated as predisposing to AKI .

Numerous strategies and techniques have been used from time to time for prevention of acute renal during major trauma. Dialysis is initiated for the usual indications, including volume overload, hyperkalemia, severe acidemia, and uremia, but it is possible that variations in the timing of initiation, modalities may affect clinical outcomes.In this sistematic review, we highlighted the magnitude of the problem .Efforts must be directed to improving recognition of at-risk patients, development of preventative strategies,and surveillance of long-term clinical consequences.

Keywords: Acute kidney injury , major trauma , treatment , mortality.

$O P-163$

\section{Post-Traumatic Renovascular Hypertension}

Lutfi ZYLBEARI ${ }^{1}$, Ferizate HAXHIREXHA-

DIKA, Sadi BEXHETI, Aulona HAXHIREXHA, Zamira BEXHETI, Kastriot HAXHIREXHA ${ }^{2}$

Authors Information:

${ }^{1}$ State University of Tetova, RN of MACEDONIA

${ }^{I}$ Department of Surgery, Clinical Hospital Tetova, RN of MACEDONIA.

\section{Abstract}

Introduction: There is documented evidence that after renal trauma (open or closed) after a different period of time we have arterial hypertension manifestations with renal vascular hypertension (HTARV) that is similar to that of Goldblatti who discovered in 1934 during autopsy of patients who suffered from HTA as a result of renal artery stenosis.

To verify his hypothesis, he artificially occluded the renal artery in dogs to reduce blood perfusion (apparently caused by hypoperfusion and ischemia) during which the experiment began to increase the blood pressure in the dog as a result of increased hypoperfusion. renal hypertension causing vasoconstriction and increased arterial pressure. In most cases, the mechanisms of renal hypertension are manifested as a result of traumatic perirenal hematoma, intracapsular hematoma with renal artery injury and renal parenchyma causing hypoperfusion and renal hypertension and renal hypertension.

Injury treatment was conservative (grade IIII, and surgically-grade IV-V according to the American Society for Surgery and Trauma, 1989 renal injury classification) and HTA reversal in normal condition are proofs of the concept of HTARV.

The purpose of the study: was to verify the increase in arterial pressure (unusual and rare 
cases of possible port) as a result of traumatic kidney injury.

Material and Methods: As a retrospective study material, a database of admissions to the Surgery and Intern ward was used as a retrospective study over a two-year period, identifying 10 patients, of whom 6 were males and 4 females with identical mean ages of $24.00 \pm 6.00$ years as a result of traffic trauma. Diagnosis of renal trauma is performed by screening: urine, imaging methods, glomerular filtration rate with the Tn99 DTPA and formula to the Cocrroft \& Gault formula; ultrasound, computed tomography, intravenous pyelography; Angiography.

Results: Of the total number (10 patients), 3 patients had grade IV-V injuries and were surgically treated 1 with nephrectomy and 2 days of revascularization) while 7 had grade I- kidney injury. III and were treated conservatively. All patients treated surgically had an immediate decrease in their hypertension. All patients had HTA observed after a 2-week pre-treatment period until 7-8 months. After treatment, their condition was reabsorbed and arterial pressure normalized to normal values. Only 3 patients showed the need for antihypertensive treatment with ACE inhibitors within two years which showed high positive effects and arterial pressure normalized to normal values.

Conclusion: Due to the serious complications of arterial hypertension after major renal trauma we prefer that it should be evaluated with a very serious approach and management as soon as possible by determining the degree of injury and according to the method of treatment (conservative or surgical).

Keywords: Renal trauma, renovascular hypertension.

$O P-164$

Circulating Inflammatory Biomarkers and Clinical Outcomes in COPD Caused by Biological Pathogens.

\section{Ruzhdi REXHEPI}

Author Information:

Clinical Hospital in Tetovo-Internal Disease, Tetovo, RN of MACEDONIA

\begin{abstract}
Introduction:COPD is the fourth leading cause of death worldwide. Exacerbation episodes are a hallmark of this disease. COPD exacerbation is characterized by a change in the patient's baseline dyspnea, cough, and/or sputum beyond day-to-day variability sufficient to lead to a change in medication. Infectious causes (bacteria, viruses, and atypical pathogens) and non-infectious causes (environmental exposure and poor compliance with medications) have been described. GOLD recommend antibiotics for the treatment of exacerbations.

COPD exacerbation and Systemic and airway inflammation play roles in the pathogenesis of COPD exacerbation. We hypothesized whether increased serum inflammatory biomarkers are associated with the clinical outcomes of COPD exacerbation caused by different infectious pathogens.
\end{abstract}

Materials and Methods: COPD patients with exacerbation were recruited from a Clinical Hospital in Tetovo internaldepartment during januari juli-2019. Creactive protein (CRP) were measured. Dyspnea, eosinopenia, consolidation, acidemia, and atrial fibrillation score was calculated for predicting mortality. Multiplex polymerase chain reaction was carried out for respiratory viral assay from nasopharyngeal swabs, and sputum bacterial culture was also performed, length of hospital stay were evaluated, and serum biomarkers were examined.

Results: A total of 51 COPD patients were enrolled. These patients were classified as (GOLD) stage 2, 3, and 4 in 13.2\%, 8.6\%, and $78.2 \%$ of cases, respectively. Isolated bacterial exacerbation was recovered in $30.6 \%$ of exacerbation episodes: Klebsiella pneumoniae was the most commonly identified bacteria. Viral pathogens and coinfections were noted in $9.6 \%$ and $16.1 \%$ of exacerbated patients, respectively. Influenza was the most commonly detected viral pathogen. Serum biomarkers and (Dyspnea, eosinopenia, consolidation, acidemia, and atrial fibrillation) score for viruses, bacteria, coinfection, and noninfectious causes of exacerbations were similar. Neither (Dyspnea, eosinopenia, consolidation, acidemia, and atrial fibrillation) score nor serum biomarkers were able to differentiate patients with and without 
mortality. Increased serum CRP was noted in patients with LOS $\geq 7$ days when compared with those with LOS $<7$ days $(0.38 \mathrm{ng} / \mathrm{mL}$ vs $0.1 \mathrm{ng} / \mathrm{mL} ; \mathrm{P}=0.035$ ).

Conclusion: CRP and (Dyspnea, eosinopenia, consolidation, acidemia, and atrial fibrillation) score play limited roles in predicting clinical outcome and lack an association with causes of exacerbation.

Keywords: biomarkers, inflammation, viruses, bacteria, COPD exacerbation.

$O P-165$

\section{Diagnosis and Treatment of Kidney Injuries}

Lutfi ZYLBEARI, Sadi BEXHETI, Kastriot HAXHIREXHA, Sihana AHMETI-LIKA,Aulona HAXHIREXHA, Zamira BEXHETI

Authors Information:

Medical Faculty - University of Tetova, Clinical Hospital-Tetova, PHO "VITA"-Tetove, MACEDONIA

\begin{abstract}
Introduction: Kidneys trauma represents about 1 to $10 \%$ of all intraabdominal injuries. It is estimated that more than one third of patients with polytrauma they also have renal lesions. The most common causes of this lesions are blunt injuries such as road traffic accident, powerful strokes in the lumbar region, fall from great heights, contact sports etc., while penetrating trauma of the kidneys represent only a small percentage of this injuries. In most cases kidney trauma is harmless and can be treated conservatively, so the patients do not require surgical intervention. However, in cases of major injuries, or in the case of pre-damaged kidneys a more serious treatment should be taken. One of the most characteristic signs of kidney damage is the presence of blood in the urine, whether as micro or macroscopic haematuria.
\end{abstract}

Material and Methods: in a period between January 2016 - 2019 fifty for patients have been treated at our clinic because of mild renal trauma-grade I to III according to the AAST. After admission in our department a urine sample was taken from each patient for investigation of micro or macro haematuria. At the same time a complete blood count was done. Ultrasound as a useful and accurate screening examination was undertaken in all patients. It can detect with great accuracy the kidneys hematomas and can give us accurate information on whether CT should be performed. The initial management of all patients involve basic resuscitation and dynamic monitoring of patients' general condition.

Results: from fifty for patients included in this study 35 of them was male and 19 females with an average age of 38.6 years. The most common cause of acute renal injury were traffic accidents in 39 patients, then lesions due to physical attacks in 7 patients, fall from great heights in 6 of them and accidental injuries in sports in two cases. After admission in our department laboratory investigation (urine and blood count) as well as abdominal ultrasound was done in all patients. Abdominal CT was performed in 28 patients due to suspicions of more serious injuries. According to the data from ultrasonography and CT and based on the classification of AAST scale in 34 patients the injury was classified as mild (grade I), in 18 others as a grade II, while only in two of them as a grade III. All the patients were treated conservatively which includes supportive care with bed-rest and observation along with carefully followed up of patients until macro haematuria resolves.

Conclusion: Recovery of traumatic kidney injury requires time and good monitoring of the patients until their full recovery, which can last from 2 to 6 weeks.

Keywords:trauma, kidney injury, conservatively.

$O P-166$

Exercise Induced Rhabdomyolysis with Acute Kidney Failure. A Case Report

\section{Vilma CADRI}

Authors Information:

Department of Nephrology, UHC "Mother Theresa " Tirana, ALBANIA

\begin{abstract}
Introduction: Rhabdomyolysis is a clinical syndrome caused by damage to skeletal muscle fibers with disruption and release of their contents into the circulation.
\end{abstract}


Its etiology can be highly varied. Exercise induced rhabdomyolysis is an uncommon cause of severe rhabdomyolysis and a very rare cause of acute kidney failure. We report a case of acute kidney failure following heavy exercise during exposure under extreme temperatures of $42^{\circ} \mathrm{C}$ in a healthy young adult.

Case report, a 26 years old boy was admitted at Nephrology department due to acute kidney failure. He had a three-day history of myalgia, weakness and dark color urine. During last 36 hours showed anuria.

A day before his complaints, he referred stenous exercise (raising bags of $50 \mathrm{~kg}$ ) under temperature of $42^{\circ} \mathrm{C}$ the hole day. He suffered from headaches for a year that he treated with ibuprofen $400 \mathrm{mg}$ one tablet e week.

At the time of presentation, he had urea

$94 \mathrm{mg} / \mathrm{dl}$, creatinine $9.8 \mathrm{mg} / \mathrm{dl}$, ck 3085U/l, potassium The initial treatment consisted of fluid replacement and forced diuresis.

Patient received daily sessions of dialysis for the first ten days and then three times a week for 7 days. On 28-th day he manifested prolonged polyuria for 9 days with the urine output in excess of 7 l/day at maximum. Complete recovery was observed after two months of follow up.

Conclusions: Rhabdomyolysis is not an uncommon condition. It is a life-threatening condition but a completely curable ore. Depending on severity of renal impairment some patients may need long term dialysis support.

Therefore, early diagnosis and prompt referral to appropriate center is crucial to save life.

Keywords: Rhabdomyolisis, acute kidney failure, heavy exercise, dialysis.

$O P-167$

The Efficacy of Nebulised Adrenaline in the Treatment of Children with Acute Bronchiolitis

Ferizat DIKA - HAXHIREXHA, Sevdije

KOXHA, Ledia QATIPI, Aulona HAXHIREXHA

Authors Information:

Medical Faculty - University of Tetovo, RN of MACEDONIA

PHO "Alba-Med" -Debar, RN of

MACEDONIA
General Hospital-Debar, RN of

MACEDONIA

\begin{abstract}
Introduction: Bronchiolitis is an acute disease of the lower respiratory tract characterized by obstruction of small airways. Usually It is provoked by infection of the respiratory tract from some viruses whereas the most common is respiratory syncytial virus (RSV). In the treatment of this condition, are used various drugs such as $\beta 2$ agonists and nebulised adrenaline.
\end{abstract}

Material and Methods: Forty-five children aged between one to teen yearswith first attacks of acute bronchiolitis were included in this study. All the patients were treated in our institution in a period between January 20172019. After admission and detailed examination by the paediatrician fifteen children were treated with the mixture of salbutamol $0.15 \mathrm{mg} / \mathrm{kg}$ (max. $5.00 \mathrm{mg}$ ) whereas thirty other children with adrenaline $(0.1 \mathrm{ml} / \mathrm{kg} / \mathrm{dose}$ in $1 ; 10,000)$ in $3 \mathrm{ml}$ saline solution, administered via nebuliser.

Results: According to our results the best respiratory enhancement was recorded in children after administration of adrenaline via nebuliser. The respiratory rate had fallen from 75 breaths per minute to less than 50 breaths per minute, whereas the heart rate was significantly increased. In all these children was registered a significant improvement in SpO2 - from 91 to 98. In children treated with salbutamol, the rate of respiratory improvement was lower, respectively respiratory rate had fallen from 75 breaths per minute to less than 60 breaths per minute whereas arterial $\mathrm{SpO} 2$ from 90 to the level of 96.

Conclusion:L-adrenaline is a very effective drug in improving oxygenation and support of infants with bronchiolitis in comparison with $\beta 2$ agonists.

Keywords: Adrenaline, bronchiolitis, children.

$O P-168$

C3 Glomerulonephritis in post Transplanted Patient with monoclonal gammopathy of renal significance (MGRS). A Case Report 
Arjeta DEDEJ ${ }^{1}$, Leart BERDICA ${ }^{2}$, Sotiraq LAKO $^{3}$, Fiona NASTO $^{3}$, Arjana STRAKOSHA $^{1}$

Author Information:

${ }^{1}$ Hemodialysis and Renal Transplant Service, at University Hospital Center "Mother Theresa", Tirana, ALBANIA

${ }^{2}$ Head of the Department of Anatomy-Pathology American Hospital Tirana, ALBANIA

${ }^{3}$ Hemodialysis and Renal Transplant Service, American Hospital Tirana, ALBANIA

\begin{abstract}
Introduction: C3 glomerulopathy includes several rare forms of glomerulonephritis withunderlying defects in the alternative pathway of complement cascade. It is characterized by predominant C3 deposition in glomeruli due to abnormal activation of the alternative pathway of complement system. C3 GN has been reported to be associated with several systemic diseases
\end{abstract}

Material \& Methods; We will describe a case presenting C3 GN in a patient with monoclonalgammopathy of renal significance (MGRS)

Results:A 61 years old man patient presented with gross hematuria, anemia, renal disfunction (creatinemia 2,4 mg/dl), proteinuria $814 \mathrm{mg} / 24 \mathrm{~h}$. The patient was transplanted two years ago and he was taking corticosteroids, MMF, tacrolimus and entecavir for hepatitis B. Serum protein electrophoresis; hypogammaglobulinemia with a small homogeneous spike-like peak. Serum Kappa free light chains 32,4 mg/dl, serum Lambda free light chains $9.3 \mathrm{mg} / \mathrm{dl}$. Ratio 3,4. Autoimmune tests ANA, ANCA, Anti ds DNA C3 and C4 were negative. Urine Kappa Light Chains 26,4 mg/24 h, urine Lambda Light Chains 6,6 mg/24h. Urine Kappa/Lambda ratio 4. After consultation with hematologist results of bone narrow biopsy came for monoclonal gammopathy, and FISH conclusion is presence of $t(11,14)$ (q13, q32) which originated from IgH / CCND1 retraction and 1q21 acquisition. Renal biopsy is C3 glomerulopathy with mesengial and diffuse endocapillary proliferation under light microscope and diffuse deposition of C3 and no immunoglobulin under immunofluorescence microscope.
Conclusions: Monoclonal gammopathy of renal significance MGRS is a term to describe a group of hematological disorders associated with kidney disease that fail to meet the standard definitions for MM or lymphoma. In such cases, the renal impairment is often linked to the underlying hematological disorder. The intention was to make a clear distinction between MGUS, a benign asymptomatic condition, and MGRS, which may be associated with significant morbidity and mortality.

Keywords: C3 glomerulopathy, complement, MGRS, renal disfunction

$O P-169$

\section{Influencing Factors in the Evolution of Renal Transplant Patients.}

Arjana STRAKOSHA ${ }^{1}$, Nevi PASKO ${ }^{1}$, Vilma $\mathrm{CADRI}^{1}$, Fiona NASTO ${ }^{2}$, Arjeta DEDEJ ${ }^{2}$, Nestor THERESKA ${ }^{2}$, Myftar BARBULLUSHI ${ }^{1}$.

Author Information:

${ }^{1}$ Hemodialysis and Renal Transplant Service, at University Hospital Center "Mother Theresa", Tirana, ALBANIA

${ }^{2}$ Hemodialysis and Renal Transplant Service, American Hospital Tirana, ALBANIA

\begin{abstract}
Introduction: Currently kidney transplantation is the treatment of choice for patients with Chronic Renal Disease in advanced stage when GFR $<15 \mathrm{ml} / \mathrm{min} / 1.73$ sq.m2. Despite new treatment regimens with immunosuppressive drugs and new supportive therapies, the task of the clinician following these patients remains difficult due to the high morbidity and the increasing number of concomitant diseases. Also in recent years new medications and treatment schemes have been associated with new complications that may affect the transplanted organ recipient by significantly altering their outcomes and clinic.
\end{abstract}

Our study through multivariable analysis identified some of the major factors affecting graft and patient life expectancy such as dyslipidemia, hypertension, proteinuria, anemia, but all of these factors combined with donor and recipient age, with hemodialysis pre-transplant duration., from severe or mild 
infections passed on by recipients, like many of these factors we cannot disconnect from treatment with immunosuppressive drugs that make these risk factors more influential.

In this respect, this study is of value because in this complex situation of combining many influential factors, we may intervene in some of them to improve the prognosis of the graft and recipient.

Keywords: kidney transplantation, immunosuppressive, supportive therapies

$O P-170$

\section{An Albanian Experience in Laparoscopic Treatment of Esophageal Achalasia}

\section{Arben BEQIRI}

Author Information:

General Surgeon, University Hospital Center "Mother Theresa", Tirana, ALBANIA

\begin{abstract}
Introduction:Achalasia is a rare primary motility disorder of the esophagus that affects one person in 100,000 per year and is characterized by the absence of esophageal peristalsis and incomplete relaxation of a frequently hypertensive lower esophageal sphincter (LES) in response to swallowing. Treatment of the pathology include; Nonsurgical 1. Medicaments such as Nifedipine and Nitrates, 2. Endoscopy (Balloon dilatation, use of botulinum toxin type A) and the most innovated POEM and 3. surgical methods (Heller myotomy with Door Fundoplication). Heller myotomy with fundoplication is considered worldwide the golden standard for the treatment of patients with achalasia.
\end{abstract}

Materials and Methods:A retrospective survey was conducted between 2016-2019, including 19 cases diagnosed with: Esophageal achalasia who have undergone the procedure: Extra-mucosal Heller cardiomyotomy with Door Fundoplication in laparoscopy (15 cases were performed in the American Hospital and 4 cases were performed in University Hospital center "Mother Theresa" Tirana, Albania.)

Results:Related to the surgery procedure, only in 2 cases, during the interventions with Heller myotomy in laparoscopy, a perforation of the esophageal mucosa occurred. In one case was proceeded with the resection of the distal esophagus because of the long stenosis of the lower esophagus, after previous Heller myotomy performed 20 years before the presentation. All the cases had a good post operative recovery. On the $3^{\text {rd }}$. day of hospitalization, patients underwent radiographic studies with oral digestion of contrast (Barium), following a specific diet after the procedure and follow up (in $1,3,6$ months) in the subsequent months showing improvement not only clinically but also in radiologic studies.

Conclusion:Heller Cardio myotomy with Door Fundoplication resulted in various clinical improvements; Reducing gastro esophageal reflux (GERD) and dissolving dysphagia (Major part of the patients had dysphagia for more than 5 years). There are significant results in reducing the lumen of dilated esophagus (in the radiographic studies obtained months later). Laparoscopic approach for esophageal achalasia is the most effective surgical technique that provides both short and long-term symptomatic relief with released hospitalization time and less complication rate. It is the intervention of choice especially in patients who have not responded to other methods, like prior endoscopic interventions.

Keywords: Achalasia, Heller, laparoscopy.

$O P-171$

Tips and Tricks for Minimally Invasive
Management of Acute Intestinal
Obstruction. Nuhi ARSLANI ${ }^{1}$, Basri LENJANI ${ }^{1}$, Rok KOLARIĆ ${ }^{2}$

Authors Information:

${ }^{1}$ Department of Abdominal and General Surgery, University Clinical Center, Maribor, SLOVENIA

${ }^{2}$ Faculty of Medicine, University of Maribor, SLOVENIA

\begin{abstract}
INTRODUCTION: Main limitations of the laparoscopic procedure are visualization of the abdominal cavity and complications related to blind placements of laparoscopic tools. There are few selection criteria to be used for laparoscopic treatment of intestinal
\end{abstract}


obstruction such as suspicion of partial intestinal obstruction and suspicion of proximal intestinal obstruction. Some clinical parameters can predict the success of laparoscopic treatment, including improvement after nasogastric tube placement and no fixation of obstructed bowel segments to the retroperitoneum. Emergency surgeries are done without prior staging work up and therefore hold great risk for mortality. Selfexpandable metallic stents (SEMS) provide time for preoperative evaluation of the patient's condition and allow the possibility of patient's medical condition to improve.

Materials and Methods: 3 patients who underwent minimally invasive treatment of acute intestinal obstruction are presented; "patient 1" with acute small-bowel obstruction due adhesions who underwent diagnostic laparoscopy, "patient 2" acute small-bowel obstruction due to intussusception and "patient 3" with acute large-bowel obstruction due to carcinoma of sigmoid colon who have had SEMS placed.

Results:In patient 1, diagnostic laparoscopy eliminated mechanical obstruction as a cause of symptoms and adhesiolysis was performed. In patient 2, diagnostic laparoscopy led to finding of two invaginations at jejunoileal transition which were desinvaginated during the procedure. In patient 3, colonic stent was positioned in patient's sigmoid colon which relieved obstruction due to luminal stenosis.

Conclusion:Minimally invasive operative treatment of intestinal obstruction is chosen according to the discussed selection criteria, where indications, contraindications, advantages and limitations of such procedure are carefully considered.

Keywords:intestinal obstruction, laparoscopy, exploration, desinvagination, stenting.

$O P-172$

Treatment of Morbid Obesity with Laparoscopic Sleeve Gastrectomy

Gazmend ELEZI ${ }^{{ }^{*}}$, Lindita SEJFULLAHUELEZI $^{1}$, Merita RUSTEMI-ELEZI ${ }^{1}$, Burim ELEZI $^{1}$

Authors Information:

${ }^{1}$ City General Hospital $8^{\text {th }}$ September-Skopje, Republic of North MACEDONIA

\section{Abstract \\ Introduction:}

TheepidemicofobesityintheWorldis a major public health issue and more than a third of adults are now considered obese (body mass index $>$ or $=30 \mathrm{~kg} / \mathrm{m}^{2}$ ). Surgery for morbid obesity, bariatric surgery, is the most durable treatment for this disease.

Primary bariatric surgery has been proven to be effective in weight loss and improvement of weight-related metabolic co-morbidities.

Materials and Methods: Thisconstitutes a prospectivestudycarriedoutin a tertiarycareCityHospitalinSkopje $\quad$ " 8 th September" and included 8 morbidly obese patients who underwent LSG. The operation was performed through one 15, one $12 \mathrm{~mm}$ and two $5 \mathrm{~mm}$ ports, using the Endo-GIA stapler to create a lesser curvegastrictubeover a 36-Fr bougie.

Results:Operativetime, complicationrates, hospitallengthof stay, Body Mass Index (BMI), \% of Excess Weight Loss (EWL) and appetite were evaluated. There were 3 females and 5 males, aged (mean+/-SD) $35.5+/-10.5$ yrs and preoperative BMI 47.8+/$7.5 \mathrm{~kg} / \mathrm{m} 2$. Three of them had Diabetes mellitus type $2(8 \mathrm{mmol} / \mathrm{L}+/-3 \mathrm{mmol} / \mathrm{L})$, Sleep apnea all of the patients, Hypertension four of them $(140 / 90 \mathrm{mmHg}+/-20 / 15$ $\mathrm{mmHg}$ ). The operative time was $110.7+/-30$ min.

\section{Conclusions:}

Althoughthenumberofpatientsisrelatively small, the data of this study indicate that laparoscopic sleeve gastrectomy is effective in weight reduction, normalization of diabetes mellitus type 2, loss of sleep apnea and normalization of blood hypertension. A higher number of patients and longer followup period will be necessary to evaluate longterm efficacy.

Keyword: Morbid, obesity, laparoscopic sleeve gastronomy.

$O P-173$

Non- Surgical Weight Loss Procedures
(Gatsric Batox, Intragastric Balloon)

Selim Birol

Author Information:

General Surgery, Maltepe C.I.K State Hospital, Istanbul, TURKEY 


\begin{abstract}
Obesity is an epidemic worldwide and its incidence is continuing to grow up. Today more people die from obesity and its results than from hunger in the world. Being number one preventible death cause, obesity deserves to be prevented or treated.

Else than certain steps and measures to prevent obesity from begining, which actually should be another subject, there are many options for its treatment as well. Medical therapy, endoscopic bariatric interventions and bariatric surgery are the main treatment options.

Bariatric surgery has its well-known specific risks which may prevent the patient from surgery and direct him or her to non-surgical treatments. Also there is another group of patients who are overweight or obese without meeting the criteria of bariatric surgery.

No matter what the reason is, there are and will be an important number of patients who would seek for non-surgical weight loss procedures. Using Type A Botulinum Toxin for weight loss is relatively new method.

My talk will include the technic of endoscopic gastric botulinum toxin injection, possible adverse effects and the results according to our experience.
\end{abstract}

$O P-174$

Bariatric and Metabolic Surgery (Laparoscopic Vertical Sleeve Gastrectomy, Transit Bipartition, Ileal Interposition)

\section{Dr. Hasan Vedat OFLUOĞLU}

Author Information:

General Surgery, Maltepe C.I.K State Hospital, Istanbul, Turkey

\footnotetext{
Abstract

The fast increase in the incidence of obesity makes it an almost worldwide epidemic. The health risks caused by obesity alone or as being a major component of the "Metabolic Syndrome" are mainly Type 2 Diabetes, Cardio-vascular events, Stroke, Articular problems, PCOS, Hypertension and Sociopsychologic problems. As well as being a major health treat, obesity and its results bring a considerable burden to the economies.
}

Manegement of obesity includes medical, endoscopic and surgical interventions.

Laparoscopic Vertical Sleeve Gastrectomy is considered as the primary or the first step surgical intervention to those with morbid obesity or heavier stage. Thanks to the advances in stapling technology and vascular energy devices, Laparoscopic Sleeve Gastrectomy has become a very effective and safe surgical treatment for obesity today.

Approach to an obesity patient, the decision for Laparoscopic Sleeve Gastrectomy (LSG), the steps to prevent complication and the the technic will be the main topics in my talk.

$O P-175$

\section{Standardized Laparoscopic Sphincter preserving total mesorectal excision for rectal Cancer}

\section{Burak KOZA}

Authors Information:

${ }^{1}$ General Surgery Department, American Hospital, Tirana ALBANIA
Abstract
Introduction:Current laparoscopy surgeries are not new in the treatment of rectal cancer in everyday practice, our goal is to describe a standard surgical technique of laparoscopic interventions in this region.
There are many descriptions of the surgical technique used for, total mesorectal excision laparoscopic as well as manual assistance, direct laparoscopy, hybrid laparoscopy including sphincter-saving procedure and abdominoperineal resection. The efficacy of laparoscopy for rectal cancer is still controversial due to the lack of data regarding long-term oncologic outcomes, patient selection bias, and technique of use. This study was designed to evaluate the impact of a total mesorectal excision standard laparoscopic sphincter preservation technique for rectal cancer neoplasia on the long-term outcome of an unselected cohort of patients. This was a prospective study, and data were extracted from a prospectively maintained database. Consecutive patients with histologically proven rectal cancer underwent total mesorectal excision standard laparoscopic surgery. 
Patients with previous conventional abdominal surgery defined as other than cholecystectomy or appendectomy and patients with morbid obesity defined as body mass index, tumors... were not excluded. The conclusion of the study was the long-term oncological outcome after a total mesorectal excision standard laparoscopic technique. The long-term oncological outcome includes the following variables: operative time, distal and lateral removal margins,

KEYWORDS: laparoscopy, total mesorectal excision, rectal cancer

$O P-176$

\section{Laparoscopic Inguinal Hernia Surgery (TEP - Sils TEP)}

Selim BIROL

Author Information:

General Surgery, Maltepe C.I.K State Hospital, Istanbul, TURKEY

\section{Abstract.}

Laparoscopic inguinal hernia repair originated in the early 1990s as laparoscopy gained a foothold in general surgery. Inguinal hernias account for $75 \%$ of all abdominal wall hernias, and with a lifetime risk of $27 \%$ in men and 3\% in women. Repair of these hernias is one of the most commonly performed surgical procedures in the world laparoscopic hernia repair is similar to other laparoscopic procedures. General anesthesia is given, and a small cut (incision) is made in or just below the navel. The abdomen is inflated with air so that the surgeon can see the abdominal (belly) organs.

A thin, lighted scope called a laparoscope is inserted through the incision. The instruments to repair the hernia are inserted through other small incisions in the lower abdomen. Mesh is then placed over the defect to reinforce the belly wall.

Laparoscopic inguinal hernia repair has become a valid option for repair of an inguinal hernia, although the primary indication for the use of laparoscopic inguinal hernia repairs has been for bilateral and recurrent inguinal hernias. As more experience has been gained with the laparoscopic techniques, it is now used for the repair of the primary/unilateral inguinal herniaPotential benefits of the laparoscopic approach include quicker postoperative recovery and possible decreased incidence of long-term groin pain

OP - 177

Dislocation of Total Hip Arthroplasty, Current Concepts Review

Ledian FEZOLLARI ${ }^{1 *}$, Ilir HASMUCA ${ }^{1}$, Alban HYSENI $^{1}$, Arsel DIZDARI ${ }^{1}$, Ylber ZAMA ${ }^{1}$, Armand STAFUKA ${ }^{1}$, Gjergj CAUSHI ${ }^{1}$, Vilson RUCI $^{1}$, Artid DUNI ${ }^{1}$, Ergys CAMI ${ }^{1}$, Alfred GEGA $^{1}$, Gezim BIBA ${ }^{1}$, Onder DELIALIOGLU ${ }^{2}$, Kenan BAYRAKCI ${ }^{2}$, Guido BASSI ${ }^{2}$

Authors Information:

${ }^{1}$ Orthopedic Service, University Hospital of

Trauma, Tirana, ALBANIA-

${ }^{2}$ Orthopedic Service, American Hospital Tirana, ALBANIA

Abstract

Most of dislocations occur within the first 3 months following surgery $50 \%-70 \%$ of dislocations occur within the first 5 week to 3 months postoperatively, and more than $3 / 4$ th of dislocations occur within the first year following surgery.

The cumulative risk of dislocation does not remain constant following THA, increasing with time due to trauma, polyethylene wear, increased pseudocapsule laxity and deteriorating muscle strength. The cumulative risk of dislocation within the first postoperative month is $1 \%$ and within the first year approximately $2 \%$. Thereafter, the cumulative risk continuously increases by approximately $1 \%$ per 5 -year period and amounts to approximately $7 \%$ after 25 years.Late dislocations are caused by progressive improvement in motion after surgery

Late dislocations are more likely to become recurrent and require surgical intervention.

Among all causes of revision hip surgery, dislocation is the most frequent, accounting for $22 \%$ of total hip revisions in US Medicare 
data.Dislocation is also a relatively common complication, occurring in approximately $0.3 \%$ to $10 \%$ of all primary procedures.

Understanding the causes of hip dislocation can help the surgeon to identify factors related to the patient, the implant, and surgical technique which contributes to this complication.

With this understanding, most dislocations can be avoided.

$O P-178$

\section{Periprosthetic Fractures after Total Knee Replacement}

AntonioGAVRILOVSKI ${ }^{l}$, RezeartDALIPI ${ }^{1 *}$, AleksandarTRAJANOVSKI ${ }^{l}$, Aleksandar SAVESKI $^{l}$

Authors Information:

${ }^{1}$ Orthopaedic Surgeon University Clinic for TOARILUC, - Skopje, RN of MACEDONIA

\begin{abstract}
Total knee replacement is one of the most common procedures in the today's orthopedic surgery. With the annual increasing number of total knee replacements done in the world, there is an increase in the number of complications.

Periprosthetic fractures are one of the most difficult and challenging complications in orthopedic practice even for the most experienced surgeons.

There are few proposals for classification systems and treatment algorithms but still there are no gold standard guidelines.

In our clinic annually around 100 total knees are implanted.

We present our experience in the treatment of these fractures in the period 2009-2019.
\end{abstract}

Keywords: Knee, fracture, surgery, orthopaedic.

$O P-179$

Inserting Distal Screws into Interlocking Nails without Image Intensifier
Murat Onder DELIALIOGLU ${ }^{1}$, Ledian FEZOLLARI $^{2}$, Kenan BAYRAKCI ${ }^{1}$

Authors Information:

${ }^{1}$ Orthopedic Services, American Hospital, Tirana, ALBANIA.

${ }^{2}$ Department of Orthopedic, University Hospital of Trauma Tirana, ALBANIA.

\begin{abstract}
Introduction:Accurate placement of the distal screws into the interlocking nails is a demanding procedure secondary to inaccuracy of the targeting device supplied with the system and to deformation of the nail during insertion. Distal locking can be time consuming and expose the patient and operation team to unnecessary increased ionizing radiation. We describe a new protocol for the insertion of the distal screws into femoral and tibial interlocking nails by using only another same length nail without an image intensifier.
\end{abstract}

Material and Methods:Twenty patients (eight tibiae and 12 femora), among those attended to our institution during the last three years treated with reamed, locked intramedullary nailing were included. After placement of the nail in a tibia or femur, a second interlocking nail at the same length is placed along the surgical limb. Using the proximal insertion jig provided with the system, proximal holes of the internal nail are locked with long screws passing through the nail-mounted outrigger to the far cortex. After avoiding the outrigger motion of the external nail, distal screws are locked successfully in all cases.

Results:In 20 consecutive distally locked nailing, the technique was easily mastered, helped to decrease the roentgenogram exposure and minimize the operating time when compared to freehand techniques.

Conclusions:This technique can be a safe alternative to the common distal targeting techniques. It is simple, safe, universal, reproducible, rapid and inexpensive, avoiding the use of distal targeting guides. It can be used without an image intensifier.

Keywords;interlocking nails, distal screws, image intensifier 
$O P-180$

\section{Torsional Deformity and Foot Progression angle following Pediatric Femoral Shaft Fractures.}

Kenan BAYRAKCI ${ }^{1,}$ Onder M.DELIALIOGLU ${ }^{1}$, Ledian FEZOLLARI ${ }^{2}$

Authors Information:

${ }^{1}$ Orthopedic Services, American Hospital, Tirana, ALBANIA.

${ }^{2}$ Department of Orthopedic, University Hospital of Trauma Tirana, ALBANIA.

\begin{abstract}
Introduction:Our study aimed to determine the frequency and degree of torsional deformity following femoral shaft fractures to relate them to clinical complaints using foot progression angles (FPA) obtained from gait analysis.
\end{abstract}

Material and Methods: We examined both femora of seventeen children admitted to our hospital for unilateral femoral fracture in fourteen, and bilateral in three. Nine were male and eight were female. The average age was 9.4 years, ranging from 5years to 15 years. The cases were followed up with an average period of 23 months (6-48 months).

We evaluated each patient with computerized gait analysis and computerized tomography. Our premise was that foot progression angle may give insight about how subjects with uncorrected femoral antetorsion adapt.

Results: The mean femoral torsions for injured and sound side femora were $6.88^{\circ} \pm$ $18.21^{\circ}$ (mean \pm SD, range $-20^{\circ}-44^{\circ}$ ) and $11.28^{\circ}$ $\pm 16.57\left(-20^{\circ}-38^{\circ}\right)$ respectively. The mean foot progression angles for injured and sound side were $12.85^{\circ} \pm 5.72^{\circ}\left(4.20^{\circ}-26.40^{\circ}\right)$ and $12.27^{\circ} \pm 3.98\left(3.80^{\circ}-18.90^{\circ}\right)$ respectively

The Pearson correlation coefficient of the relation was $0.454 \quad(p=0.044)$ between femoral torsion value of the injured and FPA of the sound side and $0.465 \quad(p=0.039)$ between femoral torsion value of the sound and FPA of the injured side.

Conclusion: None of the cases and their parents had complaint. We observed femoral torsion differences of up to $40.3^{\circ}$ without any clinical symptoms. It has been seen that the mean difference of FPA was $0.48^{\circ}$ despite high femoral torsion differences.

Keywords:femoral shaft fractures, femoral torsions, foot progression angles.

$O P-181$

\section{Patella Fractures and their Treatment (Retrospective Study)}

\section{Alban HYSENAJ}

Author Information:

Orthopedic Resident, University Hospital Center "Mother Theresa", Tirana, ALBANIA
Abstract
A patellar fracture is a serious injury that can make it difficult or even impossible to straighten your knee or walk. Some simple patellar fractures can be treated by wearing a cast or splint until the bone heals. In most patellar fractures, however, the pieces of bone move out of place when the injury occurs. Fractures make up about $1 \%$ of all fractures. Patella plays an important role in knee extension.
Depending upon your specific fracture, you may be allowed to bear weight on your leg while wearing a cast or brace. With some fractures, however, weight bearing is not allowed for 6 to 8 weeks. Your doctor will talk with you about restrictions on weight bearing.
Study of four-year prospective fracture from 2015 - August 2019, at University Hospital of Trauma, study of fracture patterns, correlation with injury mechanism and age. Study the whole method of surgical treatment.
How long it takes to recover after a patellar fracture will depend upon a number of factors, including: The severity of your injury; Whether your treatment was surgical or nonsurgical; The time needed for rehabilitationMost patients will be able to return to their normal activities within 3 to 6 months. For patients with severe fractures, the return to activity may take longer. Your doctor may suggest some lifestyle changes to help protect your knee and prevent future problems. This may include avoiding activities that involve repetitive deep knee bending or squatting. 
Climbing stairs or ladders should beavoided, as well.

Keywords:Fracture, patella, extension, surgery.

$O P-182$

The Influence of Damage Control Orthopedic Surgery in the Polytraumatic Patient

Dritan TODHE ${ }^{1,2}$, Blerta HAMOLLI ${ }^{2}$, Alban HYSENAJ $^{2}$, Rezart ÇIPI ${ }^{2}$

Authors Information:

${ }^{1}$ Hygeia Hospital Tirana, ALBANIA

${ }^{2}$ University Hospital of Trauma, Tirana, ALBANIA.

\begin{abstract}
Introduction:Damage control orthopedics is a relatively new concept and it occupies a small proportion of the overall process in a polytraumatic patient. It necessitates continuous communication between surgical, anesthetic and the whole multidisciplinary team. Our randomized study in a first-level Trauma Center showed a benefit for borderline patients treated by DCO in comparison to early total care. The damage control strategy has been adopted by traumatologists to treat extremity fractures.
\end{abstract}

Material \& Methods:For the study we considered 33 patients (19 Males and 14 Females) from 21 to 50 years old with severe polytraumatic damages, including even orthopedic injuries that were managed with DCO during the period of 6 months (from January 2018 to June 2018) in University Hospital of Trauma, Tirana. 9 of 33 patients were send to the ICU. The other patients were subject of surgery in terms of DCO, that consisted on: Immediate life-saving surgery, which was required in 21 cases; Surgery for controlling heavy bleeding, which was required in 7 cases;Wound bleeding control, infection control, washing, dressing, and temporally closing the wound; and Provisional minimally-invasive external fixation, which was performed in 5 patients.

Results:The study demonstrated the enormous time savings and reduction of blood loss during initial treatment if patients with multiple injuries are treated according to DCO. We feel that DCO is a safe strategy in severely injured patients with multiple injuries who are too critically ill for definitive surgery and for those patients who cannot be safely assigned for early total care treatment.

CONCLUSIONS: The method was adapted to gain control of an unstable situation until definitive intervention could be undertaken.

KEYWORD: Damage control, orthopedics, Surgery, extremity fractures

$O P-183$

Benign Bone Tumors, a Descriptive Study in 10 Years.

Blerta HAMOLLI ${ }^{1}$, Arnel MICI ${ }^{1}$, Sokrat BERDUFI ${ }^{1}$

Authors Information:

${ }^{1}$ Orthopedic Service, University Hospital of Trauma, Tirana, ALBANIA

Abstract

Introduction:Benign bone tumors are benign lesions that compromise the quality of life of the affected patients. As they are more common in younger people and they are mostly asymptomatic lesions, the importance given to diagnose and treat them is vital to the potential morbidity (potential fractures) that these lesions carry. By correctly establishing the diagnosis with the relevant histological typology, we can prevent aggravation of the affected bone structure, regardless of anatomical location. The purpose of this study is to show that diagnostic orientation leads to efficient surgical treatment and reduction in the number of patients presenting with relapses.

Material and Methods:This is a study based on 292 cases (from 1700 bone biopsies) and their histopathological examination data presented not only at Trauma University Hospital but also at other hospitals, thus having a heterogeneity in the distribution of variables such as age, sex and anatomical locations. It is a retrospective study of both diagnosis and treatment. The evaluation was performed on the basis of clinical, radiological and histological criteria. 
Results: The study found that the most common benign bone tumor was

Osteochondroma (39.6\%), Aneurysmal Cyst (12.6\%) Simple Cyst (13.8\%) TGJC (21.2\%) Osteoid Osteoma (8.9\%) Male Female Ratio male predominance. These tumors most affect children and adolescents (29.9 \pm 15.7$)$. Most affected bone: Femoral (87 cases) and 6\% of cases had relapses. Most patients come from urban areas. Clinical and histopathological framework not always consistent with significant $\mathrm{p}<0.001$.

Keywords:Tumor, bone, histopathological examination.

$O P-184$

Ruptured and Infected Pseudoaneurysms of the Femoral Artery in a Drug Addict

Albana KENGA ${ }^{1}$, Ervin BEJKO ${ }^{2}$, Frenki

VILA $^{3}$; Sokol XHEPA ${ }^{1}$

Authors Information:

${ }^{I}$ Service Of Vascular Surgeon, University Hospital Center "Mother Theresa" Of Tirana, ALBANIA

${ }^{2}$ Anesthesiologist, University Hospital Center "Mother Theresa" Of Tirana, ALBANIA

3 Resident Of General Surgery, University Hospital Center "Mother Theresa" Of Tirana, ALBANIA

\begin{abstract}
Introduction:FAPs in $\mathrm{I} / \mathrm{V}$ drug users are usually caused by punctures of the femoral artery. Iatrogenic pseudoaneurysms (IPA) form when an arterial puncture site fails to seal, allowing arterial blood to jet into the surrounding tissues and form a pulsatile hematoma. These lesions lack a fibrous wall and are contained by a surrounding shell of hematoma and the overlying soft tissues. It can present as a new thrill or bruit, pulsatile hematoma, or marked pain or tenderness. Complications of pseudoaneurysms include rupture, distal embolization, local pain, neuropathy and local skin ischemia.
\end{abstract}

We report a rare case of 26-year-old male with an infected femoral pseudoaneurysm of the left leg, presented with a 10-day history with mild pain and tenderness of the lower left extremity, in the inguinofemoral area. The patient is known to have a history of I.V drug abuse. The IPA ruptured while the patient was being prepared for surgery. Due to its location, the patients clinical state, physical parameters and anatomical state of the damaged vessel, emergency surgery was required. As the reconstruction of the common femoral artery was not an option, an external iliac - superficial femoral artery bypass was performed. The presentation, the diagnostic evaluation, and the surgical management of the aneurysm are further discussed in our presentation. The patient had an optimal postoperative course and a 10-day hospital stay.

Discussions: A high rate of limb loss occurs and many patients with retained lower extremities suffer unreported incidences of residual claudication or rest pain, resulting from pseudoaneurysm resection and sole artery ligation. There is no level I evidence to support that ligation alone; excision of the PA and eradication of local sepsis is the best methods of treatment of infected pseudoaneurysms from substance abuse. The mandate is to devise treatment that reduces the rate of amputation and the incidence of chronic ischemic symptoms, with a low risk of graft infection.

Keywords:Femoral artery, pseudoaneurysm, rupture, infected.

$O P-185$

Retroperitoneal Hematoma Related to Spontaneous Rupture of Thrombosed Iliac Vein. - Two Cases and Review of Literature.

Gentian CACO ${ }^{1,2}$, Petrika GJERGO², Lordian NUNCI ${ }^{3}$.

Authors Information:

${ }^{1}$ Vascular Surgeon UHC "Mother Theresa", Tirana, ALBANIA. 
${ }^{2}$ Service of Vascular surgery, UHC "Mother Theresa", Tirana, ALBANIA.

${ }^{3}$ Central Intensive Care Unit, UHC "Mother Theresa”, Tirana, ALBANIA.

\begin{abstract}
Introduction: Spontaneous rupture of thrombosed iliac vein is a rare event with only a few reported cases. It may be life threatening because of the combination of both thrombosis and hemorrhage, two situations whose treatment seems contradictory. We report two cases diagnosed and treated in our hospital and we also make a review of the literature.
\end{abstract}

Case Presentation: Two women,58 and 65 years old respectively, presented in emergency with collapse and acute swelling of left lower limb. Both patients had no important previous medical history. They had complained acute lumbar or abdominal pain at the very beginning of their illness. Severe anemia and signs of hemorrhagic shock were present at presentation. CT scan showed retroperitoneal hematoma on the left side and occlusive thrombus of ipsilateral iliac veins on both cases.

The first case was operated at emergency. After retroperitoneal clot removal a rupture of the lateral wall of the thrombosed common iliac vein was found. The vein was legated and the patient treated with anticoagulation.

The second case had been considered pulmonary embolism at presentation and no CT scan of abdomen had been performed because of severe hemodynamic instability. She was treated conservatively with repeated blood transfusions and anticoagulation at low doses. The next morning a CT scan was performed and diagnose confirmed. Since the patient was stable conservative treatment continued.Both patients had slow but good recovery. They were treated with long term anticoagulation.A search for the condition at Pubmed revealed 38 publications with 46 patients. The majority were women over 60 years of age with left leg vein thrombosis.
There were different treatment strategies, surgical, endovascular and conservative.

Conclusion:Rupture of thrombosed iliac vein is a rare complication of deep vein thrombosis. Its presentation is dramatic. Clinical suspicion and CT scan should lead fast diagnose and treatment to lower the high mortality rate of such a complication.

Keywords:Retroperitoneal Hematoma, rupture, vein thrombosis.

$O P-186$

A Very Rare Complication of an Abdominal Aortic Aneurysm:Primary

Aortoduodenal Fistula without

Gastrointestinal Bleeding

Sokol XHEPA ${ }^{1 *}$, Ervin BEJKO², Denis KOSOVRASTI $^{1}$, Marsela SOPIQOTI ${ }^{1}$, Stavri LLAZO $^{2}$; Astrit XHEMALI ${ }^{3}$, Frenki VILA ${ }^{3}$, Eljona XHELILI ${ }^{3}$

Authors Information:

${ }^{1 *}$ Service of Angiology \& Vascular Surgery UHC "Mother Teresa" of Tirana, ALBANIA

2. Service of Anesthesiology \& Intensive Care UHC "Mother Teresa" of Tirana, ALBANIA

3. Service of General Surgery University Hospital Center "Mother Teresa" of Tirana, ALBANIA

\begin{abstract}
Introduction: To report the management of a very rare combination, Abdominal Aortic Aneurysm (AAA) with Duodenal Fistula, but without upper gastroduodenal bleeding, because a massive wall aortic thrombus kept closed the communication between aortic lumen and duodenum.

Case Report:A 84-year-old man was presented at the Service of Emergencies at UHC "Mother Teresa" of Tirana on August 21, 2018, with epigastric and back pain and without anemia.There was no history of analgesic abuse, peptic ulceration, alcohol excess, weight loss or gastrointestinal bleeding. The abdomen was soft and below the epigastric region was a pulsatile mass. A computed tomography detected a $6.5 \times 10$ $\mathrm{cm}$ abdominal aortic aneurysm with a massive anterior mural thrombus and no signs of a leak. Inside of the right side of the mural thrombus was seen air, which was a suspicion for an aortoenteric fistula.
\end{abstract}


This suspicion led to immediate surgical exploration and an aortoduodenal fistula was confirmed. Both, the aorta and the duodenum were successfully repaired by aortobiiliac synthetic graft replacement and duodenostomy, respectively.

The patient made an uneventful recovery. He remains well and 17 days later he left hospital with the duodenostomy catheter closed and normal oral nutrition.

Conclusions:Primary aorto duodenal fistula without gastrointestinal bleeding is a very rare and "lucky" complication because the patient can be operated without severe anemia and hypovolemic shock. The strategy of treatment includes: fast diagnosis, immediate surgical treatment and the simplest possible surgical reconstructions for both pathologies. Keywords:AAA, aorto-duodenal fistula, aorto biiliac graft, duodenostomy.

$O P-187$

Neurovascular Tumors, Paraganglioma of the Hypoglossal Nerve. A Case Report

\section{Edmond NUELLARI}

Author Information:

Angiosurgery Service, University Hospital Center "Mother Theresa", Tirana, ALBANIA

\footnotetext{
Abstract

Paraganglioma is a rare neuroendocrine tumor that originates in neural crest. There are a few instances mentioned of paraganglioma of the hypoglossal nerve even in worldwide scientific literatures. Paraganglioma of the hypoglossal nerve near the arteria carotis cannot be determined beforehand without a surgical operation. It is a tumor mass of the neck visually indistinguishable from other commoner body tumors, such as carotis or vagus. Full recovery without functional defects of any kind can be possible through surgery of nerve preservation. Although, in some examples the elimination of cranial nerve XII is a necessity. Our medical case has similar symptoms described in scientific literatures. Images taken with contrastive Angio Scanner and angiography of ACI, ACC and vena jugularis interna reveal a hyper vascular mass in carotid bifurcation. As in previous reported
}

cases, the source of paraganglioma was identified through histological and intraoperative methods. Nevertheless, our example is non-identical because the tumor was located near hypoglossal nerve. The tumor was later removed. The patient had no difficulties in speech articulation, only in swallowing. After 5 days of tumor removal, the condition beganimproving. After 3 months, the situation went back to normality.

Conclusions: Paraganglioma is a rare neuroendocrine tumor that originates in neural creste. It is a tumor mass of the neck visually indistinguishable from other commoner body tumors, such as carotis or vagus. Full recovery without functional defects of any kind can be possible through surgery of nerve preservation. Although, in some examples the elimination of cranial nerve XII is a necessity.

Keywords:Paraganglioma of hypoglossal nerve, swallowing, contrastive Angio Scanner.

$O P-188$

\section{Subclavian Artery Atherosclerotic Disease (SAAD)}

Marsela SOPIQOTI ${ }^{1}$, Edmond NUELLARI ${ }^{1}$, Frenki VILA ${ }^{3}$, Ervin BEJKO $^{2}$, Sokol XHEPA ${ }^{1}$

Authors Information:

${ }^{1}$ Service of Angiology \& Vascular Surgery UHC

"Mother Teresa" of Tirana, ALBANIA

${ }^{2}$ Service of Anesthesiology \& Intensive Care UHC

"Mother Teresa" of Tirana, ALBANIA

${ }^{3}$ Service of General Surgery University Hospital

Center "Mother Teresa" of Tirana, ALBANIA 
touch, and in severe cases, digital ischemia has also been described.

Case: We report a rare case of a 54-year-old man with subclavian atherosclerotic disease, presented with a 4-day history with mild pain and tenderness of the upper left extremity. His past surgical history was significant for an aorto-bifemoral bypass grafting 5 years ago for aortoiliac occlusive disease. After taking in consideration the location of the stenosis, the patients clinical state, physical parameters and the state of the occluded vessel surgical intervention was required, consisting in carotid - subclavian by-pass. Clinical and radiologic tests suggest atherosclerotic occlusive disease. We therefore discuss in our presentation the surgical management of the disease, along with the post operatory course of the patient.

Discussions: Endovascular repair with or without stenting has been proposed as a first line treatment by many authors, especially for patients of higher surgical risk. Concluding, Open and endovascular treatments of patients with atherosclerotic subclavian artery disease show similar early outcomes, although open repair was more frequent in their study in patients with subclavian artery occlusion. In the long term, open repair shows a significantly higher primary patency, without any difference in overall survival or symptom recurrence. Therefore, even in cases formerly addressed as high risk, open repair could be a safe and durable strategy, although hospital stay seems to be longer and randomized data are lacking. Novel endovascular techniques, such as the use of covered stents or drugeluting devices, may alter outcomes in the future.

Keywords: Atherosclerosis, subclavia artery, bypass.

$O P-189$

Multidistrectual Thromboembolic Events with Myocardial Infarction, and Acute Ischemia of both Legs in a young Woman Diagnosed with Antiphospholipid Syndrome

Denis KOSOVRASTI ${ }^{1}$, Frenki VILA ${ }^{3,}$

Daniela TEFERIÇI ${ }^{5}$, Petrika GJERGO ${ }^{1,}$ Ervin BEJKO $^{2}$, Sokol XHEPA ${ }^{4}$
Authors Information:

${ }^{1}$ Service of vascular surgery, University Hospital Center "Mother Theresa ", Tirana, ALBANIA

${ }^{2}$ anesthesiologist, Cardiovascular Surgery, Uhc

"Mother Theresa", Tirana, Albania

${ }^{3}$ resident Of General Surgery, University Hospital Center "Mother Theresa", Tirana, Albania

${ }^{4}$ service Of Vascular Surgery, University Hospital Center "Mother Theresa", Tirana, Albania

${ }^{5}$ department Of Cardiology University Hospital Center "Mother Theresa", Tirana, Albania

Abstract (APS) is an acquired autoimmune disorder that can be manifested clinically as a recurrent venous or arterial thrombosis.

Case: We present a 26-year-old woman, with a 7year history of Diabetes Mellitus (DM type 1), that was firstly hospitalized in the Endocrinology department as a destabilized DM. She had a history of 3 spontaneous abortions. During her stay in Endocrinology the patient complained of chest pain associated with changes in ECG (negative T waves at V1-V4), and elevation of troponin I.2D echo revealed an apical dyskinesia and left apical ventricle thrombus measured $3 \mathrm{~cm} 2$.She was diagnosed as an anterior NSTEMI and underwent a coronarography procedure that showed one vessel disease, thrombotic material in proximal LAD. Triple therapy was started (IV heparin, aspirin, clopidogrel...)

During her hospitalization in ICU the patient complained of pain and paresthesia in the right leg. An angio -CT of the chest, abdomen and the peripheral extremities revealed: lineal infarction, bilateral parenchymal renal infarction and thrombotic occlusions of the right and left popliteal arteries. Bilateral embolectomy with Fogarthy probe was performed permitting vascularization of both occluded vessels. Another angio-CT showed no evidence of thrombus in both popliteal arteries.

Discussion:The antiphospholipid syndrome is a rare disease. The clinical criteria for its diagnosis were: evidence of thrombosis and 
repeated fetal loss. Close collaboration with vascular surgeons should be always possible in order to perform revascularization interventions during the acute phase of the thrombotic event.

$O P-190$

\section{Laser Treatment in the Management of Chronic Venous Disease}

Skender VELIU ${ }^{1}$, Jasmina KRÖPFL ${ }^{2}$, Dominika VRBNJAK ${ }^{3}$

Authors Information:

${ }^{1}$ Surgeon, Surgical ward General Hospital $d r$. Jože Potrč Ptuj Potrčeva Ptuj, SLOVENIA

${ }^{2}$ Nurse for controlling hospital infectionGeneral Hospital dr. Jože Potrč Ptuj SLOVENIA

${ }^{3}$ Assistant Professor University of Maribor Faculty of Health Sciences Žitna Maribor, SLOVENIA

\begin{abstract}
Shortly after 1960, laser became an indispensable tool in the field of medicine. Due to continuous development of laser technology and optical systems, the use of laser in medicine has been a constantly advancing. In medicine, the laser is mainly used in the field of ophthalmology, surgery, dentistry and aesthetic skin treatments.In General hospital dr. Jože Potrč Ptuj laser is used in the field of surgery for surgical treatment of varicose veins and a supportive treatment of chronic wounds. The paper presents the basic characteristics of the minimally-invasive thermal ablation techniques - Endovenous laser ablation (EVLA), experiences and results of treatment using this method in General hospital dr. Jože Potrč since 2016 are presented. The basic characteristics of the $\mathrm{K}$ laser, experiences and the $\mathrm{K}$ laser outcomes of phototherapy treatment of patients with chronic wounds of different etiologies are presented as well. $\mathrm{K}$ laser is used as a supportive therapy in hospital since 2018.
\end{abstract}

Keywords:laser, endovenous laser ablation, phototherapy, K laser.

$$
O P-191
$$

\section{Ruptured Abdominal Aorta Aneurysms}

\author{
Petrika GJERGO ${ }^{1}$, Ervin BEJKO ${ }^{2}$, Stavri \\ LLAZO $^{2}$, Edmond NUELLARI ${ }^{1}$, Sokol \\ XHEPA $^{1}$ \\ ${ }^{1}$ Clinic of Vascular Surgery, UHC Mother \\ Theresa, Tirana, ALBANIA \\ ${ }^{2}$ Service of Anesthesiology and Reanimation, \\ UHC Mother Theresa, Tirana, ALBANIA
}

\section{Abstract \\ Background: Abdominal aortic aneurysm} (AAA) is a common and life threateningdisease that affects $5-9 \%$ of the population over the ageof 65 years. AAA is more common in male smokers with a positivefamily history of aortic aneurysms. Most patients with AAAare asymptomatic unless they develop a complication. Rupture, the most common complication of AAA, is one of the most fatalsurgical emergencies; it has an overall mortality rate of approximately $90 \%$. Most clinicians know that patients with ruptured AAA presentwith a classical triad of back pain with or without abdominalpain, hypotension, and a pulsatile abdominal mass.

The purpose of this study was to present our 3-year experience regarding the ruptured AAA.

22 patients with ruptured infrarenal abdominal aortic aneurysm presented in our clinic since january 2017, 19 males and 3 females, mean age 74.3 years. All patients had an acute presentation with abdominal and back pain and collapse. 7 patients presented within 6 hours from the beginning of symptoms, 9 patients presented within 12 hours and 6 patients after 12 hours. Patients were diagnosed using angio-CT scan, they all had a retroperitoneal hematoma. 15 patients underwent emergent surgical treatment of ruptured AAA, while for the other 7 patients, their relatives refused the surgical intervention. From the 15 treated patients, only 2 patients died, one because of an aortocaval fistula, discovered during surgery, and the other one due to cardiac arrest. 
In conclusion, patients with ruptured AAA should be treated immediately through surgical intervention, despite the operative risks. The early diagnosis enables the surgical treatment with an acceptable mortality.

$O P-192$

Inferior Limbs Amputations in Diabetic Patients Following Long-Term with Artherial Complications in Vlore's Regional Hospital.

\section{Petraq MUSTAQE ${ }^{1}$, Edmond BRESHANI ${ }^{2}$}

Authors Information:

${ }^{1}$ Surgeon-Angiologist At Regional Hospital of Vlora, ALBANIA

${ }^{2}$ Anesthesiologist At Regional Hospital Of Vlora, ALBANIA

\begin{abstract}
Introduction:In this article we are discussing the epidemic spreading of the "diabetic foot" in diabetic patients because of the peripheral vascular disease at many levels of the lower limbs (from femoral level to the foot). We have recorded 92 amputations in our Hospital from 2000 - 2015, in a city that counts 150,000 inhabitants.

In this study we have recorded 92 cases of lower limb amputations in both genders with 62 males and 30 females, varying from $50-80$ years old. 42 of these cases have gone through a femoral amputation of one limb. 12 cases have gone through crural amputation of one or both limbs. 18 cases of Lisfranc amputations and 16 cases of atypical amputation. In this study we have also included 4 cases of upper limbs amputations due to micro arterial complications in nondiabetic patients and patients with Raynaud syndrome.

In 4 of these cases after the crural amputation we have needed a reintervention that led to the amputation of the lower limb at a higher level. Also, in 8 cases with Lisfranc amputation we have needed a reintervention trying to save the calcaneous bone and in two cases we went on to amputate at crural level. In most of the cases we have performed a histological exam of tissues and vases. In all cases we have observed the formation of calcific deposits that have transformed the
\end{abstract}

walls of the vase in a rigid structure. The vases are occluded (from $85 \%$ of the lumen to $100 \%)$ in all of the cases. The muscular tissue below the amputation line were necrotic, denaturated, non-vital and we have been obliged a few times to go above the amputation line in order to find vital and conserved tissue.

Keywords:amputation, diabetic gangrene, atypical amputation, Lisfranc amputation, femoral amputation.

$O P-193$

Difficult Vaginal Delivery: The Need to Remodeling Postgraduate Program Focus in Obstetrics and Gynecology in Albania and not Only

Zef DELIA $^{1}$, Rustem CELAMI ${ }^{2}$

Author Information:

${ }^{1}$ Professor at University of UET, Obst Gynecologist, American Hospital of Tirana, ALBANIA

${ }^{2}$ Professor at University of Aldent, Obst Gynecologist, American Hospital of Tirana, ALBANIA

Abstract

Introduction:Vaginal delivery is a physiological and natural process of birth, when happens within normal parameters of definitions well recognized by respective national and international professional official organizations.

With the trend of increase rate of Cesarean section, worldwide, and in Albania together with lack of focus, and lack of mentorships in clinical skills of post graduate programs in obstetrician gynecologist specialty, we face and will face even more problems in managing normal and especially abnormal vaginal deliveries.

According to latest data, only shoulder dystocia accounts up to $1 \%$ (with median values of $0.5-0.7 \%$ ). The diagnosis of dysfunction of three main elements like dynamics, mechanic and plastic one should 
be done on time and with professional objective in order to minimize to risks to fetus and mother. Shoulder dystocia occurs when anterior fetal should, most commonly, and posterior fetal shoulder, less commonly, impacts on symphysis or sacral promontory. Morbidity and mortality associated with this dysfunction of normal delivery is significant.

This presentation purpose is to bring awareness of the fetal and maternal trauma that can occur on difficult delivery and to bring attention of remodeling academic postgraduate programs in order to more medical professionals of respective field to profit from clinical skill and offer e better medical care to this subgroup of patients

Keywords:Difficult, vaginal delivery, remodel, postgraduate, program

$O P-194$

This abstract has been withdrawn.

$O P-195$

Vaginal Cuff Dehiscence as Cuff Tear, Separation, Rupture; Our Experience

Avenir BALILI ${ }^{1}$, Rustem CELAMI ${ }^{1,2}$

Author Information:

${ }^{I}$ Obstetrician-Gynecologist, American

Hospital of TIRANA, ALBANIA

${ }^{2}$ Professor at University of Aldent, TIRANA, ALBANIA
Abstract
Introduction:Most common post hysterectomy complications can be categorized, such as; bleeding, infections thromboembolic event, urinary or intestinal injury, nerve lesions and lastly vaginal dehiscence.
In general, all surgical wounds in normal, healthy individuals heal through an orderly sequence of physiologic events which

include; inflammation, epithelialization, fibroplasia and maturation. Mechanical failure or failure of wound healing at the surgical site, may lead to disruption of wound closure and it is followed by seroma, hematoma, wound dehiscence or hernia.

Abdominal wound dehiscence is a rare postoperative complication of total hysterectomy, but vaginal cuff tear is very rare. Evisceration through vaginal opening, is life-threatening surgical emergency. In this presentation will be reviewed, the risk factors, symptoms, precipitating events and symptoms, and treatment.

It is imperative to maintain a high index of suspicion in patients who underwent laparoscopic or robotic hysterectomy more frequent nowadays.

Keywords:Hysterectomy, complication, vaginal cuff, dehiscence

$O P-196$

This abstract has been withdrawn.

$O P-197$

Deep Infiltrating Endometriosis - The
gynecologist Challenge

Leon KAZA

Author Information:

Obstetrician and Gynecologist, Lezha Regional Hospital, ALBANIA
Abstract
Introduction;Endometriosis is a chronic gynecological disorder that affects approximately $10-15 \%$ of women in reproductive years being the second most common benign disease in the female population. The heterogenic clinical picture of endometriosis with varies symptoms can seriously compromise the physical, psychological and quality of life of patients. Deep Infiltrating Endometriosis is defined as endometriosis penetrating more than $5 \mathrm{~mm}$ beneath the peritoneal surface. It is estimated that $10 \%$ of cases of endometriosis will have deeply infiltrating lesions. This form of the disease remains the most difficult to treat and 
needs highly skilled surgeon. Surgery must be performed by surgeons who are dedicated to endometriosis and treatment should be based on a collaborative multidisciplinary approach. In Albania deep infiltrating endometriosis remains still unknown, so much more must be done in order to diagnose this condition that affects seriously women quality of life. In our speech we will talk about some features of deep infiltrating endometriosis and especially on preoperative diagnosis of the disease.

Keywords:Endometriosis, gynecologic, disorder, infiltrating, diagnosis, treatment

$O P-198$

This abstract has been withdrawn.

OP - 199

This abstract has been withdrawn.

$O P-200$

This abstract has been withdrawn.

$O P-201$

This abstract has been withdrawn. 


\section{POSTER EXHIBITIONS PRESENTATIONS}

$P E \& P-1$

This abstract has been withdrawn.

$$
P E \& P-2
$$

This abstract has been withdrawn.

$$
P E \& P-3
$$

\section{Drowning and the Importance of CPR First Aid.}

Studim HYSA.

Author Information: University "Aleksander Xhuvani", Faculty of Medical Sciences, Elbasan ALBANIA.

\begin{abstract}
Introduction:Drowning is the experience of being unable to breathe as a result of strained and uncontrolled immersion in water.According to the WHO (World Health Organization), 500,000 deaths per year or $0.7 \%$ of deaths are caused by accidental drowning.Brainenergyconsumption drops by $5 \%$ for every 1 degree below 37 degrees, in the range of 37 to 20 degrees.After identifying and taking the person out of the danger zone: laying a person on a strong plinth. We place the closest First Aid box on our arm to look at the person if he or she responds to painful speech, congestion or nflammatorystimulants. We release the Breathing Paths from the garment and do the head extension with 2 fingers on the chin and hands on the forehead by raising the head which allows the air to circulate freely.We examine the mouth cavity for foreign bodies, we remove the dentures and fluids and cleanse the mouth. controlling Inspiration: Listening, observing, feeling. The first helper holds his/her cheek and ear close to the person's mouth and eyes toward his chest to see his movements.
\end{abstract}

Keywords: Drowning, breathe, examination, resuscitation.
$P E \& P-4$

\section{Innovation in Radiology}

Najada KALLASHI (JAHIQI) ${ }^{1}$, Erjona ZOGAJ $^{2}$

Authors Information:

${ }^{1}$ Radiologist, University Trauma Hospital, Tirana, ALBANIA.

${ }^{2}$ Imaging technique, University Trauma Hospital, Tirana, ALBANIA.

\begin{abstract}
Innovations in radiology have led to essential and irreplaceable developments in the field of medicine by improving the quality of patient care and reducing mortality rates. Various imaging modalities have brought about revolution in the field of medicine over the years. Hybrid imaging is one of the latest innovations being widely used and bringing major developments in radiology.

Radiology is a medical field that works the practice of imaging to both analyze and treat disease imagined within the body. Radiologists use a collection of imaging technologies such as $\mathrm{X}$-ray radiography, ultrasound, computed tomography (CT), nuclear medicine, positron emission tomography (PET) and magnetic resonance imaging (MRI) to analyze or treat diseases. Interventional radiology is the usually minimally invasive medical procedures with the guidance of imaging technologies. Innovations are new idea, device or process. Innovations are the application of better solutions that meet new requirements, in articulated needs or existing market needs. It is proficient through more effective products, processes, services, technologies, or new ideas that are readily available to markets, governments and society. Innovations are something original and novel, as a significant, new that breaks into the market or society.
\end{abstract} Keywords:Radiology, medicine disipline, innovation, hybrid imaging.

$P E \& P-5$

Emergency Trauma Management, Epidemiology of Trauma

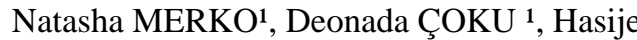
DURAKU ${ }^{1}$, Etleva RAMALIU ${ }^{1}$ 
Authors Information:

${ }^{1}$ Nurse Service University Hospital of Trauma, Tirana, ALBANIA.

\begin{abstract}
Introduction: In our Emergency Department, at University Hospital of Trauma, everyday come approximately 50-70 patients whom we offer our service. Many of them are from different accidents and came to the hospital immediately, and others from regional hospitals. On average 4-5 patients are polytraumatized patients. Management of patients at Emergency Department has many challenges. Being the only Trauma Center, it deserves a special attention for all the problems that may have the Emergency Department.
\end{abstract}

Materials and Methods; A sectional study was performed on 200 patients in trauma emergency for a period of 2 months from 1.11.2013 to 31.12.2014.

Results : Males are the main victimes, and the road accident are the main causes. Damages more than 2 specialties, coordination between specialties for diffèrent choices in managing patients in their delivery. Conclusions: The key of success for a good management is a special trauma team and standart protocols. Only this way we can offer a great service, and have a decreased mortality and morbidity.

Keywords; Politrauma,selection, trauma center, emergency department.

$P E \& P-6$

DMFT (Decayed Missing Filled Teeth) Index and Socioeconomic Status in Adult Patients

Ramona A. PARISI

Author Information:

Dentist at Dentale Clinic "Parisi" Tirana, ALBANIA

\footnotetext{
Abstract

Introduction: This study was conducted to evaluate the association between DMFT Index and socioeconomic and behavior al risk factors in randomly selected adult patients.
}

Materials and Methods: Socioeconomic data for each patient was extracted by a questionnaire(short generic set of questions on health-related quality of life, educational level and achievement, occupation) and medical cartels of patients. (Dental Clinic "Parisi")

DMFT Index was categorized as: DMFT $>10$ and DMFT $<10$

Results: The study concluded that the prevalence of DMFT was found more (DMFT $>10$ ) in lower socioeconomic status when compared to uppers socioeconomic status $(\mathrm{DMFT}<10)$

Keywords: DMFT, Socioeconomic Status, Adult Patients.

$P E \& P-7$

First Aid in Management of Trauma Patient.

Natasha MERKO ${ }^{1}$, Albana NDOJ ${ }^{1}$

Authors Information:

${ }^{1}$ Nurse Service University Hospital of

Trauma, Tirana, ALBANIA.

\section{Abstract}

Introduction: September 12, World Aid

Day. Many people are unable to approach an emergency service, act correctly and consequently have no loss of life, or deterioration of health. This requires more information and concrete action across the community in order to enable them to deliver. first aid according to certain rules, seeking more specialized services in order to avoid as much as possible loss of life and complicating situations Organizing this day by all health structures in cooperation with other structures, including activities, publications, direct community outreach, practical demonstrations in public, or the most diverse forms that can be accomplished for this purpose is a good opportunity to educate the public on correct behavior and knowledge.

Materials and Methods:A sectional study was done on 190 patients in Tirana 
Emergency on the scene for a 2-month first time service, from 1.11.2014 to 31.12.2014.

Results:Out of 210 patients it turns out that men include the main victims of road accidents, $85 \%$ men $25 \%$ who are female patients. The age of these patients ranged from 2-85 years for males, and from 3-70 years for females, with an average of 32 years old. Most patients with trauma Official and age groups of 15-30 years of age from 31 to 45 years old. It is stated that patients come from road: Lushnje-Fushe-Kruja, TiranaDurres, Kombi road.

Conclusions: A well-trained team specializing in trauma management with a standard procedure and protocols, well defined are the keys to effective management of polytrauma patients.

Keywords: emergency, emergency, ventilation, rating, transfer, selection.

$P E \& P-8$

\section{Gestational Diabetes}

Natasha MERKO ${ }^{1}$, Yllka BARDHI ${ }^{1}$, Etleva RAMALIU 1

Authors Information:

${ }^{1}$ Nurse Service University Hospital of Trauma, Tirana, ALBANIA.

\footnotetext{
Abstract

Introduction: Gravidance is a special condition of mother to become wwhere her body is preparing having a child. Pregnancy duration is based on the gestational age of the first day of menstruation visit, accepting a 28day cycle A fetus in term listed in the term he was born alive at 38-40 weeks, preterm before 37 weeks and a fetal serotonin or after the term is designated that fetus born alive after week 42 of pregnancy. and me gynecological and imaging examinations, diagnostics. Gravidancies more normal, likely or high performance, this classification can be based on complications, disorders or diseases may occur at the time of its diagnosis, duration and until its completion that is
}

birth.Complications increase the morbidity and mortality of neonat and mother too. The complication and the disease to be treated is a metabolic disease such as Gestational Diabetes or Pregnancy Diabetes.

Materials and Methods:.This study is a retrospective study, evaluating European and World literature, as well as Albanian. We are based on two Maternities in Tirana. "SUGJ Koco Gliozheni" and "Queen Geraldine" on cases of patients diagnosed with Gestational Diabetes.

Conclusions: Gestational diabetes is a complication, a very dangerous metabolic disorder in pregnancy, it risks two lives, both maternal and Fetal, increases the risk for neonatal morbidity and mortality. This study demonstrates that a special care by the multidisciplinary team in preventing it with an early diagnosis, and good gravid care at its infancy, and a more effective treatment of this pathology, eliminates the risk of mother and infant too.

Keywords: Gravidance, diabetes, glukosis, complications, prenatal diagnosis.

\section{$P E \& P-9$}

Multiseptated Gallbladder with Recurrent Abdominal Pain. A Case Report and Literature Review. Xhesika XHETANI $^{2 *}$ Vidi DEMKO ${ }^{1}$

Authors Information:

${ }^{1}$ Radiology Department, University Hospital of Trauma, Tirana, ALBANIA.

${ }^{2}$ Radiology Department, University Hospital Center "Mother Teresa", Tirana, ALBANIA.

\begin{abstract}
Introduction:Multiseptated gallbladder is a rare congenital malformation of the gallbladder and usually admits to the emergency services with differential symptoms such as right upper abdominal pain, nause and vomiting, and abdominal complaints.
\end{abstract}


We present a case of MSG with recurrent abdominal pain in which laparoscopic cholecystectomy resolves the complaints of the patient. Chronic abdominal pain is a common disorder in children and adolescents worldwide. In attacking this problem, the pediatrician often concentrates on the gastrointestinal or genitourinary tract as a source of the pain. Too little attention is paid to the gallbladder as the cause of pain in this age group. We report a 6-year-old girl who presented with recurrent abdominal pain and was diagnosed as having a multiseptated gallbladder, although rare, should be considered in the differential diagnosis of patients presenting with recurrent abdominal pain and abdominal ultrasonography should form part of the investigation.

In Conclusion;multiseptated gallbladder is a rare congenital anomaly and a rare cause of recurrent abdominal pain. Cholecystectomy is the choice of treatment in symptomatic patients such in our case. Our patients' abdominal pain and gastrointestinal complaints have resolved after surgery. Cholecystectomy should also be considered in elderly, asymptomatic patients in whom multiseptated gallbladder is incidentally discovered, due to the possibility of undetected carcinoma of the gallbladder Keywords:gallbladder, abdominal pain, ultrasonography.

$P E \& P-10$

\section{Role of Emergency Department in University Hospital of Trauma}

Edlira LTC KUKELI ${ }^{1}$, Raimonda TRAKO ${ }^{2}$

Authors Information:

${ }^{1}$ military Medical Unit, Trauma University Hospital, Tirana, Albania

${ }^{2}$ american Hospital, Tirana, Albania

\section{Abstract}

Introduction:The emergency department is staffed and equiped to provide rapid emergency care for acute illness, severely and multiple traumatized patients. Trauma is a major cause of death in recent years The emergency departament use the triage system, determinate the priority of needs (available personnel, equipment), stabilize and transfer the patient under specialised care of trauma teams. The goal of emergency care is to reduce the mortality and morbidity by preventing of complications. Actually, the trauma care is the priority of clinical research in developing countries.

Material and Method:The retrospective study was performed during the period of time (2014 -2018). It has thrown interesting statistical data which are collected in medical records of Trauma University Hospital.

Results: Data collection related with admitted patients in emergency departament. There is increasing of (trauma+emergencies) incidence from 2014 until 2018. The incidence resulted 62,8\% (2014), 73,5\% (2015), 70,1\% (2016), 99,4\% (2017), 97,4\% (2018).

Conclusion:The emergency department is the part of medical polytrauma support.The training courses are neccessary and avaiable for trauma medical teams. Improving of he cooperation and coordination between facilities is the best way for effective emergency care.

\section{$P E \& P-11$}

\section{Hepato Cellular Carcinoma. A case Report}

Etmont ÇELIKU, Manser ÇILI*.

Authors Information:

Department of Surgery, University Hospital Center "Mother Theresa" Tirana, ALBANIA

Abstract
Hepatoma is a tumoral disease that consists in
an abnormal proliferation of the hepatic cells.
According to the augmentation of the risk
factors such as smoking, alcoohol abuse,
consuming no healthy food and different
types of viral and bacterial infections etc.
Nowdays, this disease is becoming more 
evident even in the young ages. Here, we present a 63 old female with this disease

Hepatoma is malignant disease that is also known as hepatocarcinoma (HCC) and is one of the most expressed tumoral disease of liver. It is seen a strong correlation between hepatocarcinoma and other chronic liver disease such as Hepatitis B, C, diabetes mellitus type 2, or chirrosis etc.

Also is evidenced that being exposed to harmful factors such as alcohol abuse or being malnutried and with hipercaloric diet mainly in cholesterol and triglicerides (that cause the fat's accumulation in liver) can bring an augmentation of Hepatocellular carcinoma disease. However, this disease is very similar in appearance with the metastasis that origins from the other organs such as colon, stomach etc.

Most cases are treated with surgical resection and chemotherapy, but the prognosis is not very hopeful.

The hepato-carcinoma disease is very malignant disease that interests not only the liver, but all the physiological reactions processes and has a high risk to spread metastasis in the other organs. Being in front of this situation in this case, we thought that the most suitable way to proceed is the surgical treatment followed by the chemotherapy.

The patient today is alive and has an improved clinical health.

\section{$P E \& P-12$}

\section{Some Considerations about Pancreatic Cancer in Albania}

Aurela KULLOLLI ${ }^{1}$,Xheladin DRAÇINI ${ }^{1,2^{*}}$, Etmont ÇELIKU ${ }^{1,2}$

Author Information:

${ }^{1}$ General Surgery Services, UHC "Mother Theresa" Tirana, ALBANIA

${ }^{2}$ University of Medicine of Tirana, Faculty of Medicine, Tirana, ALBANIA

\footnotetext{
Abstract

Introduction: Pancreatic Cancer is the seventh leading cause of cancer-related deaths worldwide and the third most common in the USA. There are few studies in Albania. The aim of our study is to evaluate epidemiology, history, diagnostic procedures and surgical treatment of pancreatic cancer in Albania.
}

Material and Methods: This is a retrospective study. In this study we reviewed all patients diagnosed with pancreatic cancer in Service of Surgery No.1, Surgery Department at University Hospital Centre "Mother Theresa", Tirana, Albania in the period between January 1-st 2017 and March 31-st 2019. All data were collected from medical records.

Results: Our study shows that pancreatic cancer is a low-incidence pathology that affects men more. The most affected age group is 61-70 years old with an average age of 66 years. Our study included 66 patients, of whom 28 belonged to 2017, 28 to 2019, and 10 cases to the first 3 months of 2019 . The study found that pancreatic cancer was diagnosed at a later stage and the most common surgery was $84 \%$, palliative surgery, while $8 \%$ receive conservative treatment.

Conclusion: Challenges to face are early diagnosis and treatment of Pancreatic Cancer.

Keywords: Pancreatic Cancer, Pancreatic Ductal Adenocarcinoma,

$P E \& P-13$

\section{Life Quality of Immobilized Patients}

\section{Zamira SHABANI ${ }^{1}$, Edona HAXHIJA ${ }^{1}$, Donika SHKOZA $^{1}$}

Authors Information:

\author{
${ }^{1}$ University of Shkodra "Luigj Gurakuqi" Faculty \\ of Natural Sciences, Department of Nursing, \\ Shkoder ALBANIA
}

\begin{abstract}
Introduction: This is a transversal (crosssectional) descriptive study. The aim of this study is to evidence of quality of life in immobilized patients.

To identify physical skills related to daily activities in immobilized patients. Identify the level of dependence of immobilized patients and need for help. Identify the quality of life of immobilized patients regarding age group and diagnosis.
\end{abstract}

Materials and Methods:The data collection was carried out in the period from January 
2019 to May 2019. The sample was selected randomly. In this study were included 85 immobilized patients. Initially, we tried to include 100 patients but 15 of them refused to cooperate. Immobilized patients were provided with a standardized international questionnaire administered by us. The questionnaire used contains 10 questions through which we obtain the necessary information regarding the immobilized patient's ability to perform basic need. This questionnaire is called the Bartel Index originally created by Florence I. Mahoney and Dorothea W. Barthel; in use since 1955, first published in 1958). Questionnaires with patients were conducted at QSUT "Mother Theresa". Before the interview began, patient and family consensus were taken. To analyzed the statistical data was used the SPSS.Vers.19 and Microsoft Office 2010. Was applied the Pearson correlation for IC $=99 \%, \mathrm{p}<0.01$.

Results:The average age of the interviewed patients is 62.5 years old when the minimum age is 22 years old and the maximum age is 96 years. $53 \%$ of these patients are male $(\mathrm{n}=$ $45)$ and $47 \%$ are female $(n=40)$.

Conclusions: Most patients are unable to perform such actions as personal grooming personal effects, making the shower itself, climbing / lowering the stairs. 1/5 of immobilized patients have complete dependence on their daily activities and present us with a very bad life quality. Almost half of the immobilized patients have severe dependence and are presented with a poor quality of life. Heavy dependence is closely related to age increases. The higher the age of patients the higher the dependence and the lower their quality of life associated with daily activities.

Keywords:Ability, immobilization, patient, dependence.

\section{$P E \& P-14$}

\section{Gouty Artheritis, Correlation with Other Metabolic Disease}

\section{Alban HYSENAJ}

Author Information:

Orthopedic Resident, University Hospital Center "Mother Theresa", Tirana, ALBANIA

\begin{abstract}
The association of the gut with other associated pathologies that make up the metabolic syndrome is well known. The gut has been seen to be associated with obesity. Numerous studies have indicated that a large percentage of these patients have body weight above ideal relative to age and body length. Hyperuricemia is common in diabetic patients, whereas glucose arthritis is rarely encountered. Impaired glucose tolerance tests have been reported in $7-74 \%$ of patients with gut, depending on the criteria used.

Hypertriglyceridemia has been reported in $75-80 \%$ of patients with gut, and hyperuricemia was found in more than $80 \%$ of patients with hypertriglyceridemia. Numerous studies have failed to show a correlation between urate and cholesterol levels. Hypertension has been found in $1 / 4$ to $1 / 2$ in patients with gut, but the presence of hypertension is unaccompanied by prolongation of the gut. Many studies have seen an association of hyperuricemia with atherosclerosis manifestations and hyperuricemia has been proposed as a risk factor for coronary disease. Alcohol use is associated with hyperuricemia and predisposing persons may have. Based on these facts, we undertook to study with our modest contribution the association of these conditions with arthritis.
\end{abstract}

Keywords:Gut, hyperuricemia, hypertension, associations with arthritis.

$P E \& P-15$

\section{Rehabilitation of Polytrauma Patients and Tertiary Damage Reduction}

Manushaqe SARAÇI ${ }^{1}$, Adriatik CEKA ${ }^{1}$, Geldon SPIROLLARI $^{1}$

Authors Information:

${ }^{1}$ Rehabilitation and Physiotherapy Service, University Hospital of Trauma, Tirana, ALBANIA.

\begin{abstract}
Introduction: The attitude of polytraumatic patients in Intensive Care extended because of the clinical conditions and consequently the occurrence of several aggravation factors, by preventing their transfer to rehabilitation facilities. In most cases patients that have respiratory problems, feeding etc.do not make
\end{abstract}


possible the application of a good rehabilitation program and to leave TI as soon as possible.

Material and Methods: This is a prospective study, involving 120 patients who were assisted in TI on the second day during December 2017- 2018.Average age 35+ 5years old. M/F 3:1(95M; 25F). Patients are presenting the evaluated cranial trauma with GCS 3-8 points. Extremity trauma: long bone and pelvic fractures, patients with thoracicabdominal and bone fracture polyinterventional and vertebral column trauma such as: paraplegia, tetraplegia. The rehabilitation protocol was applied in 3 phases: Phase I-Rehabilitation of patients in coma (Prevention of complication). Phase IIStart of motor commands. Phase IIIAutonomy (Post coma).

Results: Automobilist trauma was ranked first at $69 \%$. lower limb fractures and polyintervention surgical: The duration of rehabilitation procedures is 4-6 weeks. Up to $80 \%$ positive results. Cranial traumas: 6 patients with depase-coma died during the first week, 10 patient's irreversible coma. Rehabilitation period is 6 months to 1 year.60-65\% positive results. As a result invalidity is high.

Conclusion: Rehabilitation starting on the second day to patients with polytrauma in ICU we minimized tertiary damages as decubitus, paraarticulare calcification, muscular hypotrophy, urinary and pulmonary infections, etc, so we reduced disability.

$P E \& P-16$

This abstract has been withdrawn.

$P E \& P-17$

Trauma Emergency Surgery and Management of Infectious Disease

Petrit BIBERAJ, Blerina BAHITI, Florenc PILIGRIU

Authors Information:

Faculty of Medical Technical Sciences, University of"Aleksander Xhuvani" Elbasan, ALBANIA.

\section{Abstract}

Accidents and trauma are one of the world's mostserious but neglected health problem.
The fast-movingtransportation systems, unprecedented and unplannedurbanization and changing social patterns have contributedto the global increase in the incidence of trauma to human

body. Traffic accidents are an endemic disease which affectsmainly the young adults in the economically productive agegroups and are the leading cause of death in persons under44 years of age. Globally, 26\% of all deaths in the agegroup of 15-44 years in 2015 were due to injuries.

Infections in Traumatized Patientsare second only to head injury as the leadingcause of death beyond the first three to four days of traumaand are responsible for $80 \%$ of late deaths in adult traumapatients. This is mainly because the management ofother major contributors of mortality like hemorrhage,circulatory collapse and respiratory failure has greatlyimproved with the application of sophisticated technicaladvancements. Traumatized patients with infectionshave a 5-fold higher mortality compared to those withoutinfection. The overall incidence of infections followingtrauma varies from 9-36\%.This is much higher thanthe rates of nosocomial infection for general population. The overall mortality was $15 \%$, with majorityof death due to infections.

The financial implication ofinfection and sepsis following trauma are also staggering. Resource utilization is prolonged in traumatized patientswith infections, most of which is spent in the ICUs. Thecost of caring for these patients will continue to increase with inflation, newer techniques, more sophisticatedmonitoring and the advent of new therapies.

$P E \& P-18$

Role of MRI in Cervical Spondylosis Myelopathy with Other Pathological Findings

Bardha DERVISHI ${ }^{1}$, Jeton SHATRI, Agon TURKAJ ${ }^{2}$, Veton ADEMI

Author Information:

${ }^{1}$ Student of General Medicine, University Clinical Center of KOSOVO

${ }^{2}$ University Clinical Center of KOSOVO 


\begin{abstract}
Introduction: Cervical spondylotic myelopathy (CSM) is a neck condition that arises when the spinal cord becomes compressed due to the wear-and-tear changes that occur in the spine as we age.
\end{abstract}

Case Report: The patient was a 52-year-old male, who complains of neck pain, paranesthesia in upper extremities, lower back pain and bilateral calf pain, muscle weakness in the lower and upper extremities, fatigue and general body pain that started four weeks ago associated with other clinical manifestation. Magnetic Resonance Imaging (MRI) of the cervical spine revealed canal stenosis and increased T2 signal within the spinal cord. CSM is a rare condition there are very few or no cases described in literature when CSM in C3/C4 is associated with a lack of B12 and other pathologies such as and hypoplasia of the mastoid air-cell with system maxillary sinus aplasia.

Conclusion:The case described in the present study suggested that the incidence of CSM may be correlated with VB12 deficiency, particularly for cases in which the clinical manifestations and the imaging do not fully match.

Keywords:Cervical myelopathy, clinical characteristics, hypoplasia of the mastoid aircell

system, MRI, prognostic value.

$P E \& P-19$

This abstract has been withdrawn.

$P E \& P-20$

Patient Satisfaction and Quality of Service: Trauma Hospital Case

Rezarta KALAJA ${ }^{1}$

Authors Information:

${ }^{1}$ Head of Department of Medical Technical Sciences; University "Aleksandër Moisiu” Durrës ALBANIA

\section{Abstract}

Introduction:Patient satisfaction is an important indicator for measuring the quality in health care. The purpose of previous studies in this field has always been to assess whether there is an impact on the evaluation of patient satisfaction in improving service quality. Already most of the healthcare industries are more and more concentrated on patient centered care and patient satisfaction, so measuring and analyzing patient satisfaction is an indispensable tool for enhancing and improving service quality. Health care organizations have been talking about and focusing on patient experience and patient satisfaction for a long time. Health systems should use patient satisfaction as a balance measure not a driver for outcomes (Bickmore and Merkley, 2019).

This paper is based on a questionnaire conducted at the Trauma Hospital, where 180 patients who had been hospitalized for at least two nights were interviewed. The method used is SERVQUAL, divided into demographic data and surveys related to the main determinants of service quality assessment. Kaneet et al. (1997) and Marley stated that measuring satisfaction should incorporate dimensions of technical, interpersonal and moral aspect of care. In this study the determinants used to evaluate quality by patients were, communication with clinicians, responsiveness of hospital staff, cleanliness and quietness of the hospital environment, pain management, empathy of staff and use of advance technology. The purpose was to find out whether patients' perceptions of service quality were related to their satisfaction, or whether the final outcome of treatment mattered to them alone. As a result, we can say that the satisfaction of patients was not only affected by the outcome of the treatment but also positively related to the way staff communicated, creating a sense of security and cleanliness and staff responsibility.

In evaluating hospital quality and enhancing patient satisfaction, an important element is not only the latter's evaluation, but also the communication of the results received to the staff, in order to take appropriate measures to improve the quality based on experiences and opinions of hospitalized patients. 


\section{INDEX TABLE}

$\boldsymbol{A}$

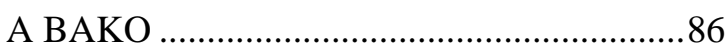

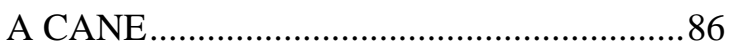

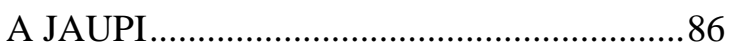

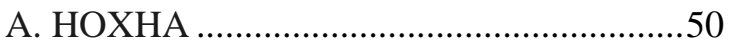

A. MASKA …………...................................93

Adelina XHEMALI .........................................30

Adem ZALIHIĆ..............................................44

Adham DARWEESH........................44, 46, 47

Admir MUSTAFA ................................46, 61

Admir NAKE .................................................30

Adriatik CEKA .............................................136

Adriatik DAKU .............................................48

Adriatik LAJÇI ...............................................90

Afërdita ADEMI ...........................................79

Afërdita KULLURI........................................74

Afrim JAHAJ …...............................................29

Afrim TAHIRI …..........................................14

Afron MICI ....................................................61

Agon TURKAJ ...................................57, 137

Agron DOGJANI .........................9, 27, 62, 63

Aida GAVRANOVIĆ ...................................44

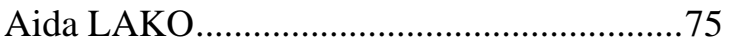

Aishe R LALA ...............................................35

Akgün ÇELIK .....................................................60

Alan ANDONOVSKI ..................................53

Alban HYSENAJ ..................................115, 135

Alban HYSENI ..............................................113

Albana ALEKSI................................87, 89, 90

Albana KENGA .............................................117

Amarildo BLLOSHMI....................................9

Albana KOÇIAJ.............................................27

Albana NDOJ .................................................131

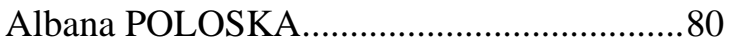

Albana SHAHINI.............................................48

Albert LLESHI..................................................70

Albert PESHA...............................................99

Albi ARKAXHIU ............................................58

Albiona SALIAJ ...........................................89

Aldi SHANI .....................................................91

Aleksandar SAVESKI ...............38, 54, 94, 113

Aleksandar TRAJANOVSKI...........38, 54, 113

Alfred AGA ….................................................41

Alfred GEGA ..............................................113

Alfred IBRAHIMI ............................10, 84, 85

Ali DEVAJA .................................................12

Alma CANI ........................................32, 35, 83

Alma LLUKAÇAJ ............................................51

Altin KUQO..................................................
Amire DRAGUSHA ........................................15

Ana STOJKOVSKA ......................................70

Andzela VITANOVA.......................................70

Aner DURAKOVIĆ ....................................... 44

Angela TRPOSKA......................................... 70

Anila RACAJ....................................55, 94, 97

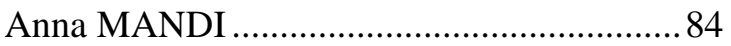

Antigona KABASHI......................................57

Antonio GAVRILOVSKI.................38, 54, 113

Antonio La GRECA ......................................... 8

Anxhela AHMATAJ.....................................73

Arb F. HOXHA ….........................................12

Arba CECIA …..................................19, 21, 23

Arben BEQIRI .............................................108

Arben DHIMA ............................................ 48

Arben GJATA.................................................. 10

Arben GJONEJ .............................................. 79

Arben LLOJA ….............................................. 102

Arben TUSHA ................................................... 81

Arbëresha GURGURI................................128

Ardiana SINANI...........................................66

Arjan MULLAHI............................................ 36

Arjana STRAKOSHA ….....................107, 108

Arjeta DEDEJ …..................................107, 108

Armand STAFUKA .....................................113

Armida KABA ................................................. 41

Armir DAMZI …….......................................50

Arnel MICI .................................................116

Arsel DIZDARI ...........................................113

Arsen SEFERI ….............................19, 21, 23

Arsim AJETI................................................ 12

Artan BODEC................................................... 50

Artan GJIKA...............................................46

Artan KONI ..................................................... 99

Artan OSMENLLARI ..................................66

Artid DUNI..................................................113

Artid LAME..................................................22

Artur XHUMARI...........................................220

Arvin DIBRA..................................10, 25, 50

Arvit LLAZANI...................................25, 50, 93

Asead ABDYLI .....................................69, 71

Asfloral HAXHIU ...........................................92

Astrit PEKA..................................................... 79

Astrit XHEMALI.............................50, 93, 118

Aulona HAXHIREXHA.......94, 103, 105, 107

Aurela KULLOLLI......................................134

Aurora KRASNIQI .......................................... 45

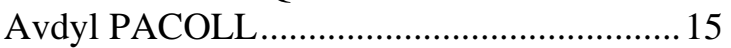

Avenir BALILI ..........................................124

Ayman EL-MENYAR ................................... 76 
B

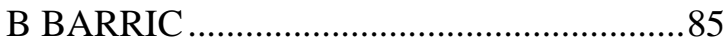

Bardha DERVISHI ...................................137

Bardhyl VEIZI .............................................46

Baris SAYGILI ...................................20, 22

Basri LENJANI...............................10, 28, 109

Behar TOCILLA ..............................................48

Besim BOCI.................................................40

Besim SERMAXHAJ ...................................14

Bijana POPOSKA ...........................................70

BiljanaANDONOVSKA...............................53

Bledar KRUJA …..........................................41

Bledar SHEGA.............................................99

Bledi CEKREZI .............................................48

Bledi MASATI...............................................92

Bleona SEJDINI...........................................75

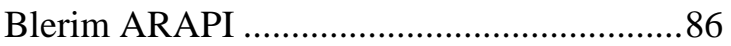

Blerina BAHITI ..........................................137

Blerta HAMOLLI ................................115, 116

Blerta HASALLA .....................................71, 72

Borys BILYK.................................................12

Bruna SUBASHI........................................128

Bujar GASHI ..................................................15

Burak KOZA...............................................111

Burim ELEZI ............................................110

C

Carlos MESQUITA ......................7, 24, 49, 51

D

Dalip JAHJA ..................................................38

Daniela TEFERIÇI........................................120

Daniela XHEMALAJ................................35, 66

Danny DAPHNIS................................124, 125

Dardan LENJANI .......................................28

Dariel THERESKA.......................................32

Denis KOSOVRASTI ..........................118, 120

Deonada ÇOKU ....................................82, 130

Deonada COKU (HYSKO).............................82

Desli SHAHINI.......................................58, 59

Dhimiter ARGJIRI.......................................32

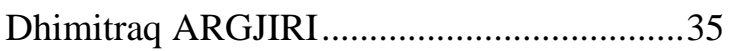

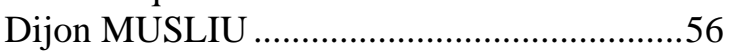

Dmitry ILYIN ................................................99

Dominika VRBNJAK ...........................13, 121

Donika SHKOZA..........................................134

Dorela VASHA ...............................................66

Dorian META …................................................... 73

Dorina SHQALSHI.......................................49

Dorina SHTJEFNI ...........................26, 35, 61

Drini SHEHI ...............................................25

Dritan TODHE .............................................115
$\boldsymbol{E}$

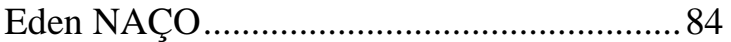

Edlira LTC KUKELI .................................... 133

Edmond BRESHANI..................................122

Edmond NUELLARI...........................119, 120

Edmond PISTULLI ........................................ 30

Edmond ZAIMI ..................................22, 28, 30

Edona HAXHIJA ........................................... 134

Edvin SELMANI ........................ 10, 54, 55, 79

Eliana SHIMA ….......................................... 83

Elizana PETRELA ..........................................2 27

Eljona XHELILI ............................ 91, 92, 118

Elona DYBELI ....................................71, 72

Elona GJYLBEGAJ............................. 106,126

Elona HASALLA …............................... 71, 72

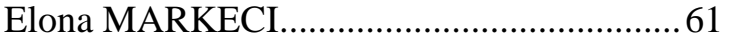

Elvis VELIU .................................................... 41

Emir SMAJIĆ ….............................................. 44

Emirjona VAJUSHI...................................... 41

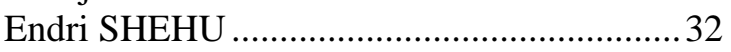

Endrit MONE ................................................ 75

Enea SERANI............................................... 30

Engin GÖÇMEN .............................................. 60

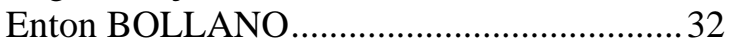

Enver FEKAJ ................................................. 14

Erald XHELILI.............................................. 18

Ergys CAMI....................................................113

Erinda KOSTURI …....................................... 45

Eriol BRAHOLLI ........................................... 92

Erion DREDHA .............................................. 100

ErionSPAHO ….............................................. 22

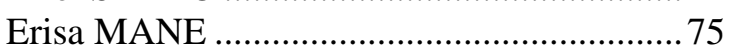

Eriselda TAULLA …………………...............64

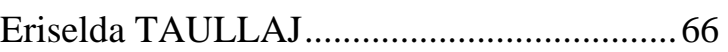

Erjona ZOGAJ ......................................10, 130

Ermira PAJAJ .....................................19, 21, 23

Ermira TOTRAKU .......................................67

Ervin BEJKO .................. 84, 85, 117, 118, 120

Ervin JONUZI …........................................ 95

Erza VOCA ..................................................... 28

Esat BARDHOSHI .......................................42

Esen UYSAL …...........................................28

Esma SHARKA …..................................64, 65

Esmeralda BULKU........................................ 84

Etleva GJOSHE ..........................................127

Etleva GJURASHAJ.......................................43

Etleva RAMALIU .........................82, 130, 131

Etleva SMAKAJ .............................................. 80

Etlleva RAMALIU ........................................... 81

Etmont CELIKU ............................................92

Etmont ÇELIKU ................25, 51, 84, 133, 134 
$\boldsymbol{F}$

Fadil GRADICA $10,32,35$

Fahri KOKICI .32, 35

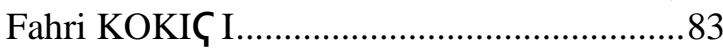

Fatbardha MYSLIMAJ ...................................67

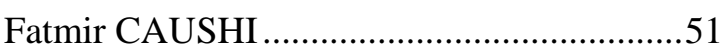

Fatmir ÇAUSHI ...............................................33

Fatmir GUNI .................................................43

Faton KRASNIQI ...........................................30

Faton T. HOXHA...........................................12

Ferdinand KOMANI .....................................12

Ferizat DIKA - HAXHIREXHA ....91, 94, 107

Ferizate HAXHIREXHA-DIKA..................103

Filip NAUMOSKI .......................................70

Fiona NASTO …....................................107, 108

Fisnik KURSHUMLIU ................................12

Fjorda TUKA ................................................48

Flamur VELLKU ...........................................38

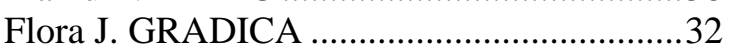

Flora ZYBERAJ...........................................80

Floren KAVAJA …...........................................12

Florenc PILIGRIU ........................................137

Florian DASHI.................................19, 21, 23

Frenki VILA...................91, 92, 117, 118, 120

$\boldsymbol{G}$

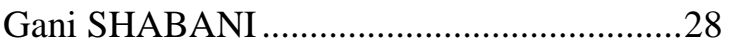

Gazmend ELEZI ...........................................110

Geldon SPIROLLARI................................136

Genc KABILI..............................124, 125, 126

Gentian CACO...............................................117

Gentian HUTI .......................................69, 71

Gentian ZIKAJ .......................................87, 88

Gentjana HASKJA ......................................128

Gezim BIBA …............................................113

Gëzim GURI ...............................................46

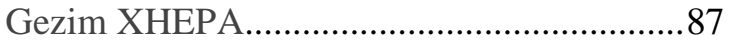

Gëzim XHEPA, ...............................................88

GezimGALIQI …........................................99

Gjergj CAUSHI ….........................................113

Gjergj ÇAUSHI .................................................36

Gjergj SEMINI..............................................125

Gjergji BELBA .......................................87, 90

Gjergji CAUSHI ........................................54, 55

Gjergji SEMINI .........................100, 101, 123

Gjovalin BUSHI.......................................35, 61

Gledion FEJZO …............................................22

Gramoz BRACE ...............................19, 21, 23

Guido BASSI .............................................113

$\boldsymbol{H}$

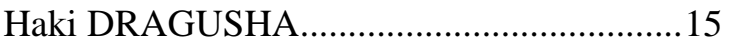

Haqif GASHI ...............................................16
Hasan HAFIZI .................................................... 66

Hasan Vedat OFLUOĞLU ...........................111

Hasije DURAKU ............................ 81, 82, 130

Hassan AL-THANI......................................... 77

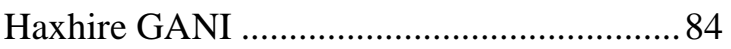

Hayato KURIHARA.......................................

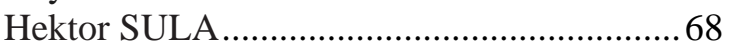

Helidon KLOSI..............................................126

Helidon NINO ................................................ 32

Henri KOLANI................................67, 84, 92

Herion DREDHA......................................... 102

Husham ABDELRAHMAN ..........................78

Hysni BENDO …...................................... 9,

\section{I}

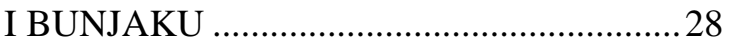

Ibrahim AVDI................................................. 32

Igor KAFTANDZIEV .............................94, 96

Ihor HAIDA ….................................................... 12

Ilda TAKA …......................................... 71, 72

Ilia MAZNIKU ……………..................... 71, 72

Ilir ALIMEHMETI .........................................63

Ilir HASANI ..........................38, 53, 70, 94, 96

Ilir HASMUCA …............................... 52, 113

Ilir PEPOSHI ........................................................ 51

Ilir SHABANI.................................................5 53

Ilir SHANI, ................................................91

Ilir SKENDULI …...........................33, 50, 51

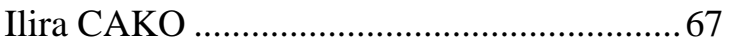

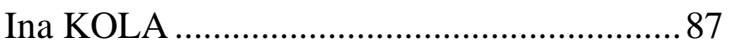

Ina UZUNI...................................................... 75

Indrit JAUPAJ ........................................... 19

Indrit TEMALI .............................................. 31

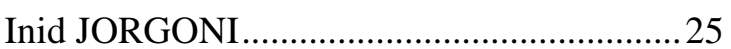

Ismael Yousif Mahmood ALOMAR ............. 78

Isuf BAJRAMI.................................................. 15

$J$

Jasmina KRÖPFL ................................13, 121

Jetmira KERXHALLIU ..................... 19, 21, 23

Jeton SHATRI ..............................56, 57, 137

Johana VRUHO ...........................................41

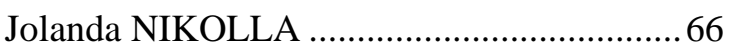

Jonela BURIMI................................................ 84

Jordan SAVESKI.............................................94

Jožeta POTRČA................................................. 26

Juxhin GJOSHE ............................................. 127

$\boldsymbol{K}$

K. HYSA …..........................................106,126

Kastriot HAXHIREXHA..... 10, 27, 62, 91, 94, 103, 105

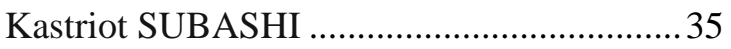

Katerina KASA........................................40, 43

Kenan BAYRAKCI ................. 37, 96, 113, 114 


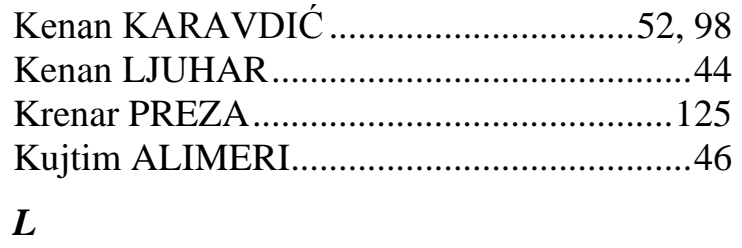

L. BYLYKBABSHI.......................................93

L. Laci ........................................................93

Lazar TODOROVIC ..............................55, 97

Leart BERDICA............................................107

Ledia QATIPI ..............................................107

Ledian FEZOLLARI................37, 96, 113, 114

Leodita GJANA ….........................................41

Leon KAZA ...............................................125

Lindita SEJFULLAHU-ELEZI....................110

Ljubica MIKJUNOVIKJ............................97

Lordian NUNCI ..........................................117

Lorena HAXHIHYSENI..............................42

Lorenc GJOKA ................................................34

Loreta AGOLLI ............................................83

Luan BAJRI …….........................................99

Luljeta STANAJ ............................................39

Lutfi LISHA ................................................32, 35

Lutfi ZYLBEARI...............................103, 105

Lutfi ZYLBEHARI.................................91, 94

\section{M}

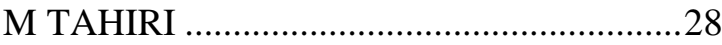

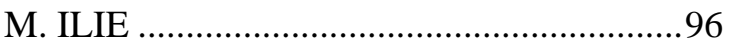

Majlinda NACO...............................................51

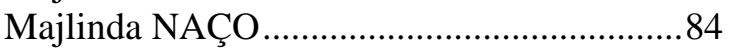

Majlindaj ZAHA ………………………...........128

Maksim BASHA …...........................................51

Manser ÇILI...................................................133

Manushaqe SARAÇI ............................39, 136

Mariola NDRECKA.........................................89

Marjan KAMILOSKI......................................55

Marjan.KAMILOSKI.....................................97

Marjeta BILAJ ..............................................86

Marjeta BILAJ(Miraka) ...................................85

Marko SPASOV ….......................................94

MarkusKONERT ...................................68, 71

Marsela SOPIQOTI .............................118, 120

Marsida KRASNIQI .......................40, 43, 138

Matilda IMERAJ........................................103

Mauro ZAGO ...................................................24

Maysa MOHAMED..........................44, 46, 47

Merita RUSTEMI-ELEZI ............................110

Milan SAMARDZISKI................................38

Muhamet PETROVA ......................................15

Mujo SUBAŠIĆ ..............................................44

Murat Onder DELIALIOGLU .....................114

MustafaXHANI............................................100

Myftar BARBULLUSHI ..............................108

Myriam HUNINK .........................................63
Myzafer KAÇI .........................................50, 93

$N$

Najada KALLASHI .........................................46

Najada KALLASHI (JAHIQI) .....................130

Nardi KOLA ...........................................87, 89

Natalija COKLESKA ..............................55, 97

Natasha MERKO ........64, 65, 81, 82, 130, 131

Neda TRAJKOVSKA....................................94

Nehat BAFTIU ….....................................28, 69

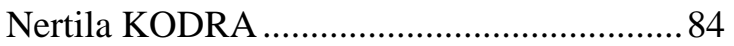

Nestor THERESKA ...................................... 108

Nevi PASKO ............................................. 108

Niketa KOLICI .................................... 106,126

Norik HOXHA...............................................15

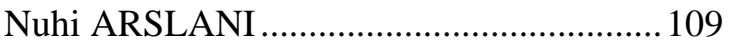

Nuhi ARSLLANI...........................................28

$\boldsymbol{O}$

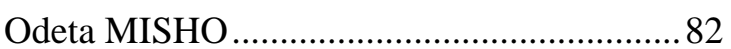

Oliver ARSOVSKI .........................................99

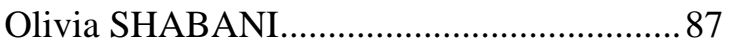

Onder M. DELIALIOGLU ............96, 113, 114

Onder Murat DELIALIOGLU........................37

Ormir SHURDHA ..............................................

$\boldsymbol{P}$

Parambath Arif NELLIYULLA.....................47

Petraq MUSTAQE................................34, 122

Petrika GJERGO_..................................117, 120

Petrit BIBERAJ …......................................137

Pirro PRIFTI ................................................... 58

Premtim RASHITI........................................28

Preveza ABRASHI ........................................14

Prof. Xheladin DRAÇINI ..............................134

$\boldsymbol{R}$

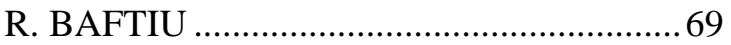

Raimonda TRAKO .....................................133

Ramona A. PARISI .....................................130

Rezart ÇIPI ..................................................115

Rezart XHANI ................................................. 100

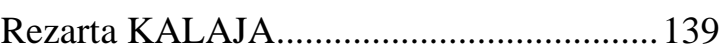

Rezarta KAPAJ.......................................41, 89

Rezeart DALIPI ........................38, 54, 94, 113

Ridvan ALIMEHMETI ....................19, 21, 23

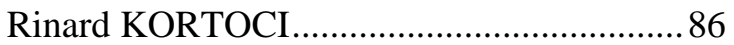

Risida GJONEJ .....................................79, 80

Rok KOLARIĆ ...........................................109

Roland KAZA.................................................. 86

Roland PERGJETANI ...................... 19, 21, 23

Roza LUCI............................................... 128

Rrustem MUSA …........................................... 12

Rudin DOMI .....................................69, 71, 100 
Rustem CELAMI ..10, 100, 101, 106,122, 123, 124, 125, 126

Ruzhdi REXHEPI 104

$S$

S. KRSTEVSKI .96

Sadi BEXHETI ............56, 57, 91, 94, 103, 105

Sadık Yıldırım Özgeçmiş.................................93

Sadri HULAJ ................................................29

Safet BEQIRI ................................................86

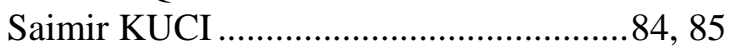

Sali GRADICA …........................................35

Sami KOCEKU ..........................................35, 61

Selda VEIZAJ ................................................

Selim Birol ..........................................................111

Selman DUMANI ............................................85

Serhii MITSUK..............................................12

Sevdije KOXHA ...........................................107

Shemsedin HASHANI ....................................12

Shpresa HASIMJA...........................................12

Sihana AHMETI-LIKA .................................105

Simon TRPESKI...........................................94

Simona KALŠEK................................. 26, 56

Skender BRATAJ .................10, 14, 16, 18, 19

Skender BUCI..................................26, 35, 61

Skender VELIU .....................................13, 121

Slavica LITAJKOVSKA ...............................55

Slavica SHUPERLISKA ...............................97

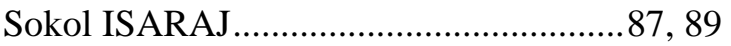

Sokol RUCI..............................................35, 61

Sokol XHEPA..............................117, 118, 120

Sokrat BERDUFI ........................................116

Sonila NIKA …..............................................128

Sonja BUTORAC (SARACI) .........................46

Sonja SARAÇI...............................................136

Sotiraq LAKO .................................................107

Stavri LLAZO ..................................84, 85, 118

Studim HYSA. ............................................129

Sulejman BAHA …................................71, 72

Suzana CAKONI ..........................................66

T

Teodor PEVEC

9,56
Teodora TODOROVA 38 Thoma KALEFI $19,21,23$

V

V S. REXHA .32

V. LEKA 106,126

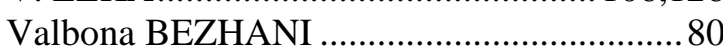

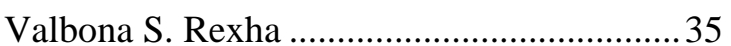

Valbona SELMANI .................................54, 55

Valentin VEJSELI ..........................................96

Vasilika PRIFTI............................................128

Vesel SKENDERI ............................................. 14

Veton ADEMI ......................................56, 137

Vidi DEMKO.....................................46, 132

Viktor MARKU ……...............................73, 98

Vilma CADRI...................................106, 108

Vilma MEMA ................................................ 138

Vilson RUCI .......................................... 79, 113

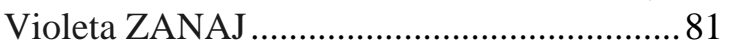

Vladimir FILAJ _..............................................90

Vrenos HODAJ.................................................. 59

W

Wojciech SZMIGIELSKI ............................46

X

Xheladin DRACINI ........................................ 10

XheladinELEZI.............................................91

Xhesika XHETANI ......................................132

$Y$

Ylber ZAMA

$54,55,113$

Yllka BARDHI 131

$Z$

Zamir DEMIRAJ ….......................................52

Zamira BEXHETI...............................103, 105

Zamira SHABANI .......................................134

Zef DELIA................................122, 125, 126

Zef PERDUKA............................................... 35

Zllato ALEKSOVSKI.............................55, 97 


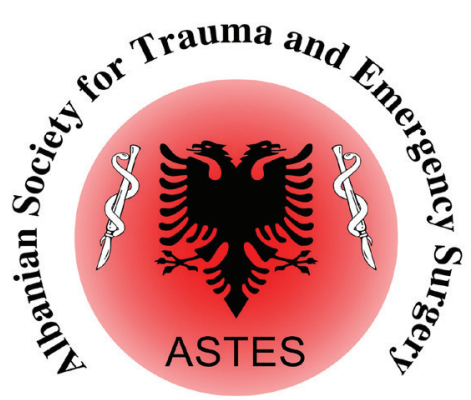

Organised by:

Albanian Society for Trauma and Emergency Surgery (ASTES)

www.astes.org.al

\title{
$4^{\text {th }}$ Annual Albanian Congress of Trauma and Emergency Surgery
}

\author{
November 13-14, 2020 \\ Tirana, Albania
}

\section{SAVE THE DATE!}

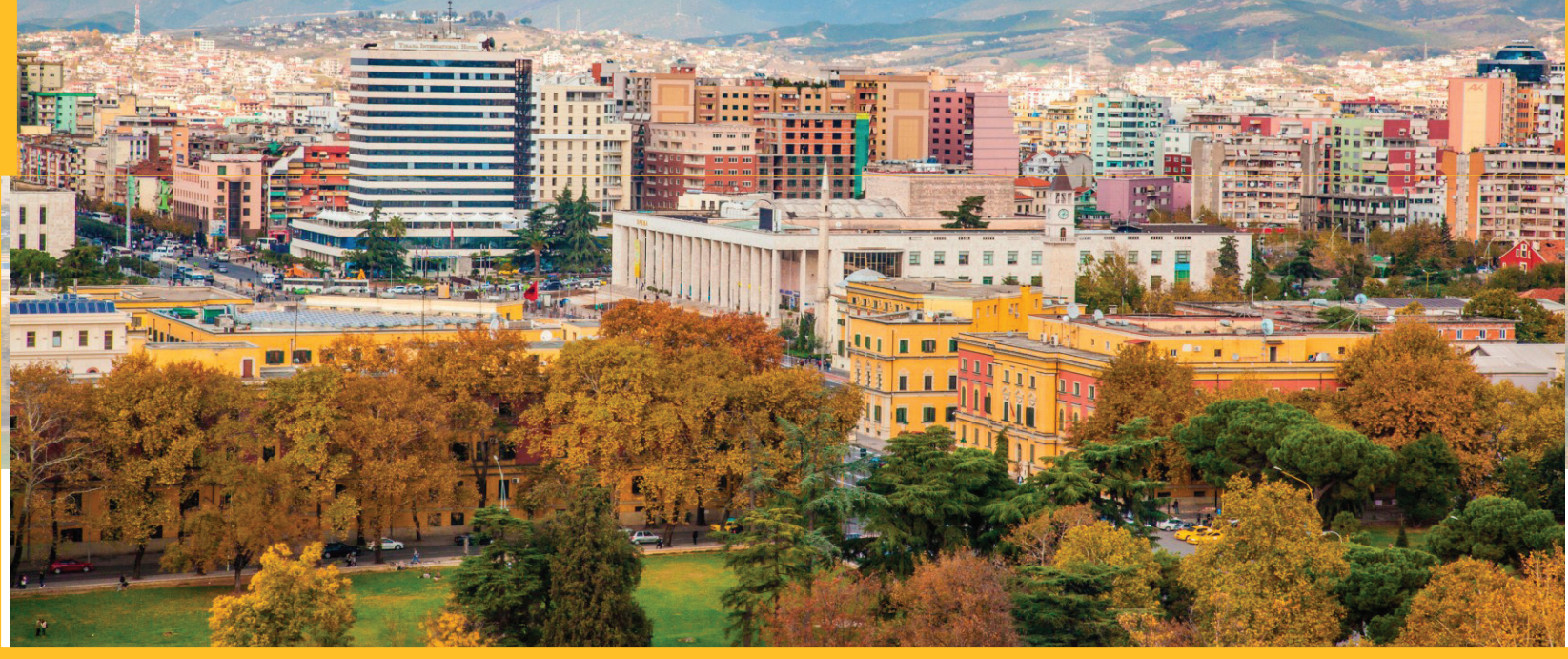

"Clear Vision, Careful Steps, ... Sure Success..." 